\title{
Using Machine Learning and Traditional Statistics to Explore Retention and Knowledge Structure in STEM with an emphasis on Physics
}

\author{
Cabot Alexander Zabriskie \\ West Virginia University, cazabriskie@mix.wvu.edu
}

Follow this and additional works at: https://researchrepository.wvu.edu/etd

Part of the Science and Mathematics Education Commons

\footnotetext{
Recommended Citation

Zabriskie, Cabot Alexander, "Using Machine Learning and Traditional Statistics to Explore Retention and Knowledge Structure in STEM with an emphasis on Physics" (2019). Graduate Theses, Dissertations, and Problem Reports. 4118.

https://researchrepository.wvu.edu/etd/4118

This Dissertation is protected by copyright and/or related rights. It has been brought to you by the The Research Repository @ WVU with permission from the rights-holder(s). You are free to use this Dissertation in any way that is permitted by the copyright and related rights legislation that applies to your use. For other uses you must obtain permission from the rights-holder(s) directly, unless additional rights are indicated by a Creative Commons license in the record and/ or on the work itself. This Dissertation has been accepted for inclusion in WVU Graduate Theses, Dissertations, and Problem Reports collection by an authorized administrator of The Research Repository @ WVU. For more information, please contact researchrepository@mail.wvu.edu.
} 


\title{
Using Machine Learning and Traditional Statistics to Explore Retention and Knowledge Structure in STEM with an emphasis on Physics
}

\author{
Cabot A. Zabriskie \\ Dissertation submitted \\ to the Eberly College of Arts and Sciences \\ at West Virginia University
}

in partial fulfillment of the requirements for the degree of

Doctor of Philosophy in

Physics

\author{
John Stewart, Ph.D., Chair \\ Gay Stewart, Ph.D. \\ Maura McLaughlin, Ph.D. \\ Karen Rambo-Hernandez, Ph.D. \\ Kathleen Koenig, Ph.D. \\ Department of Physics and Astronomy \\ Morgantown, West Virginia \\ 2019
}

Keywords: physics, education, conceptual inventories, retention, machine learning, item response theory

Copyright 2019 Cabot A. Zabriskie 


\title{
ABSTRACT \\ Using Machine Learning and Traditional Statistics to Explore Retention and Knowledge Structure in STEM with and emphasis on Physics
}

\author{
Cabot A. Zabriskie
}

\begin{abstract}
Retention of Science, Technology, Engineering, and Mathematics (STEM) students is a serious problem as STEM graduation rates continue to lag the growing demand for the skills taught by these degree programs. Critical to fixing this "leaky pipeline" is investment in improving retention in the first two years of college study and increasing and maintaining the interest of K-12 students in STEM. This thesis will address this in three parts. The first is through evaluation of conceptual tests used to evaluate course improvements to determine the structure student knowledge measured by them. The second part uses machine learning to construct early warning models of student failure in introductory physics courses to aid instructors in better targeting of interventions. The final part assesses the effectiveness of the Pulsar Search Collaboratory, an authentic science experience for middle and high school students, at encouraging K-12 students to pursue STEM degrees.
\end{abstract}




\section{ACKNOWLEDGMENTS}

First and foremost, I'd like to thank my thesis advisor, Dr. John Stewart. I have learned so much while working for you and I cannot imagine a better advisor and boss. The last few years have been transformative to me as a researcher and that would never have been possible without your help, guidance, and overall mentorship.

I would also like to thank my dissertation committee members, Dr. Gay Stewart, Dr. Maura McLaughlin, Dr. Karen Rambo-Hernandez, and Dr. Kathleen Koenig for taking time out of their busy schedules to be a part of this journey. Your questions have always been thoughtful and insightful and I appreciate everything that you have done.

Finally I would like to thank my friends and family, without your support I would never

have made it this far. I am truly grateful for all your sacrifice and for putting up with me throughout this whole crazy process. 


\section{Contents}

1 Introduction to Physics Education Research 1

1.1 Conceptual Knowledge of Physics . . . . . . . . . . . . . . . . . 3

1.1.1 The Force Concept Inventory _. . . . . . . . . . . . . . . . 4

1.1.2 The Conceptual Survey of Electricity and Magnetism . . . . . . . . 6

1.2 Reformed Instruction . . . . . . . . . . . . . . . . . . . . . 7

1.2 .1 Lecture-Based RBIS . . . . . . . . . . . . . . . . . . 9

1.2.2 Recitation or Discussion Based RBIS . . . . . . . . . . . . . 9

1.2 .3 Laboratory-Based RBIS . . . . . . . . . . . . . . . . . . . . . 10

1.2.4 Instructional Environment-Based RBIS . . . . . . . . . . . . . . 11

1.2 .5 Summary . . . . . . . . . . . . . . . . . . . . . . . 11

1.2.6 Adoption of Research-Based Materials . . . . . . . . . . . . . . 12

2 Statistical Methods $\quad 14$

2.1 Descriptive Statistics . . . . . . . . . . . . . . . . . . . 15

2.1.1 Measures of Central Tendency . . . . . . . . . . . . . . . . 15

2.1 .2 Variability . . . . . . . . . . . . . . . . . . . 16

2.2 Inferential Statistics $\ldots \ldots \ldots \ldots \ldots$

2.2 .1 Hypothesis Testing . . . . . . . . . . . . . . . . . . . . . 17

$2.2 .2 \quad$ Effect Size . . . . . . . . . . . . . . . . . . . . . . . . . . . . . . . . . . . . . 19

2.2 .3 Error . . . . . . . . . . . . . . . . . . . . . . . . . . 20

2.2 .4 Bootstrapping . . . . . . . . . . . . . . . . . . 20

\section{$\begin{array}{ll}\text { I Conceptual Survey Evaluation } & 21\end{array}$}

3 Evaluation of the Knowledge Structure Measured by the Force Concept Inventory 23

3.1 Factor Analysis and the FCI . . . . . . . . . . . . . . . . . 24

3.2 Item Response Theory and the FCI . . . . . . . . . . . . . . 26

3.3 The Structure of Knowledge . . . . . . . . . . . . . . . . . . . . . 28

3.4 Research Questions . . . . . . . . . . . . . . . . . . . . . . . . 30

3.5 Sample . . . . . . . . . . . . . . . . . . . . . . . . . . 32

3.6 Factor Analysis Using Item Response Theory . . . . . . . . . . . . . . . . . . 33

3.6 .1 Unidimensional Item Response Theory . . . . . . . . . . . . . . . . . 33

3.6 .2 Model Fit Statistics . . . . . . . . . . . . . . . . . . . . . . 33 
3.6.3 Multidimensional Item Response Theory . . . . . . . . . . . . . . . . 36

3.6.4 Exploratory and Confirmatory MIRT . . . . . . . . . . . . . . 37

3.6.5 Additional Analyses . . . . . . . . . . . . . . . . . . . . . 37

3.7 Results - Exploratory Analyses . . . . . . . . . . . . . . . . . 39

3.7.1 Exploratory Factor Analysis . . . . . . . . . . . . . . . . . . . 39

3.7.2 Correlation Analysis . . . . . . . . . . . . . . . . . . 43

3.7.3 A Theoretical Framework . . . . . . . . . . . . . . 46

3.7 .4 Reduced Exploratory Factor Analysis . . . . . . . . . . . . . . . . 50

3.8 Results - Confirmatory Analyses . . . . . . . . . . . . . . . . 54

3.8.1 Constrained MIRT . . . . . . . . . . . . . . . . . 55

3.8.2 Comparison with Original FCI Model . . . . . . . . . . . . . . . . . 62

3.9 Discussion . . . . . . . . . . . . . . . . . . . . . 63

3.10 Limitations . . . . . . . . . . . . . . . . . . . . . . . . . . . . . . . . . . . . . . . . . . .

3.11 Implications . . . . . . . . . . . . . . . . . . . . . . 70

3.12 Conclusions . . . . . . . . . . . . . . . . . . . . 71

4 Evaluation of the Knowledge Structure Measured by Conceptual Survey $\begin{array}{ll}\text { of Electricity and Magnetism } & 73\end{array}$

4.1 Reliability and Validity . . . . . . . . . . . . . . . . . . . . 74

4.2 Factor Analysis . . . . . . . . . . . . . . . . . . . . 76

4.3 Item Response Theory . . . . . . . . . . . . . . . . . . . . . . 77

4.4 Research Questions . . . . . . . . . . . . . . . . . . 77

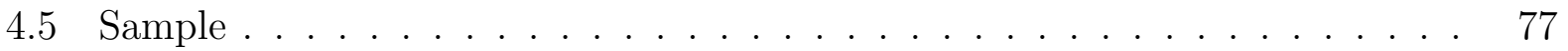

4.6 Exploratory Factor Analysis . . . . . . . . . . . . . . . . . . . . . . . . 80

4.6.1 Sample 1..................... 80

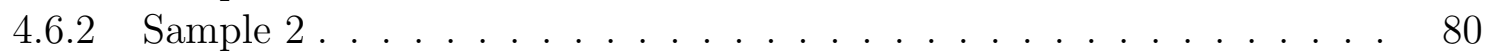

4.7 Theoretical Framework . . . . . . . . . . . . . . . . . . . . . 81

4.8 Model Transformation Plan . . . . . . . . . . . . . . . . . . . . . . 87

4.9 Results . . . . . . . . . . . . . . . . . . . . . . 9 90

4.9.1 Constrained MIRT . . . . . . . . . . . . . . . . . . . . . . . . . . . . . . . . 90

4.9.2 Analysis of Optimal Models . . . . . . . . . . . . . . . . . 94

4.9.3 Correlation of Abilities . . . . . . . . . . . . . . . . . . . . 97

4.9 .4 Analysis of Reserved Items . . . . . . . . . . . . . . . . . . . . . . . . . . . . . . . . . . . . .

4.10 Discussion . . . . . . . . . . . . . . . . . . . . . . 101

4.11 Implications . . . . . . . . . . . . . . . . . . . . . . 106

4.12 Limitations . . . . . . . . . . . . . . . . . . . . . . . . . . . . . . . . . . . . . . . . . . . . . . . . . . . . . .

4.13 Conclusion . . . . . . . . . . . . . . . . . . . . . . . . . . . . . . . . . . . . . . . . . . .

4.14 MIRT Analysis Summary . . . . . . . . . . . . . . . . . . . 109

\section{Using Machine Learning to Predict Course Grade 111}

$5 \quad$ Using Machine Learning to Predict Course Grade 113

5.1 Educational Data Mining . . . . . . . . . . . . . . . . . . 115

5.2 EDM and Grade Prediction . . . . . . . . . . . . . . . . . 117 
5.3 Research Questions . . . . . . . . . . . . . . . . . . . . . . . . . . . 119

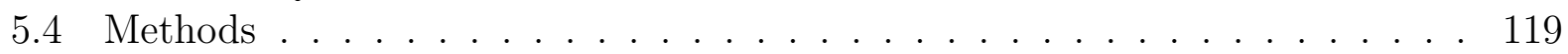

5.4 .1 Context for Research . . . . . . . . . . . . . . . . . . . . . . . . . 119

5.4 .2 Sample . . . . . . . . . . . . . . . . . . . . . . . . . . . . 121

5.4 .3 Variables . . . . . . . . . . . . . . . . . . . . . . . . . . . . 121

5.4 .4 Classification Models . . . . . . . . . . . . . . . . . . . . . . . 123

5.4.5 Classification Methods . . . . . . . . . . . . . . . . . . . . . 127

5.4.6 Opening the "Black Box" of Machine Learning: Local Interpretable Model-Agnostic Explanations . . . . . . . . . . . . . . . . 131

5.5 Results . . . . . . . . . . . . . . . . . . . . . . . . . . . . 132

5.5.1 Physics 1 Model Performance . . . . . . . . . . . . . . . . . . . 132

5.5 .2 Physics 1 Variable Importance . . . . . . . . . . . . . . . . . . . . 135

5.5.3 Physics 2 Model Performance and Variable Importance . . . . . . . . 144

5.5 .4 LIME Results . . . . . . . . . . . . . . . . . . . . . . . . . . . . . . 152

5.6 Discussion . . . . . . . . . . . . . . . . . . . . . . . . 153

5.6 .1 Additional Observations . . . . . . . . . . . . . . . . 157

5.6 .2 Recommendations . . . . . . . . . . . . . . . . . . . . . . . 158

5.6 .3 Limitations . . . . . . . . . . . . . . . . . . . . . . . 158

5.7 Conclusions . . . . . . . . . . . . . . . . . . . . . . . . . . . . . . . . . 159

III The Pulsar Search Collaboratory 160

6 Introduction to Pulsar Search Collaboratory 161

6.1 Introduction . . . . . . . . . . . . . . . . . . . . . . . . 162

6.1 .1 The Original PSC . . . . . . . . . . . . . . . . . . . . . . . . 162

6.1 .2 The New PSC . . . . . . . . . . . . . . . . . . . . . . . . 164

7 Programmatic Evaluation of the New PSC $\quad 167$

7.1 Student Engagement . . . . . . . . . . . . . . . . . . . . . . . 168

7.2 School Participation . . . . . . . . . . . . . . . . . . . . . . 169

7.3 Capstone Qualification . . . . . . . . . . . . . . . . . . . 172

7.4 The Qualification Tests . . . . . . . . . . . . . . . . . 178

8 Evaluation of the New PSC's Effect on Student STEM Intentions 184

8.1 Student Analysis . . . . . . . . . . . . . . . . . . . . . . . . . . 189

8.1 .1 Pre-Survey Results . . . . . . . . . . . . . . . . . . . . . . . . . . 189

8.1 .2 Post-Survey Results . . . . . . . . . . . . . . . . . . . . . . . . . . . . 190

8.1 .3 Matched Samples . . . . . . . . . . . . . . . . . . . . . . . . . . 190

8.2 Conclusions . . . . . . . . . . . . . . . . . . . . . . . . . . . 191

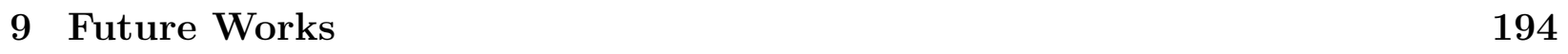

$\begin{array}{ll}10 \text { Additional Work } & 197\end{array}$

$\begin{array}{ll}\text { Bibliography } & 202\end{array}$ 


\section{List of Tables}

3.1 Exploratory Factor Loadings of the FCI . . . . . . . . . . . . . . . . 41

3.2 EFA Fit Statistics by Number of Factors FCI . . . . . . . . . . . . . . . 43

3.3 Theoretical model of Newtonian mechanics as tested by the FCI . . . . . . . 49

3.4 EFA for the reduced FCI (varimax rotation) . . . . . . . . . . . . . . 52

3.5 FCI Model Transformations . . . . . . . . . . . . . . . . . . 55

3.6 Optimal FCI Model Principles . . . . . . . . . . . . . . . . . . . . . 59

3.7 Optimal FCI Model . . . . . . . . . . . . . . . . . 61

4.1 EFA CSEM Sample 1 . . . . . . . . . . . . . . . . . . . . . . 81

4.2 CSEM Sample 1 Optimal EFA Model . . . . . . . . . . . . . . . . . 82

4.3 EFA CSEM Sample 2 . . . . . . . . . . . . . . . . . . . . . 83

4.4 CSEM Sample 2 Optimal EFA Model . . . . . . . . . . . . . . . . . 84

4.5 Theoretical model of electricity and magnetism as tested by the CSEM . . . 88

4.6 CSEM Sample 1 Model Transformations . . . . . . . . . . . . . . . 91

4.7 CSEM Sample 2 Model Transformations . . . . . . . . . . . . . . . . . . . . 91

4.8 Optimal CSEM MIRT Models for Samples 1 and 2 . . . . . . . . . . . . . 95

4.9 Parameter Differences Between Sample 1 and Sample 2 CSEM MIRT Models 97

4.10 CSEM Reserved Item Comparisons . . . . . . . . . . . . . . . . . . . 99

5.1 List of Modeled Variables . . . . . . . . . . . . . . . . . . . . . 122

5.2 Confusion Matrix Example . . . . . . . . . . . . . . . . . . . . 124

5.3 Physics 1: Model Performance . . . . . . . . . . . . . . . . . 134

5.4 Physics 1: Optimal institutional logistic model. The z-score is the test statistic used to determine if the odds ratio is statistically significant. . . . . . . . . 136

5.5 Physics 1: Logistic models in-class variables only. . . . . . . . . . . . . 139

5.6 Physics 1: Logistic models combining in-class and institutional variables. . . 140

5.7 Physics 2: Model Performance . . . . . . . . . . . . . . . . . 145

5.8 Physics 2: Optimal institutional logistic model. . . . . . . . . . . . . . 146

5.9 Physics 2: Logistic models in-class variables only. . . . . . . . . . . . . . 148

5.10 Physics 2: Logistic models in-class and institutional variables only. . . . . . 150

7.1 Passage of Subtests per Year . . . . . . . . . . . . . . . . 183

8.1 Pre-Survey Results . . . . . . . . . . . . . . . . . . . . . 190

8.2 Post-Survey Results . . . . . . . . . . . . . . . . . . . . . . . . . . 190

8.3 Matched PSC Participants Pre/Post Survey Results . . . . . . . . . . . . . . 191 
8.4 Matched Control Participants Pre/Post Survey Results . . . . . . . . . . . . 191 


\section{List of Figures}

1.1 Gain vs. Pretest for Traditional Instruction and Interactive Engagement . . 8

1.2 Effect Size of Reformed Instruction By Discipline . . . . . . . . . . . . . . . 12

3.1 FCI Scree Plot . . . . . . . . . . . . . . . . . . . . . . . . . . . . . . . . . . .

3.2 Correlation matrix for all FCI items . . . . . . . . . . . . . . . . . . . 44

3.3 Partial correlation matrix for all FCI problems correcting for total FCI score 45

3.4 Venn Diagram of the distribution of problems in the FCI . . . . . . . . . . 53

3.5 Correlation Matrix of Student Ability in Optimal Model . . . . . . . . . . . 62

4.1 Correlation matrix of ability scores for CSEM Sample 1 . . . . . . . . . . 98

4.2 Correlation matrix of ability scores for CSEM Sample 2 . . . . . . . . . . . 100

5.1 ROC Curve Example with 95\% CI. The x-axis represents the false positive rate with specificity defined as the true negative rate, $T N /(T N+F P)$. The $\mathrm{y}$-axis represents the true positive rate or sensitivity, $T P /(T P+F N)$. AUC is the area under the ROC curve and is analogous to accuracy. A ROC curve for a perfectly fit model will form a right angle at the upper left corner and have and AUC of 1.0 while a model that is no better than guessing will be collinear with the line in the middle of the plot and have an AUC of 0.50. . . 126

5.2 Physics 1: Weekly Model Performance . . . . . . . . . . . . . . . 133

5.3 Physics 1: Institutional Variable Importance . . . . . . . . . . . . . . . . 137

5.4 Physics 1: Weekly Variable Importance . . . . . . . . . . . . . . . . . . . . . 142

5.5 Physics 1: Optimal Model Confusion Matrices . . . . . . . . . . . . . . . . 143

5.6 Physics 2: Institutional variable importance . . . . . . . . . . . . . . . 147

5.7 Physics 2: Weekly variable importance . . . . . . . . . . . . . . . . 149

5.8 Physics 2: Optimal Model Confusion Matrices . . . . . . . . . . . . . . . . 151

5.9 Physics 1: Example LIME result . . . . . . . . . . . . . . . . . . 153

7.1 Participating Schools Based on Active Members . . . . . . . . . . . . . . . . 169

7.2 Participating Schools Based on New Members . . . . . . . . . . . . . . . 170

7.3 Participating Schools Based on Active New Members . . . . . . . . . . . . . 171

7.4 Total Participating Members By Year and Capstone Qualification . . . . . . 173

7.5 Pulsar Candidate Pointings Completed by Students Who Qualified for Capstone in the Original PSC - Lower 95\% of Plot Scorers . . . . . . . . . . . . . . . 174

7.6 Pulsar Candidate Pointings Completed by Students Who Qualified for Capstone in the New PSC - Lower 95\% of Plot Scorers . . . . . . . . . . . . . . 175 
7.7 Pulsar Candidate Pointings Completed by Students Did Not Qualify for Capstone in the Original PSC - Lower 95\% of Plot Scorers . . . . . . . . . . . . 176

7.8 Pulsar Candidate Pointings Completed by Students Who Did Not Qualify for Capstone in the New PSC - Lower 95\% of Plot Scorers . . . . . . . . . . 177

7.9 Pulsar Candidate Pointings Completed by Students Who Qualified for the PSC and for Capstone by Year - Lower 95\% of Plot Scorers . . . . . . . . 178

7.10 Pulsar Candidate Pointings Completed by Students Who Did Not Qualify for the PSC and for Capstone by Year - Lower 95\% of Plot Scorers . . . . . . . 179

7.11 Total Participating Members By Year and Number of Sub-Tests Passed . . . 180

7.12 Cumulative Density Plots of Original PSC Subtest Attempts . . . . . . . . . 181

7.13 Cumulative Density Plots of New PSC Subtest Attempts . . . . . . . . . . 182

8.1 PSC Post Survey Programmatic Responses Part 1 . . . . . . . . . . . . . . 186

8.2 PSC Post Survey Programmatic Responses Part 2 . . . . . . . . . . . . . 187

8.3 PSC Post Survey Programmatic Responses Part $3 \ldots \ldots$. . . . . . . . . . 188 


\section{Chapter 1}

\section{Introduction to Physics Education Research}


Workforce demand for Science, Technology, Engineering, and Mathematics (STEM) graduates has grown significantly over the past decade, with the number of jobs requiring at least a STEM bachelor's degree growing to comprise around $20 \%$ of the workforce [1]. This growth in the STEM job sector has put significant pressure on universities to increase the number of students who graduate with STEM degrees. In their 2012 report, the President's Council of Advisors on Science and Technology emphasized the need to improve retention of STEM students to avoid a projected 1 million STEM job candidate shortfall over the next decade [2]. Improving the STEM career pipeline is crucial to future economic growth and a multifaceted solution involving improvements at all levels is necessary to overcome these shortfalls.

The research contained herein will focus on first addressing areas where the STEM pipeline can and must be improved as well as developing the tools necessary to empower educators to improve the STEM pipeline. This will be subdivided into three parts. These parts represent separate approaches to addressing the issues related to STEM retention and together provide multiple avenues toward decreasing STEM attrition rates. Each will be discussed in order.

Part I introduces a new factor analysis technique to probe for the first time the knowledge structure of two widely used instruments for measuring conceptual knowledge [3]: the Force Concept Inventory (FCI) and the Conceptual Survey of Electricity and Magnetism (CSEM). This analysis will support the need to develop modern instruments for understanding student conceptual physics knowledge. These techniques will also be central to the construction of the next generation of conceptual instruments in physics. At the college level, we developed a machine learning model of student success in introductory physics 
courses, detailed in Part II, which can allow instructors to understand factors influencing success in the course well before the first in-semester test. Because the decision to pursue a STEM degree often occurs before a student reaches college [4-6], Part III evaluates a pre-college program that can help to influence this decision. Engaging students in authentic STEM experiences early allows them to begin to develop an identity as a scientist and provides them a clearer picture of what a career in STEM entails. The Pulsar Search Collaboratory is evaluated to determine how well it serves to meet these goals.

\subsection{Conceptual Knowledge of Physics}

The first attempts to study student conceptual knowledge in physics began in the late 1970s when education researchers began investigating how students develop conceptual understanding of scientific concepts [7, 8]. In 1980, Champagne, Klopfer, and Anderson developed the Demonstration, Observation, and Explanation of Motion test (D.O.E.) to evaluate student preconceptions of motion [9]. Prior to this study, most work on understanding success in physics courses examined logical and mathematical skills, while ignoring physics conceptual understanding. In order to determine if conceptual understanding was important beyond logical reasoning and mathematics skills, Champagne et al. also administered a logical reasoning test and a mathematical skills test to the students and were able to show that all three test results were highly correlated to student success in an introductory classical mechanics course [9]. This study showed, for the first time, that there were multiple facets to student conceptual understanding of physics, including prior physics conceptions. Two years later, in 1982, Clement showed that students have alternate views of the relationship between 
force and motion, such as the common misconception that "motion implies force" [10], that affect student understanding of physics. This study, as well as a study by McDermott in 1984, showed the importance of qualitative understanding and the need to explicitly address student difficulty with conceptual understanding in physics courses [11]. In 1985, Halloun and Hestenes introduced their first mechanics diagnostic test explicitly developed to explore student conceptual understanding of physics [12]; this test would serve as the progenitor of the conceptual instruments in use today.

\subsubsection{The Force Concept Inventory}

The Force Concept Inventory (FCI) was introduced 25 years ago and has become one of the most used and most studied instruments in Physics Education Research (PER) [13]. Measurements using the instrument have been important in the recognition that traditional instruction was not sufficient for students to develop a conceptual understanding of Newton's laws [14]. Its success was followed by the development of numerous other conceptual instruments, some of which found wide-spread use, including the Force and Motion Conceptual Evaluation (FMCE) [15], the Conceptual Survey of Electricity and Magnetism (CSEM) [16], and the Brief Electricity and Magnetism Assessment (BEMA) [17].

As an instrument, the FCI is a 30-item multiple-choice test that includes conceptual questions about Newton's laws, kinematics, and forces [13]. Each item has five possible responses with many representing "commonsense alternative" responses developed to include common misconceptions. These misconceptions include, but are not limited to: the belief in "impetus" or the motive force that must be supplied and can run out, that motion implies an 
active force, and heavier objects fall faster. Structurally, the FCI contains some individual items and some items that are grouped into blocks which share a common stem. Though some items share a common stem, not all probe the same concept within that stem. The FCI has repeatedly been shown to be an instrument with high internal consistency as measured by Cronbach's alpha $[18,19]$. Cronbach's alpha measures the degree to which questions which measure the same concept are correlated with each other [20].

As designed, the FCI is not intended to provide granular understanding of student knowledge, but instead to probe the overall Newtonian-like thinking of the students, i.e. whether they have a "Newtonian force concept." With this in mind, the authors of the FCI recommended the instrument for three major applications. The first application was for the uncovering of misconceptions, where students could be interviewed about their answers on the FCI to uncover the reasoning behind the selection of the aforementioned "commonsense alternatives." The second use is as an evaluation of instruction. This is the application most familiar to physics instructors who commonly calculate the normalized gains (a measure of how much students learned which accounts for how much they could have learned, see Eqn 1.1) [14] between FCI pre- and post-instruction scores to determine how much their students have learned in their course. The final recommended use was as a placement exam. The authors of the FCI cautioned against using the FCI alone for this purpose or as means to select between regular and honors physics courses at the high school level [13]. Since its introduction, the FCI has been revised and its current form was published with Mazur's Peer Instruction [21] and is available at PhysPort [22]. 


\subsubsection{The Conceptual Survey of Electricity and Magnetism}

The Conceptual Survey of Electricity and Magnetism (CSEM) is a 32-question conceptual instrument designed to measure student understanding of electricity and magnetism. The CSEM was introduced nearly 20 years ago and has become one of the most used tools for understanding conceptual knowledge of electricity and magnetism [16]. The CSEM was developed following the success of the Force Concept Inventory (FCI) [13] in demonstrating the ineffectiveness of traditional instruction in fostering conceptual learning [14]. Like the FCI, the CSEM was developed to test student misconceptions as well as their physics knowledge. This instrument covers concepts often found in introductory electricity and magnetism courses such as the Coulomb force law, electric and magnetic fields, induction, and electric potential [16]. The CSEM was originally developed by Maloney et al. by combining the items from two prior surveys from Hiegeelke and O'Kuma. These earlier surveys probed the understanding of electricity and magnetism separately [23]. These two surveys were combined after many iterations producing the current version of the CSEM which can be found at PhysPort [22]. A complete list of the concepts the CSEM was designed to measure can be found in Maloney et al. [16].

The other instrument commonly used to measure student conceptual learning in electricity and magnetism is the 30-item Brief Evaluation of Electricity and Magnetism (BEMA) [17]. Pollock found the CSEM and BEMA to be equally effective for evaluating conceptual learning with slight variations in the content covered by each instrument [24]. The BEMA will not be discussed in the research presented here. 


\section{Summary}

These instruments have been used to help understand the effect of pedagogical innovations, the challenges of learning physics, and, though not discussed in detail here, issues of inclusion in physics. The impact of these instruments has been immense and they have been used in a substantial subset of the studies performed in PER [3].

\subsection{Reformed Instruction}

In his 1998 study [14], Hake examined the FCI scores from 62 separate institutions comparing the effectiveness of reformed instruction. To compare institutions with different student populations, Hake used the normalized gain, Eqn 1.1,

$$
\langle g\rangle=\frac{\left\langle S_{f}\right\rangle-\left\langle S_{i}\right\rangle}{100-\left\langle S_{i}\right\rangle}
$$

where $\left\langle S_{i}\right\rangle$ is the class pretest average and $\left\langle S_{f}\right\rangle$ is the class post-test average on a scale of 0 -100. Hake classified high- $g$ as $\langle g\rangle \geq 0.7$, medium- $g$ as $0.7>\langle g\rangle \geq 0.3$, and low- $g$ as $\langle g\rangle<0.3$. Figure 1.1 presents a plot of the average gain $\langle$ gain $\rangle=\left\langle S_{f}\right\rangle-\left\langle S_{i}\right\rangle$ against pretest percentage score for the 62 institutions studied by Hake. As can be seen in Fig. 1.1, those institutions utilizing reformed instruction have improved normalized gains over those institutions which do not. In this figure, the lines represent the normalized gain thresholds with steeper lines representing greater gains. By weighting the gains on the FCI against the initial performance, it is possible to compare gains across institutions. This study catalyzed the push to adopt reformed instructional techniques (sometimes refered to as research-based instructional strategies or RBIS) in the physics classroom. 


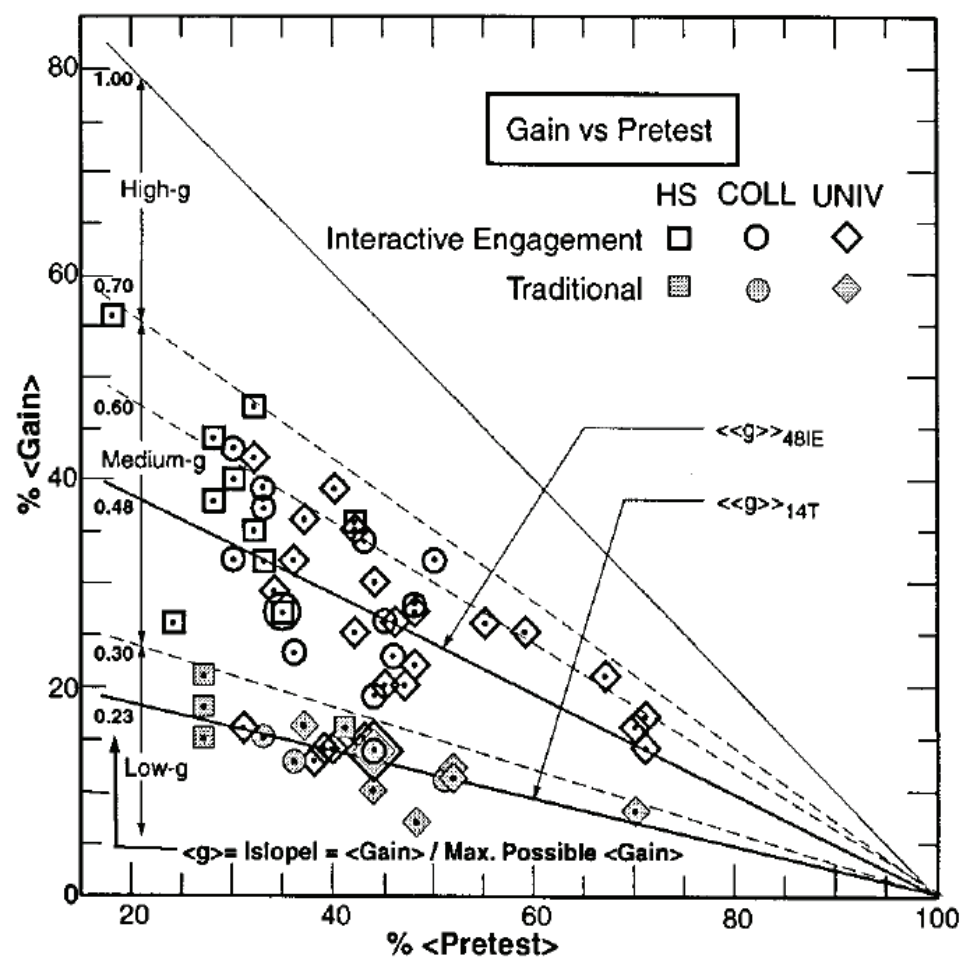

Figure 1.1: Gain vs. pretest for traditional instruction and interactive engagement [14]. Each $\langle g\rangle$ line represents the threshold for normalized gain given an average pre-test score. There were 48 interactive engagement (IE) samples and 14 traditional instruction (T) samples in the study. HS = high school, COLL $=$ college, UNIV $=$ university.

Since Hake's first study of reformed instruction in 1998, there has been a flurry of activity on developing, validating, and analyzing reformed instructional techniques in the physics classroom. In their 2014 review of PER, Docktor and Mestre [3] classified modern reformed physics instruction into five broad categories: lecture-based, recitation or discussion methods, laboratory methods, structural changes to the classroom environment, and general instructional strategies and materials. In the following subsections, examples in each of the first four categories will be reviewed and, where applicable, those implemented in the instructional environments studied will be discussed. The final category of "general instructional strategies and materials" is too broad to be summarized in this discussion. 


\subsubsection{Lecture-Based RBIS}

Lecture-based reformed instruction is designed to evolve the traditional lecture environment from a passive experience for the students into an active one [25]. One such way of improving student lecture engagement is through the use of "clickers" [3, 26, 27], which were used in each of the samples evaluated in this thesis. This method utilizes electronic voting machines, "clickers," to allow students to select multiple-choice answers from a problem posed to the entire class. This methodology not only allows students to be actively engaged in the lecture, but provides immediate feedback to instructors regarding student understanding of concepts being taught. One varient of this method, Peer Instruction, was popularized by Mazur [21]. In Peer Instruction, students discuss questions in small groups after providing their individual answers with the clickers. In a later 2007 study of data from 30 courses across 11 colleges and universities, Crouch, Watkins, Fagen, and Mazur [28] found the implementation of Peer Instruction led to much higher normalized gains than were found in traditionally taught courses.

\subsubsection{Recitation or Discussion Based RBIS}

The Tutorials in Introductory Physics [29] from the University of Washington, developed from the understanding gained in two previous studies [30, 31], are recitation or discussion methods and were used in some of the samples studied herein. The tutorial methodology uses a combination of workbooks (involving pretests, worksheets, and homework) designed to guide students toward conceptual understanding and trained learning or graduate assistants who work with students in a low-stakes environment (in our case the first half of the lab 
section). The impetus behind the tutorials was the historic focus of physics courses on student ability to solve quantitative problems and assume that qualitative understanding would be developed naturally. As shown by McDermott and Shaffer [30], students do not naturally develop strong qualitative understanding and therefore the tutorial methodology was created to walk students through the process of understanding by confronting their misconceptions and forcing students to think critically about what is physically happening. Finkelstein and Pollock [32] found that the use of tutorials helped to improve student conceptual understanding as well as course outcomes, but cautioned that the tutorials must be fully integrated into the course structure to be effective.

\subsubsection{Laboratory-Based RBIS}

An early synthesis by Alan Van Heuvelen [33] highlighted the problems with traditional labs where students would often leave knowing little more than when they entered. These environments focused on "cookie-cutter" experiments and taught students facts instead of processes. One method of rectifying this is the use of Investigative Science Learning Environment (ISLE) labs. An ISLE lab requires the student to design their own experiment and solve a research problem themselves rather than follow a pre-built lab [34]. ISLEtype labs have been shown to improve student scientist-like thinking and improve the skills associated with being a scientist such as experimental design and data analysis [3]. However, recent research has called into question the overall effectiveness of lab-based instruction $[35,36]$. In a multi-institution, multi-course study, Holmes, Olsen, Thomas, and Wieman [36] found no discernible differences on final exam scores between students who took the lab and those who did not. This study evaluated multiple populations and instructional 
techniques and calls into question whether the laboratory environment is the correct domain in which to focus interventions designed to affect course outcomes.

\subsubsection{Instructional Environment-Based RBIS}

Modifications to the learning environment itself have also been implemented with success. Methods such as the student-centered active learning environment for undergraduate programs (SCALE-UP) [37] rearrange the lecture environment by placing students in small work groups. Each group has access to a laptop, whiteboard, and each other for hands-on activities that occur in conjunction with the lecture. This methodology combines the lab and lecture into a single cohesive experience and has been shown to improve student outcomes in introductory courses [3].

\subsubsection{Summary}

The broader effects of reformed instruction on science education were synthesized in 2014 by Freeman et al. [38]. Their meta-analysis of 225 other studies found the use of reformed instruction to be an effective means of decreasing failure rates as well as increasing learning in STEM courses across multiple disciplines as shown in Fig 1.2.

Hedges's $g$ is similar to Cohen's $d$ and measures the improvement of some learning outcome, such as course average or post-test score. The numbers under each point represent the number of studies included in each analysis. 

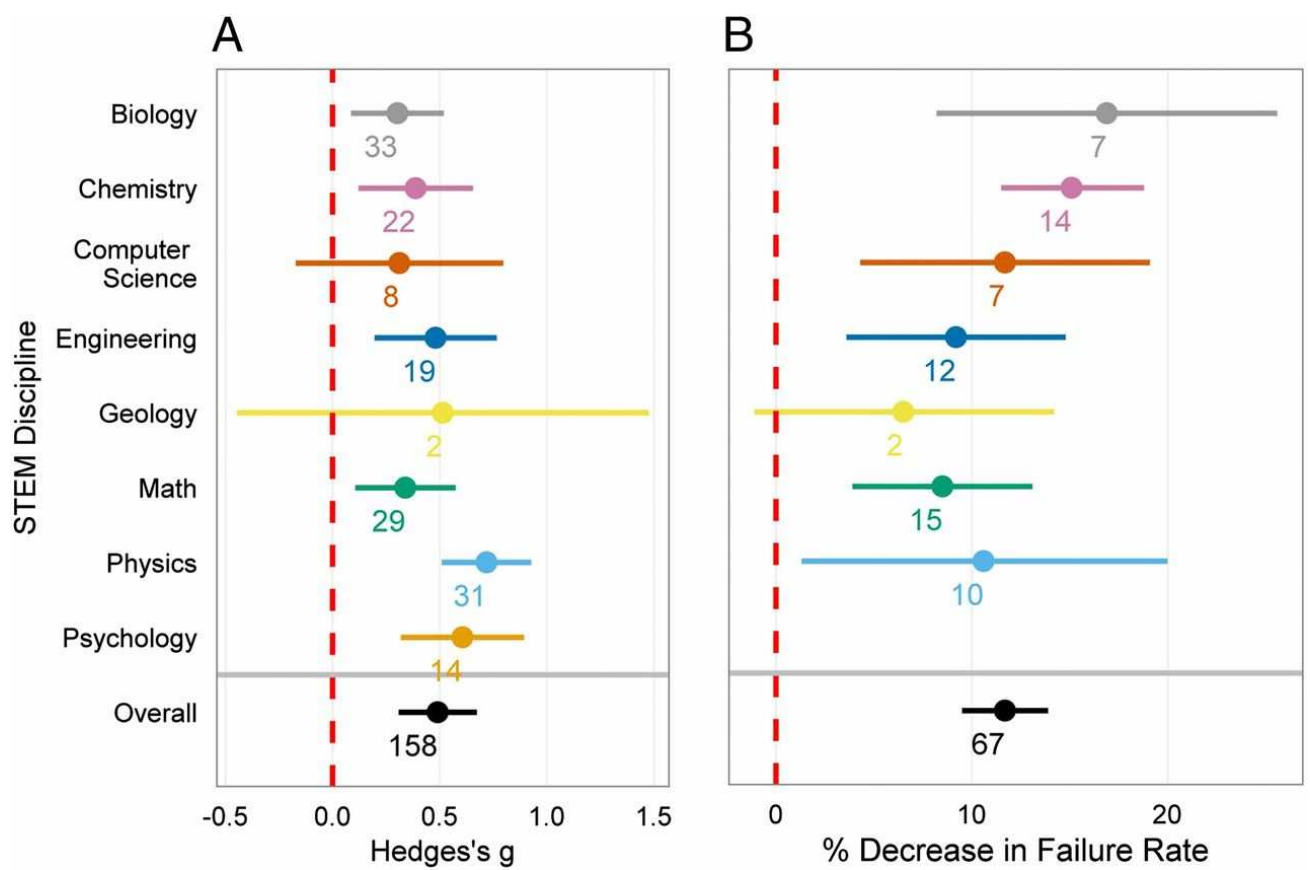

Figure 1.2: Effect Size of Reformed Instruction By Discipline [38].

\subsubsection{Adoption of Research-Based Materials}

Despite the wide number of techniques available to physics instructors and the quantifiable gains from implementing reformed instruction, the use of these techniques has not been adopted in most physics programs [39]. In their survey of 722 physics faculty, Henderson et al. found that while $87 \%$ of those surveyed were familiar with RBIS, less than half were currently implementing them. Beyond that, of those who had attempted to use RBIS, a third discontinued using RBIS.

Henderson et al. proposed that the abandonment of RBIS comes from multiple vectors. When introduced to faculty during workshops, talks, etc. RBIS are often oversold and the difficulties associated with their implementations not fully articulated. This can lead to faculty being surprised at not seeing immediate improvement and being unprepared for student resistance to these new methodologies. Additionally, they identify a lack of support 
for faculty during the implementation phase, where faculty do not have experts or other practitioners to consult with as problems arise. Overall this illustrates that the primary issue with the adoption of RBIS in physics is situated not in dissemination of the techniques, but in providing the support necessary to continue their use. 


\title{
Chapter 2
}

\author{
Statistical Methods
}


Statistics represent a way of mathematically understanding large sets of data and is divided into two major categories: descriptive and inferential statistics. The value of statistics lies in its capacity to make trustworthy (within a certain confidence interval) statements on a population based on a randomly drawn sample from that population. Sample and population have distinct definitions with population referring to all members of a group that is being studied and sample referring to a representative subset of that population. When performing statistical analyses, it will almost always be the sample statistic that is being calculated as it is often impossible to collect data from every single member of a population. If a sample is large and selected randomly, it can serve as a representation of the total population and the sample statistic can be thought of as a good approximation of the population statistic. This work uses multiple statistical techniques. This chapter summarizes the statistical methods used throughout. Additional methods will be introduced as needed.

\subsection{Descriptive Statistics}

Descriptive statistics are often the first step in any statistical analysis and provide the researcher with a overview of the general properties of the sample. In this section, two of the most important descriptive measures are discussed: central tendency and variability.

\subsubsection{Measures of Central Tendency}

Measures of central tendency are used to characterize a distribution by defining the most "typical" value for a sample or population. The three measures of central tendency are mean, median, and mode. The mean (arithmetic average) was the only measure used in this thesis. The population mean $\mu$ is defined in Eqn 2.1 where the sum is taken over all 
individuals, $i$, in the population. The definition for the sample mean, Eqn 2.2, follows the same mathematical form; however, the sum is taken over all individuals in the sample.

$$
\begin{gathered}
\mu=\frac{\sum_{i} X_{i}}{N} \\
\bar{X}=\frac{\sum_{i} X_{i}}{n}
\end{gathered}
$$

where $N$ is the size of the population, $n$ is the size of the sample, and $X_{i}$ is a measure of individual $i$. The mean is directly related to the variance and standard deviation and is often the best choice for a measure of central tendency. However, for skewed or otherwise non-symmetrically distributed data, other measures of central tendency may be more useful [40].

\subsubsection{Variability}

Variability describes the spread of data in a distribution around the mean value using variance and the square root of the variance, the standard deviation.

$$
\begin{aligned}
& \sigma^{2}=\frac{\sum_{i}\left(X_{i}-\mu^{2}\right)^{2}}{N} \\
& s^{2}=\frac{\sum_{i}\left(X_{i}-\bar{X}^{2}\right)^{2}}{n-1}
\end{aligned}
$$

The population variance, $\sigma^{2}$, is defined in Eqn. 2.3 and the sample variance, $s^{2}$, is defined in Eqn. 2.4, where the sum is taken over all individuals $i$ in the population in Eqn 2.3 and 
all individuals in the sample in Eqn. 2.4. The sample variance differs from the population variance by a factor of $n-1$ in the denominator; this change allows the sample variance to serve as an unbiased estimate of the population variance $\sigma^{2}$ because smaller samples will tend toward smaller variability. The variance will be zero in cases where all of the data fall at the mean. Because the variance is squared, the units of the variance are also squared, making it difficult to compare to the original data and mean. For this reason, the square root of the variance, called the standard deviation, is often reported.

In cases where multiple samples have been taken and separately analyzed it is more useful to report the standard error of the mean (standard error) as shown in Eqn. 2.5. The standard error, $S E$, is the standard deviation of mean scores around the grand mean of all of the samples [41]

$$
S E=\frac{\sigma}{\sqrt{N_{s}}}
$$

where $N_{s}$ is the total number of separate samples. In this case the data itself does not need to be normally distributed. Instead only the means from the samples drawn from the population will be normally distributed about the true mean.

\subsection{Inferential Statistics}

\subsubsection{Hypothesis Testing}

Hypothesis testing, also known as significance testing, represents the most basic application

of inferential statistics and often underlies more advanced analyses. Hypothesis testing assumes that the underlying data follow an established distribution, such as the normal 
(Gaussian) distribution and that the test statistic also follows an established distribution under the null hypothesis. For example, the t-test assumes the data follow a normal distribution and the t-statistic follows a t-distribution. Using these assumption, it is possible to compare values to the mean of a population or sample and determine if they are sufficiently different such that any deviation from the mean could not be reasonably attributed to random fluctuations in the data.

To help ensure that bias does not lead to false results, it is necessary to define two hypotheses. The first is the null hypothesis, $H_{0}$, that there is no effect or that there is no difference between two measures. The other is the alternative hypothesis, $H_{1}$, which says that there is a real effect or difference which cannot be attributed to chance. In hypothesis testing, you do not seek to prove the alternative hypothesis, instead you seek to find sufficient evidence to reject the null hypothesis. Only when there is sufficient evidence to reject the null hypothesis can you say that there is support for the alternative hypothesis.

One of the most basic version of hypothesis testing is known as the $z$-test which assumes a normal underlying distribution to the data. Using a one-sample $z$-test, it is possible to test if a value is sufficiently different from the mean by calculating a $z$-score, Eqn 2.6, which measures the number of standard deviations the measured value is from the mean.

$$
z=\frac{\bar{X}-\mu}{S E_{\bar{X}}}
$$

where $S E_{\bar{X}}$ is the standard error of $X, \bar{X}$ is the mean value of $X$ and $\mu$ is the population mean. Given the $z$-score it is possible to calculate the probability that the value is different from the mean. For example, if 100 samples were drawn, the probability represents the 
expected proportion of the sample means which would be further from the population mean than the value selected. This probability is known as the $p$-value and as a general "ruleof-thumb" a $p$-value of $p<0.05$ is considered the threshold for significance [41]. Though a measure of significance, the $p$-value was never intended as a final statistic indicating a meaningful result, but instead was developed to serve as a guide to researchers that a result is potentially meaningful. Unfortunately, much research relies on finding significant $p$-values as the end result and the practice of $p$-hacking, wherein researchers resample or otherwise manipulate their results to achieve a $p<0.05$ has become more prevalent [42]. It is therefore necessary to take additional steps when a significant $p$-value is found such as determining the effect size of the result.

\subsubsection{Effect Size}

The effect size provides a measure of the magnitude of an effect. Often with large data sets, effects will be detected as statistically significant even when the size of the effect is small. This work uses Cohen's $d$, given by Eqn 2.7. Cohen's $d$ measures the mean difference in terms of the standard deviation. Cohen suggests a value of $d>0.2$ as a small effect, a value of $d>0.5$ a medium effect and any value $d>0.8$ a large effect. When the variables

are standardized, Cohen's $d$ is related to the z-score, representing the number of standard deviations from the mean of the measure [43].

$$
d=\frac{\mu_{1}-\mu_{2}}{\sigma}
$$


Here $\mu_{1}$ and $\mu_{2}$ represent the means of the two groups which are being compared. There are many other effect sizes which will not be summarized here.

\subsubsection{Error}

In statistics, errors are classified into two categories: Type I and Type II. A Type I error is made when a true null hypothesis is incorrectly rejected, leading to a false positive result. The acceptable rate of Type I errors is given by the criterion of significance $\alpha$ which is the percentage of experiments in which a Type I error would occur. This is directly related the $p$-values discussed earlier and the $\alpha$ value represents the $p$-value significance cutoff. For example, an " $\alpha$ " of 0.05 indicates that a $5 \%$ chance of Type I errors is acceptable and corresponds to a $p$-value $<0.05$. Type II errors are the opposite of Type I errors and are defined as failing to reject a false null hypothesis, a false negative result. The probability of a Type II error is given by $\beta$ and the statistical power is given by $1-\beta$ or the probability that the null hypothesis is rejected given a true alternate hypothesis. Balancing the probability of encountering a Type I or Type II error is a vital part of any experimental design [41].

\subsubsection{Bootstrapping}

Bootstrapping overcomes a limitation inherent to hypothesis testing in that the distribution of the population no longer needs to be assumed. Instead bootstrapping will calculate the statistic of interest many times using sub-samples (with replacement) of the sample data set. The central limit theorem ensures the results of thousands of tests on the bootstrapped sub-samples will approximate a normal distribution allowing the estimation of the statistic of interest as well as the confidence intervals [44]. 


\section{Part I}

\section{Conceptual Survey Evaluation}


The two following chapters evaluate the complex nature of student understanding of physics. Understanding of the results of conceptual inventories requires a theoretical basis for the structure of student knowledge. The work which follows pioneered a new method to model the structure of physics knowledge and the computational tools to characterize and optimize these models.

Chapter 3 focuses on the knowledge structure of the Force Concept Inventory (FCI) and is a mostly unaltered transcript of a published work [45]. Some sections related to the FCI which can be found in Chapter 1 were removed and some of the wording was changed to improve the flow of this thesis. Chapter 4 presents a follow-up study of the Conceptual Survey of Electricity and Magnetism (CSEM) which has been accepted for publication. The overlapping methods and theoretical structure associated with the study of the FCI were removed for brevity and the reader is directed to Chapter 3 for a review of these topics. This work was conducted and submitted for publication in collaboration with Dr. John Stewart, Dr. Seth Devore, and Dr. Gay Stewart. 


\title{
Chapter 3
}

\author{
Evaluation of the Knowledge Structure Measured by \\ the Force Concept Inventory
}


This chapter explores the knowledge structure of the FCI using Multidimensional Item Response Theory (MIRT). A substantial number of studies have attempted to understand the overall structure of the FCI. These have included purely exploratory or descriptive methods such as factor analysis [46-48], module analysis [49], cluster analysis [50, 51], item response theory $[19,52-54]$ and item response curves $[55,56]$ The structure of student reasoning on the FCI has also been investigated by methods such as model analysis that require the input of a partial model of the concepts measured by the FCI [57]. Model analysis was later shown to be exact only in certain limiting cases [51].

The reliability and validity of the FCI have also been tested. The internal consistency of the FCI measured by Cronbach's alpha is quite strong $(>0.85)[18,19]$. The instrument has also demonstrated good test-retest reliability, producing the same responses when students are given the test again [58]. While some validity issues have been identified [19], these are minor compared to those reported for some other instruments [59].

\subsection{Factor Analysis and the FCI}

The authors of the FCI provided a detailed description of the physical concepts each item in the original instrument was designed to measure [13]. Soon after its publication, attempts to extract the suggested structure with Exploratory Factor Analysis (EFA) were unsuccessful leading to debate about what the instrument actually measured [46, 60, 61]. Huffman and Heller reported that, for a sample of 145 high school students, principle component analysis identified two factors: Newton's 3rd law and Kinds of Forces. For 750 university students, only one factor was identified: Kinds of Forces. This study selected 
the number of factors by requiring that each new factor explain at least $5-10 \%$ additional variance. The difference in the number of factors identified between the Huffman and Heller study and other studies of the FCI may have resulted from the use of different criteria to identify the optimal number of factors. Methods to identify the optimal number of factors are discussed in Sec. 3.6.5.

Scott, Schumayer, and Gray applied EFA to the FCI post-test scores of a sample of 2,150 students in a college algebra-based physics course [47]. The FCI was delivered electronically and students were given no special incentive for completion. They found a single factor explained a substantial portion of the variance, but concluded a five-factor model was optimal. Parallel analysis was used to select the optimal number of factors. The "knee" of their Scree plot (a plot of the number of eigenvalues vs the total variance explained) suggested that two or three factors could also be considered optimal. In examining the loadings on the single factor, they discuss the possibility of very difficult items not being strongly correlated with the single-factor solution. The variance explained by the addition of each new factor is not reported, and therefore, the number of factors selected cannot be compared with Huffman and Heller's solution [46].

Semak et al. explored the evolution of the structure of student thinking on the FCI using factor analysis [48] for 427 algebra- and calculus-based introductory physics students. They found the optimal solution had 5 factors on the pretest and 6 factors on the post-test. Parallel analysis was used to select the optimal number of factors; however, examination of the Scree plots from their study suggests one could have also identified one or two factors as optimal for both the pretest and post-test. This would have provided support for Huffman and Heller's model. We provide a comparison of the four reported factor structures in Sec. 
3.9.

Factor analysis has also been used to investigate other sets of physics problems. Ramlo [62] calculated the factor structure of the FMCE [15], finding 3 factors for the pretest; however, these factors contained a mixture of concepts and Ramlo concluded the pretest factor structure was undefined. Three factors were also found for the post-test with items covering similar conceptual topics largely loading onto the same factor. Ramlo used a Scree plot with an eigenvalue cutoff of 2.5 to determine the optimal number of factors.

\subsection{Item Response Theory and the FCI}

Item Response Theory (IRT) contains a broad set of statistical models which calculate the probability of a student with some overall proficiency or ability to answer individual items on a test correctly. Many different IRT models have been used to investigate the FCI including the Rasch model, the 2-parameter logistic (2PL) model, the 3PL model, and MIRT. These models are reviewed in Sec. 3.6.

Many studies have investigated the FCI with IRT using a single ability parameter (unidimensional IRT). Wang and Bao employed the 3PL IRT model to investigate the FCI pretest for 2,802 college students taking calculus-based physics [52]. They reported excellent model fit with all items showing reasonable difficulty parameters and no items with negative discrimination parameters. The $3 \mathrm{PL}$ model adds a parameter to the $2 \mathrm{PL}$ model to account for random guessing. The majority of the guessing parameters were less than the $20 \%$ random guessing would produce. The use of the 3PL model for distractor-driven instruments, like the FCI which has many alternative answers based on common misconceptions, has been 
questioned [56].

Planinic, Ivanjek, and Susac performed a Rasch analysis of 1,676 Croatian high school students who had completed an algebra-based physics class [53]. The Rasch model difficulty parameters were largely in agreement with the overall item average. This study is difficult to generalize because the overall score on the instrument $(27.7 \%)$ was so low and the measurement was performed two and one-half years after instruction.

Osborn Popp, Meltzer, and Megowan-Romanowicz also used Rasch Model IRT for a sample of 4,775 high school students to investigate item fairness; all students had been taught using Modeling Instruction [54]. IRT using the Rasch model was used to determine if items within the FCI were of equal difficulty for men and women. They found that 2 of items were significantly easier for male students and 1 was significantly easier for female students.

Traxler et al. [19] also investigated item fairness in the FCI with IRT using the 2PL model. They found that eight items were substantially biased toward men and and two toward women; they proposed a reduced 19-item instrument to eliminate all biased and poorly functioning items.

Han et al. used the 3PL IRT model as part of the process of evaluating the equivalence of two shorter versions of the FCI [63]. Traxler et al. [19] cautions that the gender unfair items were not evenly distributed between the shortened tests, and therefore, the two shorter tests might have different performance results for men and women.

Scott and Schumayer [64] attempted to replicate the work of Scott, Schumayer, and Gray [47] on a related dataset using MIRT. They confirmed the 5-factor solution. Comparing the factor models of the two studies showed very good, but not perfect, agreement suggesting MIRT and EFA are complementary techniques. To select the optimal number of factors, the 
Akaike Information Criterion (AIC) and Bayesian Information Criterion (BIC) (described in Sec. 3.6.2) were minimized.

IRT has also been used to explore other sets of physics problems. Lee et al. [65] used 2PL IRT to examine how the skill of physics students using an online homework system changed between their first and second attempts at a problem. Whether feedback was given on the first attempt and the type of feedback strongly influenced the change in student skill (IRT ability) between the first and second attempt.

Morris et al. [55] introduced an alternative to IRT (bearing a very similar name), Item Response Curves (IRC), which was used to analyze the FCI. IRC analysis simplifies IRT analysis by using the overall test score as a surrogate for student ability, greatly reducing computational demands and allowing intuitive exploration of the effect of distractors. Using a sample of over 4,500 students drawn from multiple institutions, a later study by Morris et al. [56] compared IRC analysis to the IRT analysis of Wang and Bao [52] and found excellent correlation between the difficulty parameters of the models.

\subsection{The Structure of Knowledge}

Most explorations of the structure of the FCI have focused on determining a general structure which represents the entire instrument in terms a small number of factors/clusters. This reductionism is at odds with a large body of research suggesting students' knowledge of physics is complex and that students (novices) do not possess the strongly integrated view of physics of expert practitioners. Experts and novices categorize problems differently; novices by surface features and experts by deeper conceptual divisions [66, 67]. One commonly 
accepted difference in the knowledge structure of experts is the hierarchical nature of the structure, with the most fundamental principles at the top and less fundamental concepts branching out from there [68-70]. This more deliberate structuring of knowledge allows experts to engage more efficiently in chunking of knowledge [71-73] for more expedient application of the correct physics principles when engaging in problem solving.

Conversely, novices lack this deliberate knowledge structure leading to less deliberate methods of problem solving. This review will follow the categorization of expert/novice research presented in Docktor and Mestre's extensive synthesis of PER [3]. One view regarding some of the novel ways that novices approach problems differently from experts is the "misconceptions" view. This view argues that students, through their life experiences, have developed theories regarding how the world works and that using these often incorrect theories leads to some of the common difficulties in physics problem solving [10, 11, 74]. Research into these misconceptions has shown that they are very difficult to overcome due in part to the time students have spent believing them to be true $[75,76]$. Another method of explaining the differences is the "ontological categories" view, which posits that students miscategorize their knowledge, storing it in incorrect broad categories (i.e. thinking of force as a thing that can be used up) [77-79]. Another popular theoretical framework is the "knowledge in pieces" view [80-82] wherein student understanding consists of a number of granular facts that are activated, either individually or in small groups, to synthesize a solution. Regardless of the theoretical framework used to describe it, novice knowledge and the associated problem solving techniques have been shown to be highly sensitive to the context of the problem and how it relates to problems they have seen in the past [83-85]. As such, the knowledge state of students may be better described by models of a granular 
knowledge structure instead of the integrated models implied by factor analysis or cluster analysis.

The current work will produce a fine-grained model of the information needed to solve FCI problems. This model is very similar to models produced by a paradigm of cognitive research into complex problem solving pioneered by Simon and Newell [86]. This paradigm and its history, which dominated research into problem solving for over 30 years, were summarized by Ohlsson [87]. The paradigm constructed computational models that replicated the problem solving sequence of human solvers; the sequence of the human solver was identified by coding extensive think-aloud transcripts. This method was applied to examine expert/novice differences in problem solving in kinematics and dynamics, as well as other fields $[88,89]$. Reif and Heller offered a related detailed model of problem solving in mechanics [71]; this model did not meet the test of being computationally functional, but was meant to be a complete model that could serve as a prescription of expert behavior. The model we will propose for the FCI shares many features with the computational models of Larkin et al. [89] and the model of Reif and Heller [71]. The work on complex problem

solving focussed primarily on quantitative solutions; however, the framework presented by Reif and Heller acknowledged the role of qualitative decisions in the solution process and suggested extensions to model qualitative reasoning.

\subsection{Research Questions}

This chapter seeks to answer the following research questions.

RQ1: What factor structure is extracted for the FCI by MIRT? Is this structure consistent 
with the results of other factor analysis?

RQ2: Can parts of this factor structure be explained by factors other than the structure of student knowledge of Newtonian mechanics?

RQ3: If blocked items and repeated reasoning groups are removed, is the resulting factor structure consistent with Newtonian mechanics?

RQ4: Can theoretically constrained MIRT produce a model of the physical constructs measured by the FCI? If so, what is the optimal model of the FCI for this student population?

RQ5: Does the structure proposed by the FCI's authors provide a superior description of the instrument to the optimal model identified by MIRT?

This work leaves two important areas of analysis for future research: the role of misconceptions and bias. The FCI was constructed so that the distractors represented common misconceptions. In the analysis in this paper, only the correctness of the responses was analyzed. MIRT could be extended to include factors representing common misconceptions to determine how the models presented in this work would be modified.

There is a substantial body of research indicating that some problems within the FCI are unfair to women with a few unfair to men. These problems have often factored together in previous analysis $[47,48]$ leading to the possibility that some factors are identified because of biases in the problems. While this study will not focus on gender fairness, the reduced fair 19-item FCI proposed by Traxler et al. [19] was examined using the optimal theoretical FCI model identified by MIRT. For a review of research into FCI item bias see Traxler et al. [19]. For a review of the issue of gender disparities in conceptual inventories see Madsen, 
McKagan, and Sayre [90] or Henderson et al. [91]. For a general review of gender in physics see Traxler et al. [92].

\subsection{Sample}

The sample of FCI post-test results was collected from a large, southern, land-grant university with an enrollment of approximately 25,000 students. This university held a Carnegie Classification of "Highest Research Activity" for the period studied. The sample was comprised of 4,716 complete post-test responses collected from the spring 2002 semester to fall 2012 semester (23.1\% women). The demographics of the university in 2012 were $79 \%$ White, $5 \%$ African American, $6 \%$ Hispanic, and 3\% or less of other groups. The 25th to 75th percentile range of the general student population's ACT scores was 23-29 [93]. This sample was also used in the analysis of Traxler et al. [19].

The sample was collected in the introductory calculus-based mechanics course serving future physical scientists and engineers. Students in the course were required to attend two 50-minute lectures and two 2-hour laboratories each week. The lectures were largely traditional with attendance monitored by a quiz given at the beginning and end of each session. The lab sessions featured a mixture of activities including teaching assistant (TA) led interactive demonstrations, small group problem solving, inquiry-based hands-on activities, and traditional experiments. The class had been revised previous to the beginning of data collection and was presented with few changes over the period studied. The class was managed by the same lead instructor for the period studied; this instructor taught $75 \%$ of the lecture sections and oversaw the instruction of the remaining sections. 


\subsection{Factor Analysis Using Item Response Theory}

\subsubsection{Unidimensional Item Response Theory}

Unidimensional IRT uses a logistic function to model the effect of a single latent trait called "ability" on the probability of a student successfully answering an item [94]. The simplest form of IRT is called the Rasch model where the probability, $\pi_{i j}$, of a student $i$ correctly answering an item $j$ is given as a function of the latent trait $\theta_{i}$ and the item difficulty, $b_{j}$. The Rasch model is often extended by the addition of a discrimination, $a_{j}$, for each item to form the 2 Parameter Logistic (2PL) model in Eqn. 3.1:

$$
\pi_{i j}=\frac{\exp \left[a_{j}\left(\theta_{i}-b_{j}\right)\right]}{1+\exp \left[a_{j}\left(\theta_{i}-b_{j}\right)\right]} .
$$

The Rasch model is the $2 \mathrm{PL}$ model with the discrimination constrained to one, $a_{j}=1$. This model can be further extended to the 3PL model which includes a parameter for random guessing. The 3PL model has also been used to understand the properties of the FCI [52].

\subsubsection{Model Fit Statistics}

IRT uses maximum likelihood (ML) estimation techniques to determine model parameters. The model is used to calculate the likelihood function $L$ representing the probability that a specific observation occurred given the model. ML techniques iteratively search the parameter space for the values of the parameters which maximize $L$. To determine if maximum likelihood models fit the data well, several statistics have been developed and should be used in conjunction to evaluate models [95] such as the AIC, BIC, the Root Mean Square Error of Approximation (RMSEA), the Comparative Fit Index (CFI), and Tucker- 
Lewis Index (TLI).

Both AIC and BIC measure the relative information lost when using the model in comparison to the "true" model and correct for overfitting as additional parameters are added to a model $[96,97]$. Smaller AIC or BIC represent better fitting models. The definitions of AIC (Eqn. 3.2) and BIC (Eqn. 3.3) follow

$$
\begin{gathered}
A I C=2 k-2 \ln (L), \\
B I C=k \ln (n)-2 \ln (L),
\end{gathered}
$$

where $n$ is the sample size and $k$ is the number of parameters estimated. When interpreting AIC differences, Burnham and Anderson [96] recommend a difference of greater than 2 as significant and the model with significantly lower AIC should be selected. BIC follows a similar rule with Raftery defining differences of $\triangle B I C \leq 2$ as "weak," $2<\Delta B I C \leq$ 6 as "positive," $6<\Delta B I C \leq 10$ as "strong," and $\Delta B I C>10$ as "very strong" [98]. Both methods penalize the additions of parameters with BIC doing so more strongly and representing a more conservative estimate. Because of the similarity of the two measures, we will adopt Raftery's convention for both AIC and BIC. The likelihood, $L$, is the probability that the measured data would be observed given some model, in this case MIRT. For most multiple parameter models with a large sample, this probability is very small. AIC and BIC primarily depend on $-\ln (L)$ which tends to be large because $L$ is very small. As such, changes in AIC and BIC represent exponential changes in the probability of the observed data being represented by the model. If the sample size and number of parameters is constant, 
then a reduction of $\mathrm{AIC}$ or $\mathrm{BIC}$ by $10, \triangle A I C=-10$ or $\triangle B I C=-10$, means that the lower AIC or BIC model is $e^{5}=148$ times more likely.

The RMSEA is a badness-of-fit statistic with values ranging from 0 to 1 . Interpretation of the RMSEA relies on an analysis of the $90 \%$ confidence intervals (CI) of the statistic. When using the RMSEA, three hypotheses are tested. The first is the exact fit hypothesis $\mathrm{H}_{0}: \mathrm{RMSEA}=0$, which is rejected if the lower bound of the CI excludes zero. The second is the "not-close-fit" hypothesis $\mathrm{H}_{0}$ : RMSEA $\geq 0.05$, which is rejected if the upper bound of the CI is $\leq 0.05$ thus indicating a close fitting model. Finally the "poor-fit" hypothesis $\mathrm{H}_{0}: \mathrm{RMSEA} \geq 0.10$ is rejected if the upper bound on the CI is less than 0.10 [99]. The statistical software used in this analysis reports the more common $95 \%$ confidence interval; we will use this more conservative test in our analysis.

The final two fit statistics reported are closely related: the CFI and TLI. .These quantities are incremental-fit-index goodness-of-fit statistics which measure the departure of the tested model from the null model assuming independence, that all parameters are uncorrelated. The equations for CFI and TLI are given in Eqn 3.4 and Eqn. 3.5 respectively.

$$
\begin{gathered}
C F I=1-\frac{\max \left[\chi_{t}^{2}-d f_{t}, 0\right]}{\max \left[\left(\chi_{t}^{2}-d f_{t}\right),\left(\chi_{i}^{2}-d f_{i}\right), 0\right]}, \\
T L I=\frac{\left(\chi_{i}^{2} / d f_{i}\right)-\left(\chi_{t}^{2} / d f_{t}\right)}{\left(\chi_{i}^{2} / d f_{i}\right)-1}
\end{gathered}
$$

Where $\chi_{i}^{2}$ is the chi-square model of independence for the baseline null model, $\chi_{t}^{2}$ is the same but for the researcher designed model, $d f$ is the degrees of freedom for each model. There exists some debate as to the appropriate cutoff values for good fit using the CFI and TLI with 
Kline [99] recommending 0.90 as the minimum for acceptable model fit while other authors $[95,100]$ recommend that a CFI or TLI of 0.97 or greater represents a good fit relative to the independence model and 0.95 or greater represents an acceptable fit. We will use the more conservative and more common 0.95 cutoff for acceptable model fit.

\subsubsection{Multidimensional Item Response Theory}

Unidimensional IRT uses a single ability trait; however, conceptual inventories like the FCI are designed to probe multiple topics such as kinematics, forces, and momentum. MIRT extends the IRT model to include multiple latent ability traits. If $k$ latent traits are to be modelled, then student $i$ 's ability becomes the $k$ component vector $\boldsymbol{\theta}_{i}$. Each item has $k$ discrimination parameters given by the vector $\boldsymbol{a}_{j}$. MIRT models can be constructed in two forms: compensatory and non-compensatory. The compensatory form of MIRT assumes that the solution does not depend on the latent traits independently and that a deficiency in one trait can be compensated for by a strength in one of the other traits. The compensatory MIRT model is shown in Eqn. 3.6:

$$
\pi_{i j}=\frac{\exp \left[\boldsymbol{a}_{j} \cdot \boldsymbol{\theta}_{i}+d_{j}\right]}{1+\exp \left[\boldsymbol{a}_{j} \cdot \boldsymbol{\theta}_{i}+d_{j}\right]},
$$

where $d_{j}$ is related to the difficulty of the item and is assumed to be the same for each of the latent traits. In the $2 \mathrm{PL}$ model, $d_{j}=-a_{j} b_{j}$. Conversely, the non-compensatory model limits the degree to which one latent ability can compensate for the lack of another. This model does not assume the same difficulty for each item and provides an independent difficulty for each latent trait. Non-compensatory models require a doubling of the parameters estimated 
and, in our analysis, these models failed to converge.

\subsubsection{Exploratory and Confirmatory MIRT}

MIRT can be used as both an exploratory and confirmatory method. For exploratory analysis, the discrimination matrix $\boldsymbol{a}$ is not constrained and each element may take on any value. For each item $j, \boldsymbol{a}_{j}$ is a vector of length $k$. These vectors over all items form a matrix with elements $a_{j k}$. Each column in this matrix, $\boldsymbol{a}_{k}$, represents a "factor." The number of factors in the model is incrementally increased. Successive models are compared using fit statistics to identify the optimal number of factors. A confirmatory analysis begins with the selection of a theoretical model. The model identifies a small number of concepts covered by each item. Each concept is associated with a column $k$ in $\boldsymbol{a}$. If item $j$ is not associated with concept $k$ in the theoretical model, the discrimination is constrained to be zero, $a_{j k}=0$, for the item. The constrained model is then fit to the data and model fit statistics are examined. If fit is acceptable, a small number of related models are then explored to determine the optimal model. When conducting a confirmatory MIRT analysis with the intent of exploring the potential factor space, it is important to define a set of models to be investigated before fitting the initial model.

\subsubsection{Additional Analyses}

While MIRT allows statistical selection of the optimal number of factors, traditional EFA uses a number of non-statistical criteria. Factor selection often begins by an examination of the "Scree" plot which plots the eigenvalue of the correlation/covariance matrix corresponding to the factor against the factor number; the eigenvalue is related to the variance explained by 
the factor. One, then, identifies the "knee" of the Scree plot, the point of greatest curvature. The number of factors corresponding to the knee is the optimal number of factors. For factor numbers greater than the location of the knee, each additional factor explains substantially less variance than the factors already extracted.

Many additional methods have been developed and often yield contradictory results. The sum of the eigenvalues of the correlation/covariance matrix is equal to the trace of the matrix; therefore, an eigenvalue that is less than the mean correlation/covariance represents a factor that explains less variance than an individual item. The optimal number of factors can then be extracted as the last factor with eigenvalue above the mean. Parallel analysis computes the eigenvalues of a random correlation matrix; the optimal factor number is the last factor with eigenvalue greater than the parallel analysis eigenvalue.

Partial correlation matrices will be reported. The partial correlation matrix for the dichotomous scores on individual FCI items was calculated by regressing the total FCI score on the individual item score using a general linear model. The correlations of the residuals of these regressions form the partial correlation matrix.

The mean and standard deviation of MIRT parameters, $\boldsymbol{a}_{\boldsymbol{j}}$ and $d_{j}$, were calculated by bootstrapping. Bootstrapping is a statistical technique that allows the calculation of the average, standard deviation, and confidence interval for a dataset without assuming a statistical model. This is done by forming sub-samples of the data with replacement and recalculating the desired parameter for each sub-sample. For this work, 200 sub-samples were used; this calculation required one week of computational time on a modern personal computer.

All statistical analyses were carried out in the "R" software package [101]. MIRT was 
performed with the "mirt" package [102]. Nested MIRT models were compared using the anova function which performs a likelihood ratio test. This work used correlation analysis to investigate the origin of the factor structures extracted. The correlation matrix was presented in a visualization rendered by the "qgraph" package [103]. Partial correlation matrices were constructed by using the "glm" function to regress the total FCI score on the dichotomous scores of the individual items. Factor analysis was carried out with the "factanal" function in the "stats" package. The "nFactors" package was used to generate the Scree plot and to perform parallel analysis. Bootstrapping was performed with the "boot" package [104, 105].

\subsection{Results - Exploratory Analyses}

The FCI was first examined with MIRT without employing a theoretical model, thus performing an exploratory factor analysis (EFA). Exploratory methods extract models from data without the constraints of an imposed model. Correlation analysis was then used to understand the resulting factor structure. Expert solutions of the FCI were then used to construct a theoretical model of mechanics which allowed further exploration of the correlation structure. In Sec.3.8, the work shifts to a confirmatory analysis using MIRT to explore how the theoretical model mapped onto student responses to the FCI. Finally, the model proposed by the FCI authors was fit and compared to the optimal model identified in this work.

\subsubsection{Exploratory Factor Analysis}

MIRT was used to perform EFA on the FCI. Models with progressively more factors were fit and compared using a likelihood ratio test which computes a chi-squared statistic. 
A 9-factor model improved model fit over an 8-factor model $\left[\chi^{2}(22)=53.44, p<0.001\right]$ and explained $56 \%$ of the variance in the item scores. The last factor added explained $3.6 \%$ additional variance. The 10-factor model did not significantly improve model fit. The 9factor model showed a very strong improvement in AIC and BIC on both the 8-factor and 10-factor model using Raftery's classification [98]. The 9-factor model (varimax rotation) is shown in Table 3.1. Varimax rotation adjusts covariance matrix such that all of the factors are orthogonal to each other. Varimax is a commonly applied rotation, however other rotations that do not require complete orthogonality do exist. Previous studies investigating the structure of the FCI began their analyses with varimax rotations, [47, 48]. Factors are reported as columns and labelled "FC." The table also identifies the FCI problem blocks. The table reports, $d ; d$ is related to the overall difficulty of the item (Eqn. 3.6). Easier items have larger $d$. The value for $d$ represents the factor loading, a measure of the degree to which an factor is measured by that item. Loadings greater the 0.7 are bolded. Loadings between 0.5 and 0.7 are italicized.

While the 9-factor model was statistically superior, the model fit statistics shown in Table 3.2 did not provide a clear identification of the number of factors. While the 9-factor model is statistically significantly better than all other models, there was not a significant improvement from the 6-factor model to the 7-factor model $\left[\chi^{2}(24)=32.79, p=0.109\right]$. The 9-factor model was a significant improvement over the 6 -factor model $\left[\chi^{2}(69)=196\right.$, $p<0.001]$. The 5 -factor model had superior RMSEA, CFI, and TLI statistics. While the 9-factor model minimized AIC, the 6-factor model minimized BIC. The "knee" in the Scree plot calculated using traditional EFA, shown in Fig 3.1, suggests 3 to 4 factors. As such, after 3 factors are extracted, it is difficult to make a definitive case for the number of factors. 
Table 3.1: Factor Loadings for Exploratory Factor Analysis with Multi-dimensional IRT (varimax rotation). Loadings greater the 0.7 are bolded. Loadings between 0.5 and 0.7 are italicized.

\begin{tabular}{|c|c|c|c|c|c|c|c|c|c|c|}
\hline \# & FC1 & FC2 & FC3 & FC4 & FC5 & FC6 & FC7 & FC8 & FC9 & $\mathrm{d}$ \\
\hline 1 & & & & & & & & & 0.78 & 8.08 \\
\hline 2 & & & & & & & & & 0.42 & 0.90 \\
\hline 3 & & -0.54 & & & & & & & & 3.36 \\
\hline 4 & & & 0.88 & & & & & & & 1.38 \\
\hline \multicolumn{11}{|c|}{ Block 5-6 } \\
\hline 5 & & & & & & -0.71 & & & & 0.63 \\
\hline 6 & & & & & 0.78 & & & & & 4.81 \\
\hline 7 & & & & & 0.64 & & & & & 2.81 \\
\hline \multicolumn{11}{|c|}{ Block 8-11 } \\
\hline 8 & & -0.56 & & & 0.35 & & & & & 3.38 \\
\hline 9 & & -0.63 & & & & & & & & 2.18 \\
\hline 10 & & -0.53 & & -0.32 & & & & & & 4.14 \\
\hline 11 & & -0.58 & & & & & & & & 1.81 \\
\hline 12 & & & & & 0.33 & & & -0.44 & & 3.40 \\
\hline 13 & & -0.63 & & & & -0.41 & & & & 3.40 \\
\hline 14 & -0.35 & & & & & & & -0.47 & & 1.01 \\
\hline \multicolumn{11}{|c|}{ Block 15-16 } \\
\hline 15 & & -0.52 & 0.64 & & & & & & & 0.91 \\
\hline 16 & & -0.39 & 0.35 & -0.43 & & & & & & 3.89 \\
\hline 17 & & & & -0.74 & & & & & & 0.37 \\
\hline 18 & & & & & & -0.81 & & & & 0.70 \\
\hline 19 & & -0.58 & & & & & & & & 2.73 \\
\hline 20 & & & & & & & & & & 0.79 \\
\hline \multicolumn{11}{|c|}{ Block 21-24 } \\
\hline 21 & -0.74 & & & & & & & & & -0.18 \\
\hline 22 & -0.84 & & & & & & & & & 0.83 \\
\hline 23 & -0.48 & & & & 0.37 & & -0.40 & & & 2.10 \\
\hline 24 & -0.39 & & & & & & -0.50 & & & 3.96 \\
\hline \multicolumn{11}{|c|}{ Block 25-27 } \\
\hline 25 & & & & -0.86 & & & & & & 0.57 \\
\hline 26 & -0.39 & & & -0.61 & & & & & & -1.34 \\
\hline 27 & -0.36 & & & & & & & & & 1.52 \\
\hline 28 & & & 0.74 & & & & & & & 1.91 \\
\hline 29 & & & & & & & & & & 1.63 \\
\hline 30 & & & & & & & & & & 0.67 \\
\hline
\end{tabular}




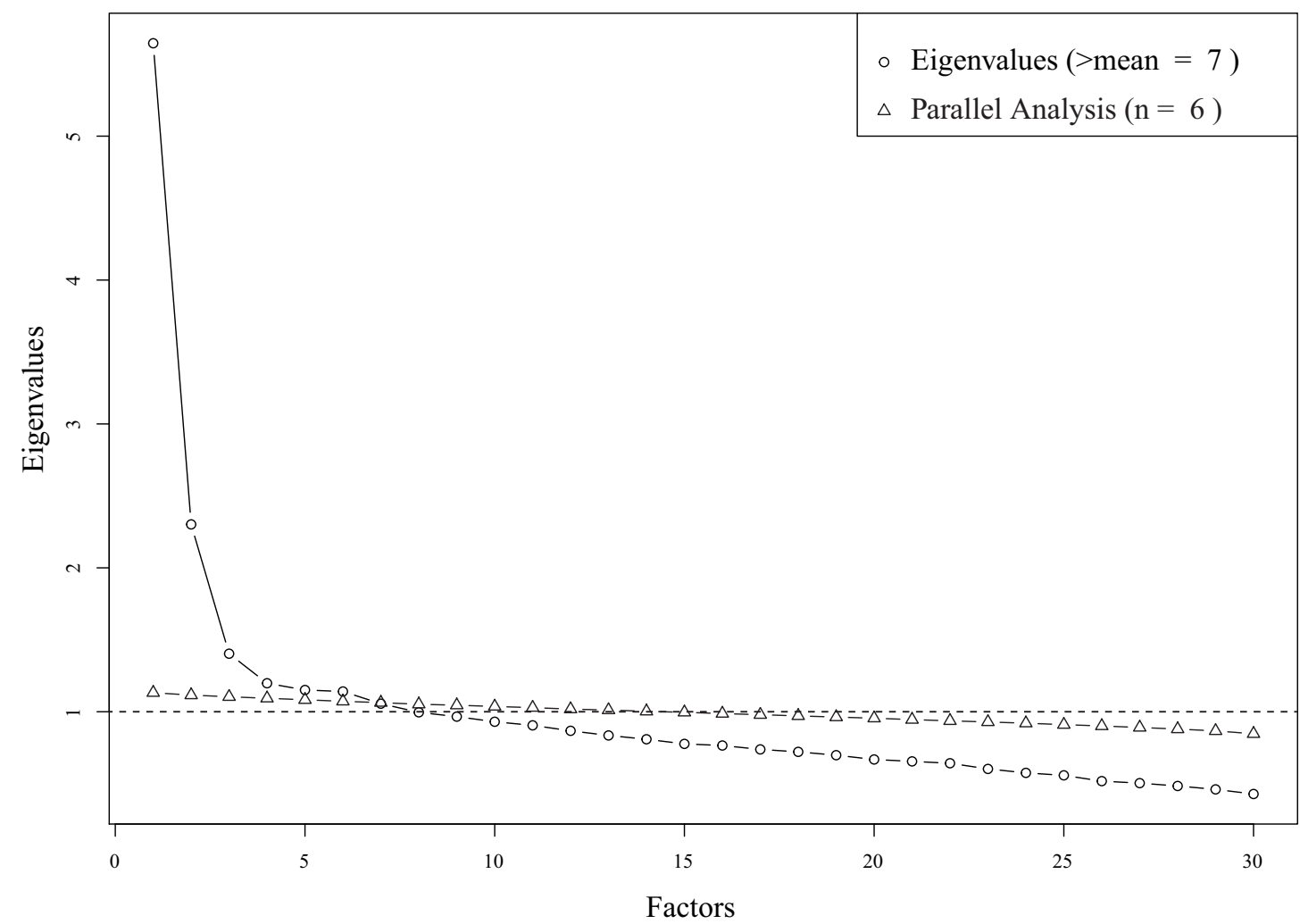

Figure 3.1: Scree Plot of the FCI. The optimal number of factors corresponds to where the plot of the number of eigenvalues against the number of factors' slope decreases significantly ("knee") and is close to an eigenvalue of 1 .

We selected the 9-factor model because it was the model identified as optimal using the likelihood ratio test, minimized AIC, and provided the greatest resolution of the structure of the instrument.

Traditional EFA was also performed. For this analysis, the criterion that the eigenvalue be greater than the mean eigenvalue suggested a 7-factor solution, parallel analysis suggested a 6-factor solution, while an examination of the "knee" in the Scree plot suggested 3-4 factors. Like other published FCI Scree plots, there was a rapid decline from 1-3 factors followed by a long tail where additional factors each explained $2-4 \%$ additional variance. If Huffman and Heller's criteria for the retained factors, which were required to explain $5-10 \%$ of the 
Table 3.2: MIRT fit statistics

\begin{tabular}{cccccc} 
Factors & AIC & BIC & RMSEA & TLI & CFI \\
\hline 1 & 132,042 & 132,430 & 0.071 & 0.83 & 0.84 \\
2 & 128,805 & 129,379 & 0.047 & 0.92 & 0.94 \\
3 & 127,863 & 128,619 & 0.042 & 0.94 & 0.95 \\
4 & 127,223 & 128,153 & 0.038 & 0.95 & 0.96 \\
5 & 126,553 & 127,651 & 0.032 & 0.97 & 0.98 \\
6 & 126,239 & 127,498 & 0.066 & 0.85 & 0.91 \\
7 & 126,254 & 127,668 & 0.071 & 0.83 & 0.91 \\
8 & 126,192 & 127,755 & 0.067 & 0.85 & 0.92 \\
9 & 126,180 & 127,885 & 0.060 & 0.88 & 0.94 \\
10 & 126,214 & 128,055 & 0.066 & 0.86 & 0.94 \\
\hline
\end{tabular}

variance, was used [46], only two factors would have been retained. The 5-factor solution was very similar to other published solutions with many items loading on the first two factors as was also observed by Scott, Schumayer, and Gray [47]. Exploratory methods, such as factor analysis or cluster analysis, can identify structures correlated by unexpected features. The items in the first two factors in either the 5-factor EFA model or in Scott, Schumayer, and Gray do not seem strongly related by the physical principles they test, which opens the possibility that some other feature is causing the correlations which cause groups of items to be identified as factors.

\subsubsection{Correlation Analysis}

Factor analysis accomplished either traditionally or through MIRT identifies combinations of items which vary together. Co-variation of individual items can also be examined through correlation analysis. The full FCI correlation matrix contains 900 entries making it difficult to interpret; however, numerous visualizations of the correlation matrix have been created. Figure 3.2 presents one such visualization of the FCI correlation matrix created with the 
"R" qgraph package. Solid lines (green) represent positive correlations and dashed lines (red) represent negative correlations. Only correlations greater than 0.3 (Cohen's criteria for medium effect size) are shown. No pair of questions was negatively correlated with $|r|>0.3$ where $r$ is the correlation coefficient and, therefore, there are no dashed lines in the figure. The placement of nodes is calculated to be visually appealing and does not convey additional information; only the connections between nodes are important.

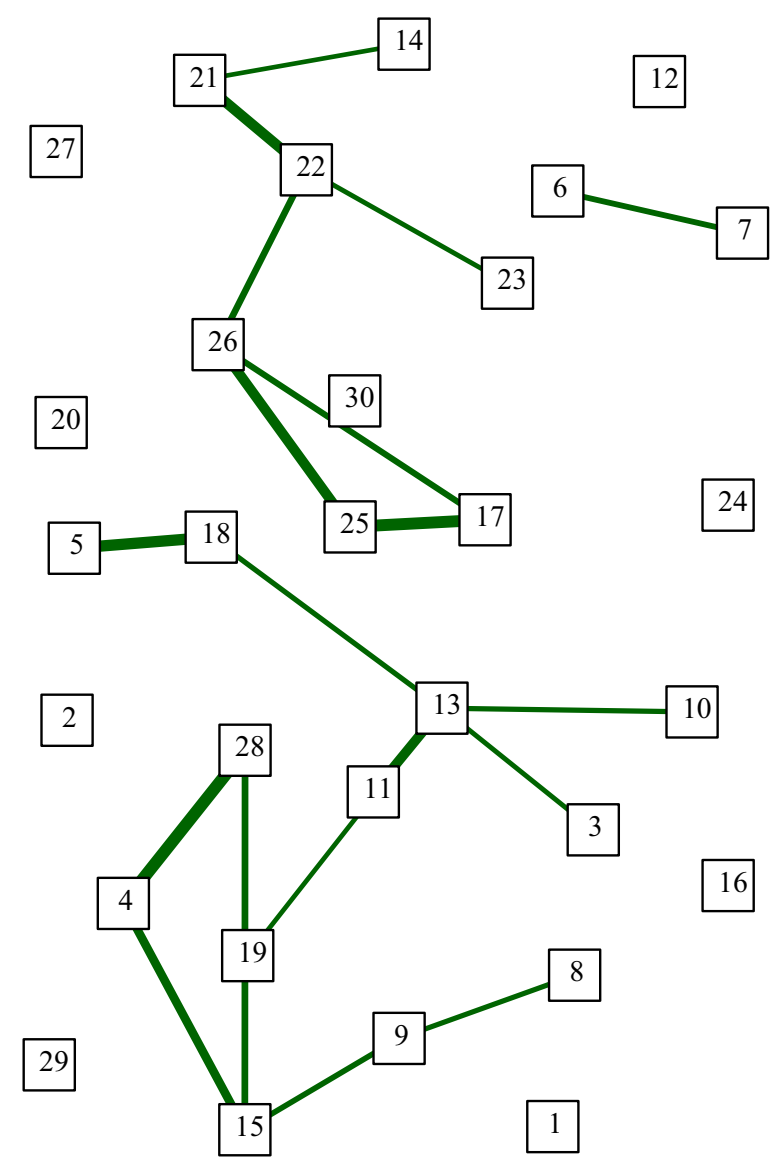

Figure 3.2: Correlation matrix for all FCI items. Lines represent correlations with $|r|>0.3$. Line thickness represents the size of the correlation. Solid (green) lines represent positive correlations; dashed (red) lines negative correlations. No negative correlations were present.

There are many potential sources of the correlations shown in Fig. 3.2. Groups of highly correlated items often form the elements of a factor with the highest loading; in some sense they "nucleate" the factor. Some correlations may arise because two items require 
similar physical principles for their solution or that they elicit the same misconception. In previous factor analysis, only these explanations of the factor structure have been considered.

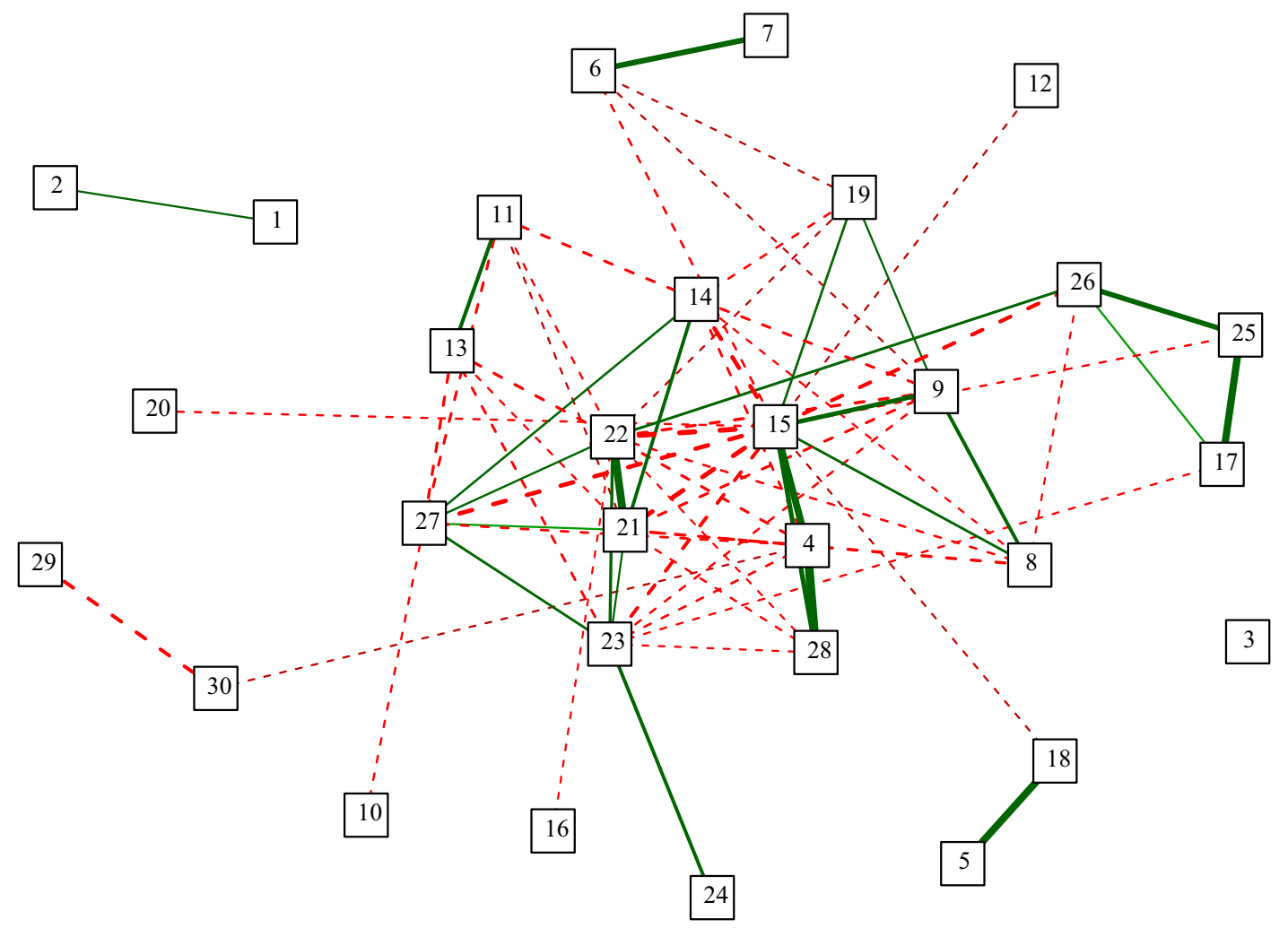

Figure 3.3: Partial correlation matrix for all FCI problems correcting for total FCI score (only $|r|>$ 0.1 shown). Line thickness represents the size of the correlation. Solid (green) lines represent positive correlations; dashed (red) lines negative correlations.

The FCI contains 4 groups of problems where each item in the group shares a common stem; we will call these groups "problem blocks." The problem blocks have been identified in Table 3.1. One additional group of items 25-27 does not share the same stem, but items 26 and 27 explicitly refer to item 25 . While blocking the problems may shorten the reading time for the student, it can also generate correlations between items that are not the result of the physical properties required for their solution. If a student misinterprets the stem, 
then this error will affect the solution of each problem in the block. An error in an earlier item in a block can cause errors in later items. Examination of Table 3.1 shows that many of the largest factor loadings in individual factors occur for problems in the same block; likewise, in Fig. 3.2 many of the most strongly correlated item pairs are part of problem blocks. An examination of the physical principles required to solve the strongly correlated blocked problems does not suggest the level of commonality demonstrated by the factor or correlation structure. As such, it seems likely that at least some of the factor and correlation structure results from the decision to use groups of problems with a common stem.

A second possible source of correlations not related to underlying physical principles is correlation through total test score. Two problems could be correlated because either only the strongest students answer them correctly or only the weakest students answer them incorrectly; they are correlated through the total test score. To remove this effect, a partial correlation matrix controlling for total test score was calculated as shown in Fig. 3.3. Examination of Fig. 3.3 shows that the problem blocks $\{8,9\},\{21,22,23,24\}$, and $\{25$, $26\}$ still stand out as highly correlated. Four other groups of questions emerge as correlated $\{5,18\},\{6,7\},\{17,25\}$, and $\{4,15,28\}$. To understand these groups, we constructed a model of the solution to the FCI, presented in the next section.

\subsubsection{A Theoretical Framework}

Hundreds of physicists have offered models of the structure of introductory mechanics either through the production of textbooks, scientific papers, or in their solution of introductory mechanics problems. We sought to produce one such model that synthesized the structure of introductory mechanics commonly presented in textbooks with the statements found in 
expert solutions of FCI problems. This resulted in a set of statements about introductory mechanics shown in Table 3.3; the statements will be called "principles" following Larkin et al. [89]. The principles were classified as Definitions (DF), Laws (L), Corollaries (C), Results (R), Facts (F), Lemmas (LM), and Reasoning (RS). Corollaries could be derived from laws, results, and definitions but required some non-trivial reasoning. A result, such as the constant acceleration kinematic equations, was derived as a special case of the laws and definitions. Knowledge of how the universe worked that did not raise to the level of a law were called facts. Expert solutions often contained specializations of the physical laws and definitions to the individual problem; these special cases were called lemmas. The FCI contains one item (item 19) which required a unique piece of reasoning (RS1) in multiple expert solutions. To solve the problem, one must argue if one quantity is constant and another begins smaller than the first quantity and ends larger than that quantity, then the quantities must be equal at some point. Many of the principles in Table 3.3 are consistent with principles used in models of physics problem solving proposed by Larkin et al. [89] and Reif and Heller [71]. The principles can be divided into two broad classes: core principles including the definitions, laws, facts, corollaries, and results and supplementary principles including the lemmas and reasoning. Without the core principles, the description of Newtonian mechanics is incomplete; supplementary principles specialize core principles to specific situations or provide specific patterns of reasoning.

To map out the subset of Newtonian mechanics tested by the FCI, a careful solution of the FCI was collected from the lead instructor who oversaw the course studied. Solutions were also collected from faculty and graduate students in the research team. These solutions were decomposed to the sentence level and each sentence classified. These statements did not 
contain many of the core principles shown in Table 3.3. Many lemmas, however, provided specializations of more general core principles not found in the expert solutions. The core principles were introduced based on the project team's understanding of Newtonian physics. For example, the expert solutions contained Lemma LM7 ("If velocity is constant, then acceleration is zero"), but did not contain the more general definition DF2 (" $\vec{a}=d \vec{v} / d t ")$ so DF2 was added to the model. A core principle was introduced for each lemma; often many lemmas were derived from a single core principle. With only a small sample, it became obvious that a complete set of supplementary principles would be very long and not particularly useful, but that the existing lemmas fit well into a well-established structure of Newtonian mechanics involving the core principles. The model of Newtonian mechanics as measured by the FCI produced by this process is shown in Table 3.3. The table also shows the core principle from which a supplementary principle can be derived and the FCI items whose solution requires the principle.

The model in Table 3.3 represents a preliminary model for understanding solutions of the FCI. It does not contain any representation of student misconceptions. The set of lemmas would almost certainly change somewhat if a different set of expert solutions were used. Some parts of the core model would be agreed upon by most experts: DF1, DF2, L2, and L3, for example. However, it is doubtful that a group of experts would agree on all elements. For example, it might be argued that Newton's 1st law is unnecessary because it can be derived from Newton's 2nd law and kinematics. Also, it might be argued that separate principles for one-dimensional kinematics $(\mathrm{C} 3$ and $\mathrm{C} 4)$ and three-dimensional kinematics (R1 and R2) are unnecessary. In Sec. 3.8, MIRT is used to explore possible changes to the model and identify the model which most strongly captures the Newtonian thinking of this student 
Table 3.3: Theoretical model of Newtonian mechanics as tested by the FCI. Principles in bold were included in the optimal model 3 fitting the reduced FCI.

\begin{tabular}{|c|c|c|c|}
\hline Label & $\begin{array}{l}\text { Derived } \\
\text { From }\end{array}$ & FCI\# & Principle \\
\hline & & & Kinematics \\
\hline DF1 & & 19,20 & Definition of velocity $(\vec{v}=d \vec{r} / d t)$ \\
\hline DF2 & & & Definition of acceleration $(\vec{a}=d \vec{v} / d t)$ \\
\hline R1 & & & Trajectory $\vec{a}=$ constant $\left(\vec{r}(t)=\vec{r}_{0}+\vec{v}_{0} t+\frac{1}{2} \vec{a} t^{2}\right)$ \\
\hline R2 & & & Velocity $\vec{a}=$ constant $\left(\vec{v}(t)=\vec{v}_{0} t+\vec{a} t\right)$ \\
\hline $\mathrm{C} 1$ & DF1 & 6,7 & Instantaneous velocity is tangent to the trajectory. \\
\hline $\mathrm{C} 2$ & DF2 & 5,18 & Objects moving in a curved trajectory will experience centripetal acceleration. \\
\hline C3 & $\mathrm{R} 1$ & & $1 \mathrm{D}$ trajectory $a=$ constant, $\left(x(t)=x_{0}+v_{0} t+\frac{1}{2} a t^{2}\right)$ \\
\hline $\mathrm{C} 4$ & $\mathrm{R} 2$ & & $1 \mathrm{D}$ velocity $a=$ constant, $\left(v(t)=v_{0}+a t\right)$ \\
\hline LM1 & DF1 & 14 & If two objects move together, they have the same initial velocity when separated. \\
\hline LM2 & $\mathrm{R} 1$ & 2 & Motion may be separated along orthogonal axes. \\
\hline LM3 & $\mathrm{C} 3$ & 2 & If motion is one-dimensional and $a=0$, then $d=v t$. \\
\hline LM4 & $\mathrm{R} 2$ & $3,22,26$ & Objects under constant acceleration with $\vec{a}$ parallel to $\vec{v}$ speed up. \\
\hline LM5 & $\mathrm{R} 2$ & 27 & Objects under constant acceleration with $\vec{a}$ opposite to $\vec{v}$ slow down. \\
\hline LM6 & $\mathrm{R} 1$ & $12,14,21$ & $\begin{array}{l}\text { Objects under constant acceleration with some initial velocity perpendicular } \\
\text { to the acceleration travel in a parabolic arc. }\end{array}$ \\
\hline LM7 & DF2 & 20 & If velocity is constant, then acceleration is zero. \\
\hline LM11 & C3 & 1,2 & $\begin{array}{l}\text { If the accelerations and initial velocities are equal, objects travel } \\
\text { the same distance in the same time. } \\
\text { Dynamics }\end{array}$ \\
\hline DF3 & & 26 & The net force is the vector sum of the forces (forces add as vectors). \\
\hline L1 & & $6,7,8,10,17,23,24,25$ & Newton's 1st law \\
\hline L2 & & $5,18,26,27$ & Newton's 2nd law \\
\hline L3 & & $4,15,16,28$ & Newton's 3rd law \\
\hline LM8 & L2 & 21 & Constant force produces constant acceleration. \\
\hline LM9 & L2 & 8,21 & If the force only has one component, an object accelerates in that direction. \\
\hline LM10 & DF3 & 17,25 & $\begin{array}{l}\text { If the net force is zero and only two forces are exerted on the object, } \\
\text { they must be equal but opposite. } \\
\text { Properties of Forces }\end{array}$ \\
\hline $\mathrm{L} 4$ & & $\begin{array}{l}1,2,3,5,11,12,13,14 \\
17,18,29,30\end{array}$ & $\begin{array}{l}\text { Objects near the earth's surface experience a constant downward } \\
\text { force/acceleration of gravity. }\end{array}$ \\
\hline F1 & & 11,29 & An object in contact with a surface experiences a normal force. \\
\hline F2 & & $11,13,18,30$ & An object does not necessarily experience a force in the direction of motion. \\
\hline F3 & & 3,29 & Air pressure does not exert a net downward force. \\
\hline F4 & & 30 & The wind can exert a force on an object. \\
\hline F5 & & 1,3 & Air resistance is negligible for a compact object moving a short distance. \\
\hline F6 & & 3 & The force of gravity is approximately constant near the earth's surface. \\
\hline F7 & & 27 & $\begin{array}{l}\text { Objects that slide across a surface experience a force of friction opposite motion. } \\
\text { Other }\end{array}$ \\
\hline DF4 & & & Magnitude of vector $\left(|\vec{A}|=\sqrt{A_{x}^{2}+A_{y}^{2}+A_{z}^{2}}\right)$ \\
\hline C5 & DF4 & 9 & Triangle inequality \\
\hline RS1 & & 19 & $\begin{array}{l}\text { If one quantity is constant and another quantity is smaller at one time and } \\
\text { larger at another time, then the two quantities must be equal at some time. }\end{array}$ \\
\hline
\end{tabular}


population.

We note that the fact F2 might be considered to specifically address the motion-impliesforce misconception. It was present in most expert solutions to eliminate specific distractors. We will find that its inclusion improves the model and future work may identify other facts that allow common misconceptions to be added to FCI models.

There were some additional minor decisions made to produce the model in Table 3.3. Item 17 has a distractor that requires the application of F3 (net downward force of the air); no expert solution included this principle and it was not included in the model of item 18 . LM4 and LM5 were written for general three-dimensional motion and are marked as derived from R2. Items 26 and 27, which use these lemmas, are one-dimensional problems. As the lemmas are folded into the principles they are derived from to produce model 3 below, the items using these lemmas will be appropriately distributed to one- or three-dimensional kinematic principles. Item 18 was coded with a centripetal acceleration implying a tension force on the boy from the rope; this item could have also been coded by introducing the tension force as an additional fact. The correlation with item 5 and the lack of any additional items using a tension force caused the selection of this coding. Law L4 and fact F6 both involve a constant force of gravity near the earth's surface. Fact F6 was introduced because FCI item 3 seems to require the student to explicitly reason that the force of gravity does not change much over the height of a single-story building.

\subsubsection{Reduced Exploratory Factor Analysis}

The theoretical model in Table 3.3 provides an explanation for some of the remaining strong correlations in Fig. 3.3 which were not explained by the block structure of the FCI. 
Items 4, 15, and 28 all require only L3 (Newton's 3rd law) for their solution. Items 17 and 25 share both L1 and LM10, items 5 and 18 share L2, L4, and C2, and items 6 and 7 share C1 and L1. Item 16 also only requires Newton's 3rd law; however, this item was not as strongly correlated with the other Newton's 3rd law items in Fig. 3.3. While Newton's 3rd law plays a central role in Newtonian mechanics and, therefore, one would expect it to be repeated multiple times in the FCI, the repetition of the other combinations of principles is difficult to support theoretically as combinations somehow central to mechanics and thus deserving special focus. The FCI authors did not discuss the choice to include the problem pairs $\{5,18\},\{6,7\}$ and $\{17,25\}$ and therefore it seems likely the inclusion of these pairs of very similar problems was accidental. The inclusion of these problems does not affect the ability of the instrument to measure an overall force concept beyond the reduction of the breadth of the instrument; however, the repetition of these problems does impact the correlation and exploratory factor structure. Figure 3.3 shows the scores on these pairs of problems are highly correlated and these pairs make up the strongest loading in factors FC4, FC5, and FC6 in Table 3.1. It seems likely that the strong correlations of the pairs was part of the reason these factors were extracted and that the factor structure could be significantly modified by removing one problem of each pair and inserting problems that repeated a different set of principles. As such, any general conclusion drawn from the existence factors FC4, FC5, or FC6 about the structure of knowledge of Newtonian mechanics is suspect.

These factors based largely on pairs of questions also serve to explain the relatively universal structure of the Scree plots reported in this and other works. The Scree plots reported all decreased strongly from 1-3 factors and then the amount of variance explained by additional factors diminishes rapidly. If a factor is mostly capturing the co-variance of 
Table 3.4: Exploratory factor analysis for the reduced FCI (varimax rotation). Only loadings greater than 0.3 are shown. Loadings greater the 0.7 are bolded. Loadings between 0.5 and 0.7 are italicized.

\begin{tabular}{lccccccc}
\hline FCI \# & FC1 & FC2 & FC3 & FC4 & FC5 & FC6 & d \\
\hline 1 & & & 0.31 & & & $\mathbf{0 . 7 5}$ & 6.25 \\
2 & & & & & & 0.65 & 0.93 \\
3 & & -0.60 & & & & & 3.35 \\
4 & & & $\mathbf{0 . 8 6}$ & & & & 1.15 \\
5 & & -0.34 & & -0.32 & & & 0.32 \\
7 & & & & -0.33 & -0.43 & & 2.28 \\
8 & & -0.48 & 0.33 & & -0.36 & & 3.06 \\
12 & & & & -0.55 & & & 2.82 \\
13 & & $\mathbf{- 0 . 7 0}$ & & & & & 3.02 \\
14 & & & & -0.67 & & & 0.65 \\
15 & & -0.47 & 0.64 & & & & 1.04 \\
17 & -0.33 & & & & & & 0.18 \\
19 & & -0.68 & & & & & 2.90 \\
20 & -0.41 & & & & & & 0.76 \\
21 & & & & -0.62 & & & -0.47 \\
28 & & & 0.81 & & & & 1.92 \\
29 & & & & & & & 1.67 \\
30 & & -0.35 & & -0.33 & & \\
\hline
\end{tabular}

two items, the amount of variance it can explain will be small.

With these observations, much of the original factor structure identified by EFA appears to be either a result of the block structure of the FCI or of repeated problems with very similar solution structure. Removing all but the first problem in each problem block and the second of the repeated problem pairs produces a reduced 18-item instrument. Because item 6 was removed due to blocking, item 7 was retained. The Newton's 3rd law items were also retained because of the centrality of this principle to Newtonian physics. The optimal MIRT model for this set of problems is shown in Table 3.4; 6 factors were optimal.

Examination of Table 3.4 shows some factors that map onto the theoretical model of mechanics. The problems have been placed in a Venn Diagram in Fig. 3.4 based on the 


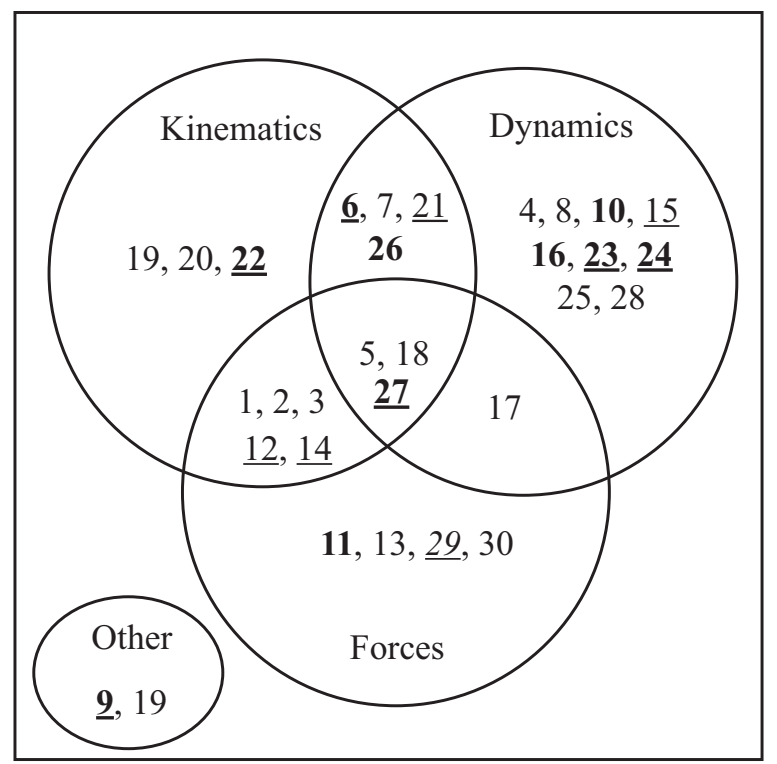

Figure 3.4: Venn Diagram of the distribution of problems in the FCI. Items in bold are the blocked items removed from the analysis. Underlined items are items identified as unfair to men or women by Traxler et al. [19] (Item 29 was identified as fair but unreliable).

general classification in Table 3.3. All FCI items have been included in the diagram. Items removed to eliminate blocking are bolded. Unfair items identified by Traxler et al. [19] are underlined; these will be discussed later. Few factors contain loadings that are localized to individual regions of the Venn Diagram. There are also loadings that cannot be supported theoretically. Factor FC3 contains the Newton's 3rd law items, but it also loads on items 1 and 8 which have nothing to do with Newton's 3rd law. Likewise, item 15, which requires only Newton's 3rd law for its solution, also loads strongly on FC2. It is also difficult to understand why item 17 (force in elevator) and item 20 (blocks moving at different speeds) form Factor FC1. It is unclear if correlations through the overall difficulty of the item could explain some of the unexpected structure. 


\subsection{Results - Confirmatory Analyses}

The exploratory analysis of the previous section failed to extract a factor structure that was understandable within a theoretical model of Newtonian mechanics (Table 3.3). For over 50 years, social scientists have argued that research shouldn't rely purely on exploratory techniques but rather that having a robust theoretical framework is paramount to the determination of model validity [106]. According to Cronbach and Meele, there is no validity without an articulated theory and it is, therefore, inappropriate to use only exploratory techniques, such as EFA, on an instrument. Furthermore, EFA results provide

only information about the data itself and should not be construed as providing genuine answers or solutions without a theoretical core [107]. Exploratory methods generally identify some structure, and without a framework, that structure may simply be the result of random fluctuations in the data.

Confirmatory analysis instead proceeds from previously articulated theoretical model and explores how that model can be used to understand the data. Often confirmatory analysis starts with fitting the full theoretical model and then examines a small number of theoretically motivated modifications to the model. The theoretical model of Newtonian mechanics presented in Table 3.3 was used as the starting point for a confirmatory analysis of the FCI. MIRT allows the exploration of this model by constraining the MIRT parameter matrix to the model. This is analogous to a Confirmatory Factor Analysis (CFA) where the analysis proceeds from theoretical model and determines how well the data fit the model. Constrained MIRT is not fully equivalent to CFA because they proceed from different underlying statistical models, but the method of exploring related models is equivalent. 
Table 3.5: Hierarchical MIRT modeling. The $\chi^{2}$ test determines whether the models are statistically different; if so, it measures the improvement of the superior model over the inferior model.

\begin{tabular}{|c|c|c|c|c|c|c|}
\hline $\begin{array}{c}\text { Transformed } \\
\text { Model }\end{array}$ & Transformation & $\begin{array}{c}\text { Original } \\
\text { Model }\end{array}$ & AIC & $\mathrm{BIC}$ & $\begin{array}{c}\text { Chi-Squared } \\
\text { Test }\end{array}$ & $\begin{array}{c}\text { Superior } \\
\text { Model }\end{array}$ \\
\hline 1 & & & 91,067 & 91,668 & & \\
\hline 2 & Remove all lemmas. & 1 & 90,943 & 91,518 & $\chi^{2}(4)=116, p<0.001$ & 2 \\
\hline 3 & Remove RS1. & 2 & 90,920 & 91,488 & $\chi^{2}(1)=21, p<0.001$ & 3 \\
\hline 4 & Combine DF3 with L2. & 3 & 90,942 & 91,510 & & 3 \\
\hline 5 & Combine L1 with L2. & 3 & 90,929 & 91,491 & $\chi^{2}(1)=11, p=0.001$ & 3 \\
\hline 6 & Combine C3 with R1; C4 with R2. & 3 & 90,991 & 91,553 & $\chi^{2}(1)=73, p<0.001$ & 3 \\
\hline 7 & Remove F2. & 3 & 90,941 & 91,490 & $\chi^{2}(3)=27, p<0.001$ & 3 \\
\hline 8 & Remove F5. & 3 & 90,944 & 91,499 & $\chi^{2}(2)=28, p<0.001$ & 3 \\
\hline 9 & Remove F6. & 3 & 90,929 & 91,491 & $\chi^{2}(1)=11, p=0.001$ & 3 \\
\hline 10 & Replace L1 with L2 and DF2. & 3 & 90,933 & 91,521 & $\chi^{2}(3)=7.5, p=0.058$ & 3 \\
\hline
\end{tabular}

Nested models were compared using a likelihood ratio test. If two models with likelihood functions $L_{1}$ and $L_{2}$ differ by $k$ parameters where model 1 has fewer parameters, then the test statistic $\chi^{2}=-2 \ln \left(L_{1} / L_{2}\right)$ has a chi-squared distribution with $k$ degrees of freedom and can be used to test whether the models are significantly different [108].

Bootstrapped means and standard deviations were calculated for the MIRT parameters $\boldsymbol{a}_{j}$ and $d_{j}$. Bootstrapping generates many subsamples of the data with replacement and runs the desired statistical test on each subsample to generate a normal distribution of fit parameters from which the mean value is calculated. For this work, 200 subsamples were generated requiring one week on a modern personal computer.

\subsubsection{Constrained MIRT}

MIRT allows the exploration of student thinking about Newtonian mechanics by constraining the parameter matrix to a model. The $\mathbf{a}_{j}$ parameter matrix can be constrained so that parameters that should not theoretically affect a factor are zero. For example, if the model of Newtonian mechanics in Table 3.3 was used as the basis for a constrained MIRT model, 
then the factor representing DF1, $a_{D F 1}$, could be constrained to be zero except for items 19 and 20. For this analysis, only the first problem in a problem block was retained as before; groups of similar problems $\{5,18\},\{6,7\},\{17,25\}$, and $\{4,15,28\}$ were also retained. Because constrained MIRT is not exploratory, the correlations of these items will not unduly influence the analysis. The 20-item problem set analyzed in this section was then: $1,2,3$, $4,5,7,8,12,13,14,15,17,18,19,20,21,25,28,29$, and 30 .

The starting model for the confirmatory analysis included all the principles introduced in Table 3.3 which were not eliminated by removing blocked items. F7 and C5 were eliminated when blocked items were removed. The FCI has strong internal consistency and most items are positively correlated. To separate a general facility with Newtonian mechanics from a specific facility with one of the principles, an additional factor was added that loaded on all items. The fit statistics of this model, model 1, are shown in Table 3.5. Fit statistics in Table 3.5 apply to the Transformed Model number, as such, model 1 is Transformed Model 1. Model fit can be examined by the amount AIC and BIC changes between models and ultimately from bootstrapping which will show most parameters in the best fitting model have standard deviations that suggest the parameters are significantly different from zero.

After the full model is fit, confirmatory analysis examines theoretically motivated simplifications of the full model. Each transformation in Table 3.5 modified the original model to the transformed model. A likelihood ratio test determined whether the models were statistically different. Model 4 did not change the number of degrees of freedom from model 3; therefore, a chi-squared test could not be performed; however, AIC and BIC could be compared. Some transformations removed a principle from a previous model; other 
transformations combined two principles. For example, in model 5 all items that loaded on either L1 or L2 were set to load on only L2. These models do not exhaust the set of available models, but represented a set of models where a theoretical case could be made for each change.

Model 2 tested a fundamental question about the granularity of student knowledge of the FCI. The set of possible supplementary principles (reasoning and lemmas) is quite large while the set more general core principles (laws, facts, definitions, corollaries, and results) are substantially smaller. Each lemma represented a qualitative interpretation or a special case of a core principle. To determine if the lemmas were important to the understanding of the pattern of answers, model 2 was constructed which removed all lemmas and replaced them with the core principle from which they were derived. Model 2 was a significant improvement over model 1 with very strong changes in AIC and BIC and, therefore, the answering pattern for this sample could be understood without the lemmas. Student thinking about the FCI is better understood in terms of a short list of core principles rather than the extensive lists of qualitative lemmas derived from the core principles. This provides important insight into the number of principles needed to understand student Newtonian thinking while also substantially simplifying the research effort. The model without the lemmas could have been produced by any physics graduate student and should be much less dependent on the experts providing the solutions.

Confirmatory exploration continued by testing a sequence of models either motivated by questions that arose about what part of the core principles the FCI measured or questions about relations between the core principles. For each step, the difference in AIC and BIC between the better fitting model and the less well fitting model are reported. Model 3 
removed the crossing reasoning step RS1 from model 2; this improved model fit (very strong change in AIC, strong change in BIC). RS1 was used only in a subset of expert responses; other experts simply observed that two of the interval lengths were comparable. Model 4 explored whether the vector addition of forces could be viewed as a part of Newton's 2nd law by combining L2 and DF3; model 3 was a significant improvement over model 4 (very strong change in AIC and BIC). These students answer Newton's 2nd law questions and addition of forces questions with different facility. Combining Newton's 1st law (L1) and Newton's 2nd law (L2) to form model 5 from model 3 also did not improve model fit over model 3 (strong change in AIC, very strong change in BIC). A second model that eliminated Newton's 1st law from model 3, model 10, replaced L1 with L2 (Newton's 2nd law) and DF2 (the definition of acceleration). This model was not statistically superior to model 3 and the model increased both AIC (very strong) and BIC (very strong). As such, L1 was retained as a separate entity. Combining $\mathrm{C} 3$ and $\mathrm{C} 4$ representing one-dimensional kinematics into $\mathrm{R} 1$ and $\mathrm{R} 2$ representing three-dimensional kinematics to form model 6 did not improve model fit over model 3 (very strong change in AIC and BIC). Fact F2 (there is not necessarily a force in the direction of motion) addresses a common misconception; removing F2 from model 3 to form model 7 did not improve model fit (very strong change in AIC and BIC). Finally, facts F5 (air resistance is negligible) and F6 (gravity is approximately constant) are additional pieces of information about mechanics; however, their use was only required to eliminate distractors and they were not used by some experts who solved the problem without considering the distractors. Neither model 8 which eliminated F5 from model 3 (very strong change in AIC and BIC) nor model 9 (strong change in AIC, very strong change in BIC) which eliminated F6 from model 3 improved model fit. As such, model 3 which contains only core principles, 
of the item. To allow comparison with the more intuitive 2PL model, an effective difficulty, $b_{j}$, is calculated $b_{j}=-d_{j} / a_{0 j}$. The larger $b_{j}$ the lower the probability the students will answer the item correctly; the 2PL probability function is shown in Eqn. 3.1. The "mirt" package does not report normalized latent variables; the standard deviation of the each latent variable has been absorbed into the $a_{j}$ coefficient. Therefore, the $a_{j}$ coefficient represents the change in log odds if the latent trait increases by one standard deviation.

Table 3.7 presents the discrimination of each principle on each FCI item as well as the standard deviation of each item. For example, the discrimination of FCI item 4 on Newton's 3rd law (L3) is $2.37 \pm 0.29$; a higher discrimination than the other Newton's 3rd law items. The analysis also allows the determination of the relative discrimination of items that test multiple principles. For example, item 21 provides much better discrimination of student knowledge of three-dimensional motion (R1) than Newton's 2nd law (L2). As such, Table 3.7 provides an exceptionally detailed model of what each FCI item measures.

Some alternate forms of the constrained analysis were also performed. The optimal model in Table 3.7 included one factor that loaded on all problems; a factor capturing a student's overall facility with conceptual Newtonian mechanics. The model with this factor $(\mathrm{AIC}=90,920, \mathrm{BIC}=91,488)$ was a significant improvement over the model without this overall factor $(\mathrm{AIC}=94,442, \mathrm{BIC}=94,881)\left[\chi^{2}(20)=3562, p<0.001\right]$ with a very strong change in AIC and BIC. The model with this factor also had superior behavior in tests that compared model 3 to models where additional principles that damaged the model had been introduced. For example, the addition of L3 (Newton's 3rd law) to item 1 produced a significantly less well fitting model with the overall factor, but not without it. The model without this overall factor is presented in the Supplemental Material [109]. 
Table 3.7: Optimal MIRT model 3. The number in parenthesis is the discrimination, $a_{j k}$, for the principle on the item. $a_{0}$ is the discrimination for a factor loaded on all items and $b$ is the difficulty of the item.

\begin{tabular}{|c|c|c|c|c|c|c|}
\hline FCI\# & \multicolumn{4}{|c|}{ Principles } & $a_{0}$ & $b$ \\
\hline 1 & & $\mathrm{C} 3(1.04 \pm 0.30)$ & $\mathrm{L} 4(-0.30 \pm 0.19)$ & $\mathrm{F} 5(0.09 \pm 0.11)$ & $2.25 \pm 0.44$ & $-3.30 \pm 0.22$ \\
\hline 2 & & $\mathrm{R} 1(0.06 \pm 0.05)$ & $\mathrm{C} 3(0.48 \pm 0.12)$ & $\mathrm{L} 4(0.09 \pm 0.05)$ & $1.05 \pm 0.07$ & $-0.86 \pm 0.06$ \\
\hline 3 & $\mathrm{R} 2(0.13 \pm 0.09)$ & $\mathrm{L} 4(0.02 \pm 0.12)$ & $\mathrm{F} 3(0.14 \pm 0.09)$ & $\mathrm{F} 5(0.13 \pm 0.09)$ & $1.65 \pm 0.19$ & $-2.50 \pm 0.12$ \\
\hline 4 & & & $\mathrm{~L} 3(2.37 \pm 0.29)$ & & $1.88 \pm 0.19$ & $-0.72 \pm 0.05$ \\
\hline 5 & & $\mathrm{C} 2(0.64 \pm 0.15)$ & $\mathrm{L} 2(0.51 \pm 0.10)$ & $\mathrm{L} 4(0.50 \pm 0.11)$ & $1.49 \pm 0.13$ & $-0.38 \pm 0.05$ \\
\hline 7 & & $\mathrm{C} 1(0.16$ & $\pm 0.09) \quad \mathrm{L} 1(0.01$ & $\pm 0.05)$ & $0.64 \pm 0.06$ & $-3.42 \pm 0.26$ \\
\hline 8 & & $\mathrm{~L} 1(-0.27=$ & $\pm 0.08)$ & $30 \pm 0.09)$ & $1.41 \pm 0.12$ & $-2.18 \pm 0.09$ \\
\hline 12 & & $\mathrm{R} 1(0.55$ & $\pm 0.08)$ & $\pm 0.07)$ & $0.75 \pm 0.07$ & $-3.73 \pm 0.31$ \\
\hline 13 & & $\mathrm{~L} 4(0.29$ & $\pm 0.10) \quad \mathrm{F} 2(0.27$ & $\pm 0.09)$ & $2.36 \pm 0.17$ & $-1.34 \pm 0.05$ \\
\hline 14 & & $\mathrm{DF} 1(0.22 \pm 0.08)$ & $\mathrm{R} 1(1.03 \pm 0.15)$ & $\mathrm{L} 4(0.31 \pm 0.08)$ & $0.78 \pm 0.07$ & $-0.99 \pm 0.09$ \\
\hline 15 & & & $\mathrm{~L} 3(0.79 \pm 0.05)$ & & $0.87 \pm 0.06$ & $-0.78 \pm 0.07$ \\
\hline 17 & & DF3(0.70 \pm 0.13$)$ & $\mathrm{L} 1(0.60 \pm 0.12)$ & $\mathrm{L} 4(0.14 \pm 0.06)$ & $1.64 \pm 0.13$ & $-0.18 \pm 0.04$ \\
\hline 18 & $\mathrm{C} 2(0.65=$ & $\pm 0.14) \quad \mathrm{L} 2(0.58$ & $3 \pm 0.11) \quad$ L4 $(0.50$ & $\pm 0.11) \quad \mathrm{F} 2(0.27 \pm 0.09)$ & $1.71 \pm 0.13$ & $-0.31 \pm 0.04$ \\
\hline 19 & & & $\mathrm{DF} 1(0.16 \pm 0.08)$ & & $1.28 \pm 0.08$ & $-2.04 \pm 0.09$ \\
\hline 20 & & DF1(0.44 & $\pm 0.12) \quad$ DF $2(0.2$ & $23 \pm 0.10)$ & $1.12 \pm 0.09$ & $-0.83 \pm 0.05$ \\
\hline 21 & & $\mathrm{R} 1(0.82$ & $\pm 0.10)$ & $\pm 0.07)$ & $0.80 \pm 0.07$ & $0.62 \pm 0.07$ \\
\hline 25 & & DF3 $(0.70$ & $0 \pm 0.13)$ & $0 \pm 0.13)$ & $1.91 \pm 0.16$ & $-0.13 \pm 0.03$ \\
\hline 28 & & & $\mathrm{~L} 3(1.28 \pm 0.09)$ & & $1.70 \pm 0.09$ & $-0.98 \pm 0.05$ \\
\hline 29 & & $\mathrm{~L} 4(-0.10 \pm 0.09)$ & $\mathrm{F} 1(0.09 \pm 0.06)$ & $\mathrm{F} 3(0.09 \pm 0.06)$ & $0.17 \pm 0.06$ & $-12.12 \pm 6.28$ \\
\hline 30 & & $\mathrm{~L} 4(0.29 \pm 0.09)$ & $\mathrm{F} 2(0.19 \pm 0.07)$ & $\mathrm{F} 4(0.24 \pm 0.10)$ & $1.11 \pm 0.08$ & $-0.57 \pm 0.05$ \\
\hline
\end{tabular}

MIRT can also be used to estimate the ability of each student to answer each item. The correlations of these abilities are presented in Fig. 3.5. Because one factor was loaded onto all items, these abilities represent that difference between the student's general ability to solve a conceptual mechanics question and his or her ability to apply a specific principle. For students with a fully developed expert understanding of mechanics, we would expect their ability to apply each principle to be equal, and therefore their difference in ability to be zero. Fig. 3.5 shows multiple principles with large correlations and large differences in the strength of the correlation between different items. From this diagram, we can infer that the students studied have differing but correlated abilities with concepts of velocity and acceleration (DF1, DF2), with Newton's 1st law (L1) and the addition of forces (DF3), and with Newton's 2nd law (L2) and the law of gravitation (L4). Additional instruction may be required to allow students to fully integrate these concepts. MIRT, then, may also represent a 


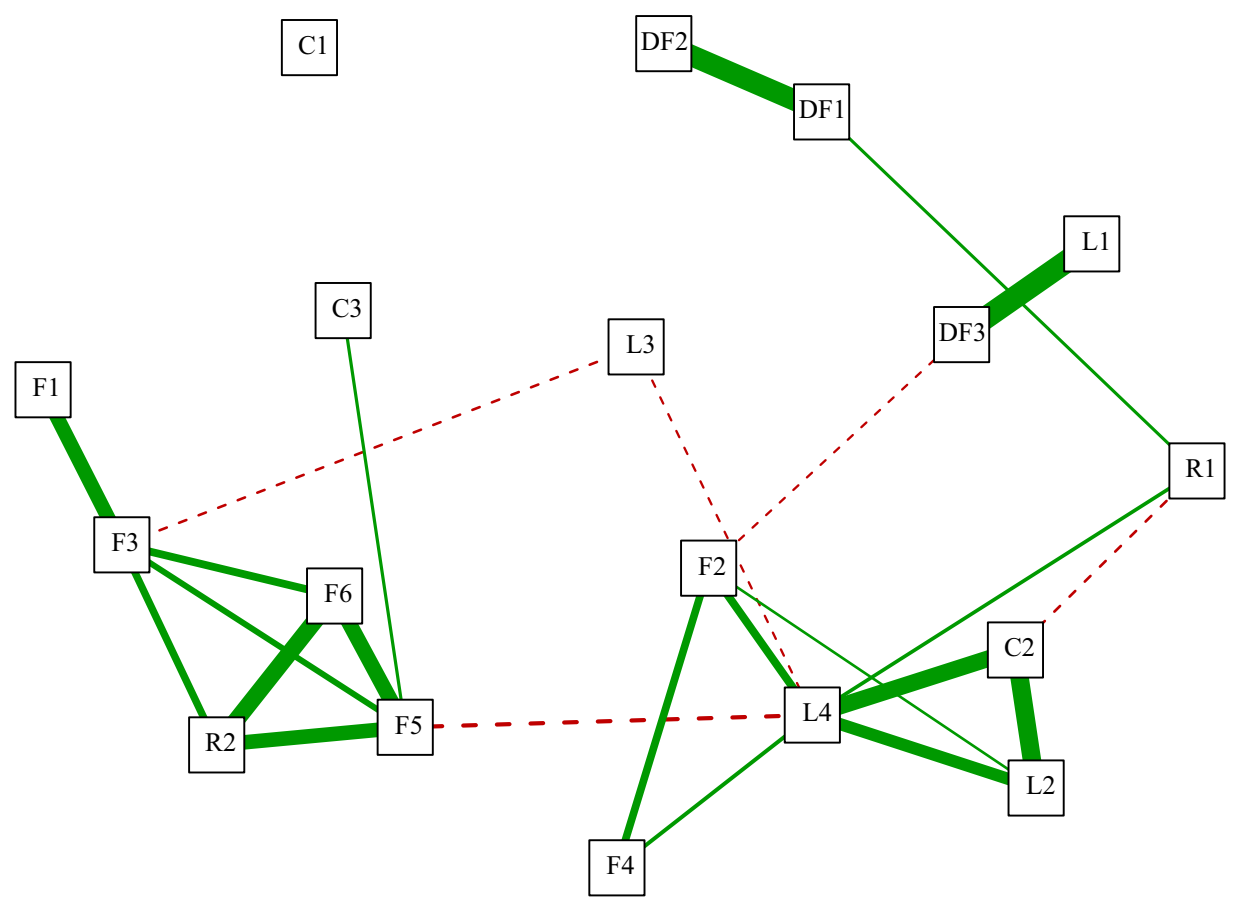

Figure 3.5: Correlation matrix of student ability using model 3. Lines represent correlations with $|r|>0.15$. Line thickness represents the size of the correlation. Solid (green) lines represent positive correlations; dashed (red) lines negative correlations.

tool which can be used to probe the structure of knowledge and to quantitatively characterize expert/novice differences and to localize where additional integration of knowledge is needed.

\subsubsection{Comparison with Original FCI Model}

The FCI authors suggested a detailed structure for the FCI dividing the test into 6 general categories and 23 fine-grained principles (see Table I in [13]). The fine-grained principles play the same role as the principles in the theoretical model in Table 3.3. The FCI was revised in 1995; the revised test included 3 new problems which were not categorized. These items, revised FCI items 5, 18, and 30, will not be included in this analysis.

Fitting a model implementing the structure suggested in the original FCI paper on 
the set of items $1,2,3,4,7,8,12,13,14,15,17,19,20,21,25,28$, and 29 from the revised $\mathrm{FCI}$ produced a model with $\mathrm{AIC}=75,260$ and $\mathrm{BIC}=75,453$. Using the constrained MIRT model of the previous section on the more restricted problem set produced a model with substantially better model fit $[\mathrm{AIC}=74,812 ; \mathrm{BIC}=75,277]$, a very strong change in $\mathrm{AIC}$ and BIC. A likelihood ratio test showed that the constrained MIRT model had significantly better model fit $\left[\chi^{2}(13)=474, p<0.001\right]$. As such, while the model proposed by the FCI authors captured their motivation as the creators of the instrument, model 3 produced a better fit for this student population.

\subsection{Discussion}

This Chapter investigated five research questions; they will be discussed in the order proposed.

RQ1: What factor structure is extracted for the FCI by MIRT? Is this structure consistent with the results of other factor analyses? MIRT identified a 9-factor solution as optimal for the full 30-item FCI. Other studies have identified 5-factor [47] and 6-factor [48] post-test models as optimal. It is possible that the larger sample used in the present study combined with the strong incentives given for correctly answering the items (the FCI score was counted toward the student's grade) allowed this study to resolve more detailed structure in the FCI. The 9-factor model, while the best statistically based on likelihood ratio tests, was not the best model on all fit statistics (Table 3.2). The fit statistics could also support the identification of either a 5-factor or 6-factor model. All three of these studies identified more factors than Huffman and Heller [46]; however, this may have resulted from 
the differing size and quality of the samples as well as the different criteria used to select the optimal number of factors.

The factors extracted can also be compared. If the 9-factor solution found in this study resulted because of superior resolution of the factors, we would expect some of the factors in the previously reported models to split to form the additional factors in this study. Some commonality can be found between the 5-factor [47], 6-factor [48], and our 9-factor model. The groups of physically similar items $\{5,18\},\{6,7\},\{17,25\}$, and $\{4,15,16,28\}$ do factor together in all models, except that item 16 often does not factor with the Newton's 3rd law group. The 5-factor model shows the same tendency of blocked items to factor together that we saw in the 9 -factor model; this effect was less pronounced in the 6 -factor model. All the factor models are difficult to support in terms of the actual structure of the physical principles needed to solve the problems shown in Table 3.3. As such, it is difficult to support the proposition that EFA is providing fundamental insights into the knowledge structure of physics students as measured by the FCI.

RQ2: Can parts of this factor structure be explained by factors other than the structure of student knowledge of Newtonian mechanics?

Correlation analysis identified two non-physical sources of relations between FCI items which could affect the factor structures: correlations through the blocking of items into groups and correlations through total score. The effect of blocking was clear in Table 3.1 with most blocked questions sharing the same factor with the exception of items 5 and 6 . The strong correlation of many blocked items can also be seen in the overall correlation matrix (Fig. 3.2). Further analysis retained only the first item in each group; the nonphysical correlations created by blocking could not be corrected statistically. While the 
possible correlation of blocked items seems relatively uncontroversial, we know of no previous research that identifies it as a possible source of a non-physical perturbation on the factor structure or other analysis. The possible correlation between total score could be deduced through the studies showing the FCI as a very internally consistent instrument $[18,19]$ as well as Huffman and Heller's identification of the FCI as a single-factor instrument [46]. This internal consistency is clearly demonstrated in Fig. 3.2 showing all correlations are positive. The possibility of the difficulty of an item impacting the factor structure was discussed briefly by Scott, Schumayer, and Gray [47].

The correlations through overall test score were removed by calculating a partial correlation matrix (Fig. 3.3) which continued to show the effect of problem blocking and revealed a third source of correlation. There were four groups of items in the FCI which are answerable using very similar physical principles. One group, items requiring Newton's 3rd law for their solution, was expected. This group forms one of the factors in each published analysis [47, 48, 64] except Huffman and Heller [46]. The other three groups do not seem to represent special combinations of reasoning particularly important to understanding mechanics and the repetition of these principles seems likely to be accidental. These groups $\{5,18\},\{6,7\}$, and $\{17,25\}$ had large factor loadings in the same factor in all published models. It seems likely that the repetition of these blocks artificially influenced the factor structure; many other equally important combinations of physical reasoning could have been repeated.

RQ3: If blocked items and repeated reasoning groups are removed, is the resulting factor structure consistent with Newtonian mechanics? An EFA was also presented for a reduced set of FCI items which removed all but the first item in each problem block and removed 
the second item of the $\{5,18\}$ and $\{17,25\}$ groups and the first item of the $\{6,7\}$ group. This EFA found a 6-factor solution (Table 3.4); however, the factors make little physical sense. Factor 1 mixed a Newton's 1st law problem involving an elevator with the analysis of two plots with zero acceleration. Factor 2 contains a mixture of items including Newton's 3rd law, one-dimensional constant acceleration, and a position vs. time plot involving objects with constant velocity and acceleration. Factor 3 includes three Newton's 3rd law items but also two-dimensional zero acceleration motion and one-dimensional motion under gravity. As such, factor analysis, once non-physical or accidental correlations are removed, does not extract a factor structure consistent with Newtonian mechanics. As the designers intended, the FCI is a single-factor instrument [60]. The reason for the coherence can be seen in Fig. 3.4 where many items test multiple general domains.

RQ4: Can theoretically constrained MIRT produce a model of the physical constructs measured by the FCI? If so, what is the optimal model of the FCI for this student population?

Constrained MIRT allowed a confirmatory exploration of a set of related models grounded in the traditional theoretical framework of Newtonian mechanics. This exploration showed, while expert solutions to the FCI were cast in a number of lemmas which converted the mathematical framework of mechanics to language-based principles, that these were not needed to understand the structure of student understanding. This implies student thinking can be productively understood by a set of core principles grounded in the model of Table 3.6.

The optimal model 3 supported the differentiation in student thought between Newton's 1st law and Newton's 2nd law as well as the difference between one-dimensional and threedimensional constant acceleration kinematics. Facility with the vector addition of forces was 
also shown to be distinct from facility with Newton's 2nd law.

Table 3.7 shows the optimal MIRT model 3. The number in parenthesis next to the principle label is the discrimination for the principle. Because an overall factor loading on all items was included, $a_{0}$, the discrimination, $a_{j>0}$, of the individual principles represents the additional effect of the specific ability over the student's general ability with Newtonian mechanics. Some of the discrimination parameters are very small indicating that the item does not require additional facility with the principle over the student's general ability to answer FCI questions correctly. Some discriminations are negative which may be a sign of a problematic item. Items with only one strongly discriminating principle might be claimed to be good marker items for the skill represented by the principle. Items 1, 2, 12,14 , and 21 require multiple principles but discriminate on one principle more strongly than the others. These questions might be used to characterize students' knowledge on the high discrimination principle. Items 4, 15, 19, and 28 require only one principle, and therefore could be used as a measure ability to perform this principle; however, three of the four represent Newton's 3rd law. Items 5, 17, 18, and 25 require multiple principles with commensurate and large discriminations. These items measure multiple abilities at the same time, but do not differentiate between the abilities. Finally, a number of items have small discrimination values for all principles: items $3,7,13,29$, and 30 . These items do not contribute additional information about specific abilities. Item 8 had negative discrimination; this may indicate the item is not functioning correctly.

MIRT provides a new lens for examining physics evaluations. If this lens proves valuable, it will suggest certain desirable properties in future evaluations. First, the structure and number of items should allow non-compensatory MIRT models to be fit to extract 
item/principle-level difficulty parameters. Second, each item should provide additional information about some ability. Third, the instrument should be invertible so that a linear combination of the scores on a subset of items provides an estimate of the ability for a each principle, giving practitioners a detailed characterization of their learning outcomes.

RQ5: Does the structure proposed by the FCI's authors provide a superior description of the instrument to the optimal model identified by MIRT? The structure suggested by the authors of the FCI [13] was also fit to the data set and the result compared to the optimal model 3 identified by MIRT. Model 3 outperformed the model suggested with the publication of the FCI. Part of the reason the published structure has not been recovered may be that other models fit the FCI better. This seems unlikely to be the primary reason for the mismatch between the proposed model and model 3. Table 3.7 and Fig. 3.4 as well as Hestenes and Halloun's insistence that the FCI measures a single Newtonian force concept [60] show that the instrument simply was not constructed to factor well. There are very few items that use a single principle and only Newton's 3rd law, not Newton's 1st or 2nd law, is used independently and is repeated multiple times in the unblocked model (Table 3.7). Most FCI items measure multiple physical principles at once.

This work identified the blocking of items in the FCI as a source of correlations not related to the student's ability to answer conceptual physics questions. To eliminate these correlations, only the first item in a problem block should be retained; as such, items 6, 9, $10,11,16,22,23,24,26$, and 27 were removed from the FCI producing a 20-item version of the FCI. The model in Table 3.6 can be used to understand the effect of this reduction. The blocked items to be removed are shown in bold in both Table 3.6 and Fig. 3.4. Removing these items eliminated principles $\mathrm{F} 7$ and $\mathrm{C} 5$ while reducing coverage of $\mathrm{R} 2$ and $\mathrm{C} 4$. In 
general, these reductions still leave the coverage of the FCI intact except for the elimination of an explicit use of friction.

Traxler et al. [19] also suggested a reduced 19-item instrument (including FCI questions: $1,2,3,4,5,7,8,10,11,13,16,17,18,19,20,25,26,28$, and 30) to remove items with reliability problems and to remove items unfair to either men or women. The items removed to produce the 19-item instrument are underlined in Table 3.6 and Fig. 3.4. While this reduction removes seven items from both kinematics and dynamics in Table 3.6, the coverage of kinematics required more principles than that of dynamics. The removal of unfair items from R1, R2, and C4 substantially changes the coverage of the instrument. Removing both blocked and unfair items further reduces the coverage.

To produce a fair instrument while maintaining coverage, it may be necessary to retain some blocked and unfair items but to balance the degree and number of unfair items for both men and women. Traxler et al. [19] reported that two of the removed items were unfair to men, items 9 and 15. If these two items are retained as well as items 14 and 27, which were unfair to women with similar Differential Item Functioning statistics, the overall score should be gender fair. Blocked items 11 and 26 could also be retained to maintain coverage. Retaining these items would increase coverage of some kinematic principles while providing coverage of F7 and C5. This would leave a reduced 21-item FCI instrument containing: items 1, 2, 3, 4, 5, 7, 8, 9, 11, 13, 14, 15, 17, 18, 19, 20, 25, 26, 27, 28, and 30. Blocked items 10 and 16 were removed because there was sufficient coverage of the principles required for their solution. 


\subsection{Limitations}

This work was performed with a single sample drawn from a single institution. Additional studies are necessary to determine if the conclusions are general. The sample was analyzed in aggregate; additional analysis is needed to determine if the results apply to all student sub-groups. The analysis did not consider the role of misconceptions; an extended theoretical model including misconceptions should also be tested.

This work began with a model constructed from a sample of expert solutions at a single institution. Alternate models certainly can and should be constructed; MIRT provides the tool needed to determine which model best fits student thinking. The model presented in this work should not be considered the end point, but the beginning of a more detailed exploration of conceptual Newtonian mechanics that will take many years to complete.

\subsection{Implications}

This worked showed a theoretical model of introductory mechanics could be useful in understanding the results of conceptual inventories. Such models can be constructed for other conceptual areas of physics and could form a basic tool for understanding the detailed results of PER instructional innovations. The constrained MIRT analysis technique allowed the fine-grained exploration of the constructs measured by the FCI and may be a powerful tool for improving our understanding of student knowledge. EFA did not produce a factor structure that was useful in understanding the FCI and it is likely that purely exploratory tools may not yield generalizable results. Part of the reason for the failure of EFA was correlations produced by the blocks of questions in the FCI. The practice of using blocks of 
questions with the same stem may make PER instruments difficult to interpret statistically and should be discontinued.

This work showed that when all items identified as problematic because of correlations produced by blocking and all items identified as unfair or unreliable by Traxler et al. [19] were removed, then the coverage of kinematics of the modified FCI is reduced. We instead proposed a 21-item reduced FCI to maintain coverage while balancing unfair items; to have to decide between coverage and fairness is unacceptable. While this 21-item instrument could be used for the near future, the identification of unfair items and blocked items as problematic in addition to the lack of coherent sub-scales suggest that it is time to revisit the construction of the FCI and to modernize it to remove some of the difficulties identified in recent research.

\subsection{Conclusions}

The exploratory analysis of the FCI identified a 9-factor solution which showed some similarities to previously published solutions. Further analysis showed many of the factors in the 9-factor solution, and the previously published solutions, could have resulted from the use of multiple problem blocks and the repetition of physically similar items. Exploratory factor analysis was repeated removing these correlated items; the resulting 6-factor solution could not be reconciled with the theoretical structure of Newtonian mechanics. Constrained MIRT was then employed to determine the optimal model of the FCI for the student population studied within the framework of a theoretical model. The optimal model contained only core

principles of mechanics and did not contain subsidiary principles derived from these core 
principles. The optimal model differentiated between Newton's 1st and 2nd law; between Newton's 2nd law and the principle of vector addition of forces; and between one-dimensional and three-dimensional kinematics. The optimal model identified by MIRT was substantially statistically superior to the original model proposed by the authors of the FCI. 


\section{Chapter 4}

Evaluation of the Knowledge Structure Measured by

Conceptual Survey of Electricity and Magnetism 
This chapter replicates the previous study on the FCI using data sets from two different institutions to explore the structure of the Conceptual Survey of Electricity and Magnetism (CSEM) to determine whether MIRT results can be considered general. If so, the structure of knowledge found using MIRT from on sample could be used to understand the knowledge structure of students in other samples at other schools without having to repeat the MIRT modeling process. The previous chapter showed that MIRT models could be constrained to a theoretical model of Newtonian mechanics and used to explore theoretically motivated modifications of the model. Therefore, a theoretical model of the CSEM expert solution structure was constructed and tested using MIRT as a confirmatory analysis. This model was then transformed with theoretically justifiable modifications until a best-fitting model was found.

The CSEM was introduced nearly 20 years ago and has become one of the most used conceptual instruments for understanding conceptual knowledge of electricity and magnetism [16]. The CSEM was developed following the success of the FCI [13] in demonstrating the ineffectiveness of traditional instruction in fostering conceptual learning [14]. Like the FCI, the CSEM was developed to test student misconceptions as well as their physics knowledge. The authors further intended the instrument to serve as a broad summary of student learning in electricity and magnetism rather than a granular measure of student understanding [16].

\subsection{Reliability and Validity}

The structure, reliability, and validity of PER conceptual instruments is an active area of research; however, most of this research has focused on the FCI. Few studies have analyzed 
the item-level validity, reliability, or fairness of the CSEM.

Classical Test Theory (CTT) provides methods to examine item validity though the calculation of difficulty and discrimination. The difficulty of an item is defined as its average score; a higher difficulty score indicates an easier item and a lower difficulty score a harder item. The discrimination is defined as the difference in the average score of the highest performing students and the lowest performing students. Items with either very high or very low difficulty or low discrimination are "problematic" and present validity threats to the instrument $[59,110]$

Maloney et al. reported CSEM item-level difficulty for both algebra-based and calculusbased introductory electricity and magnetism courses [16]. Their study found four problematic items with item 3 too easy for calculus-based students (difficulty above 0.8), items 14 too difficult for both calculus and algebra-based students (difficulty below 0.2), and items 20 and 31 too difficult for algebra-based students. Maloney et al. used principle component analysis to measure the factor structure of the CSEM and found an optimal 11-factor model of the instrument. This model was discarded as containing too many factors with too little variance explained by each. The 11-factor model structure was not reported. Planinic identified six conceptual areas measured by the CSEM in a study comparing Croatian students to American students: electric charge and force (items 1-3, 5, 6, and 8), electric field and force (items 9 and 12-15), electric potential and energy (items 11 and 16-20), magnetic field and force (items 21-23, 25, 26, and 28), electromagnetic induction (items 29-32), and Newton's laws (items 4, 7, 10, 24, and 27). The conceptual areas were identified qualitatively by grouping the 11 conceptual areas identified by Maloney et al. [16] to produce groups of items sufficiently large for analysis. The difficulty of the items in each conceptual area was 
calculated finding similar results for both populations [111].

Other studies have investigated only a subset of the items in the CSEM. Meltzer investigated items 18 and 20 to explore changes between pretest and post-test responses regarding the intersection of electric field and potential concepts [112]. Leppävirta investigated CSEM items that probed Newton's 3rd law (items 4, 5, 7, and 24) showing that $20 \%$ of students had an alternative model of Newton's 3rd law on the pretest which was reduced to $10 \%$ on the post-test [113].

Gender differences in performance on the CSEM have also been investigated. Kohl and Kuo [114] examined the difference in the gender gap on the CSEM before and after switching to Studio Physics, finding the course transition reduced the gap in normalized gain. Studio physics is an instructional model that integrates short lectures with group work and hands-on activities in a classroom where students are grouped around tables [115]. Kreutzer and Boudreaux [116] also measured a difference in performance by gender in the CSEM. Pedagogical changes such as "affirming domain belongingness in women" greatly reduced the gap. No research exists on differences by other demographic characteristics.

\subsection{Factor Analysis}

Extensive work on the factor structure of PER conceptual instruments has been performed; however, the majority of this work has investigated the FCI [46, 60, 61, 47, 64, 48, 45] and the Force and Motion Conceptual Evaluation (FMCE) [15, 62].

Beyond the initial factor analysis conducted by Maloney et al. [16] when constructing the CSEM, additional work exploring the factor structure of the instrument has not been 
reported.

\subsection{Item Response Theory}

Many studies have employed Item Response Theory (IRT) to probe individual item performance on PER conceptual inventories; however, again, the majority of this research has focused on the FCI. No work replicating IRT results on the CSEM was identified.

\subsection{Research Questions}

This study seeks to answer the following research questions:

RQ1: What is the optimal model of student knowledge measured by the CSEM? Are the principles forming the optimal model consistent across samples?

RQ2: Are the parameters of the optimal models consistent between samples?

\subsection{Sample}

This study will examine two samples drawn from different institutions in the United States.

Sample 1: Sample 1 was drawn from 14 semesters of calculus-based introductory electricity and magnetism courses at a large southern land-grant university serving approximately 25,000 students. The undergraduate population had ACT scores ranging from 23 to 29 (25th to 75th percentile) [93]. The institution held a Carnegie Classification of "Highest Research Activity" for all semesters studied [117]. The overall undergraduate demographics were $77 \%$ White, 8\% Hispanic, 5\% African American, 2\% Asian with other groups each 3\% or less. 
The sample was primarily male (77\%) [93].

For the entire study, the course was comprised of two 50-minute lectures per week with an additional two 2-hour weekly laboratories. The CSEM was given as a laboratory quiz pre- and post-instruction with the student's scores counting toward the course grade. The course was taught and overseen by the same instructor for each of the 14 semesters included in this study. The aggregate data set contains 2014 students who completed the course for a grade and received credit for CSEM pretest and post-test. The data set was also analyzed by Henderson et al. [118] to explore gender fairness.

Sample 2: Sample 2 was drawn from 13 semesters of calculus-based introductory electricity and magnetism courses at a large eastern land-grant university serving approximately 30,000 students. The undergraduate population had ACT scores ranging from 21 to 26 (25th to 75th percentile) [93]. The institution was first rated as "Highest Research Activity" a year prior to the completion of data collection [117]. The overall undergraduate demographics were 79\% White, $6 \%$ International, 5\% African American, 4\% Hispanic, 2\% Asian with other groups each $4 \%$ or less. The sample was primarily male (81\%) [93].

Unlike Sample 1, the instructional environment for Sample 2 was variable. For the first 4 years of the study, the course was taught by 14 separate instructors with standings ranging from full professor to late-career graduate student. For this period, the course was comprised of four 50-minute lectures and a single 2-hour laboratory each week. A Learning Assistant (LA) program was implemented to improve conceptual learning [119]. Undergraduate students who had previously completed the course were hired to work as helper instructors (LAs). The first hour of lab was dedicated to students working on the University of Washington Tutorials in Introductory Physics [120] with the LA serving as the 
lead lab instructor with the assistance of a graduate teaching assistant (TA). For the second half of the laboratory, students performed traditional lab experiments under the instruction of the TA. LAs were required to attend a course in science teaching from an expert from the College of Education and were overseen and further trained by an experienced physics instructor. Once the LA program was discontinued at the end of its funding in 2015, the course was modified to a different structure with three 50-minute lectures per week with one 3-hour weekly lab. After 2015, all courses were team-taught by a pair of experienced instructors. The CSEM grading policy was set by the individual instructors. The aggregated data set contains 2657 students who completed the course for a grade and completed CSEM pretest and CSEM post-test. This data set was also analyzed by Henderson et al. [118].

As with any analysis, it is preferable to have a stable research environment. Theoretically, IRT should be "sample independent" and return the same results regardless of population; however, this assumes all populations receive relatively consistent coverage of the material so that the ordering of items by the IRT difficulty parameter is consistent for all students. The variability in Sample 2 means this assumption is unlikely to hold for this sample and we find that the difficulty parameters are indeed different between samples. It is also likely that this variability influenced the standard deviations of the parameters in Sample 2.

Sample 1 contained $N_{1}=2014$ subjects with an average CSEM post-test percentile score of $63.7 \%$. Sample 2 contained $N_{1}=2657$ subjects with an average CSEM post-test percentile score of $44.7 \%$. 


\subsection{Exploratory Factor Analysis}

MIRT Exploratory Factor Analysis (EFA) was conducted for each sample, testing solutions from 1 to 10 factors. Though the range of factors was selected arbitrarily, a best fitting model was found for each sample where the AIC and BIC were minimized as well as by applying a likelihood ratio test to subsequence models. Because BIC is a more conservative estimate, it will often select a model with fewer factors.

\subsubsection{Sample 1}

A 9-factor model was selected as optimal based on a likelihood ratio test $\left[\chi^{2}(22)=\right.$ 95.37, $p<0.001$ ] comparing the 8-factor and 9-factor models. The 9-factor model minimized AIC and had reasonably good fit with an RMSEA of 0.031 , CFI of 0.969 and TLI of 0.936. BIC selected a much simpler 3-factor model with worse, but still acceptable, fit statistics: RMSEA $=0.033$, CFI 0.940, and TLI 0.930. The fit statistics for models with 1 to 10 factors are presented in Table 4.1. The 9-factor (varimax rotation) solution is shown in Table 4.2. In the optimal 9-factor model, a number of questions fail to load on any factors $(2,15,22$, $24,28,30,32)$ with a cutoff of 0.3 .

\subsubsection{Sample 2}

An 8-factor model was selected as optimal based on a likelihood ratio test $\left[\chi^{2}(25)=\right.$ 183.18, $p<0.001]$ comparing the 8-factor model to the 7 -factor model. The 8-factor model minimized AIC and had very good fit with an RMSEA of 0.020, CFI of 0.989, and TLI of 0.979. BIC selected a much simpler 3-factor model with worse, but still good, fit statistics: 


\begin{tabular}{cccccc} 
Factors & AIC & BIC & RMSEA & TLI & CFI \\
\hline 1 & 71,380 & 71,739 & 0.049 & 0.84 & 0.85 \\
2 & 70,716 & 71,248 & 0.038 & 0.90 & 0.91 \\
3 & 70,435 & 71,136 & 0.033 & 0.93 & 0.94 \\
4 & 70,295 & 71,159 & 0.030 & 0.94 & 0.95 \\
5 & 70,177 & 71,198 & 0.027 & 0.95 & 0.97 \\
6 & 70,099 & 71,271 & 0.036 & 0.91 & 0.94 \\
7 & 70,084 & 71,402 & 0.034 & 0.92 & 0.95 \\
8 & 70,065 & 71,523 & 0.032 & 0.93 & 0.96 \\
9 & 70,017 & 71,610 & 0.031 & 0.94 & 0.97 \\
10 & 70,071 & 71,793 & 0.029 & 0.94 & 0.97 \\
\hline
\end{tabular}

Table 4.1: Exploratory MIRT fit statistics for models with from 1 to 10 factors for Sample 1.

RMSEA of 0.028 , CFI of 0.967 , and TLI of 0.959 . The fit statistics for models with 1 to 10 factors are presented in Table 4.3. The 8-factor model (varimax rotation) is shown in Table 4.4 .

\subsection{Theoretical Framework}

A theoretical model of the knowledge structure measured by the CSEM was developed using multiple expert solutions of the instrument. Instructors in the classes studied and members of the research team were asked to provide detailed solutions to the CSEM. These solutions were decomposed to the sentence level. Sentences expressing the same physical reasoning were grouped together and a general statement of that reasoning, called a principle, was constructed. Table 4.5 presents the list of principles identified. Each principle was classified as a Law (L) representing important physical laws such as Newton's 2nd law, as a Definition (DF) introducing an important new quantity, or as a Fact (F) representing knowledge about the universe that did not rise to the level of a law. From these fundamental pieces of information, Corollaries (C) were derived as important secondary results. The 


\begin{tabular}{|c|c|c|c|c|c|c|c|c|c|c|}
\hline$\#$ & FC1 & FC2 & FC3 & FC4 & FC5 & FC6 & FC7 & FC8 & FC9 & $\bar{d}$ \\
\hline 1 & & & & 0.31 & & & & & & -2.47 \\
\hline 2 & & & & & & & & & & -1.20 \\
\hline 3 & -0.78 & & & & & & & & & -0.95 \\
\hline 4 & -0.86 & & & & & & & 0.31 & & -0.44 \\
\hline 5 & -0.84 & & & & & & & & & -0.08 \\
\hline 6 & -0.32 & & -0.37 & & & & & & & -1.27 \\
\hline 7 & -0.44 & & & & & & & 0.74 & & -0.59 \\
\hline 8 & & & -0.71 & & & & & & & -0.71 \\
\hline 9 & & & -0.70 & & & & & & & -0.98 \\
\hline 10 & -0.34 & & & & & & -0.61 & & & -0.13 \\
\hline 11 & & & & & & & -0.32 & & & 0.93 \\
\hline 12 & & & & & & & -0.36 & & & -2.26 \\
\hline 13 & & & & & & 0.65 & & & & -0.98 \\
\hline 14 & & & & & & 0.60 & & & & 0.79 \\
\hline 15 & & & & & & & & & & -0.65 \\
\hline 16 & & & & & & & & & -0.36 & -0.47 \\
\hline 17 & & & & & & & & & -0.34 & -1.34 \\
\hline 18 & & & & & 0.68 & & & & & -0.69 \\
\hline 19 & & & & & 0.31 & & & & -0.32 & -1.53 \\
\hline 20 & & & & & 0.67 & & & & & 0.33 \\
\hline 21 & & -0.67 & & & & & & & & -0.92 \\
\hline 22 & & & & & & & & & & 0.65 \\
\hline 23 & & & & 0.73 & & & & & & -1.40 \\
\hline 24 & & & & & & & & & & 0.12 \\
\hline 25 & & -0.40 & & & & & & & & -0.39 \\
\hline 26 & & & & 0.71 & & & & & & -1.45 \\
\hline 27 & & -0.54 & & & & & & & & -0.90 \\
\hline 28 & & & & & & & & & & -1.04 \\
\hline 29 & & & & 0.34 & & & & & & 0.07 \\
\hline 30 & & & & & & & & & & -1.10 \\
\hline 31 & & -0.39 & & 0.38 & & & & & & 1.06 \\
\hline 32 & & & & & & & & & & 0.24 \\
\hline
\end{tabular}

Table 4.2: Optimal MIRT Exploratory Factor Analysis model for Sample 1. 


\begin{tabular}{cccccc} 
Factors & AIC & BIC & RMSEA & TLI & CFI \\
\hline 1 & 97,862 & 98,239 & 0.040 & 0.92 & 0.92 \\
2 & 97,249 & 97,808 & 0.032 & 0.95 & 0.95 \\
3 & 96,954 & 97,690 & 0.028 & 0.96 & 0.97 \\
4 & 96,850 & 97,756 & 0.026 & 0.96 & 0.97 \\
5 & 96,765 & 97,836 & 0.022 & 0.97 & 0.98 \\
6 & 96,692 & 97,922 & 0.030 & 0.95 & 0.97 \\
7 & 96,681 & 98,064 & 0.029 & 0.96 & 0.97 \\
8 & 96,548 & 98,078 & 0.020 & 0.98 & 0.99 \\
9 & 96,586 & 98,257 & 0.019 & 0.98 & 0.99 \\
10 & 96,605 & 98,412 & 0.021 & 0.98 & 0.99 \\
\hline
\end{tabular}

Table 4.3: Exploratory MIRT fit statistics for models with from 1 to 10 factors for Sample 2.

expert solutions contained a number of qualitative statements that interpreted the laws, definitions, and corollaries; these were called Lemmas (LM). Table 4.5 shows the classification of the principles into laws, definitions, facts, corollaries, and lemmas as well as the higher order principle from which a lower order principle was derived. As was found for the FCI, expert solutions did not contain all the higher order principles from which the lemmas and corollaries were derived; these higher order principles were inferred and added to the model. Table 4.5 also presents the CSEM items requiring each principle for their solution.

The theoretical model in Table 4.5 differs from the models of Planinic [111] and Maloney et al. [16] because it is grounded in the reasoning found in expert solutions. A course-grained model related to that of Planinic [111] is presented, but has substantially worse model fit than the model described above.

This process was substantially less straightforward for the CSEM than for the FCI. There were two sets of principles which could not be distinguished by the items in the instrument because they all loaded on the same items. A principle will be said to "load" on an item if it is required for the solution of the item following the terminology of factor 


\begin{tabular}{|c|c|c|c|c|c|c|c|c|c|}
\hline$\#$ & FC1 & FC2 & FC3 & FC4 & FC5 & FC6 & FC7 & FC8 & $\mathrm{d}$ \\
\hline 1 & & & & & & & -0.53 & & -0.96 \\
\hline 2 & & & & & & & -0.43 & & 0.59 \\
\hline 3 & & & -0.38 & & & & & 0.46 & -1.13 \\
\hline 4 & & & -0.91 & & & & & & -0.21 \\
\hline 5 & & & -0.81 & & & & & & 0.11 \\
\hline 6 & & & & -0.34 & & 0.44 & & & -0.55 \\
\hline 7 & -0.31 & & -0.64 & & & & & -0.41 & 0.00 \\
\hline 8 & & & & & & 0.72 & & & -0.36 \\
\hline 9 & & & & & & 0.67 & & & -0.24 \\
\hline 10 & -0.32 & & & -0.35 & & & & & 0.32 \\
\hline 11 & -0.33 & & & & & & & & 0.73 \\
\hline 12 & -0.36 & & & & & & & & -1.49 \\
\hline 13 & & & & & & & & & 0.95 \\
\hline 14 & & & & & & & & & 3.32 \\
\hline 15 & -0.34 & & & & & & & & 0.66 \\
\hline 16 & & & & & & & & & 1.04 \\
\hline 17 & & & & & & & & & 0.22 \\
\hline 18 & & & & & -0.47 & & & & -0.27 \\
\hline 19 & & & & & -0.32 & & & & 0.03 \\
\hline 20 & & & & & -0.74 & & & & 1.10 \\
\hline 21 & & -0.60 & & & & & & & 1.07 \\
\hline 22 & & & & & & & & & 2.04 \\
\hline 23 & -0.70 & & & & & & & & -0.35 \\
\hline 24 & -0.35 & & -0.34 & & & & & -0.35 & 0.51 \\
\hline 25 & -0.37 & -0.39 & & & & & & & 0.31 \\
\hline 26 & -0.67 & & & & & & & & -0.55 \\
\hline 27 & & -0.50 & & & & & & & 1.19 \\
\hline 28 & -0.36 & & & & & & & & -0.70 \\
\hline 29 & & & & & & & & & 1.66 \\
\hline 30 & & & & & & & & & -0.21 \\
\hline 31 & & & & & & & & & 2.42 \\
\hline 32 & & & & & & & & & 1.77 \\
\hline
\end{tabular}

Table 4.4: Optimal MIRT Exploratory Factor Analysis model for Sample 2. 
analysis. The principles "charge is conserved" and "charge does not rapidly escape to the environment" both were used only in items 1 and 2 and are labelled L3. Faraday's law and the definition of magnetic flux both loaded on items 29, 30, and 32 and are labelled L8. Corollaries $\mathrm{C} 3$ and $\mathrm{C} 4$, involving the behavior of conductors, were coded as single principles but could be derived from a number of other principles not independently tested within the instrument. As such, they were left without a derivation because their structure could not be further resolved by the instrument. There were insufficient items in the instrument to separate the addition of electric and magnetic fields (L9), so these were combined. The instrument is ambiguous about the items involving the magnetic fields of currents $(23,24$, 26, 28, and 30). While some items are represented as 3-dimensional wires $(24,30)$, some are simply shown as current into or out of the page. The expert solutions all addressed these problems using the field of an infinite straight wire and the form of the right hand rule for this system (grab wire with right hand, fingers curl in the direction of the field). Both the field of the wire and the right hand rule are derived from the more general Biot-Savart law (L6) and the right hand rule for the cross product (DF5). There were no items that made the distinction between the infinite wire field and the field of an infinitesimal current element, so the field of the wire was combined with the Biot-Savart law and the right hand rule for a wire with the right hand rule for the cross product.

Two equally likely solution paths were identified for three of the items: 7, 9, and 24 . Both solution paths were added to Table 4.5 and will be explored with MIRT. For these items, the solution path number ( 1 or 2 ) was placed in parenthesis next to the item number. For example, 7(2) in row L4 means principle L4 is used in the second solution path for item 7. If a principle was used in both solution paths, the parentheses were dropped. 
While many physics questions have multiple solution paths and one goal of physics instruction is for students to see physics as a set of linked concepts, items with multiple solution paths in an assessment instrument make it difficult to determine what the instrument actually measures. To resolve what an item with multiple common solution paths actually measures, multiple related items are required to probe the same concept in different ways. MIRT allowed the exploration of the multiple solution paths and the determination of the path measured by the instrument if the factors in each path are sufficiently probed elsewhere in the instrument.

Chapter 3 showed that placing problems in a group sharing a common stem could generate correlations between the items which were not grounded in the student's understanding of the items. Chapter 3 removed all but first item of each item group. The CSEM contains three items groups: items 3, 4, and 5; items 10 and 11; and items 17, 18, and 19. Each item group was examined to determine if spurious correlations were likely. Items 4 and 5 both depend on the answer to item 3 and cannot be treated independently. Items 4 and 5 were removed from the analysis. Item 11 depends on the answer to item 10 and was also removed. Items 17, 18 and 19 can be answered relatively independently and were retained.

Several additional items were removed from the first stage of the analysis and only analyzed after an initial optimal model was constructed: items 9, 14, 31, and 32. Item 9 was the only item directly testing Coulomb's law for the electric field (L5). The item could also be solved using Coulomb's law for the electric force (L4) and the relation of force and field $(\vec{F}=q \vec{E})$ (DF2). Many items probe these two principles. As such, first models were constructed to determine the correct structure of the electric force principles. Once this model was determined, the two solution paths for item 9 were then investigated. 
Individual experts produced multiple solution paths for items 14, 31, and 32. Some of these solutions required multiple principles not measured by other items in the CSEM. As such, items 14, 31, and 32 were not included in the initial analysis. We will call these items "reserved" items. They were analyzed after the optimal model was constructed by adding a separate "unknown" principle which captured any additional reasoning needed to solve the item. Using MIRT, the principles in Table 4.5 will be mapped onto a set of latent traits $\theta_{k i}$ representing the ability of each student $i$ to apply principle $k$.

\subsection{Model Transformation Plan}

Confirmatory analyses first fit the most complete theoretical model for a system of data and then carry out a small number of theoretically motivated transformations of the model to potentially improve model fit. Following this methodology, the most complete theoretical model (Table 4.5) of the CSEM was fit first. The expert solutions to the CSEM identified two solution paths to items 7 and 24; these alternate solutions were then explored and compared to the most complete model. The first solution path, indicated by the number in parentheses in Table 4.5, was fit as part of the initial model. The second solution path for items 7 and 24 was then fit and the best model selected for each. To test an alternate solution path, the MIRT parameter matrix is changed, constraining the parameters of the first solution path to be zero and allowing the parameters related to the alternate solution path to be non-zero. Though this cannot distinguish whether certain students are utilizing one path or the other individually, it can provide insight into which path is generally preferred by the sample of students studied. 
Table 4.5: Theoretical model of electricity and magnetism as tested by the CSEM. The "Optimal Model" indicates the samples for which the principle was included in the optimal model, M1-6, for Sample 1 and M2-6, for Sample 2. For items with two possible solution paths, the item number is followed by parentheses which enclose the solution path number. The abbreviations for the principles are law (L), definition (DF), fact $(\mathrm{F})$, corollary $(\mathrm{C})$, and lemma (LM).

\begin{tabular}{|c|c|c|c|c|}
\hline Label & $\begin{array}{l}\text { Optimal } \\
\text { Model }\end{array}$ & $\begin{array}{l}\text { Derived } \\
\text { From }\end{array}$ & CSEM\# & Principle \\
\hline \multicolumn{5}{|c|}{ Mechanics } \\
\hline DF1 & 1,2 & & $6,8,9(1)$ & The net force is the vector sum of the forces (forces add as vectors). \\
\hline L1 & 1,2 & & 10,31 & Newton's 2nd law. \\
\hline L2 & 1,2 & & $7(1), 24$ & Newton's 3rd law. \\
\hline $\mathrm{C} 1$ & & & & $\begin{array}{l}\text { Objects moving in a curved trajectory will experience } \\
\text { centripetal acceleration. }\end{array}$ \\
\hline $\mathrm{C} 2$ & 1,2 & C1, L1 & 22 & $\begin{array}{l}\text { If a particle is turning in some direction, there is a } \\
\text { force in that direction. }\end{array}$ \\
\hline \multicolumn{5}{|r|}{ Electrostatics } \\
\hline L3 & 1,2 & & 1,2 & $\begin{array}{l}\text { Charge is conserved. } \\
\text { Charge does not rapidly escape to the environment. }\end{array}$ \\
\hline F1 & 1,2 & & 2 & Charge cannot move through an insulator. \\
\hline $\mathrm{C} 3$ & 1,2 & & 13,14 & A conductor shields its interior from the electric field and force. \\
\hline $\mathrm{C} 4$ & 1,2 & & 1 & Charge spreads out over the outer surface of a conductor. \\
\hline $\mathrm{L} 4$ & 1,2 & & $3,7(2)$ & Coulomb's law for the electric force $\left(\vec{F}=\frac{k q_{1} q_{2}}{r^{2}} \hat{r}\right)$ \\
\hline L5 & 1,2 & & & Coulomb's law for the electric field $\left(\vec{E}=\frac{k q}{r^{2}} \hat{r}\right)$ \\
\hline LM1 & & L4 & $6,7,8,9(1), 14$ & Opposite charges attract/likes repel. \\
\hline DF2 & 1,2 & & $9(1), 10,12,15,19,20$ & Definition of electric field $(\vec{F}=q \vec{E})$ \\
\hline DF3 & 1,2 & & 15 & The electric field is tangent to electric field lines. \\
\hline \multicolumn{5}{|r|}{ Electric Potential } \\
\hline DF4 & 1 & & & Definition of electric potential $\left(\Delta V=\frac{W_{e x t}}{q}=-\int E d x\right)$. \\
\hline LM3 & 2 & DF4 & 16 & The electric potential contains an arbitrary constant. \\
\hline LM4 & 2 & DF4 & 17 & Relation of work and electric potential $\left(W_{e x t}=q \Delta V\right)$. \\
\hline C6 & 1,2 & DF4 & 18,20 & Relation of electric potential and field $\left(\vec{E}=-\frac{d V}{d x} \hat{x}\right)$ \\
\hline \multicolumn{5}{|r|}{ Magnetostatics } \\
\hline L6 & 1,2 & & $23,24(2), 26,28,30$ & Biot-Savart law $\left(d \vec{B}=\frac{\mu_{0}}{4 \pi} \frac{I d \vec{\ell} \times \hat{r}}{r^{2}}\right)$ \\
\hline L7 & 1,2 & & $22,24(2), 25,31$ & Lorentz force $(\vec{F}=q \vec{v} \times \vec{B}$ or $d \vec{F}=I d \vec{\ell} \times \vec{B})$ \\
\hline LM6 & & L7 & 21,27 & The magnetic force on a stationary charge is zero. \\
\hline LM7 & 1,2 & L6, L7, DF5 & $24(1)$ & Like currents attract/opposites repel. \\
\hline $\mathrm{F} 2$ & 1,2 & & 29 & The magnetic field of a permanent magnet weakens with distance. \\
\hline DF5 & 1,2 & & $22,23,24(2), 26,28,30,31$ & Right hand rule for cross products. \\
\hline DF6 & 1,2 & & 25 & $\begin{array}{l}\text { Magnitude of the cross product }(|\vec{A} \times \vec{B}|=|\vec{A}||\vec{B}| \sin \theta) \text {. } \\
\text { aduction }\end{array}$ \\
\hline L8 & 1,2 & & $29,30,32$ & $\begin{array}{l}\text { Faraday's law }\left(e m f=-\frac{d \Phi}{d t}\right) \\
\text { Definition of magnetic flux }\left(\Phi=\int_{S} \vec{B} \cdot \hat{n} d A\right) \text {. } \\
\text { erposition }\end{array}$ \\
\hline L9 & 1,2 & & $9(2), 23,28$ & Electric and magnetic fields add as vectors. \\
\hline
\end{tabular}


One of the fundamental questions about the structure of student knowledge is how granular or fine-grained the knowledge is. This can be tested by determining if the lemmas (LM) in Table 4.5 improve the model or if the model improves if the lemmas are eliminated. When a principle, such as a lemma, is removed from the model, the latent trait $\theta_{k}$ representing that principle is no longer used in the model. Removing a principle does not change the number of items in the CSEM being modelled. For the next sequence of transformations, lemmas were removed from the model by replacing them with the higher level principle from which they were derived. This was performed in three stages. First, L5 (Coulomb's force law) was combined with LM1 (opposites attract/likes repel). All items loading on either LM1 or L5 were set to load on L5. Second, lemmas involving electric potential (LM3, LM4, and LM5) were collapsed to the principle from which they were derived, DF4 (the definition of electric potential). Third, LM6 (the magnetic force on a stationary charge is zero) was combined with L7 (the Lorentz force law). Each of these was tested in turn; the order was arbitrary and could be rearranged with no effect on the results.

Finally, a model using only the bolded general categories (mechanics, electrostatics, electric potential, magnetostatics, induction, and superposition) from Table 4.5 was tested. This represented a collection of principles within general topics and was the model most closely related to previous work on evaluating the structure of the CSEM $[111,16]$. 


\subsection{Results}

\subsubsection{Constrained MIRT}

The complete model presented in Table 4.5 eliminating blocked items 4, 5, and 11 and "reserved" items 9, 14, 31, and 32, which will explored later, was fit to each sample. For items 7 and 24, where multiple likely solution paths were identified, the first solution path was used in this initial model. The model was fit by constraining the MIRT discrimination matrix, $\boldsymbol{a}_{j}$, so that discrimination parameters that did not conform to the model were zero. For example, the discrimination parameter associated with conservation of charge (L3) was constrained to be zero, $a_{L 3}=0$, except for items 1 and 2 (see Table 4.5). Following the same method as the FCI analysis, one discrimination parameter, $a_{0}$, was allowed to load on all items. This parameter is associated with a general ability, $\theta_{0}$, to solve conceptual electricity and magnetism questions. The $a_{j \neq 0}$ parameters, then, capture the additional discrimination of the item for an individual principle $j$. The initial theoretical model was fit to both samples producing models M1-1 and M2-1 where the first number is the sample number and the second number is the model number. The results of fitting this model for Sample 1 are shown in Table 4.6 and Sample 2 in Table 4.7. The models are referenced to the Transformed Model column in the tables. For both samples, the models had good fit indices: CFI $>0.96$, TLI $>0.95$, and RMSEA $<0.3$.

A sequence of more parsimonious models was then fit where transformations proceeded according to the model transformation plan in Sec. 4.8. The first transformed models, M1-2 and M2-2, investigated an alternate solution to item 7 as indicated by the $7(2)$ notation in Table 4.5 where the 2 represents the solution path number. The original model was fit with 


\begin{tabular}{|c|c|c|c|c|c|c|c|c|}
\hline $\begin{array}{c}\text { Transformed } \\
\text { Model }\end{array}$ & Transformation & $\begin{array}{c}\text { Original } \\
\text { Model }\end{array}$ & $\mathrm{AIC}$ & $\mathrm{BIC}$ & CFI & TLI & RMSEA & $\begin{array}{c}\text { Superior } \\
\text { Model }\end{array}$ \\
\hline M1-1 & & & 54,941 & 55,485 & .964 & .953 & $.025(.022, .028)$ & \\
\hline M1-2 & Solution path 2 to item 7 . & M1-1 & 54,941 & 55,485 & .964 & .953 & $.025(.022, .028)$ & M1-1 \\
\hline M1-3 & Solution path 2 to item 24 . & M1-1 & 54,928 & 55,484 & .967 & .956 & $.024(.021, .027)$ & M1-3 \\
\hline M1-4 & Combine LM1 with L4. & M1-1 & 54,914 & 55,458 & .969 & .959 & $.023(.020, .026)$ & M1-4 \\
\hline M1-5 & Combine LM3, LM4, LM5 with DF4. & M1-4 & 54,893 & 55,437 & .970 & .960 & $.023(.020, .026)$ & M1-5 \\
\hline M1-6 & Combine LM6 with L7. & M1-5 & 54,860 & 55,404 & .975 & .967 & $.021(.018, .024)$ & M1-6 \\
\hline M1-7 & Collapse to general categories. & M1-6 & 54,969 & 55,434 & .948 & .936 & $.029(.027, .032)$ & M1-6 \\
\hline
\end{tabular}

Table 4.6: Sample 1 model transformation. Differences in AIC and BIC determine whether the models are statistically different; CFI, TLI, and RMSEA indicate the quality of fit for each model.

\begin{tabular}{|c|c|c|c|c|c|c|c|c|}
\hline $\begin{array}{c}\text { Transformed } \\
\text { Model }\end{array}$ & Transformation & $\begin{array}{c}\text { Original } \\
\text { Model }\end{array}$ & $\mathrm{AIC}$ & $\mathrm{BIC}$ & CFI & TLI & RMSEA & $\begin{array}{c}\text { Superior } \\
\text { Model }\end{array}$ \\
\hline M2-1 & & & 77,330 & 77,901 & .983 & .978 & $.021(.019, .024)$ & \\
\hline M2-2 & Solution path 2 to item 7 . & M2-1 & 77,393 & 77,964 & .980 & .970 & $.023(.021, .026)$ & M2-1 \\
\hline M2-3 & Solution path 2 to item 24 . & M2-1 & 77,379 & 77,962 & .978 & .970 & $.025(.022, .027)$ & M2-1 \\
\hline $\mathrm{M} 2-4$ & Combine LM1 with L4. & M2-1 & 77,282 & 77,853 & .983 & .978 & $.021(.019, .024)$ & M2-4 \\
\hline M2-5 & Combine LM3, LM4, LM5 with DF4. & M2-4 & 77,308 & 77,879 & .984 & .980 & $.021(.018, .023)$ & M2-4 \\
\hline M2-6 & Combine LM6 with L7. & $\mathrm{M} 2-4$ & 77,265 & 77,835 & .984 & .980 & $.020(.018, .023)$ & M2-6 \\
\hline M2-7 & Collapse to general categories. & M2-6 & 77,315 & 77,803 & .975 & .969 & $.025(.023, .028)$ & M2-6 \\
\hline
\end{tabular}

Table 4.7: Sample 2 model transformation. Differences in AIC and BIC determine whether the models are statistically different; CFI, TLI, and RMSEA indicate the quality of fit for each model.

the $7(1)$ constraints from Table 4.5. Item 7 asks for the magnitude and direction of the forces on unequal point charges. Solution path 1 used opposites attract/likes repel (LM1) and Newton's 3rd law (L2). The second solution path also used opposites attract/likes repel but applied Coulomb's force law (L4) to obtain the magnitude. In Sample 1, there was no difference in the fit of the two solution paths. In Sample 2, the model fit was significantly worse for the second solution with an increase in both AIC and BIC of 63 , a very strong change using Raftery's classification [98]. As such, the model with the first solution path was retained in both cases implying that students' solve item 7 using opposites attract/likes repel (LM1) and Newton's 3rd law (L2) rather than applying Coulomb's law to obtain the magnitude. Tables 4.6 and 4.7 show the fit parameters for the transformed model, the model from which it was transformed and is being compared (Original Model), and which of the models was retained (Superior Model). 
Models M1-3 and M2-3 investigated an alternate solution path to item 24. The first solution path, used in the initial model, solved the item by applying like currents attract/opposites repel (LM7) and Newton's 3rd law (L2). The second solution path began with first principles from the Biot-Savart law (L6) and applied the Lorentz force law (L7) using the right-hand-rule for the cross product (DF5) to find the direction. Newton's 3rd law (L2) was again applied to find the second force. Solution path 2 showed a significant improvement in AIC of 13 for Sample 1, a very strong change, but no significant change in BIC. For Sample 2, the second solution path was significantly worse with AIC increasing by 49 and BIC by 61, both very strong changes. With only the change in AIC in Sample 1 supporting solution path 2 and much stronger changes in Sample 2 supporting path 1, path 1 was retained for all future models. As such, students solve item 24 by applying like currents attract/opposites repel (LM7) rather than the more fundamental Biot-Savart law.

Models M1-4/M2-4 through M1-6/M2-6 test whether condensing some of the lemmas into broader principles, laws and definitions, improves model fit. Model M1-4/M2-4 replaces opposites attract/likes repel (LM1) with Coulomb's force law (L4) from which it is derived. This significantly improved model fit over M1-1 in Sample 1 with AIC and BIC decreasing by 27 , both very strong changes. In Sample 2, model fit was also improved when compared to the model M2-1 with AIC decreasing by 48 and BIC by 48, both very strong changes. As such, transformed models M1-4/M2-4 were retained as the superior models. This change also served to collapse the two solution paths for item 7 into one path. As such, students understanding of the electric force was less granular than initially represented in the theoretical model.

The next models, M1-5/M2-5, combined several principles of electric potential (LM3, 
LM4, LM5) into the definition of electric potential (DF4) from which they were derived while retaining the changes made in model M1-4/M2-4. This model was a significant improvement over model M1-4 in Sample 1 with AIC and BIC decreasing by 21, both very strong changes. However, in Sample 2, model M2-5 was significantly inferior to model M2-4 with AIC and BIC increasing by 26, both very strong changes. As such, model M1-5 was retaining for Sample 1 as the superior model, but not for Sample 2. This marked the first substantial deviation between the two data sets. For Sample 1, students had a more integrated understanding of potential allowing the combination of LM3 (potential contains an arbitrary constant), LM4 (the relation of work and potential), and LM5 (electric field points to lower potential) into a single definition of potential (DF4). Students in Sample 2 had differing reasoning abilities on these lemmas.

Models M1-6/M2-6 combined the principle that the magnetic force on a stationary charge is zero (LM6) with the principle from which it is derived, the Lorentz force law for magnetic fields (L7). This change significantly improved model fit for model M1-5 in Sample 1 with AIC decreasing by 33 and BIC by 33, both very strong changes. In Sample 2, model M2-6, which made the same modifications to model M2-4, significantly improved model fit with AIC decreasing by 17 and BIC by 18, both very strong changes. As such, models M1-6/M2-6 were retained as the superior models. The reasoning of students in both samples about stationary magnetic force was not differentiated from reasoning about non-zero magnetic force.

Models M1-6 and M2-6 represent the most parsimonious models which the authors felt could be theoretically justified. Many studies have sought to produce even more general models of the FCI and the FMCE through exploratory methods such as factor analysis as 
described in the introduction. These methods model the internal structure of an instrument through a small number of factors thought to represent subsets of the instrument which are conceptually similar. To test whether this was the correct way to model the CSEM, models M1-7/M2-7 condensed models M1-6/M2-6 to the bolded general categories in Table 4.5 (mechanics, electrostatics, electric potential, magnetostatics, induction, and superposition). Model M1-7 had significantly poorer fit than model M1-6 with an increase in AIC of 109 and BIC of 30, both very strong changes. Therefore, Model 1-6 represents the optimal model of student knowledge for Sample 1. Model M2-7 made a similar transformation to model M2-6; the model fit indices to this transformation were mixed. AIC increased by 50, but BIC decreased by 32, both very strong changes; however, CFI, TLI, and RMSEA all support the choice of Model M2-6 as the optimal model for Sample 2. For both samples, the theoretical model grounded in specific principles of physics was superior to a model using broad general topics.

The sequence of models used progressively fewer parameters; model fit usually increases with the addition of parameters. AIC and BIC both penalize the addition of parameters to correct for overfitting. BIC penalizes additional parameters more strongly than AIC.

\subsubsection{Analysis of Optimal Models}

The full optimal models for Sample 1, model M1-6, and Sample 2, M2-6, are shown in Table 4.8. Each item is reported with the individual principles required for its solution. The number in parenthesis is the discrimination, $a_{j k}$, of item $j$ on principle $k$. The mean and standard error of the mean of the discriminations are reported and were calculated by bootstrapping with 100 sub-samples. The principle discrimination, $a_{j k}$, represents how well 
Table 4.8: Optimal MIRT model for Samples 1 and 2. The first column shows the CSEM item number (\#). Not all CSEM items were modelled. The number in parenthesis is the discrimination, $a_{j k}$, for principle $k$ of item $j . a_{j 0}$ is the discrimination for a factor loaded on all items and $d_{j}$ is related to the overall difficulty of the item. Both parameters are also rank ordered from smallest to largest. The significance of a $t$-test with Bonferroni correction to determine if the difficulty and discrimination are different from zero is reported as a superscript. A superscript of "a" represents the corrected equivalent of $p<0.05$, "b" $p<0.01$, and "c" $p<0.001$.

\begin{tabular}{|c|c|c|c|c|c|c|c|c|c|c|}
\hline \multirow[b]{2}{*}{ \# } & \multicolumn{5}{|c|}{ Sample 1} & \multicolumn{5}{|c|}{ Sample 2} \\
\hline & Principles & $a_{j 0}$ & $d_{j}$ & $\begin{array}{c}a_{j 0} \\
\text { Rank }\end{array}$ & $\begin{array}{c}d_{j} \\
\text { Rank }\end{array}$ & Principles & $a_{j 0}$ & $d_{j}$ & $\begin{array}{c}a_{j 0} \\
\text { Rank }\end{array}$ & $\begin{array}{c}d_{j} \\
\text { Rank }\end{array}$ \\
\hline 1 & $\mathrm{C} 4(0.16)^{c} \mathrm{~L} 3(0.48)^{c}$ & $0.68^{c}$ & $2.67^{c}$ & 8 & 12 & $\mathrm{C} 4(0.22)^{c} \mathrm{~L} 3(0.47)^{c}$ & $0.82^{c}$ & $1.38^{c}$ & 11 & 23 \\
\hline 2 & $\mathrm{~F} 1(0.23)^{c} \mathrm{~L} 3(0.46)^{c}$ & $0.58^{c}$ & $0.98^{c}$ & 5 & 10 & $\mathrm{~F} 1(0.27)^{c} \mathrm{~L} 3(0.49)^{c}$ & $0.59^{c}$ & $-0.66^{c}$ & 6 & 9 \\
\hline 3 & $\mathrm{~L} 4(0.67)^{c}$ & $0.81^{c}$ & $2.04^{c}$ & 12 & 19 & $\mathrm{~L} 4(0.32)^{c}$ & $0.66^{c}$ & $1.58^{c}$ & 7 & 24 \\
\hline 6 & $\mathrm{~L} 4(0.41)^{c} \mathrm{DF} 1(0.08)^{c}$ & $1.23^{c}$ & $2.05^{c}$ & 22 & 20 & $\mathrm{~L} 4(0.33)^{c} \mathrm{DF} 1(0.18)^{c}$ & $1.39^{c}$ & $0.99^{c}$ & 21 & 21 \\
\hline 7 & $\mathrm{~L} 4(-0.15)^{c} \mathrm{~L} 2(0.15)^{c}$ & $1.25^{c}$ & $1.23^{c}$ & 23 & 15 & $\mathrm{~L} 4(-0.01) \mathrm{L} 2(0.59)^{c}$ & $2.03^{c}$ & $-0.05^{c}$ & 25 & 15 \\
\hline 8 & $\mathrm{~L} 4(0.26)^{c} \mathrm{DF} 1(0.13)^{c}$ & $0.86^{c}$ & $1.15^{c}$ & 15 & 12 & $\mathrm{~L} 4(0.22)^{c} \mathrm{DF} 1(0.20)^{c}$ & $0.97^{c}$ & $0.62^{c}$ & 15 & 19 \\
\hline 10 & $\mathrm{~L} 1(0.31)^{c} \mathrm{DF} 2(0.23)^{c}$ & $1.09^{c}$ & $0.23^{c}$ & 19 & 5 & $\mathrm{~L} 1(0.25)^{c} \mathrm{DF} 2(0.10)^{c}$ & $1.30^{c}$ & $-0.50^{c}$ & 19 & 11 \\
\hline 12 & DF2 $(0.28)^{c}$ & $0.78^{c}$ & $2.66^{c}$ & 11 & 21 & $\mathrm{DF} 2(0.15)^{c}$ & $0.94^{c}$ & $1.68^{c}$ & 13 & 25 \\
\hline 13 & $\mathrm{C} 3(0.21)^{c}$ & $0.94^{c}$ & $1.57^{c}$ & 18 & 17 & $\mathrm{C} 3(0.25)^{c}$ & $0.81^{c}$ & $-0.97^{c}$ & 10 & 7 \\
\hline 15 & DF2 $(0.07)^{c}$ DF3 $(0.26)^{c}$ & $0.93^{c}$ & $0.72^{c}$ & 16 & 9 & DF2 $(0.06)^{c}$ DF3 $(0.22)^{c}$ & $1.26^{c}$ & $-0.94^{c}$ & 18 & 8 \\
\hline 16 & $\mathrm{DF} 4(0.31)^{c}$ & $0.82^{c}$ & $0.54^{c}$ & 13 & 7 & $\operatorname{LM} 3(0.27)^{c}$ & $0.95^{c}$ & $-1.16^{c}$ & 14 & 5 \\
\hline 17 & $\mathrm{DF} 4(0.19)^{c}$ & $0.69^{c}$ & $1.36^{c}$ & 9 & 16 & $\mathrm{LM} 4(0.27)^{c}$ & $1.15^{c}$ & $-0.31^{c}$ & 16 & 13 \\
\hline 18 & $\mathrm{C} 6(1.06)^{c}$ & $0.40^{c}$ & $1.18^{c}$ & 3 & 14 & $\mathrm{C} 6(0.82)^{c}$ & $0.30^{c}$ & $0.32^{c}$ & 2 & 17 \\
\hline 19 & $\mathrm{DF} 4(0.91)^{c} \mathrm{DF} 2(0.13)^{c}$ & $1.13^{c}$ & $2.80^{c}$ & 21 & 24 & DF2 $(0.12)^{c} \operatorname{LM} 5(0.50)^{c}$ & $1.40^{c}$ & $-0.05^{c}$ & 22 & 14 \\
\hline 20 & $\mathrm{DF} 4(0.45)^{c}$ DF2(0.02) C6 $(1.00)^{c}$ & $0.49^{c}$ & $-0.62^{c}$ & 4 & 1 & $\mathrm{DF} 2(0.17)^{c} \mathrm{C} 6(0.75)^{c} \operatorname{LM} 5(0.39)^{c}$ & $0.55^{c}$ & $-2.10^{c}$ & 4 & 1 \\
\hline 21 & $\mathrm{~L} 7(1.03)^{c}$ & $0.75^{c}$ & $1.68^{c}$ & 10 & 18 & $\mathrm{~L} 7(0.61)^{c}$ & $0.40^{c}$ & $-1.40^{c}$ & 3 & 4 \\
\hline 22 & $\mathrm{C} 2(0.20)^{c} \mathrm{~L} 7(0.19)^{c} \mathrm{DF} 5(0.23)^{c}$ & $0.38^{c}$ & $-0.49^{c}$ & 1 & 2 & $\mathrm{C} 2(0.23)^{c} \operatorname{L} 7(0.04)^{c}$ DF5 $(0.08)^{c}$ & $0.02^{c}$ & $-0.53^{c}$ & 1 & 10 \\
\hline 23 & L9(0.01) DF5 $(0.26)^{c} \mathrm{~L} 6(0.44)^{c}$ & $1.12^{c}$ & $2.79^{c}$ & 20 & 23 & $\mathrm{~L} 9(0.21)^{c} \operatorname{DF} 5(0.25)^{c} \operatorname{L} 6(0.45)^{c}$ & $1.42^{c}$ & $0.74^{c}$ & 23 & 20 \\
\hline 24 & $\mathrm{~L} 2(0.13)^{c} \operatorname{LM} 7(0.27)^{c}$ & $0.85^{c}$ & $-0.15^{c}$ & 14 & 3 & $\mathrm{~L} 2(0.58)^{c} \operatorname{LM} 7(0.31)^{c}$ & $1.35^{c}$ & $-0.97^{c}$ & 20 & 6 \\
\hline 25 & $\operatorname{L} 7(0.30)^{c} \operatorname{DF} 6(0.33)^{c}$ & $0.93^{c}$ & $0.49^{c}$ & 17 & 6 & $\mathrm{~L} 7(0.37)^{c} \mathrm{DF} 6(0.32)^{c}$ & $1.16^{c}$ & $-0.48^{c}$ & 17 & 12 \\
\hline 26 & DF5 $(0.39)^{c} \operatorname{L} 6(0.52)^{c}$ & $1.64^{c}$ & $3.71^{c}$ & 25 & 25 & $\operatorname{DF} 5(0.25)^{c} \operatorname{L} 6(0.45)^{c}$ & $1.69^{c}$ & $1.22^{c}$ & 24 & 22 \\
\hline 27 & $\mathrm{~L} 7(0.68)^{c}$ & $0.67^{c}$ & $1.18^{c}$ & 7 & 13 & $\mathrm{~L} 7(0.54)^{c}$ & $0.72^{c}$ & $-1.55^{c}$ & 9 & 3 \\
\hline 28 & $\mathrm{~L} 9(0.24)^{c} \mathrm{DF} 5(0.18)^{c} \mathrm{~L} 6(0.09)^{c}$ & $0.40^{c}$ & $0.64^{c}$ & 2 & 8 & $\mathrm{~L} 9(0.21)^{c} \operatorname{DF} 5(0.07)^{c} \operatorname{L} 6(0.08)^{c}$ & $0.56^{c}$ & $0.57^{c}$ & 5 & 18 \\
\hline 29 & $\mathrm{~L} 8(0.33)^{c} \mathrm{~F} 2(0.23)^{c}$ & $1.27^{c}$ & $-0.10^{c}$ & 24 & 4 & $\mathrm{~L} 8(0.23)^{c} \mathrm{~F} 2(0.17)^{c}$ & $0.84^{c}$ & $-1.83^{c}$ & 12 & 2 \\
\hline 30 & DF5 $(0.08)^{c} \operatorname{L} 6(0.08)^{c} \operatorname{L} 8(0.24)^{c}$ & $0.66^{c}$ & $1.02^{c}$ & 6 & 11 & DF5 $(0.06)^{c} \operatorname{L} 6(0.03)^{c} \operatorname{L} 8(0.31)^{c}$ & $0.68^{c}$ & $0.16^{c}$ & 8 & 16 \\
\hline
\end{tabular}

the item, $j$, discriminates between high and low ability students above the discrimination, $a_{j 0}$, of the item on a general facility with conceptual electricity and magnetism. Table 4.8 also reports the results of a $t$-test for each discrimination as a superscript to determine if the discrimination parameter is significantly different from zero. A Bonferroni correction has been applied to adjust for the number of statistical tests performed. The table also reports $d_{j}$, the overall difficulty of the item.

The optimal models for Sample 1 and Sample 2 differ slightly because of the way electric potential was modelled. For Sample 1, only DF4 was included (model M1-5), but 
in Sample 2 DF4 was expanded into lemmas LM3, LM4, and LM5 (model M2-4). These differences were retained as optimal models M1-6 and M2-6 were constructed. To determine how similar the models are, a single model must be selected. Because model M1-6 is the more parsimonious, it was selected for comparisons between the two samples. This model was fit to Sample 2 and bootstrapping was repeated. A comparison of the fits of this model for the two samples is shown in Table 4.9 where the mean fit values for Sample 2 have been subtracted from those obtained from the fit of Sample 1 to form $\Delta a_{j k}$ and $\Delta d_{j}$. The significance of the differences between the parameters was calculated with $t$-tests with a Bonferroni correction. Significance values are reported as superscripts. The difference in overall discrimination, $\Delta a_{j 0}$, and difficulty, $\Delta d_{j}$, is statistically significant $(p \mathbf{s}<0.001)$ for the majority of the items. Many of the principle discriminations, $a_{j k}$, were not significantly different between the samples, suggesting that many of CSEM items perform similarly at different institutions once overall differences in ability are removed.

For Sample 1, items 3,12, 13, 16, 17, 18, 21, and 27 load on a single principle and probe six individual concepts: L4, DF2, C3, DF4, C6, and L7. These questions could be used to investigate student knowledge about these concepts independent of other principles. The remaining 19 items load on multiple principles; however, many have a single principle that has a discrimination at least twice as large in absolute value as the next largest (items 1, $2,6,8,15,19,20,24$, and 30$)$. These items could be used to characterize student knowledge on the high discrimination principle.

For Sample 2, items 3, 12, 13, 16, 17, 18, 21, and 27 also load on a single factor. The remaining 19 items load on multiple principles; however, many have a single factor that has a discrimination at least twice as large in absolute value as the next largest (items 1, 7, 10, 
Table 4.9: The difference in parameters between samples 1 and 2 using the the optimal model for Sample 1 (model M1-6). The number in parenthesis is the difference in discrimination, $\Delta a_{j k}$, for item $j . \Delta a_{j 0}$ is the difference in discrimination for a principle loaded on all items and $\Delta d_{j}$ is the difference in the difficulty of the item. Each difference was t-tested with a Bonferroni correction. A superscript of "a" represents the corrected equivalent of $p<0.05$, "b" $p<0.01$, and "c" $p<0.001$.

\begin{tabular}{lccc}
\hline CSEM\# & Principles & $\Delta a_{j 0}$ & $\Delta d_{j}$ \\
\hline 1 & $\mathrm{C} 4(-0.06 \pm 0.01)^{a} \mathrm{~L} 3(0.02 \pm 0.02)$ & $-0.13 \pm 0.01^{c}$ & $1.28 \pm 0.02^{c}$ \\
2 & $\mathrm{~F} 1(-0.10 \pm 0.02)^{b} \mathrm{~L} 3(-0.02 \pm 0.02)$ & $-0.02 \pm 0.01$ & $1.65 \pm 0.01^{c}$ \\
3 & $\mathrm{~L} 4(0.38 \pm 0.02)^{c}$ & $0.18 \pm 0.01^{c}$ & $0.50 \pm 0.02^{c}$ \\
6 & $\mathrm{~L} 4(0.07 \pm 0.01)^{a} \mathrm{DF} 1(-0.11 \pm 0.01)^{c}$ & $-0.14 \pm 0.01^{c}$ & $1.07 \pm 0.02^{c}$ \\
7 & $\mathrm{~L} 4(-0.16 \pm 0.01)^{c} \mathrm{~L} 2(-0.45 \pm 0.01)^{c}$ & $-0.77 \pm 0.02^{c}$ & $1.29 \pm 0.01^{c}$ \\
8 & $\mathrm{~L} 4(0.05 \pm 0.01)^{a} \mathrm{DF} 1(-0.06 \pm 0.01)^{a}$ & $-0.10 \pm 0.01^{c}$ & $0.56 \pm 0.01^{c}$ \\
10 & $\mathrm{~L} 1(0.04 \pm 0.02) \mathrm{DF} 2(0.13 \pm 0.01)^{c}$ & $-0.21 \pm 0.01^{c}$ & $0.73 \pm 0.01^{c}$ \\
12 & $\mathrm{DF} 2(0.13 \pm 0.01)^{c}$ & $-0.15 \pm 0.01^{c}$ & $0.99 \pm 0.02^{c}$ \\
13 & $\mathrm{C} 3(0.01 \pm 0.01)^{c}$ & $0.15 \pm 0.01^{c}$ & $2.53 \pm 0.01^{c}$ \\
15 & $\mathrm{DF} 2(0.02 \pm 0.01) \mathrm{DF} 3(0.05 \pm 0.02)$ & $-0.34 \pm 0.01^{c}$ & $1.67 \pm 0.01^{c}$ \\
16 & $\mathrm{DF} 4(0.18 \pm 0.01)^{c}$ & $-0.01 \pm 0.01$ & $1.59 \pm 0.01^{c}$ \\
17 & $\mathrm{DF} 4(0.05 \pm 0.01)^{c}$ & $-0.32 \pm 0.01^{c}$ & $1.65 \pm 0.01^{c}$ \\
18 & $\mathrm{C} 6(0.22 \pm 0.02)^{c}$ & $0.10 \pm 0.01^{c}$ & $0.87 \pm 0.01^{c}$ \\
19 & $\mathrm{DF} 4(0.60 \pm 0.02)^{c} \mathrm{DF} 2(-0.05 \pm 0.01)$ & $-0.12 \pm 0.02^{c}$ & $2.87 \pm 0.03^{c}$ \\
20 & $\mathrm{LF} 4(0.12 \pm 0.01)^{c} \mathrm{DF} 2(-0.22 \pm 0.01)^{c} \mathrm{C} 6(0.25 \pm 0.02)^{c}$ & $-0.02 \pm 0.01$ & $1.50 \pm 0.02^{c}$ \\
21 & $\mathrm{~L} 7(0.39 \pm 0.02)^{c}$ & $0.37 \pm 0.01^{c}$ & $3.10 \pm 0.02^{c}$ \\
22 & $\mathrm{C} 2\left(-0.01 \pm 0.01 \mathrm{~L}^{c}(0.16 \pm 0.01)^{c} \mathrm{DF} 5(0.15 \pm 0.01)^{c}\right.$ & $0.36 \pm 0.01^{c}$ & $0.03 \pm 0.01^{b}$ \\
23 & $\mathrm{~L} 9(-0.20 \pm 0.01)^{c} \mathrm{DF} 5(0.00 \pm 0.02) \mathrm{L} 6(0.03 \pm 0.02)$ & $-0.28 \pm 0.01^{c}$ & $2.07 \pm 0.02^{c}$ \\
24 & $\mathrm{~L} 2(-0.46 \pm 0.01)^{c} \mathrm{LM} 7(-0.03 \pm 0.02)$ & $-0.48 \pm 0.01^{c}$ & $0.83 \pm 0.01^{c}$ \\
25 & $\mathrm{~L} 7(-0.07 \pm 0.01)^{c} \mathrm{DF} 6(0.06 \pm 0.02)$ & $-0.23 \pm 0.01^{c}$ & $0.97 \pm 0.01^{c}$ \\
26 & $\mathrm{DF} 5(0.11 \pm 0.02)^{b} \mathrm{~L} 6(0.11 \pm 0.02)$ & $-0.05 \pm 0.02$ & $2.52 \pm 0.04^{c}$ \\
27 & $\mathrm{~L} 7(0.13 \pm 0.01)^{c}$ & $-0.07 \pm 0.01^{c}$ & $2.73 \pm 0.01^{c}$ \\
28 & $\mathrm{~L}(0.011 \pm 0.01)^{c} \mathrm{~L} 6(0.02 \pm 0.01)$ & $-0.15 \pm 0.01^{c}$ & $0.09 \pm 0.01^{c}$ \\
29 & $\mathrm{~L} 8(0.08 \pm 0.01)^{c} \mathrm{~F} 2(0.04 \pm 0.01)$ & $0.41 \pm 0.01^{c}$ & $1.78 \pm 0.01^{c}$ \\
30 & $\mathrm{DF} 9(0.00 \pm 0.01) \mathrm{L} 6(0.07 \pm 0.01)^{c} \mathrm{~L} 8(-0.07 \pm 0.01)^{b}$ & $-0.03 \pm 0.01$ & $0.87 \pm 0.01^{c}$ \\
\hline
\end{tabular}

$15,19,22,28$, and 30).

\subsubsection{Correlation of Abilities}

The "mirt" package was used to estimate the ability, $\theta_{i k}$, of each student $i$ to apply principle $k$. The correlation of these abilities for Sample 1 is shown in Fig. 4.1 and for Sample 2 in Fig. 4.2. For both samples, while some groups of strongly correlated abilities were present, in general most abilities were weakly or negatively correlated suggesting the 
students have not developed an integrated understanding of the material.

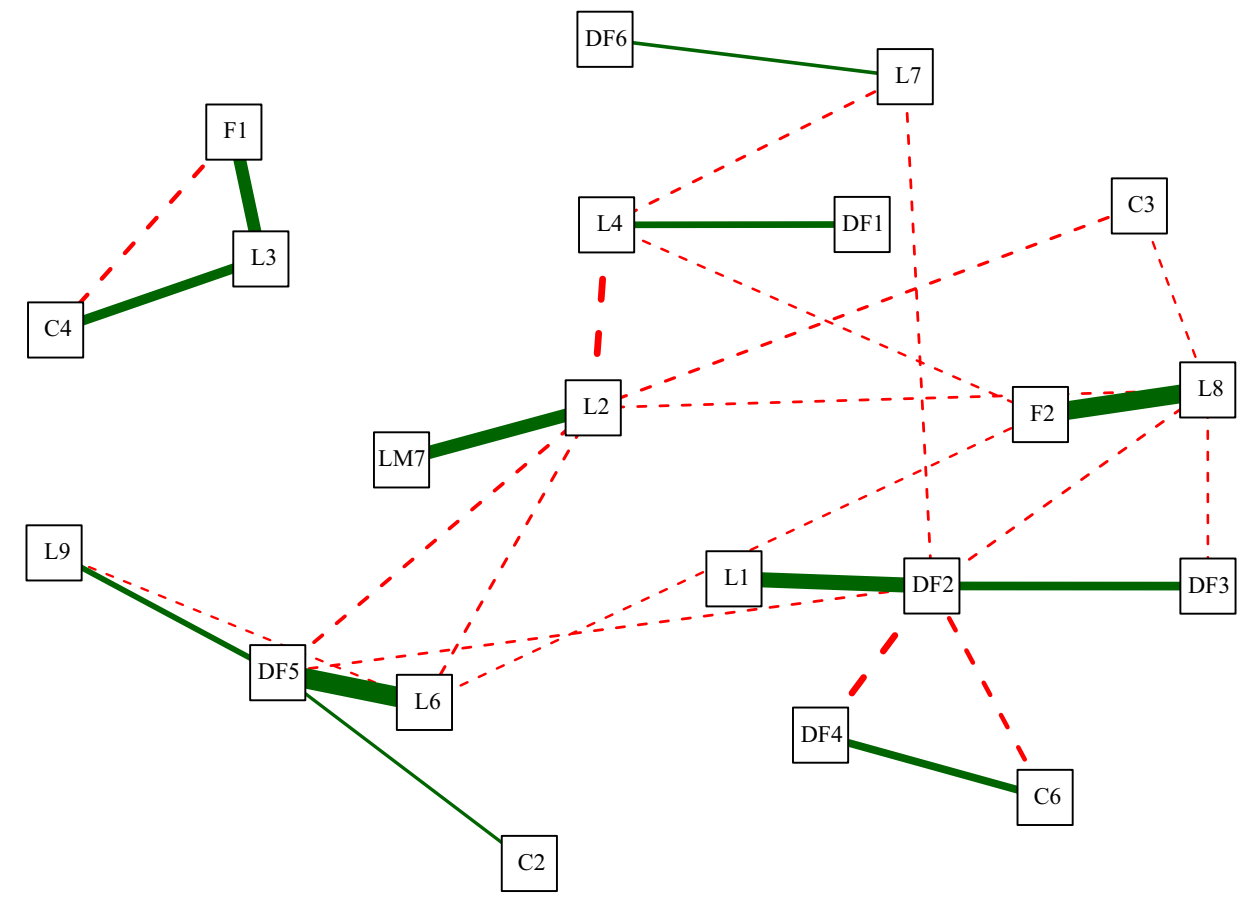

Figure 4.1: Correlation matrix for student ability on each principle in the optimal model for Sample 1. Line thickness represents the size of the correlation. Solid (green) lines represent positive correlations; dashed (red) lines negative correlations.

\subsubsection{Analysis of Reserved Items}

Item 9 was initially withheld from the analysis because the expert solutions provided two equally plausible solution paths, one relying primarily on reasoning using electric force, the other relying on reasoning using the electric field. Only item 9 directly probed the electric field of a point charge (L5). As such, an optimal model for other principles was identified before exploring item 9 . Two models were tested using the two possible solutions to item 9 as shown in Table 4.5. The first solution path used a positive test charge, opposites attacts/likes repel (LM1, now L4), the vector addition of forces (DF1), and the relation of force and field 
Table 4.10: Sample 1 and 2 reserved item comparisons. Differences in AIC and BIC determine whether the models are statistically different; CFI, TLI, and RMSEA indicate the quality of fit for each model.

\begin{tabular}{|c|c|c|c|c|c|c|c|}
\hline CSEM\# & $\begin{array}{c}\text { Solution } \\
\text { Path }\end{array}$ & AIC & BIC & CFI & TLI & RMSEA & $\begin{array}{c}\text { Superior } \\
\text { Path }\end{array}$ \\
\hline \multicolumn{8}{|c|}{ Sample 1} \\
\hline \multirow{2}{*}{9} & Path 1 & 56,675 & 57,247 & .973 & .965 & $.022(.020, .025)$ & \multirow{2}{*}{ Path 1} \\
\hline & Path 2 & 56,691 & 57,258 & .973 & .964 & $.022(.019, .025)$ & \\
\hline \multirow[b]{2}{*}{14} & Path 1 & 57,184 & 57,750 & .970 & .961 & $.022(.019, .025)$ & \multirow{2}{*}{ Path 1} \\
\hline & Path 2 & 57,188 & 57,760 & .971 & .962 & $.022(.019, .025)$ & \\
\hline \multirow{2}{*}{31} & Path 1 & 56,925 & 57,492 & .967 & .957 & $.024(.021, .027)$ & \multirow{2}{*}{ Path 2} \\
\hline & Path 2 & 56,910 & 57,482 & .967 & .957 & $.024(.021, .027)$ & \\
\hline \multirow{2}{*}{32} & Path 1 & 57,661 & 58,221 & .970 & .961 & $.022(.019, .025)$ & \multirow{2}{*}{ Path 1} \\
\hline & Path 2 & 57,680 & 59,246 & .970 & .961 & $.022(.020, .025)$ & \\
\hline \multicolumn{8}{|c|}{ Sample 2} \\
\hline \multirow{2}{*}{9} & Path 1 & 80,398 & 80,998 & .986 & .982 & $.019(.017, .022)$ & \multirow{2}{*}{ Path 2} \\
\hline & Path 2 & 80,393 & 80,988 & .986 & .982 & $.019(.017, .022)$ & \\
\hline \multirow{2}{*}{14} & Path 1 & 79,457 & 80,051 & .987 & .983 & $.018(.015, .020)$ & \multirow{2}{*}{ Path 1} \\
\hline & Path 2 & 79,497 & 80,097 & .986 & .981 & $.019(.016, .021)$ & \\
\hline \multirow{2}{*}{31} & Path 1 & 79,073 & 79,667 & 987 & .983 & $.018(.016, .021)$ & \multirow{2}{*}{ Path 2} \\
\hline & Path 2 & 79,044 & 79,644 & .985 & .981 & $.019(.017, .022)$ & \\
\hline & Path 1 & 80,703 & 81,291 & .985 & .981 & $.019(.017, .022)$ & \multirow{2}{*}{ Path 1} \\
\hline & Path 2 & 80,725 & 81,319 & .984 & .979 & $.020(.017, .022)$ & \\
\hline
\end{tabular}




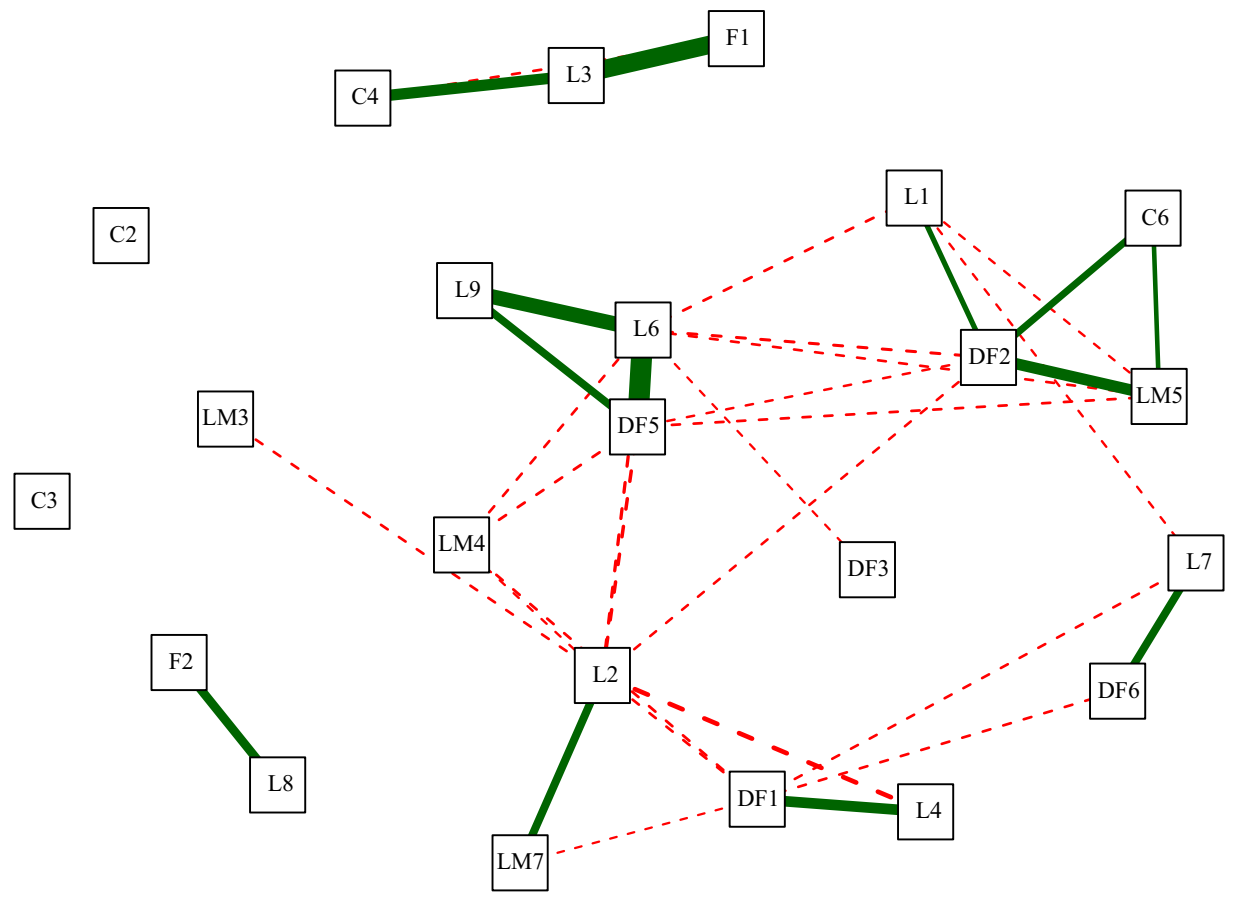

Figure 4.2: Correlation matrix for student ability on each principle in the optimal model for Sample 2. Line thickness represents the size of the correlation. Solid (green) lines represent positive correlations; dashed (red) lines negative correlations.

(DF2). The second solution used that electric field points away from positive charge (LM2) and the vector addition of fields (L9). Solution path 1 involving electric force produced the optimal model for Sample 1 with very strong changes in AIC and BIC. Solution path 2 involving electric field produced the superior model for Sample 2 with a significant change in AIC and a strong change in BIC. Model fit statistics and the solution path selected for analysis in this section are shown in Table 4.10. Note, AIC and BIC for this section cannot be compared directly to values in Table 4.6 and 4.7 because the number of items fit have changed.

Items 14,31 , and 32 were investigated by adding a separate unknown principle to their model in Table 4.5. Expert solutions were quite varied for these items and often contained 
additional principles not tested elsewhere in the instrument. Models with this additional principle were tested independently and compared for each item. The unknown principle was used to capture any reasoning not already captured by the principles included in Table 4.5. First, the loadings for items 14, 31, and 32 presented in Table 4.5 were added to the already identified optimal models M1-6/M2-6 and model fit recalculated. These models are identified as solution path 1 . The fit of this model was then compared to a model that added the unknown principle to one of the reserved items, solution path 2. In both Sample 1 and Sample 2, the addition of the unknown principle only improved the model fit for item 31 implying the students were using additional reasoning beyond L7 and DF5 to solve the item.

\subsection{Discussion}

This chapter investigated two research questions:

RQ1: What is the optimal model of student knowledge measured by the CSEM? Are the principles forming the optimal model consistent across samples? The optimal model for Sample 1 required 23 principles, while the optimal model for Sample 2 required 25. The optimal model had 22 principles in common. As such, while there were some differences between the two optimal models, in general they were very similar despite large differences in instructional environment and the student's overall performance on the CSEM.

For Sample 1 the optimal model collapsed most of the lemmas into the higher level principles from which they were derived. Two lemmas, LM2 and LM7, were retained in optimal model M1-6. The collapse of LM2 into L5 could not be investigated because the CSEM did not contain other items which employed L5. For this sample, student knowledge 
of electricity and magnetism is better represented by the general laws, definitions, facts, and corollaries defining the topic without the additional set of qualitative principles. This observation is consistent with the results found in Chapter 3 for the FCI.

The optimal model for Sample 2 included three additional lemmas (LM3, LM4, and LM5) rather than the general definition of electric potential (DF4). In all, 5 of the original 7 lemmas were retained in optimal model M2-6. Students in Sample 2 have a less integrated understanding of electric potential than students in Sample 1, perhaps explaining their overall weaker performance on the CSEM. For these students, a model with detailed coverage of the implications of the general laws better fit the student understanding of electricity and magnetism. The understanding of these students is less well integrated than that of students in Sample 1.

Collapsing the optimal models further to very general categories such as electrostatics or magnetostatics (models M1-7/M2-7) reduced model fit, implying student knowledge of electricity and magnetism is more granular than these broad topics.

The models of the two samples also differed for reserved item 9; this may have resulted from the instruction provided to students in the two samples. The lead instructor for Sample 1 reported presenting the material from the standpoint of inserting a positive test charge; the solution path using electric force produced the optimal model for item 9 in Sample 1. Conversely, many instructors taught the classes in Sample 2 and presented the addition of electric field in many different ways. In this case, the solution using the principle that fields point away from positive charges produced the superior model. This suggests that MIRT could be used to probe differences in the effect of specific instructional choices on student understanding. 
Exploration of the rest of the reserved items (14, 31, and 32) through the addition of an unknown principle showed that these items could reasonably be explained using the theoretical model already developed for this instrument. With the exception of item 31, none of the models including the unknown factor performed better than the ones without it. Again, the optimal models for the two samples were similar but not identical.

The differences between the optimal models for the two samples shows the optimal model for the CSEM does vary somewhat between institutions. The difference, however, was restricted to the decision to retain lemmas LM3, LM4, and LM5. Further, unlike the FCI, both optimal models of the CSEM did include one lemma, LM7, and potentially a second lemma, LM2; combining LM2 with L5 could not be tested because of the structure of the instrument. While model M2-5 and models M1-3/M2-3 were significantly less well fitting, they still possessed excellent fit characteristics with CFI $>0.96$, TLI $>0.95$, and RMSEA $<0.25$. As such, while variations between institutions were present, in each case the optimal models produced excellent fit statistics. It may, therefore, be reasonable to use the model of the CSEM eliminating all lemmas when comparing results between institutions.

RQ2: Are the parameters of the optimal models consistent between samples? The uniformly larger difficulty values, $d_{j}$, in Sample 1 indicate that the CSEM was a much easier test for students in this sample. All differences in overall difficulty, $\Delta d_{j}$, were statistically significant in Table 4.9. This difference was expected as the students in Sample 1 were generally higher performing with higher overall CSEM post-test scores than students in Sample 2. The instructional environment in Sample 1 was also more controlled and enriched and should have lead to stronger learning outcomes. While most of the overall discriminations, $a_{j 0}$, of the items were significantly different between samples, only items $7,10,15,17,21,22$, 
23, 24, 25, and 29 had differences in overall discrimination greater than 0.2 , approximately one third of the items; therefore, most of the discrimination differences were fairly small.

In general, most items had overall discriminations $a_{j 0}$ and principle discriminations $a_{j k}$ that suggested the items were both well functioning with positive discrimination values. Only item 7 in Sample 1 had a principle discrimination less than zero suggesting that it may not be functioning correctly. While some items have principle discriminations substantially different from zero, many items had principle discriminations near zero. These items do not contribute additional information about student understanding beyond a general understanding of electricity and magnetism.

The results for the principle discriminations, $a_{j k}$, were similar. Of the 47 discrimination parameters measured, 31 were significantly different between the samples (66\%), 21 were significantly different at the $p<0.001$ level (45\%); however, only 7 were different by more than $0.2(15 \%)$. Given the differences in student population and instructional environment, the measured discrimination parameters were somewhat similar suggesting the optimal models produced may be of general applicability.

The difference in overall difficulty for the two samples makes it challenging to interpret Table 3.7. To partially eliminate the effect of overall difficulty the items have been rank ordered from lowest difficulty and lowest overall discrimination to highest in Table 3.7. For most items the order of difficulty was generally similar; however, items 13, 19, 21, and 27 had difficulty ranks at least 10 positions higher in Sample 1 than in Sample 2 (they were much easier for students in Sample 1). Item 28 had a difficulty rank 10 positions higher in Sample 2. In general, the average absolute difference in difficulty rank was 4.7; if items $13,19,21,27$, and 28 are removed the average absolute difference falls to 3.2 indicating 
most items were fairly close to each other in rank; difficult questions in Sample 1 were also generally difficult in Sample 2. Only item 29 had a difference in overall discrimination rank of over 10. The average absolute difference in overall discrimination, $a_{0} j$, was 2.8 which fell to 2.4 if item 29 was eliminated.

It is likely that some of the differences in the discrimination parameters were a result of the overall difference in student performance for the two samples. While superficially independent in the MIRT model, Eqn. 3.6, the effective window on the difficulty produces correlations between difficulty and discrimination. Most difficulty parameters are between -3 and +3 ; very easy or very hard items have a limited range in which to discriminate between students. This effect can be quantified by calculating the correlation between the rank order of the difficulty and overall discrimination. For Sample 1, the correlation is $r=0.33$, a medium effect size, and for Sample $2 r=0.25$, a small to medium effect size.

This chapter replicated the method applied to the FCI in Chapter 3. As noted above, the optimal models for the CSEM contained more secondary principles (lemmas) than the FCI. Further, the principle discriminations, $a_{j k}$, were in general smaller than those found for the FCI. Only 7 principle discriminations were of magnitude 0.5 or greater in each of Sample 1 and Sample 2. For the FCI, 17 principle discriminations were greater than or equal to 0.5 , showing the FCI provides better resolution of the individual principles in its optimal model.

The selection of solution path 1 in models M1-2/M2-2 supports Leppävirta's identification of item 7 as a Newton's 3rd law item [113]. 


\subsection{Implications}

The optimal theoretical model presented in Table 3.3 is a very limited representation of the conceptual material covered by an introductory electricity and magnetism course. Many topics are missing or are weakly represented, such as the electric field of a point charge or the dipole nature of the magnetic field. Other topics are overrepresented such as the magnetic force on a stationary charge. Some items are difficult to interpret with experts producing substantially different solutions. Through the lens of the theoretical model in Table 3.3, the CSEM seems a weak instrument for a general evaluation of electricity and magnetism. The combination of an exhaustive theoretical model extending the model in Table 3.3 to include a more complete coverage of introductory electricity and magnetism and constrained MIRT may provide the appropriate framework for creating more robust and reliably interpreted instruments. The weak coverage could be identified by the expert solution analysis alone which can be performed during instrument development. MIRT provided confirmatory evidence that the expert model was a good model for student knowledge and allowed an optimal version of the theoretical model to be developed.

Ideally MIRT models of an instrument would have the same behavior across multiple samples; this was only partially supported by this study. The overall optimal models were very similar, differing in only a few principles. The difficulty was very different due to the substantial difference in performance of the two populations. Overall discriminations were also different, but principle discriminations were more similar. This suggests the parameters of the MIRT models are sensitive to student population and instructional environment and cannot be treated as universal. The structure of the optimal models were more general 
suggesting additional research will be able to identify a model that has acceptable fit for most institutions.

Ideally discriminations should be consistent across populations, assuming the instrument was developed with a sufficiently large and academically diverse population. Difficulty will vary with the overall performance of the different student samples. There is a relation between difficulty and discrimination that arises when items are either very high scoring or very low scoring because of windowing effects. If the items' average score is well away from these extremes, then discriminations should be consistent across populations because MIRT uses the relative difficulty of the items to set the scale for the ability traits, $\theta_{k}$. Topical coverage, however, could modify this relative difficulty ranking and produce differences in discrimination which may be the origin of the differences measured in this study.

Instructors can use the results in Table 3.7 to further understand CSEM results. Items that have a high principle discrimination are good measures of that principle. Items that have a single principle or only one principle with a high discrimination are particularly strong measures of that principle. For example, using Sample 1 and Table 3.7, items 3, 12, $13,16,17,18,21$, and 27 all depend on a single principle and have principle discriminations that are significantly different from zero. The item difficulty, $d_{j}$, for these items allow the comparison of student understanding for these principles; students understand L4, Coulomb's law for the electric force, (item $3, d_{3}=2.04$ ) and DF2, the definition of electric field, (item $12, d_{12}=2.66$ ) substantially better than C6, the relation of potential and field, (item 18 , $d_{18}=1.18$ ) and DF4, the definition of electric potential, (items 16 and $17, d_{16}=0.54$, $d_{17}=1.36$ ). Items such as item 1 which have multiple principles but discriminate more strongly on one principle can be used to measure understanding of that principle. Items 
which discriminate relatively equally on multiple principles may be used to characterize understanding of that combination of principles.

The large number of principles identified, 26, for a 32-item instrument meant that many principles were only included on small number of items and often mixed with other principles on the same item. This makes identifying what each individual item measures more challenging.

\subsection{Limitations}

This work compared two large samples from two institutions. Additional samples should be tested to determine if the results are general, particularly from institutions with different student demographics than the institutions studied.

The theoretical model presented in Table 4.5 was constructed from the solutions of a small set of expert practitioners. Other models are possible and should be explored. Most experts would agree on some segments of the model but there are other segments were multiple different models are possible. This should not be viewed as the end of the modelling process for the CSEM, but as the beginning. We feel constrained MIRT is the proper tool to explore alternate models.

\subsection{Conclusion}

Following the path set out by the FCI analysis, the CSEM MIRT analysis examined models of the CSEM for two large data sets drawn from different institutions. The optimal models identified were similar but not identical sharing 22 of the 26 principles included in 
either model. The optimal models had excellent model fit characteristics for both samples. Beyond the laws, definitions, facts, and corollaries needed to define the physics content of the instrument, both optimal models also contained additional qualitative principles derived from the more general principles. The overall difficulty and discrimination of the individual items were significantly different in most cases; however, the principle discriminations were more similar. The rank-ordered overall difficulties were also similar, but five items stood out as being relatively more difficult for the students in one of the samples. Therefore, while the models had many similarities, they were not identical; the optimal MIRT model for the CSEM does vary between institutions.

\subsection{MIRT Analysis Summary}

With both the FCI and the CSEM, MIRT was shown to be a productive technique for revealing the knowledge structure measured by the instrument. Using this technique two instruments with previously undefined granular structure were characterized. In both cases, several deficiencies were discovered affecting how these instruments should be used. Neither instrument includes complete coverage of the concepts included in a common college-level introductory course. Both instruments include many items which probe multiple overlapping concepts. This complexity makes it difficult for an instructor to determine which of these concepts are not well understood by the student. This confirms the unidimensional utility of these instruments and further shows that new instruments must be constructed if greater granularity of student knowledge is to be probed. For the sake of instructional utility, both instruments should be treated a unidimensional. This analysis shows the need to 
construct more advanced instruments moving forward. Multidimensional instruments that allow instructors to pinpoint student knowledge deficiencies are now possible using modern techniques like MIRT. 


\title{
Part II
}

\section{Using Machine Learning to Predict}

\author{
Course Grade
}


The following chapter represents a work submitted to Physical Review on a separate approach to improving outcomes in introductory physics courses. Unlike the previous part, where the focus was primarily on understanding the conceptual instruments used to evaluate the effectiveness of RBIS, Part II will focus on how machine learning can be applied to existing course data to construct early warning models of student failure. Chapter 5 includes the mostly unaltered version of this work submitted for publication. Parallel work on Physics 2 which was in the Supplemental Materials of the paper has been moved to the main text. Additionally, confusion matrices have been included for the benefit of the reader, should they wish to calculate any additional performance parameters. This work was conducted and submitted for publication in collaboration with Dr. John Stewart, Dr. Seth Devore, and Dr. Jie Yang. 


\section{Chapter 5}

\section{Using Machine Learning to Predict Course Grade}


This chapter examines the use of machine learning to predict physics class grades early in the semester. Most course instructors must wait until the first in-class exam to determine which students are in danger of failure. If course grade could be accurately predicted early in the class, it would allow instructors to intervene before students have developed poor course habits or done significant damage to their grade. This early intervention should help to improve course outcomes and keep students in the STEM pipeline, helping improve retention to graduation.

Improving STEM degree retention is not a new problem for educational research with many studies exploring this issue [121-128]. These studies have often found similar results showing measures of prior preparation, such as high school grade point overage (HSGPA) and ACT/SAT scores, coupled with student performance once arriving at college, measured by successful credit completion and college GPA (CGPA), produce statistically significant models of persistence.

Introductory physics courses along with introductory mathematics and chemistry courses form key early college hurdles for many STEM majors. While many factors affect the retention of students to STEM degrees, academic success in college classes must be viewed as of central importance to college completion. As such, promoting student success in core STEM courses may be one path to improving STEM retention. In the last 20 years, machine learning has been used to provide new insights into retention [129-133]. Machine learning techniques have only recently begun to be implemented in PER to understand the retention of physics students [134]. At the same time, the use of reformed instruction as an effective means of decreasing failure rates in STEM courses has grown [38]. Docktor and Mestre provide a thorough overview of the use of reformed instruction in PER in their 2014 synthesis of the 
field [3]. Studies of the effect of reformed instruction have usually focused on either course grade $[32,37,135]$ and/or student gains on conceptual instruments $[32,136-138]$ as measures of student success. This chapter develops models for the early semester identification of students at risk of receiving a grade of "C" or lower in introductory physics; these models could be used to direct reformed instructional interventions toward at-risk students, thus further improving student success and retention.

\subsection{Educational Data Mining}

Educational Data Mining (EDM) is a field which uses statistical, machine learning, and data mining techniques to understand large systems of educational data. Unlike data mining in other fields, such as business or genetics, EDM encompasses predictive modeling and integration with education research techniques such as psychometric modeling [133]. Romero and Venture [139] surveyed early work in EDM from 1995 to 2005 and found EDM studies primarily used relationship mining methods. By the time of Baker's review in 2009 [130], the majority of the works in EDM had shifted from relationship mining to prediction methods. In 2014, Peña-Ayala reviewed 240 EDM studies published between 2010 and 2013; $88 \%$ used probability, machine learning, and statistics as their analysis method [140]. Studies evaluating student performance, whether in-class or overall, comprised $21 \%$ of 240 studies and were the second most common type of study. The other categories of EDM identified in the Peña-Ayala review were student behavior modeling, assessment, student modeling, student support and feedback, curriculum, domain knowledge, sequencing, and teacher support. In addition to predicting student performance, EDM has also been used for other tasks such as 
analyzing student motivation, attitude and behavior, understanding student learning style, and improving E-learning and collaborative learning [132]. The growth in EDM has lead to many universities adopting systems utilizing these methods to improve their course outcomes and in-term retention of STEM students [141].

A number of attempts have been made to classify the methods used in EDM. Romero and Ventura [139] used two broad categories: web mining (including clustering, classification, text mining, relationship mining), and statistics and validation. In 2008, Romero et al. [142] identified logistic regression, decision trees, random forests, neural networks, naive Bayes, support vector machines, and K-nearest neighbor algorithms as the most commonly applied EDM methods. In 2009, Baker produced a more complex EDM taxonomy in 5 parts: Prediction (classification, regression, density estimation), Clustering, Relationship Mining, Distillation of Data for Human Judgment, and Discovery with Models [130]. By 2014, EDM had grown to the extent that Peña-Ayala [140] classified the field under multiple, somewhat overlapping, categories focused on the models used (descriptive vs. predictive), tasks (clustering, regression, ...), methods (decision trees, Bayes theorem...), techniques (logistic regression, hierarchical clustering, ...), and algorithms (K-means, naive Bayes, ...). Classification was used in $42 \%$ of the studies while either clustering or regression were used in an additional $42 \%$ of studies. Decision trees and logistic regression were used in $18 \%$ of works with only Bayes theorem analysis employed more frequently in $20 \%$ of the studies. A review by Shahiri et al. of the prediction of students' academic performance using data mining techniques [143] compared 5 major algorithms applied in 30 studies published between 2002 and 2015: decision trees, neural networks, naive Bayes, K-nearest neighbors, and support vector machines. Neural networks and decision trees were the most commonly 
used techniques. In both reviews, most studies focused on overall academic performance and not on course-level performance.

The methods used in the current study, decision trees, random forests (a method using many decision trees), and logistic regression, will be discussed in detail in Section 5.4. Additional information on other machine learning techniques may be found in a number of machine learning texts $[144,145]$.

\subsection{EDM and Grade Prediction}

There have been several studies in EDM which produced models that predicted student grades in undergraduate courses. Huang and Fang [146] used linear regression, multilayer perceptron network modeling, radial basis function network modeling, and support vector machines to predict performance on the final exam in a high-enrollment core engineering course. This study used CGPA, performance in 4 prerequisite courses (Statics, Calculus 1, Calculus 2, and Physics 1), and scores on the three in-semester exams as independent variables and found minimal differences in the accuracy of models constructed using different algorithms. They recommended using Ordinary Least Squares (OLS) regression with only CGPA as an independent variable to predict average class performance. However, for individual student grade prediction, they found support vector machines with CGPA, all four prerequisite course grades, and the results of the first in-class examination as independent variables produced the best model. Marbouti, Diefes-Dux, and Madhavan [147] built predictive models with variables measured in the class using six different algorithms: logistic regression, support vector machines, decision trees, multilayer perceptron networks, naive Bayes, K- 
nearest neighbors, and a final ensemble model consisting of the three most successful individual models. Their study predicted course performance at week 5 of the semester where homework, quiz, and test 1 scores were available; defined success as earning a grade of "C" or better; and studied a first-year engineering course. They found logistic regression and an ensemble model combining support vector machines, K-nearest neighbors, and naive Bayes to be superior with a prediction accuracy of $94 \%$ for logistic regression and $92 \%$ for the ensemble model. In 2010, Macfadyen and Dawson [148] mined data from the course Learning Management System (LMS) of an undergraduate biology course to identify 15 variables with significant correlation to final grade. Logistic regression models predicted students at risk of failure (final grade of less than $60 \%$ ) with $70 \%$ accuracy and correctly identified students that failed the course (final grade less than 50\%) with $81 \%$ accuracy.

Outside of STEM, Hu, Lo, and Shih [149] used classification and regression trees with data from a LMS to develop an early warning system for students in an undergraduate information literacy and ethics course. Using this method, they achieved accuracies above 97\%; however, they had to re-sample the data to overcome a significant class imbalance (less than $6 \%$ of the students failed the class). In 2004, Ballard and Johnson [150] examined mathematics skills using multiple variables: ACT mathematics percentile, if a student had already taken calculus, if a student was required to take remedial mathematics, and scores on a basic mathematics concept test to predict course grade in an introductory microeconomics course. This study of 1462 individual students used institutional data combined with in-class data and found that a student's CGPA along with multiple measures of quantitative skills were necessary to construct optimal models of student performance. A 2013 study by bin Mat et al. [141] of the adoption of EDM methods by educational practitioners recommended 
the use of neural networks and decision trees with attendance and prerequisite course grades as independent variables.

A substantial body of EDM research has examined online courses. A 2012 study [151] of 1144 students across 138 online courses used cluster analysis to determine question themes and demonstrated that those themes were significant predictors of course performance using a logistic regression model. In a separate study investigating online business course

success [152], CGPA, attendance at orientation, reading scores, number of previous course withdrawals, age, number of previous online courses taken, and ACT English scores were found to be strongly correlated with course performance. A linear regression analysis, however, only identified attendance at orientation and CGPA as significant predictors of course performance despite the large number of correlates.

\subsection{Research Questions}

This study seeks to answer the following research questions:

RQ1: How well can introductory physics course grades be predicted early in the semester?

RQ2: What variables are most important for the accurate prediction of physics course grades early in the semester?

\subsection{Methods}

\subsubsection{Context for Research}

This study was performed in the introductory physics classes at a large eastern landgrant university serving approximately 30,000 students. The undergraduate population had 
ACT scores ranging from 21 to 26 (25th to 75 th percentile) [93]. The overall undergraduate demographics were $79 \%$ White, $7 \%$ international, $4 \%$ African American, $4 \%$ Hispanic, $4 \%$ students reporting with two or more races, and other groups each with $1 \%$ or less. The sample was primarily male (81\%) [93].

Data were collected from both Physics 1 (introductory calculus-based mechanics) and Physics 2 (introductory calculus-based electricity and magnetism). These courses are required for most physical science and engineering majors at the institution. The structure of the courses was similar, but not identical, for the period studied. Each course was led by a single experienced instructor who implemented research-based instructional practices for the period studied. The courses met for three 50-minutes lectures and one 3-hour laboratory each week. Each lecture used Peer Instruction with clickers [21] to engage students in conceptual learning; the grades for the student's clicker responses were based on participation and are called Lecture Quiz grades (accounting for 5\% of the Physics 1 grade and $4 \%$ of Physics 2 grade). Lab sessions featured a mixture of inquiry-based hands-on activities, conceptual white-boarding activities, group problem solving, and traditional experiments. Students received a grade for completing the laboratory (LabGrade) and also completed a graded quiz (LabQuiz). Lab and Lab Quiz grades disaggregated by class and week were only available in Physics 2 and represented $5 \%$ of the course grade each. Both classes assigned homework each week which was a mix of conceptual and quantitative problems and was graded to provide the variable HwkGrade. Physics 1 used an online homework system that assigned problems from a popular textbook and allowed multiple attempts for each problem and accounted for $15 \%$ of the course grade. Physics 2 assigned problems to be worked on paper which were graded by teaching assistants; the problems were written specifically for the class and accounted 
for $7 \%$ of the course grade. All grade variables were cumulative; for example, the week 4 homework grade was the student's average homework grade in the first 4 weeks of class. To measure changes in conceptual understanding, Physics 1 administered the Force and Motion Conceptual Evaluation (FMCE) [153] as a pretest and post-test; Physics 2 administered the Conceptual Survey of Electricity and Magnetism (CSEM) [16] as a pretest and post-test. Only the pretest scores were used in this study. Each in-class test $13 \%$ of the course grade in Physics 1 and 25\% in Physics 2.

\subsubsection{Sample}

The Physics 1 sample was collected over four semesters from fall 2015 to spring 2017 in which time 1588 students enrolled in the course. For students taking the course more than once, only their final attempt was retained. Any records with missing data were also removed leaving 915 complete records. The course was team taught by the same two instructors during the time the data was collected and no significant changes to instruction were made during this period. The Physics 2 sample was collected from fall 2015 to spring 2017 semesters in which time 1282 students enrolled in the course. Duplicate students and those with missing data were removed from the data set leaving 805 complete records. Most students were removed for either missing pretest scores, missing HSGPA, or missing ACT/SAT scores.

\subsubsection{Variables}

The variables used in this study were drawn from institutional records and from variables collected within the physics classes and are summarized in Table 5.1. The inclass variables were described in Sec. 5.4.1. The institutional variables are defined in Table 
Table 5.1: Full list of both institutional and in-class variables. An " $\mathrm{X}$ " in either the Physics 1 or Physics 2 columns denotes that the variable was available for that data set. True is abbreviated T, false, F. In the variable MathEntry, Algrebra encompasses college algebra and all lower course, pre-calculus any course between college algebra and calculus 1 , and calculus as any course at or above calculus 1 .

\begin{tabular}{|c|c|c|c|}
\hline \multicolumn{4}{|r|}{ Institutional Variables } \\
\hline Variable & Physics 1 & Physics 2 & Description \\
\hline Gender & $\mathrm{X}$ & $\mathrm{X}$ & Gender $($ Men $=1$ Women $=0)$. \\
\hline InState & $\mathrm{X}$ & $\mathrm{X}$ & Student is resident of the state where the institution is located $(\mathrm{T}=1, \mathrm{~F}=0)$. \\
\hline URM & $\mathrm{X}$ & $\mathrm{X}$ & Student does not identify as White non-Hispanic $(\mathrm{T}=1, \mathrm{~F}=0)$ \\
\hline MathEntry & $\mathrm{X}$ & $\mathrm{X}$ & First math class taken (Calculus,Pre-Calculus, and Algebra). \\
\hline FirstFall & $\mathrm{X}$ & $\mathrm{X}$ & Started in a fall semester $(\mathrm{T}=1, \mathrm{~F}=0)$ \\
\hline FirstGen & $\mathrm{X}$ & $\mathrm{X}$ & Student is a first generation college student $(\mathrm{T}=1, \mathrm{~F}=0)$. \\
\hline CmpPct & $\mathrm{X}$ & $\mathrm{X}$ & Percentage of hours attempted that were completed at the start of course. \\
\hline CGPA & $\mathrm{X}$ & $\mathrm{X}$ & College GPA at start of course. \\
\hline STEMHrs & $\mathrm{X}$ & $\mathrm{X}$ & $\begin{array}{l}\text { Number of STEM (Math, Bio, Chem, Eng, Phys) } \\
\text { credit hours completed at start of course. }\end{array}$ \\
\hline HrsCmp & $\mathrm{X}$ & $\mathrm{X}$ & Total credits hours earned at start of course. \\
\hline HrsEnroll & $\mathrm{X}$ & $\mathrm{X}$ & Total credits hours enrolled at start of course. \\
\hline P1Grade & & $\mathrm{X}$ & (Dependent Variable) Grade for last Physics 1 attempt (A or $\mathrm{B}=1$, CDFW $=0$ ). \\
\hline P1Atmp & & $\mathrm{X}$ & Physics 1 attempted more than once $(\mathrm{T}=1, \mathrm{~F}=0)$ \\
\hline P2Grade & & $\mathrm{X}$ & (Dependent Variable) Grade for last Physics 2 attempt (A or $\mathrm{B}=1, \mathrm{CDFW}=0$ ). \\
\hline HSGPA & $\mathrm{X}$ & $\mathrm{X}$ & High school GPA. \\
\hline ACTM & $\mathrm{X}$ & $\mathrm{X}$ & ACT/SAT mathematics percentile. \\
\hline ACTV & $\mathrm{X}$ & $\mathrm{X}$ & ACT/SAT verbal percentile. \\
\hline Cal1Grade & $\mathrm{X}$ & $\mathrm{X}$ & Grade for last Calculus 1 attempt ( $\mathrm{A}$ or $\mathrm{B}=1$, CDFW $=0$ ). \\
\hline Cal1Atmp & $\mathrm{X}$ & $\mathrm{X}$ & Calculus 1 was attempted more than once $(\mathrm{T}=1, \mathrm{~F}=0)$ \\
\hline APCount & $\mathrm{X}$ & $\mathrm{X}$ & Number of courses where AP credit was received. \\
\hline APCredit & $\mathrm{X}$ & $\mathrm{X}$ & Number of credits hours received for AP tests. \\
\hline TransCnt & $\mathrm{X}$ & $\mathrm{X}$ & Number of courses where transfer credit was received. \\
\hline TransHrs & $\mathrm{X}$ & $\mathrm{X}$ & Number of credits hours received for transfer courses. \\
\hline & & & In-Class Variables \\
\hline Variable & Physics 1 & Physics 2 & Description \\
\hline FMCEPre & $\mathrm{X}$ & & Percentage score on the FMCE pretest. \\
\hline Test 1 & $\mathrm{X}$ & $\mathrm{X}$ & Percentage score on the first exam of the semester. \\
\hline CSEMPre & & $\mathrm{X}$ & Percentage score on the CSEM pretest. \\
\hline LecQuiz & $\mathrm{X}$ & $\mathrm{X}$ & Average grade on the lecture quiz by each week. \\
\hline LabQuiz & & $\mathrm{X}$ & Average grade on the lab quizzes by each week. \\
\hline HwkGrade & $\mathrm{X}$ & $\mathrm{X}$ & Average grade on the homework by each week. \\
\hline LabGrade & & $\mathrm{X}$ & Average grade for the laboratory by each week. \\
\hline
\end{tabular}


5.1. A few variables require additional explanation. The variable MathEntry measures the first mathematics class the student enrolled in at the institution. It has three levels: "Calculus" for students who first enrolled in Calculus 1 or a more advanced mathematics class, "Algebra" for students who first enrolled in College Algebra, and "Pre-Calculus" for students who first enrolled in a class between College Algebra and Calculus 1. The variable STEMHrs captures the number of credit hours of STEM classes completed before the start of the course modeled. STEM classes include mathematics, biology, chemistry, engineering, and physics classes.

\subsubsection{Classification Models}

Classification models attempt to predict categorical outcomes. This study predicts the dichotomous outcomes "P1Grade" and "P2Grade" where students who received an "A" or "B" in Physics 1 were coded as P1Grade $=1$ while student who received a lower grade were coded as P1Grade $=0$. Similar coding was used for Physics 2 to produce P2Grade. The models constructed "classify" students into one of these two categories for each course. For example, the logistic regression classifier predicts the probability that a student will be measured with $\mathrm{P} 1$ Grade $=1$. If this probability is greater than 0.5 , then the classification model assigns that student to the class P1Grade $=1$ otherwise the student is assigned to the class $\mathrm{P} 1$ Grade $=0$.

To construct a classification model, the full data set is split into two subsets: the training and test data sets. This is done by randomly sampling the full data set without replacement. The training data set is used to construct or "train" the models, while the test data set is reserved for the purpose of evaluating the model performance when classifying 
"new" data. As much data as possible should be allocated to the training data set to ensure the creation of the most accurate model while retaining sufficient data in the test data set for accurate characterization of model performance. For this work, $62 \%$ of the data was allocated to the training data set and $38 \%$ to the test data set; splits as low as $50 \%$ test, $50 \%$ training have been shown to provide accurate results [154].

After a model is constructed, it is then used to make predictions of the outcomes of the test data producing a matrix containing the frequency of prediction outcomes called the confusion matrix [155] as shown in Table 5.2. The confusion matrix compares the predicted outcome to the actual outcome for the test data set. The on-diagonal terms represent correct predictions and off-diagonal terms incorrect predictions. The sum of the entries in the confusion matrix is the size of the test data set, $N_{\text {test }}$.

Many different statistics characterizing prediction accuracy can be computed from the confusion matrix; in this work model accuracy, the fraction of correct predictions, Eqn. 5.1, is reported.

$$
\text { accuracy }=\frac{\text { True Neg. }+ \text { True Pos. }}{N_{\text {test }}}
$$

Where $N_{t} e s t$ is the total number of individuals in the test data.

Table 5.2: Confusion Matrix Example

\begin{tabular}{c|c|c} 
& Actual Negative & Actual Positive \\
\hline Predicted Negative & True Negative & False Negative \\
\hline Predicted Positive & False Positive & True Positive
\end{tabular}

Accuracy can be misleading because models with substantial accuracy can be constructed by pure guessing. For example, if the sample has an outcome equally balanced between two classes, a classification model which assigns all individuals to the same class will have an 
accuracy of $50 \%$. To compensate for this effect, Cohen's kappa was developed to provide a measure of accuracy normalized to the baseline accuracy of random chance [43]. Cohen's kappa is shown in Eqn 5.2,

$$
\kappa=\frac{p_{0}-p_{e}}{1-p_{e}}=1-\frac{1-p_{0}}{1-p_{e}}
$$

where $p_{0}$ is the model accuracy and $p_{e}$ is the probability of randomly guessing the correct classification. Model fit is classified as follows: less than 0.2 as poor agreement, 0.2 to 0.4 as fair agreement, 0.4 to 0.6 as moderate agreement, 0.6 to 0.8 as good agreement, and 0.8 to 1.0 as excellent agreement [156].

The final method of characterizing the quality of the models constructed in this paper is the Receive Operating Characteristic (ROC) Curve, a technique originally developed to determine if radar receivers were accurately detecting aircraft. The ROC curve is constructed by plotting the true positive rate (sensitivity) against the false positive (1-specificity) rate for all values of the decision threshold from 0 to 1 as can be seen in Fig. 5.1, which shows the ROC curve for the Physics 2 logistic regression model of the institutional variables. Each classification model produces a value for the probability of each outcome. The decision threshold is the probability where a particular outcome would be selected; it is not always optimal to select at a threshold of 0.5 . The ROC curve provides a measure of the model's discrimination between the outcomes as measured by the Area Under the Curve (AUC). In a model that is no better than guessing, the AUC will be 0.5 and the ROC curve will be a straight line. A model with perfect discrimination characteristics would have an AUC of 1.0 [155, 157]. Hosmer et al. [157] suggest an AUC threshold of 0.80 for excellent discrimination. 


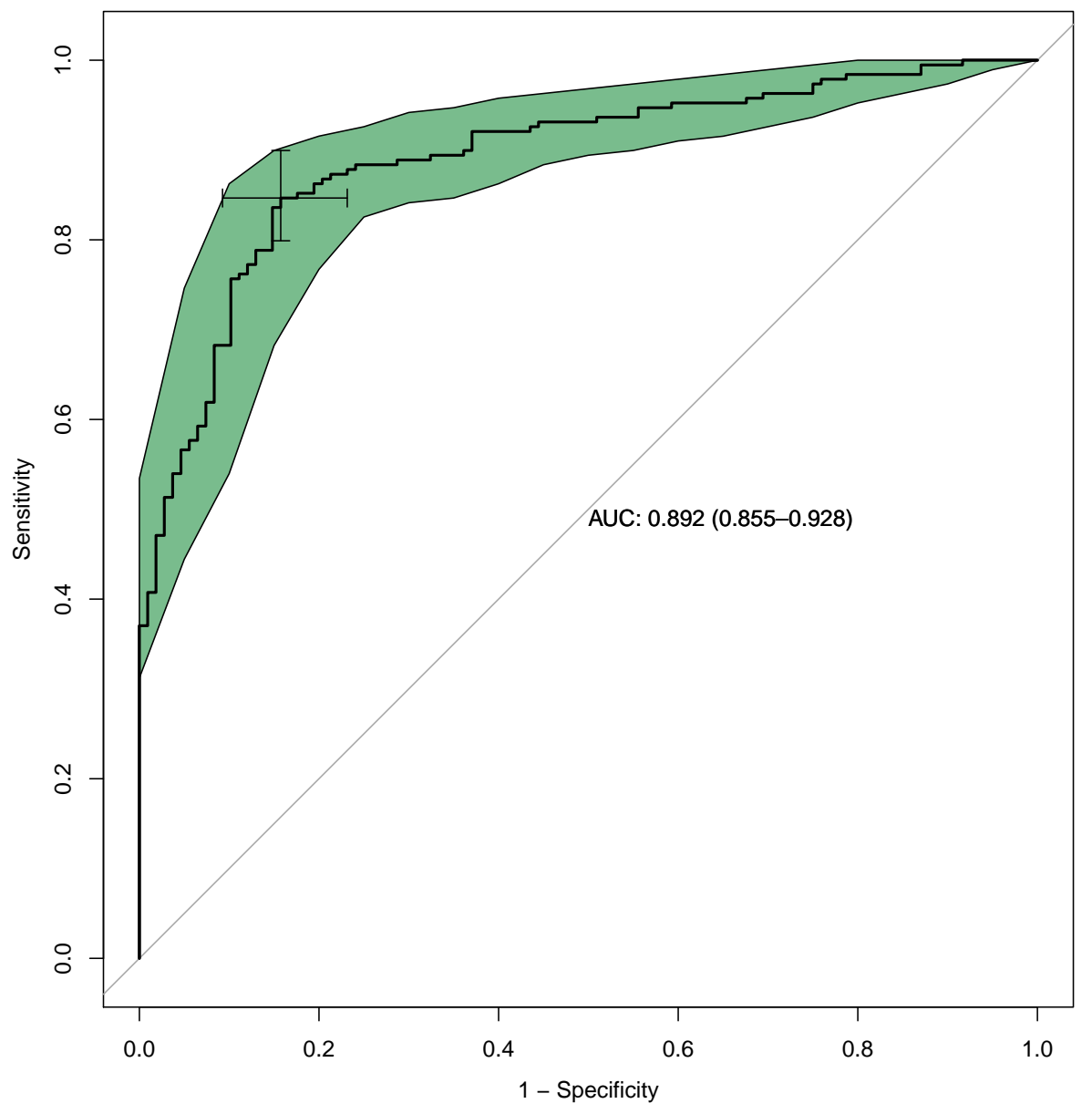

Figure 5.1: ROC Curve Example with 95\% CI. The x-axis represents the false positive rate with specificity defined as the true negative rate, $T N /(T N+F P)$. The y-axis represents the true positive rate or sensitivity, $T P /(T P+F N)$. AUC is the area under the ROC curve and is analogous to accuracy. A ROC curve for a perfectly fit model will form a right angle at the upper left corner and have and AUC of 1.0 while a model that is no better than guessing will be collinear with the line in the middle of the plot and have an AUC of 0.50 . 
A baseline model was created for each sample by predicting all students in the test data set would have the most common outcome in the training data set. For example, in Physics $163 \%$ of the students received an "A" or "B," the baseline model classifies all students as students who will receive an "A" or "B" producing a classification that is $63 \%$ accurate, but represents pure guessing.

\subsubsection{Classification Methods}

Two statistical techniques were used in the prediction of student grades in this paper: logistic regression and random forests. Several different classification methods were examined: logistic regression, K-nearest neighbors, classification and regression trees, naive Bayes, support vector machines, and random forests. Logistic regression and random forests were ultimately selected. Logistic regression was often selected as the best model in the literature and random forests represent one of the most commonly used techniques in EDM; each has its own unique advantages and disadvantages which complement each other. These will become evident in the following sections.

\section{Logistic Regression}

Logistic regression represents one of the most widely used classification methods. In logistic regression, the probability $P(Y=1)$ of a binary dependent variable $Y=0,1$ is predicated by a set of independent variables $X_{i}$. The probability, which is restricted to the range $[0,1]$, is first projected on the range $[0, \infty]$ by calculating the odds; odds $=P /(1-P)$. The odds are projected into an unbounded range by taking the logarithm. Logistic regression, then, employs methods related to ordinary linear regression to minimize the error in Eqn. 
5.3 (below) by selecting an optimal set of regression coefficients, $\beta_{i}$.

$$
\ln \left(\frac{P(Y=1)}{1-P(Y=1)}\right)=\beta_{0}+\sum_{i=1}^{k} \beta_{i} \cdot X_{i}
$$

where $k$ is the number of independent predictor variables. The odds of $Y=1$ is found by exponentiating Eqn. 5.3. If all other variables are constant, a unit increase in $X_{1}$ multiplies the odds by $e^{\beta_{1}}$, the odds ratio. We report the odds ratio instead of the regression coefficient, $\beta_{i}$. The underlying statistical assumptions of logistic regression are different than Ordinary Least Squares and models are estimated using Maximum Likelihood techniques.

The odds ratio for each coefficient multiplies the base odds, the odds when all coefficients except the intercept are zero. If the odds ratio is above one, then the odds ratio minus one multiplied by 100 is equal to the percentage increase in the odds. For example, if the odds ratio $e^{\beta_{1}}=1.4$ then an increase in $X_{1}$ by one unit increases the odds of receiving an "A" or "B" by 40\%. If the odds ratio is less than one, the odds ratio is inverted before subtracting 1 and multiplying by 100 to yield the percentage decrease in odds. For example, if $e^{\beta_{1}}=0.25$, then an increase in $X_{1}$ of one unit decreases the odds of receiving an "A" or "B" by $(1 / 0.25-1) \cdot 100 \%=300 \%[144]$.

Logistic regression requires certain assumptions to be met to produce valid results. The dependent variable or outcome must follow a binomial distribution [157]. Outcomes must also be statistically independent and the continuous independent variables must be related linearly to the log odds. Logistic regression is not robust against collinearity in the independent variables and as with linear regression, models with high multicollinearity may be problematic. 
For each logistic regression model or logistic model, each variable was fit independently in a univariate logistic model. The fits for each were evaluated with a very liberal screening criterion, retaining variables with $p$ value of 0.20 to 0.25 to filter out the least important variables. Once the variables meeting the screening criterion were selected, a logistic model was constructed using all the selected variables and then pruned step-wise to the most parsimonious model by retaining variables with $p<0.05$ [157]. This model is called the "Optimal" model in the present work. Results were then confirmed using a separate backwards step-wise process that minimized the AIC of the model using the "stepAIC" function from the "R" "MASS" package [158], which step-wise removes parameters from the model until the removal of parameters no longer significantly decreases the AIC.

For the logistic modeling of the weekly data, the in-class only model was fit first. Once the optimal in-class model was found, the institutional variables selected in the optimal institutional logistic model were then added to these models and the pruning process was performed again to select the best fitting combined model.

\section{Decision Trees}

In order to understand random forests, it is first necessary to understand the decision trees upon which they are built. Decision trees are a machine learning algorithm which splits the data set into two or more "most" homogeneous groups based on the measured variables. The algorithm works by taking the data set, splitting it by each of the independent variables, and then measuring the degree to which these splits have created subsets of data which are maximally homogenous by outcome (each split should contain as large a percentage of one outcome as possible). Each split subset is then split using the same criteria producing a 
tree where each node is characterized by the criteria used to make the split (the decisions). If allowed, this algorithm will continue to split the data until each terminal node or leaf is perfectly homogenous (contains only one of the possible outcomes). To ensure that the model is not overfit, decision trees are "pruned" back to a model that balances the complexity of the model with the predictive power of the model. Because the models are based solely on homogenizing outcomes, decision trees are not susceptible to multicollinearity [159].

\section{Random Forests}

Random forests are an extension of the decision tree algorithm where, instead of a growing single tree for the model, thousands of trees are grown. This "forest" of decision trees is used to fit the data and then the ensemble "votes" for the most likely outcome. Each decision tree is used to classify each participant (an individual student in our case) and the most commonly occurring classification of the forest is selected. Random forests are a bootstrapping technique where the individual trees are grown on $Z$ samples of size $N$ generated by sampling the training data set with replacement. Each of these $Z$ unique samples is fit with a decision tree which uses a subset $m=\sqrt{k}$ of the available variables

$k$ [160]. Using a subset of the available parameters accomplishes two goals: first, the trees are decorrelated from each other, and second, the strongest predictors are prevented from always overwhelming some of the weaker predictors. With the exception of these differences, the trees are grown using the same technique as in the previous section without pruning [144]. Random forests also provide a characterization of the relative importance of the independent variables through a number of variable importance indices which indicates the degree to which each variable is influential in the model. The "randomForest" package in 
"R" produces two commonly used versions of variable importance: mean decrease in Gini index and mean decrease in accuracy [161]. This paper reports the more intuitive mean decrease in accuracy. The mean decrease in accuracy is determined as the average decrease in accuracy across all trees if the variable was removed [160].

In order to construct the optimal random forest models, 10,000 decision trees were constructed using all available variables. The 1-SE rule [159] was then applied to select the optimal random forest model. This was accomplished by fitting the ROC curve for the full model, then removing variables and selecting the most parsimonious model whose ROC curve was within 1-SE, 1 standard error, of the full model.

For the random forest analysis in this work, the optimal in-class model was found and the variables from the institutional random forest model were added; however, no pruning was done due to the limited number of parameters in the model.

\subsubsection{Opening the "Black Box" of Machine Learning: Local Interpretable Model- Agnostic Explanations}

While machine learning provides powerful tools for understanding large data sets, the algorithms work as "black boxes" where the model builds itself. Even if the model fits well and predicts the test data set adequately, it is difficult to extract additional meaning from the predictions. Ribeiro, Singh, and Guestrin [162] developed a method for explaining how any machine learning model makes its predictions by assuming that locally all models behave linearly. Their Local Interpretable Model-Agnostic Explanations (LIME) algorithm works by selecting the record of a single student and fitting a sparse linear model to predict

that one outcome. The process then perturbs the model around that fit and uses data 
from other students close by to determine the degree to which each variable is necessary to the predicted outcome. Using this method, it is possible to see which variables influence a model's decision and the degree of importance these variables have to that decision. This can be used to determine if a model is making decisions based upon variables that make sense (for example, a student with a high CGPA is more likely to graduate) rather than spurious correlations (for example, not attending class increases the odds of course success).

The LIME algorithm can also be used diagnostically to determine the features of an individual student's performance which are most predictive of his or her success or failure.

\subsection{Results}

The goal of this work was to understand the variables important to the prediction of physics course grades at six time points early in two introductory physics classes: before the class begins using only institutional data and at the end of weeks 1 through 5 . The results for Physics 1 and 2 are discussed separately.

\subsubsection{Physics 1 Model Performance}

As the Physics 1 class progresses and the students complete assignments, the classification models become more accurate. Table 5.3 reports the accuracy, $\kappa$, and AUC for the in-class models and the combined institutional/in-class models for week 1 through week 5 as well as the institutional model using data available before students begin the course. For the institutional model, both results using all variables and results pruning the model to an optimal model are presented. Figure 5.2 plots the evolution of these quantities for the weekly models. The random guessing baseline model is also presented; the horizontal axis 

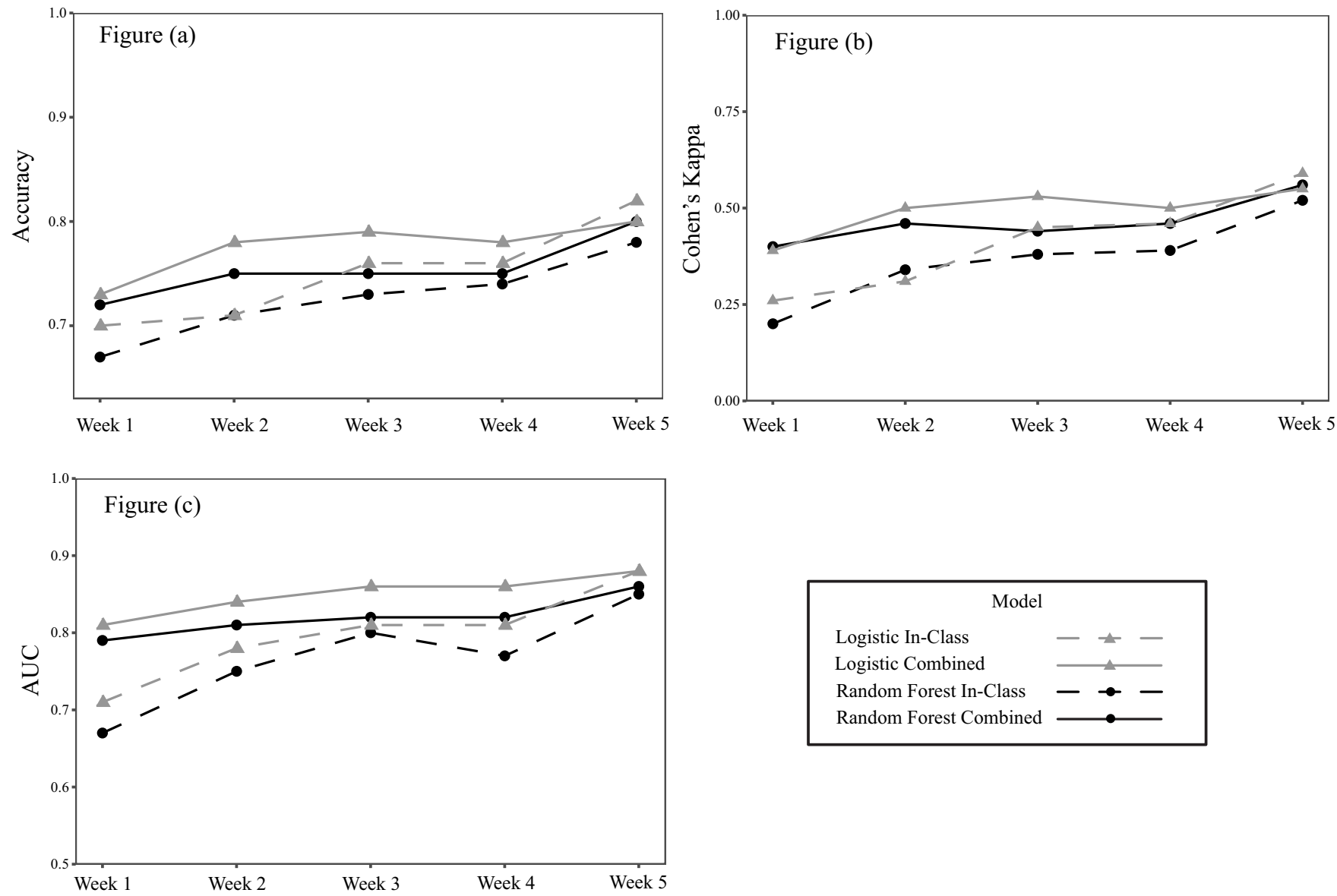

Figure 5.2: Physics 1: Model accuracy (Fig. (a)), Cohen's kappa (Fig. (b)), AUC (Fig. (c)). The horizontal axis represents the performance of the baseline model in each plot. 


\begin{tabular}{c|c||c|c|c|c||c|c|c}
\multicolumn{4}{c|}{} & \multicolumn{4}{c||}{ Logistic Regression } & \multicolumn{4}{c}{ Random Forests } \\
\hline Model & Variables & Accuracy & $\kappa$ & AUC & $R^{2}$ & Accuracy & $\kappa$ & AUC \\
\hline Baseline & None & 0.63 & 0.00 & 0.50 & & 0.63 & 0.00 & 0.50 \\
\hline \multirow{2}{*}{ Institutional } & All & 0.71 & 0.35 & $0.78[0.72,0.83]$ & 0.35 & 0.73 & 0.40 & $0.78[0.73,0.83]$ \\
& Optimal & 0.70 & 0.32 & $0.79[0.74,0.84]$ & 0.33 & 0.72 & 0.40 & $0.77[0.71,0.82]$ \\
\hline \multirow{2}{*}{ Week 1 } & In-Class & 0.70 & 0.26 & $0.71[0.65,0.76]$ & 0.16 & 0.67 & 0.20 & $0.67[0.61,0.73]$ \\
& In-Class/Institutional & 0.73 & 0.39 & $0.81[0.77,0.86]$ & 0.37 & 0.72 & 0.40 & $0.79[0.75,0.84]$ \\
\hline \multirow{2}{*}{ Week 2 } & In-Class & 0.71 & 0.31 & $0.78[0.72,0.83]$ & 0.24 & 0.71 & 0.34 & $0.75[0.69,0.80]$ \\
& In-Class/Institutional & 0.78 & 0.50 & $0.84[0.80,0.89]$ & 0.41 & 0.75 & 0.46 & $0.81[0.76,0.86]$ \\
\hline \multirow{2}{*}{ Week 3 } & In-Class & 0.76 & 0.45 & $0.81[0.76,0.86]$ & 0.30 & 0.73 & 0.38 & $0.80[0.75,0.85]$ \\
& In-Class/Institutional & 0.79 & 0.53 & $0.86[0.81,0.90]$ & 0.43 & 0.75 & 0.44 & $0.82[0.78,0.87]$ \\
\hline \multirow{2}{*}{ Week 4 } & In-Class & 0.76 & 0.46 & $0.81[0.77,0.86]$ & 0.32 & 0.74 & 0.39 & $0.77[0.72,0.83]$ \\
& In-Class/Institutional & 0.78 & 0.50 & $0.86[0.81,0.90]$ & 0.43 & 0.75 & 0.46 & $0.82[0.78,0.87]$ \\
\hline \multirow{2}{*}{ Week 5 } & In-Class & 0.82 & 0.59 & $0.88[0.85,0.92]$ & 0.46 & 0.78 & 0.52 & $0.85[0.81,0.89]$ \\
& In-Class/Institutional & 0.80 & 0.55 & $0.88[0.84,0.92]$ & 0.51 & 0.80 & 0.56 & $0.86[0.82,0.90]$ \\
\hline
\end{tabular}

Table 5.3: Physics 1: Logistic and Random Forest model performance. The 95\% confidence interval for AUC is shown in brackets.

represents the performance of the baseline model in each plot in Fig. 5.2.

Using logistic regression, the third week of the semester was the first where the models using only in-class variables outperformed the model using only the institutional variables with superior $\kappa$ and AUC. We will focus on $\kappa$ and AUC because, unlike accuracy, these two measures correct for the effect of guessing. In weeks 1 through 4 , the combined inclass/institutional models outperformed each of the in-class only models for that week; however, this was not the case in week 5 . The week 5 logistic models were statistically indistinguishable using DeLong's test comparing the AUC of the separate ROC curves, indicting that by the first in-semester examination the institutional variables were no longer necessary for the prediction of student grades. The weekly logistic models demonstrate the importance of including the institutional data if student risk is to be accessed very early in the semester.

The random forest results in Table 5.3 show that the random forest models performed almost identically to the logistic models. Unlike the logistic models, the $\kappa$ of the in-class-only random forest model only exceeded the optimal institutional model in week 5 . The optimal 
in-class-only model AUC exceeded the institutional model AUC in week 3, but the two AUC values were equal in week 4 . In week 5 , the in-class-only model once again was no longer distinguishable from the combined institutional/in-class model.

\subsubsection{Physics 1 Variable Importance}

\section{Institutional Model}

For Physics 1, the optimal logistic model of the institutional data required only a small subset of the available variables, as shown in Table 5.4. This optimal model had acceptable, but average, fit statistics with an accuracy of $70 \%$, an improvement of $7 \%$ over the baseline model, and Cohen's kappa of 0.32 representing fair agreement of the model with the test data set [156]. The ROC curve produced an AUC of 0.79 , with a $95 \%$ confidence interval that included the 0.80 threshold for excellent discrimination. Each logistic model reports the odds-ratio and its $95 \%$ confidence interval using unstandardized variables. For example,

in Table 5.4 a one-point increase in CGPA on a four-point scale multiplies the odds by 11.4. The normalized odds ratio uses standardized continuous variables. To standardize or normalize a variable, the mean is subtracted from the variable and the result is divided by the standard deviation. A one-standard-deviation increase in CGPA multiplies the odds by 3.47 , an increase in odds of $247 \%$. Each model also reports an intercept; this is the base odds if all the independent variables are zero. Most unnormalized intercepts are zero; a student with a zero CGPA or HwkGrade has a very small chance of passing the class. The normalized intercept represents the odds of a student with all dichotomous variables equal to zero and average values on all continuous variables of receiving an "A" or "B" in the course. 
Table 5.4: Physics 1: Optimal institutional logistic model. The z-score is the test statistic used to determine if the odds ratio is statistically significant.

\begin{tabular}{c|c|c|c|c|c}
\hline Variable & $\begin{array}{c}\text { Odds } \\
\text { Ratio }\end{array}$ & $95 \%$ CI & $\begin{array}{c}\text { Norm } \\
\text { Odds } \\
\text { Ratio }\end{array}$ & Z-score & $p$-value \\
\hline Intercept & 0.00 & {$[0.00,0.00]$} & 4.93 & -8.69 & $<0.001$ \\
CmpPct & 1.03 & {$[1.02,1.05]$} & 1.68 & 3.90 & $<0.001$ \\
CGPA & 11.40 & {$[6.62,19.64]$} & 3.47 & 8.77 & $<0.001$ \\
ACTM & 1.02 & {$[1.01,1.04]$} & 1.45 & 2.64 & 0.008 \\
MathEntry: & 0.27 & {$[0.13,0.53]$} & 0.27 & -3.73 & $<0.001$ \\
Algebra & & {$[0.16,0.52]$} & 0.29 & -4.15 & $<0.001$ \\
$\begin{array}{c}\text { MathEntry: } \\
\text { PreCal }\end{array}$ & 0.29 & {$[0.15$} & &
\end{tabular}

The optimal institutional logistic model required four variables: CGPA, ACTM, CmpPct, and MathEntry. Higher CGPA, ACTM, and CmpPct increased the odds of a student receiving an "A" or "B" in the course. Math Entry Point is a 3-level categorical variable with levels: Calculus, Pre-Calculus, and Algebra where Calculus was used as the reference level for the variable. Students who did not arrive at the university ready to take calculus had lower odds of receiving an $\mathrm{A} / \mathrm{B}$ in Physics 1. CGPA was much more important than either CmpPct or ACTM with an increase in CGPA of one standard deviation corresponding to an increased odds of receiving an A/B of $247 \%$. Students starting mathematics in a course below Calculus 1 had significantly lower odds of earning an A/B; the odds for students entering in College Algebra decreased by 270\%, 245\% for those entering in Pre-Calculus. This has important implications for physics instruction, because all students in Physics 1 had passed Calculus 1, a pre-requisite for the course; therefore, weak high school mathematics preparation is not completely remediated by matriculating through college mathematics classes.

The performance of the optimal random forest institutional model was better than that of the optimal logistic model producing an accuracy of $72 \%$ and a $\kappa$ of 0.40 representing 


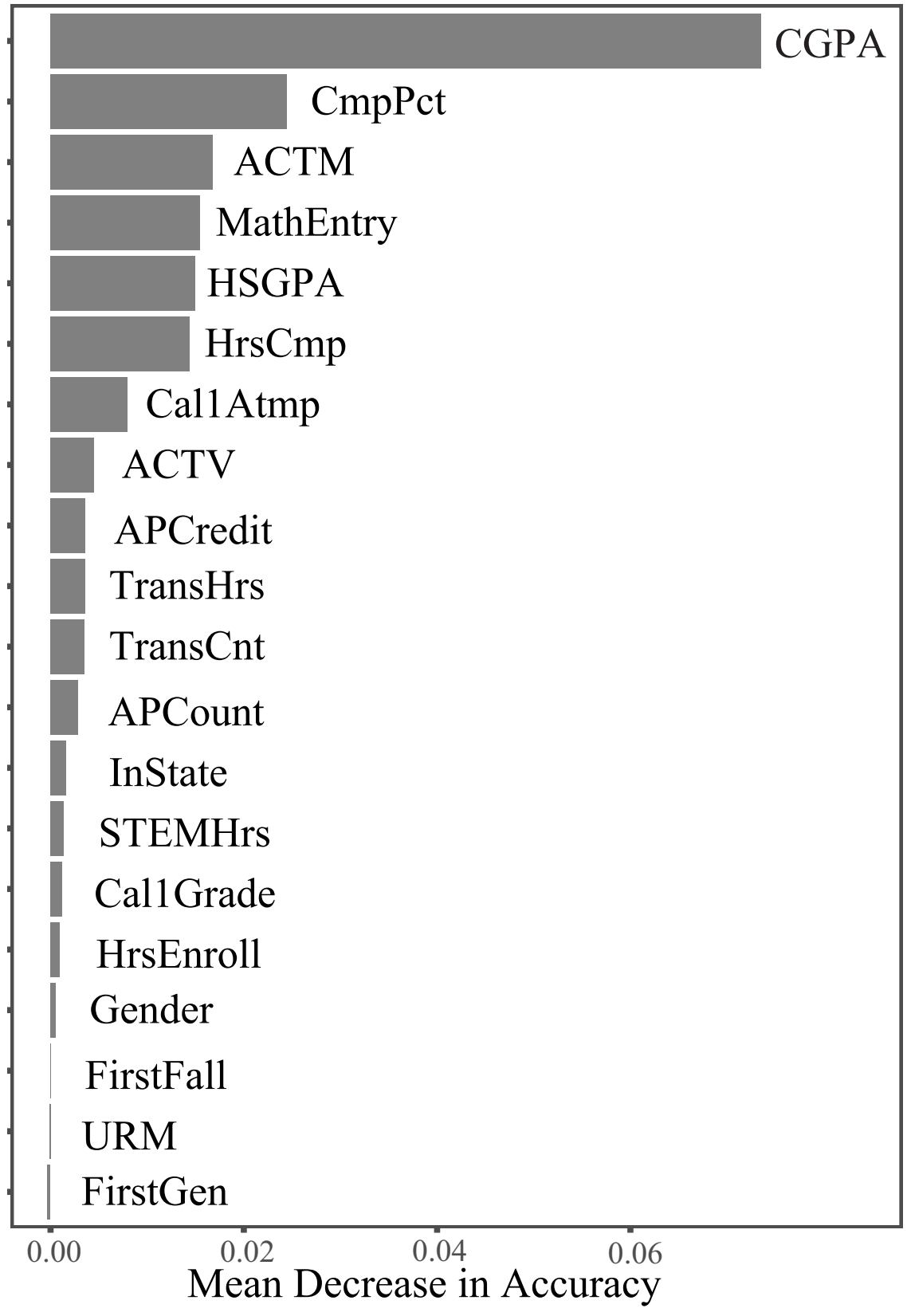

Figure 5.3: Physics 1: Variable importance for institutional variables. High values represent more important variables for the goodness-of-fit of the random forest models. 
moderate agreement of the model with the test data. The model discrimination was somewhat worse than that of the logistic model with an AUC of 0.77 representing adequate model performance. The optimal model selected by the 1-SE rule contained the 4 variables in the logistic model: CGPA, CmpPct, ACTM, and MathEntry, as well as a fifth variable, HrsCmp.

Figure 5.3 plots the mean decrease in accuracy of the institutional random forest model. The mean decrease in accuracy is the average decrease in classification accuracy across all decision trees using the variable if the variable is removed from the tree. Analysis of the importance indices in Fig. 5.3 showed that despite the optimal model requiring only five variables, several other parameters were important when predicting student success in Physics 1. All five of the optimal variables show high or reasonably high importance in Fig. 5.3. The largest drop in importance occurs after CGPA where the next most important parameter was only one third as important to the model fit. Although not included in the optimal model, HSGPA performs very similarly to HrsCmp, MathEntry, and ACTM in variable importance and would likely have been included had CGPA not been included. Demographic factors including first generation status, in-state residence, ethnic or racial minority status, and gender were all minimally important to the model. These results further support the variables selected for the previous logistic model with all 4 of the logistic variables with medium to high importance.

\section{Physics 1 In-Class-Only Models}

The weekly models use in-class and institutional variables on a by-week basis to predict the course grade students will receive with data available in each week. In this section, models using only in-class variables are presented. These models use data that is easily accessible 
Table 5.5: Physics 1: Logistic models in-class variables only.

\begin{tabular}{|c|c|c|c|c|c|}
\hline Variable & $\begin{array}{l}\text { Odds } \\
\text { Ratio }\end{array}$ & $95 \% \mathrm{CI}$ & $\begin{array}{l}\text { Norm } \\
\text { Odds } \\
\text { Ratio }\end{array}$ & Z-score & $p$-value \\
\hline \multicolumn{6}{|c|}{ Week 1} \\
\hline Intercept & 0.01 & {$[0.00,0.02]$} & 0.87 & -6.68 & $<0.001$ \\
\hline FMCEPre & 1.04 & {$[1.02,1.05]$} & 1.88 & 4.60 & $<0.001$ \\
\hline HwkGrade & 1.05 & {$[1.03,1.07]$} & 2.77 & 6.24 & $<0.001$ \\
\hline LecQuiz & 2.34 & {$[1.37,3.98]$} & 2.34 & 3.12 & 0.002 \\
\hline \multicolumn{6}{|c|}{ Week 2} \\
\hline Intercept & 0.00 & {$[0.00,0.00]$} & 1.69 & -8.05 & $<0.001$ \\
\hline FMCEPre & 1.05 & {$[1.03,1.07]$} & 2.33 & 5.19 & $<0.001$ \\
\hline HwkGrade & 1.08 & {$[1.06,1.10]$} & 4.95 & 7.56 & $<0.001$ \\
\hline LecQuiz & 3.58 & {$[1.54,8.34]$} & 1.41 & 2.95 & 0.003 \\
\hline \multicolumn{6}{|c|}{ Week 3} \\
\hline Intercept & 0.00 & {$[0.00,0.00]$} & 0.40 & -9.12 & $<0.001$ \\
\hline FMCEPre & 1.06 & {$[1.04,1.08]$} & 2.91 & 5.64 & $<0.001$ \\
\hline HwkGrade & 1.10 & {$[1.07,1.12]$} & 6.89 & 8.68 & $<0.001$ \\
\hline LecQuiz & 5.47 & {$[1.84,16.24]$} & 1.46 & 3.06 & 0.002 \\
\hline \multicolumn{6}{|c|}{ Week 4} \\
\hline Intercept & 0.00 & {$[0.00,0.00]$} & 0.28 & -9.30 & $<0.001$ \\
\hline FMCEPre & 1.06 & {$[1.04,1.08]$} & 2.84 & 5.55 & $<0.001$ \\
\hline HwkGrade & 1.10 & {$[1.08,1.13]$} & 7.64 & 8.67 & $<0.001$ \\
\hline LecQuiz & 7.90 & {$[2.26,27.58]$} & 1.53 & 3.24 & 0.001 \\
\hline \multicolumn{6}{|c|}{ Week 5} \\
\hline Intercept & 0.00 & {$[0.00,0.00]$} & 0.32 & -10.72 & $<0.001$ \\
\hline FMCEPre & 1.03 & {$[1.01,1.05]$} & 1.74 & 2.75 & 0.006 \\
\hline HwkGrade & 1.10 & {$[1.08,1.13]$} & 7.35 & 8.13 & $<0.001$ \\
\hline LecQuiz & 7.66 & {$[1.76,33.29]$} & 1.51 & 2.72 & 0.007 \\
\hline Test 1 & 1.10 & {$[1.08,1.13]$} & 4.64 & 8.26 & $<0.001$ \\
\hline
\end{tabular}

for most introductory physics instructors. The logistic models from week 1 through week 5 are presented in Table 5.5. Because of the small number of variables available, the random forest analysis will only be discussed in detail for the combined models.

The variables selected by the in-class logistic models were consistent across all five weeks with FMCEPre, HwkGrade, and LecQuiz significant in all models. The normalized odds ratio of the homework grades increased from 2.77 in week 1 to a high of 7.64 in week 4 demonstrating the increasing predictive importance of homework grades as the semester progressed. The odds ratio for the normalized lecture quiz grade was largest in week 1 when the lecture quiz captured whether the student enrolled on time and whether they promptly obtained a clicker as well as first week attendance. In the subsequent weeks, the normalized 
Table 5.6: Physics 1: Logistic models combining in-class and institutional variables.

\begin{tabular}{|c|c|c|c|c|c|}
\hline Variable & $\begin{array}{l}\text { Odds } \\
\text { Ratio }\end{array}$ & $95 \%$ CI & $\begin{array}{l}\text { Norm } \\
\text { Odds } \\
\text { Ratio }\end{array}$ & Z-score & $p$-value \\
\hline \multicolumn{6}{|c|}{ Week 1} \\
\hline Intercept & 0.00 & {$[.00, .00]$} & 5.32 & $\begin{array}{l}-9.34 \\
\end{array}$ & $<0.001$ \\
\hline FMCEPre & 1.04 & {$[1.02,1.06]$} & 1.94 & 3.89 & $<0.001$ \\
\hline HwkGrade & 1.03 & {$[1.02,1.05]$} & 1.96 & 3.95 & $<0.001$ \\
\hline CmpPct & 1.03 & {$[1.02,1.05]$} & 1.66 & 3.68 & $<0.001$ \\
\hline CGPA & 11.91 & {$[6.62,21.44]$} & 3.55 & 8.26 & $<0.001$ \\
\hline $\begin{array}{c}\text { MathEntry: } \\
\text { Algebra }\end{array}$ & 0.23 & {$[0.13,0.43]$} & 0.23 & -4.60 & $<0.001$ \\
\hline $\begin{array}{c}\text { MathEntry: } \\
\text { PreCal }\end{array}$ & 0.29 & {$[0.16,0.53]$} & 0.29 & -4.07 & $<0.001$ \\
\hline \multicolumn{6}{|c|}{ Week 2} \\
\hline Intercept & 0.00 & {$[0.00,0.00]$} & 1.47 & $\begin{array}{l}-9.32 \\
\end{array}$ & $<0.001$ \\
\hline FMCEPre & 1.05 & {$[1.02,1.07]$} & 2.24 & 4.28 & $<0.001$ \\
\hline HwkGrade & 1.05 & {$[1.03,1.08]$} & 2.87 & 4.74 & $<0.001$ \\
\hline LecQuiz & 3.03 & {$[1.13,8.15]$} & 1.35 & 2.20 & 0.028 \\
\hline CmpPct & 1.03 & {$[1.01,1.05]$} & 1.65 & 3.63 & $<0.001$ \\
\hline CGPA & 8.70 & {$[4.73,16.01]$} & 3.02 & 6.95 & $<0.001$ \\
\hline ACTM & 1.02 & {$[1.00,1.04]$} & 1.36 & 2.01 & 0.044 \\
\hline $\begin{array}{c}\text { MathEntry: } \\
\text { Algebra }\end{array}$ & 0.42 & {$[0.19,0.89]$} & 0.42 & -2.25 & 0.024 \\
\hline $\begin{array}{l}\text { MathEntry: } \\
\text { PreCal }\end{array}$ & 0.43 & {$[0.23,0.81]$} & 0.43 & -2.62 & $<.009$ \\
\hline \multicolumn{6}{|c|}{ Week 3} \\
\hline Intercept & 0.00 & {$[0.00,0.00]$} & 1.16 & -9.81 & $<0.001$ \\
\hline FMCEPre & 1.06 & {$[1.03,1.08]$} & 2.72 & 4.89 & $<0.001$ \\
\hline HwkGrade & 1.07 & {$[1.04,1.09]$} & 3.79 & 5.74 & $<0.001$ \\
\hline LecQuiz & 4.35 & {$[1.26,15.00]$} & 1.39 & 2.32 & 0.020 \\
\hline CmpPct & 1.03 & {$[1.01,1.05]$} & 1.63 & 3.57 & $<0.001$ \\
\hline CGPA & 7.65 & {$[4.11,14.21]$} & 2.83 & 6.43 & $<0.001$ \\
\hline $\begin{array}{c}\text { MathEntry: } \\
\text { Algebra }\end{array}$ & 0.33 & {$[0.17,0.63]$} & 0.33 & -3.35 & $<0.001$ \\
\hline $\begin{array}{l}\text { MathEntry: } \\
\text { PreCal }\end{array}$ & 0.43 & {$[0.23,0.81]$} & 0.43 & -2.63 & 0.008 \\
\hline \multicolumn{6}{|c|}{ Week 4} \\
\hline Intercept & 0.00 & {$[0.00,0.00]$} & 0.77 & -9.82 & $<0.001$ \\
\hline FMCEPre & 1.05 & {$[1.03,1.08]$} & 2.59 & 4.72 & $<0.001$ \\
\hline HwkGrade & 1.07 & {$[1.05,1.10]$} & 3.98 & 5.67 & $<0.001$ \\
\hline LecQuiz & 6.49 & {$[1.59,26.50]$} & 1.47 & 2.61 & 0.009 \\
\hline CmpPct & 1.03 & {$[1.01,1.05]$} & 1.63 & 3.56 & $<0.001$ \\
\hline CGPA & 7.09 & {$[3.79,13.25]$} & 2.72 & 6.14 & $<0.001$ \\
\hline $\begin{array}{c}\text { MathEntry: } \\
\text { Algebra }\end{array}$ & 0.33 & {$[0.17,0.64]$} & 0.33 & -3.29 & 0.001 \\
\hline $\begin{array}{l}\text { MathEntry: } \\
\text { PreCal }\end{array}$ & 0.45 & {$[0.24,0.84]$} & 0.45 & -2.52 & 0.012 \\
\hline \multicolumn{6}{|c|}{ Week 5} \\
\hline Intercept & 0.00 & {$[0.00,0.00]$} & 0.40 & -10.69 & $<0.001$ \\
\hline FMCEPre & 1.04 & {$[1.02,1.06]$} & 2.00 & 3.23 & 0.001 \\
\hline HwkGrade & 1.09 & {$[1.06,1.11]$} & 5.54 & 6.69 & $<0.001$ \\
\hline LecQuiz & 7.33 & {$[1.57,34.26]$} & 1.49 & 2.53 & 0.011 \\
\hline CmpPct & 1.03 & {$[1.01,1.05]$} & 1.57 & 3.29 & 0.001 \\
\hline CGPA & 3.72 & {$[1.91,7.24]$} & 1.96 & 3.87 & $<0.001$ \\
\hline Test 1 & 1.09 & {$[1.07,1.12]$} & 4.10 & 7.12 & $<0.001$ \\
\hline
\end{tabular}


coefficient was smaller and fairly constant as the variable transitioned to primarily a measure of attendance.

\section{Physics 1 In-Class and Institutional Models}

Models were also constructed using a combination of in-class and institutional variables. The logistic models are presented in Table 5.6. In weeks 1 to 4, CmpPct, CGPA, and MathEntry were significant institutional variables and FMCEPre, HwkGrade, and LecQuiz were significant in-class variables (LecQuiz was not significant in week 1, with whatever explanatory power it contained in the first week being accounted for by the institutional variables). With the inclusion of Test 1 in week 5, MathEntry was no longer significant. ACTM was only significant in week 2 with a $p$-value of 0.044 . The odds ratio of CmpPct remained relatively stable across all models and MathEntry became fairly constant after week 1. The normalized CGPA odds ratio decreased from a high of 3.55 in week 1 to a low of 1.96 in week 5 when Test 1 grade became available.

Random forests provided additional insight by estimating variable importance which characterizes the value of each parameter without sensitivity to multicollinearity. These models combine the optimal institutional model with the optimal in-class model without further pruning. Figure 5.4 shows that CGPA was the most important variable in weeks 1 to 4 by a factor of at least 2 when compared to every other variable except homework grade. Test 1 became the most important variable in week 5 . The random forest models routinely performed more weakly than the logistic models by a small margin.

In all weeks, the in-class variables FMCEPre and LecQuiz were of little importance to the accuracy of the random forest models. This does not agree with the parameter 
Week 1

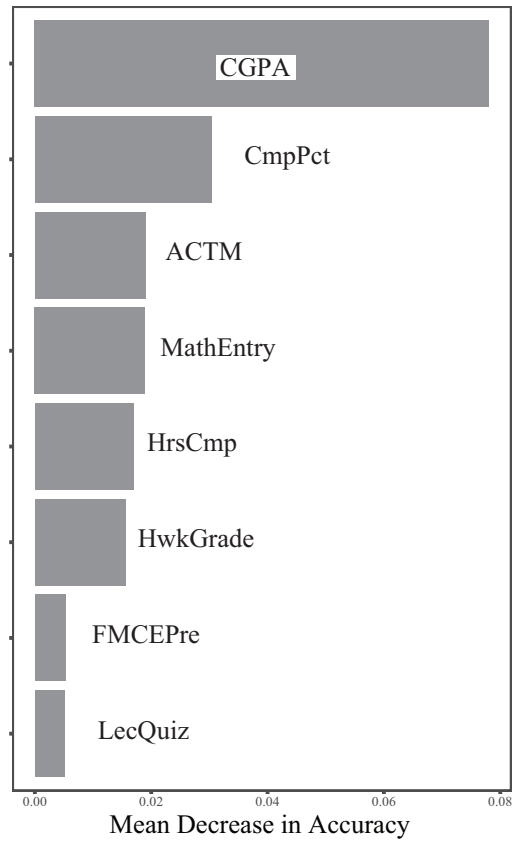

Week 4

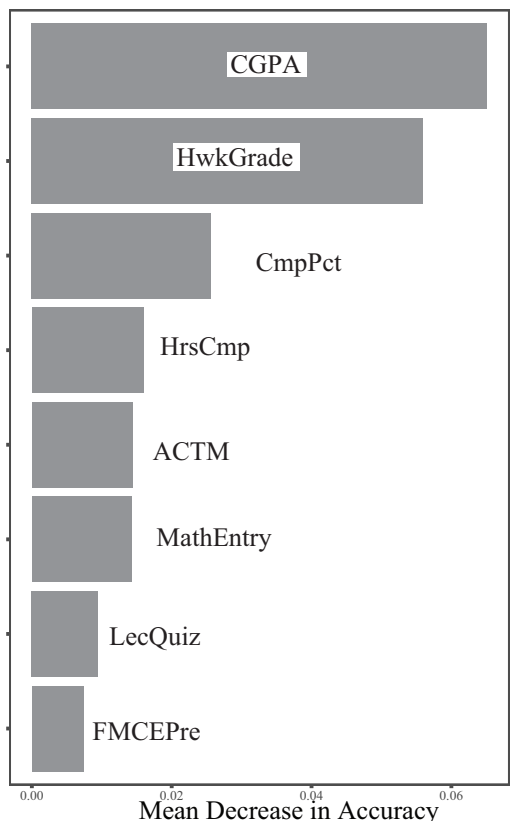

Mean Decrease in Accuracy
Week 2

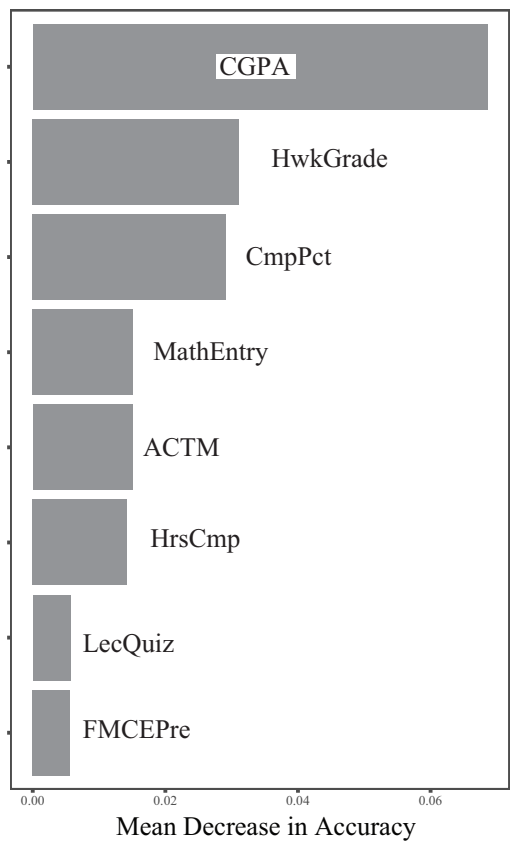

Mean Decrease in Accuracy
Week 3

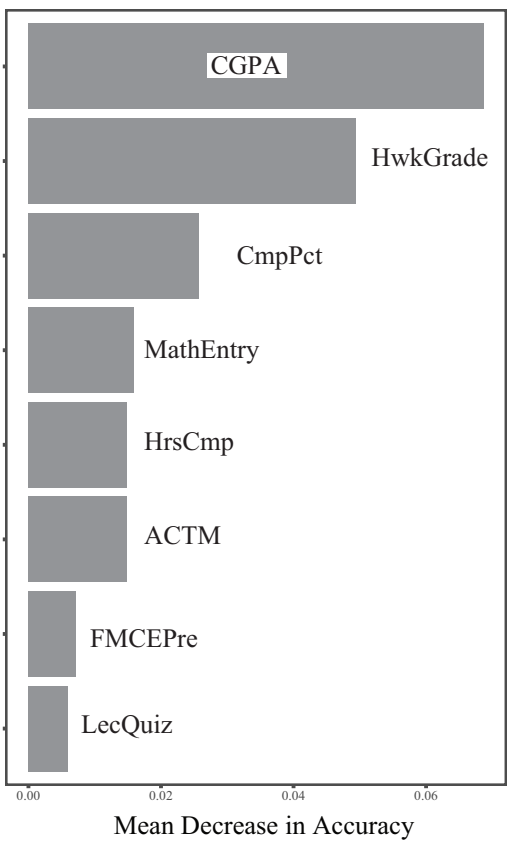

Week 5

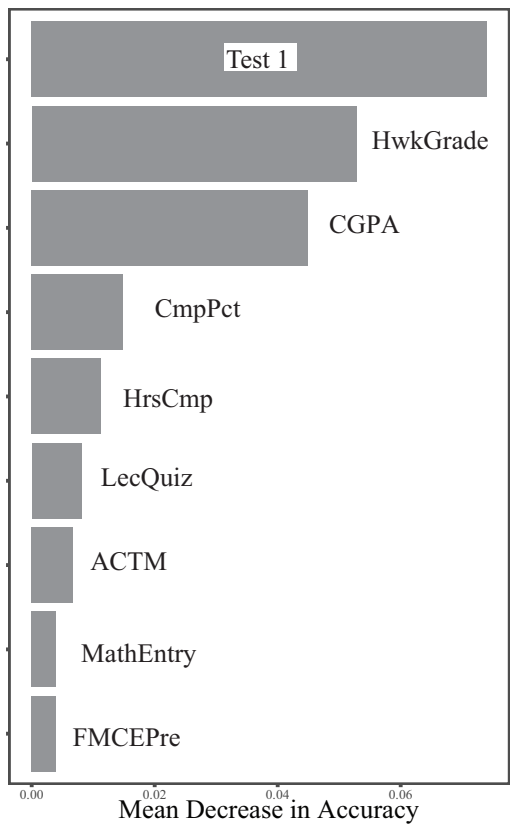

Figure 5.4: Physics 1: Weekly variable importance of the combined in-class/institutional models. 


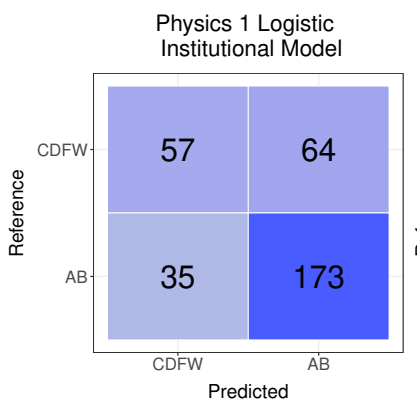

(a)

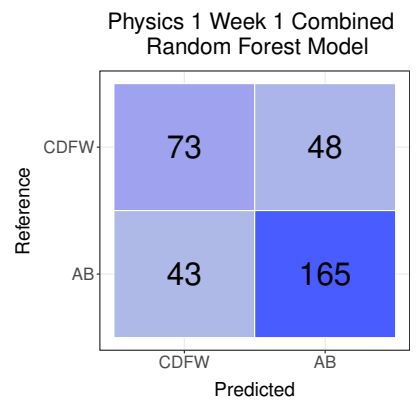

(d)

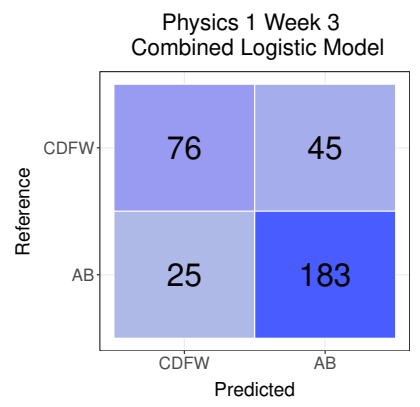

(g)

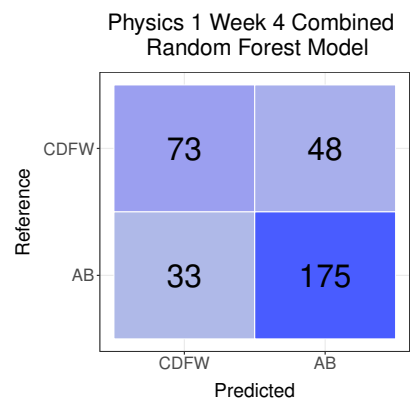

(j)

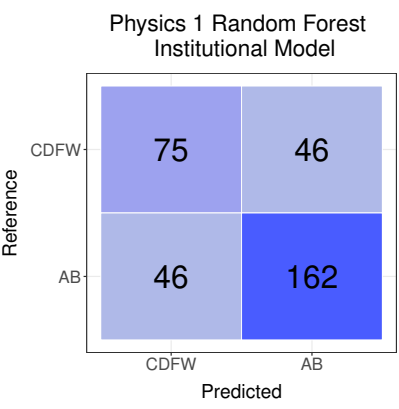

(b)

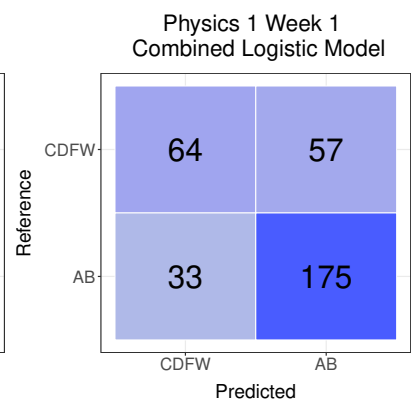

(c)

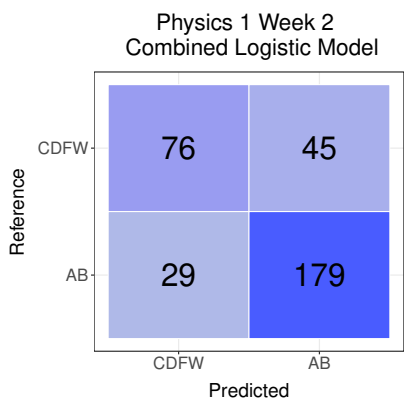

(e)

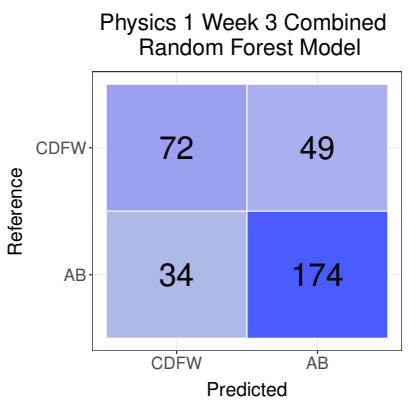

(h)

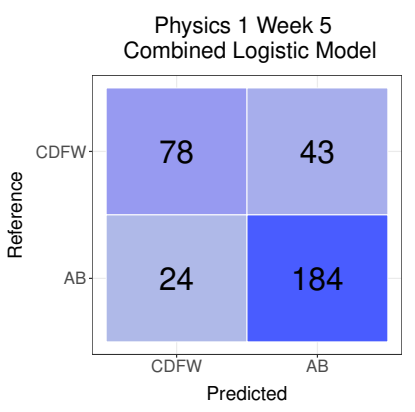

(k)

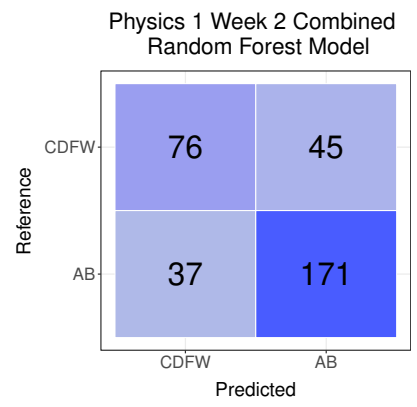

(f)

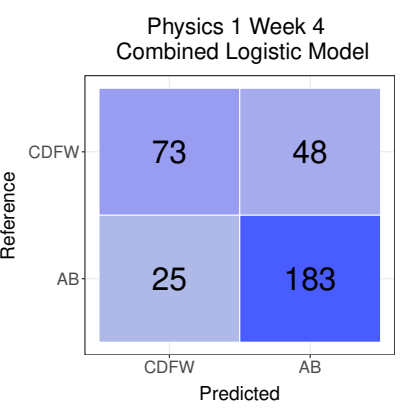

(i)

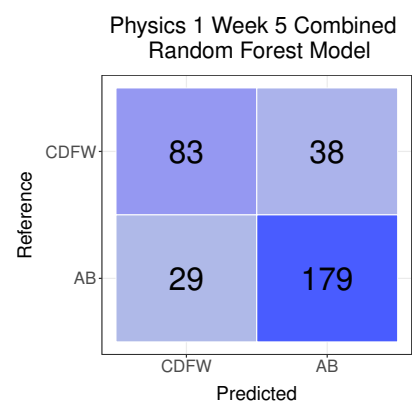

(1)

Figure 5.5: Physics 1 Optimal Model Confusion Matrices 
estimates of the logistic models in Table 5.6 where FMCEPre often had a normalized odds ratio commensurate with HwkGrade. This suggests the collinearity expected between the grades of different assignments in the same class may be influencing the parameter estimates of grades in the logistic models. This shows the efficacy of using logistic and random forest models in parallel.

Figure 5.5 shows the confusion matricies of the optimal by-week models in Physics 1. The logistic models tend to have a bias toward false positives over false negatives. This will identify fewer students as requiring early intervention and thus tax instructional resources less than a model balanced between false negative and false positive at the expense of misidentifying a large portion of students as more likely to be successful than their are. Depending upon instructional resources, this balance can be tuned to optimize how limited resources are used. The random forest models, though less accurate, are more balanced in terms of misclassification as either $\mathrm{AB}$ or $\mathrm{CDFW}$.

\subsubsection{Physics 2 Model Performance and Variable Importance}

Physics 2 is usually taken the semester after Physics 1 and has Physics 1 as its prerequisite. As such, the Physics 2 models contain additional important variables not available to the Physics 1 models: the grade in Physics 1 (P1Grade) and whether Physics 1 was taken more than once (P1Atmp). Physics 2 also maintained more detailed weekly records allowing the use of lab grades and lab quiz grades. 
Table 5.7: Physics 2: Logistic and Random Forest Model Performance. The 95\% CI for AUC is shown in brackets

\begin{tabular}{c|c|c|c|c|c||c|c|c}
\hline \multicolumn{1}{c|}{} & \multicolumn{4}{|c||}{ Logistic Regression } & \multicolumn{4}{c}{ Random Forests } \\
\hline Models & Variables & Accuracy & $\kappa$ & AUC & $R^{2}$ & Accuracy & $\kappa$ & AUC \\
\hline Baseline & None & 0.68 & 0.00 & 0.50 & & 0.68 & 0.00 & 0.50 \\
\hline \multirow{2}{*}{ Institutional } & All Variables & 0.81 & 0.57 & $0.89[0.86,0.93]$ & 0.34 & 0.80 & 0.55 & $0.88[0.84,0.92]$ \\
& Optimal & 0.80 & 0.55 & $0.89[0.85,0.93]$ & 0.31 & 0.81 & 0.58 & $0.86[0.82,0.90]$ \\
\hline \multirow{2}{*}{ Week 1 } & In-Class & 0.73 & 0.33 & $0.75[0.69,0.81]$ & 0.12 & 0.74 & 0.36 & $0.74[0.68,0.80]$ \\
& In-Class/Institutional & 0.81 & 0.57 & $0.90[0.87,0.94]$ & 0.35 & 0.81 & 0.57 & $0.88[0.84,0.92]$ \\
\hline \multirow{2}{*}{ Week 2 } & In-Class & 0.75 & 0.40 & $0.78[0.73,0.84]$ & 0.21 & 0.75 & 0.42 & $0.75[0.70,0.81]$ \\
& In-Class/Institutional & 0.81 & 0.57 & $0.91[0.87,0.94]$ & 0.38 & 0.82 & 0.61 & $0.88[0.84,0.92]$ \\
\hline \multirow{2}{*}{ Week 3 } & In-Class & 0.78 & 0.49 & $0.85[0.80,0.89]$ & 0.30 & 0.79 & 0.52 & $0.87[0.82,0.91]$ \\
& In-Class/Institutional & 0.82 & 0.59 & $0.91[0.88,0.94]$ & 0.42 & 0.84 & 0.62 & $0.91[0.87,0.94]$ \\
\hline \multirow{2}{*}{ Week 4 } & In-Class & 0.78 & 0.48 & $0.85[0.81,0.90]$ & 0.31 & 0.75 & 0.43 & $0.83[0.78,0.88]$ \\
& In-Class/Institutional & 0.84 & 0.62 & $0.91[0.88,0.95]$ & 0.44 & 0.81 & 0.57 & $0.89[0.85,0.92]$ \\
\hline \multirow{2}{*}{ Week 5 } & In-Class & 0.85 & 0.65 & $0.94[0.91,0.97]$ & 0.56 & 0.85 & 0.66 & $0.93[0.90,0.96]$ \\
& In-Class/Institutional & 0.85 & 0.67 & $0.95[0.93,0.97]$ & 0.60 & 0.86 & 0.68 & $0.95[0.92,0.97]$ \\
\hline
\end{tabular}

\section{Institutional Model}

The accuracy, $\kappa$, and AUC of each Physics 2 model is presented in the Table 5.7. The optimal logistic model using only institutional variables produced an accuracy of $80 \%$, an improvement of $12 \%$ over the baseline model and $10 \%$ over the performance of the same model in Physics 1. This model also had $\kappa$ of 0.55 and AUC of 0.89 , values that were not obtained until the week 5 models in Physics 1.

The optimal logistic model of the institutional data for Physics 2 was strongly related to performance in Physics 1 as shown in Table 5.8. The normalized odds ratios for receiving an A/B in Physics 2 improved by $169 \%$ if the student received an A/B in Physics 1. CGPA remained an influential variable with a one standard deviation improvement in CGPA improving the odds of receiving an A/B in Physics 2 by 243\%. Taking Physics 1 more than a single time decreased a student's odds of receiving an A/B by $426 \%$. Physics 1 is the direct prerequisite for Physics 2 and, therefore, this result was not surprising; however, Calculus 1 is a direct prerequisite for Physics 1, but was not significant in any of the Physics 1 models. Cal1Grade was significant in the Physics 2 random forest models. 
Table 5.8: Physics 2: Optimal institutional logistic model.

\begin{tabular}{c|c|c|c|c|c}
\hline Variable & $\begin{array}{c}\text { Odds } \\
\text { Ratio }\end{array}$ & $95 \%$ CI & $\begin{array}{c}\text { Norm } \\
\text { Odds } \\
\text { Ratio }\end{array}$ & Z-score & $p$ \\
\hline Intercept & 0.00 & {$[0.00,0.01]$} & 1.76 & -7.53 & $<0.001$ \\
P1Grade & 2.69 & {$[1.56,4.63]$} & 2.69 & 3.57 & $<0.001$ \\
P1Atmp & 0.19 & {$[0.07,0.54]$} & 0.19 & -3.12 & 0.002 \\
CGPA & 11.66 & {$[6.01,22.59]$} & 3.43 & 7.27 & $<0.001$ \\
\hline
\end{tabular}

The institutional random forest models for Physics 2 also performed very well with the optimal institutional model achieving an accuracy of $81 \%, \kappa$ of 0.58 , and AUC of 0.86. This high degree of predictive power was achieved using CGPA, P1Grade, Cal1Grade, HrsCmp, and CmpPct. The variables CGPA, HrsCmp, and CmpPct were also included in the corresponding random forest model in Physics 1. The variable importance indices in Fig. 5.6 of the full model show similar results to Physics 1 with CGPA the highest importance variable with a mean decrease in accuracy of more than twice that of the next most important variable. The demographic variables were once again unimportant for predicting grade. Only a small subset of institutional variables were necessary to build highly performing models of student course success in the random forest model.

\section{Weekly Models}

The performance of the optimal logistic model of the institutional data was not matched by the in-class-only model until week 5 and Test 1, much later than the Physics 1 logistic models. However, all of the logistic models were highly accurate with all combined institutional/inclass models having accuracies in excess of $80 \%$, Cohen's kappas in the range of good agreement, and excellent discrimination characteristics as measured by AUC. In each case, the Physics 2 models performed better than similar models in Physics 1. This may have 


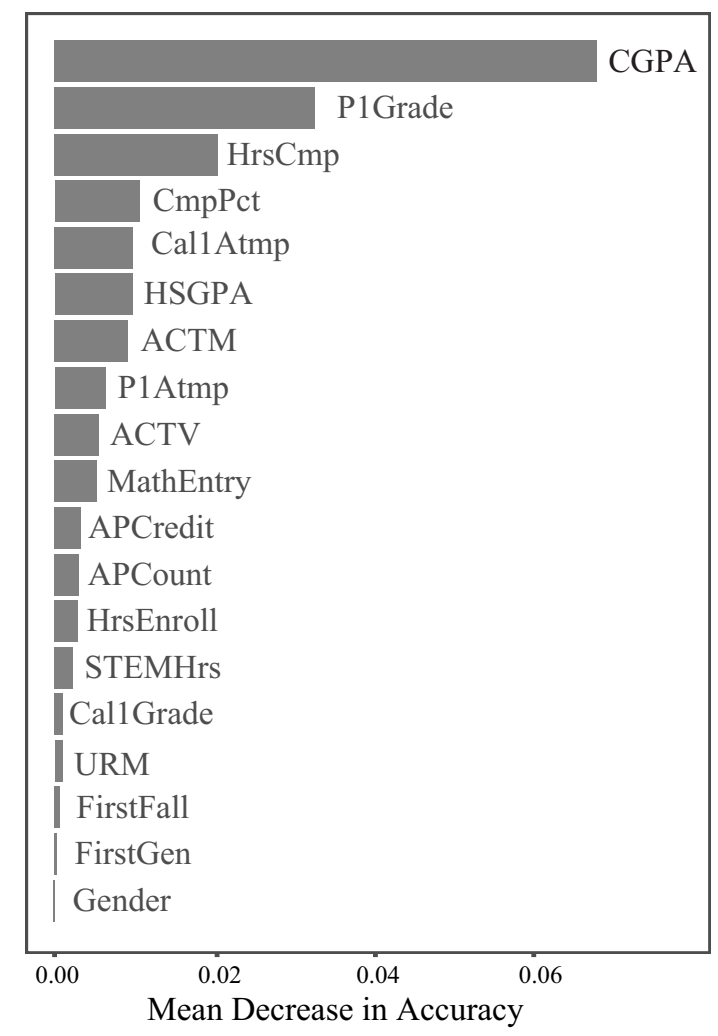

Figure 5.6: Physics 2 variable importance for the institutional variables.

resulted from the additional in-class variables available, the availability of P1Grade as a variable, the increased difficulty and unfamiliarity of the material, or from hand-graded homework providing a more accurate measure of students' understanding.

The in-class logistic models were remarkably consistent from week 1 to week 5 as shown int Table 5.9, with only LabQuiz and HwkGrade significant in the first 4 weeks. The week 5 model retained both parameters along with Test 1 grade. Unlike the behavior of LecQuiz in Physics 1, there was no large difference in the LabQuiz normalized odds ratio between week 1 and week 2. The normalized odds ratio for HwkGrade increased from 2.13 in week 1 to 4.40 in week 5 showing the increasing importance of homework grades for predicting student success in this course.

The combined institutional/in-class models, shown in Table 5.10 also performed similarly 
Table 5.9: Physics 2: Logistic models in-class variables only.

\begin{tabular}{c|c|c|c|c|c}
\hline Variable & $\begin{array}{c}\text { Odds } \\
\text { Ratio }\end{array}$ & $95 \%$ CI & $\begin{array}{c}\text { Norm } \\
\text { Odds } \\
\text { Ratio }\end{array}$ & Z-score & $p$-value \\
\hline \multicolumn{5}{c}{ Week 1} \\
\hline Intercept & 0.12 & {$[0.05,0.28]$} & 2.47 & -4.71 & $<0.001$ \\
LabQuiz & 1.14 & {$[1.05,1.25]$} & 1.39 & 3.11 & 0.002 \\
HwkGrade & 1.59 & {$[1.40,1.81]$} & 2.13 & 7.14 & $<0.001$ \\
\hline \multicolumn{5}{c}{ Week 2} \\
\hline Intercept & 0.02 & {$[0.00,0.08]$} & 2.72 & -4.89 & $<0.001$ \\
LabQuiz & 1.24 & {$[1.04,1.47]$} & 1.32 & 2.38 & 0.017 \\
HwkGrade & 2.36 & {$[1.97,2.82]$} & 3.24 & 9.32 & $<0.001$ \\
\hline \multicolumn{5}{c}{ Week 3 } \\
\hline Intercept & 0.00 & {$[0.00,0.01]$} & 3.09 & -7.33 & $<0.001$ \\
LabQuiz & 1.47 & {$[1.23,1.75]$} & 1.70 & 4.29 & $<0.001$ \\
HwkGrade & 3.04 & {$[2.42,3.80]$} & 4.13 & 9.68 & $<0.001$ \\
\hline \multicolumn{5}{c}{ Week 4 } \\
\hline Intercept & 0.00 & {$[0.00,0.01]$} & 3.17 & -7.89 & $<0.001$ \\
LabQuiz & 1.60 & {$[1.33,1.92]$} & 1.92 & 5.03 & $<0.001$ \\
HwkGrade & 2.96 & {$[2.36,3.71]$} & 4.00 & 9.39 & $<0.001$ \\
\hline \multicolumn{6}{c}{ Week 5 } \\
\hline Intercept & 0.00 & {$[0.00,0.00]$} & 6.21 & -9.22 & $<0.001$ \\
LabQuiz & 1.33 & {$[1.04,1.71]$} & 1.47 & 2.25 & 0.025 \\
HwkGrade & 3.26 & {$[2.38,4.47]$} & 4.40 & 7.35 & $<0.001$ \\
Test 1 & 1.06 & {$[1.05,1.08]$} & 8.53 & 8.95 & $<0.001$ \\
\hline
\end{tabular}

to those of Physics 1 with all variables except CGPA and LabQuiz consistently significant over the five weeks. As the course accumulated grade data, the normalized odds ratio of CGPA dropped from 2.89 in week 1 to 2.17 in week 4; in week 5, when the Test 1 grade was available, CGPA was no longer significant. LabQuiz was only significant in weeks 3 and 4.

The random forest results in Table 5.7 show that the random forest models performed similarly to the logistic models. The in-class model was not an improvement over the institutional model until week 5 .

Variable importance in Physics 2 was similar the variable importance in Physics 1 with CGPA the most important variable and homework grade increasing in importance throughout the five week period to match CGPA in week 5. Unlike Physics 1, when Test 
Week 1

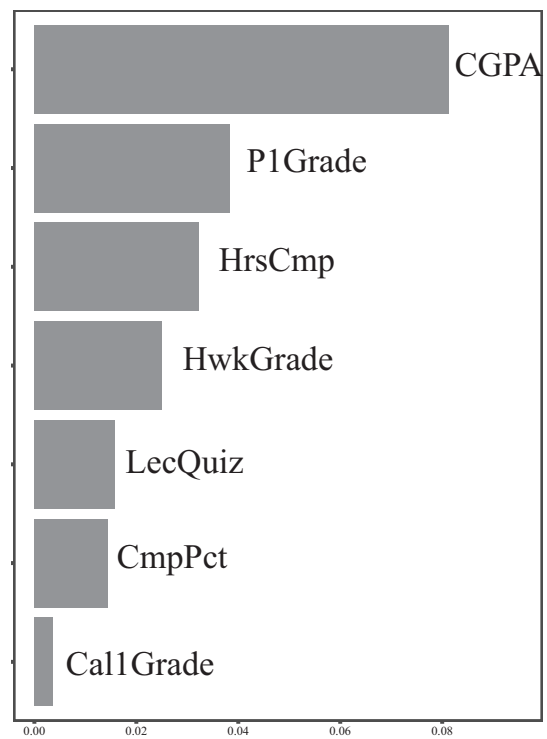

Mean Decrease in Accuracy
Week 2

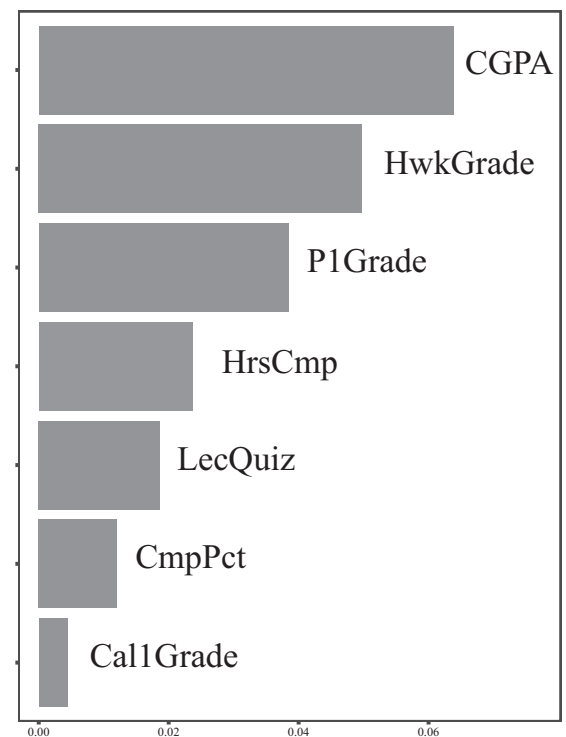

Mean Decrease in Accuracy
Week 3

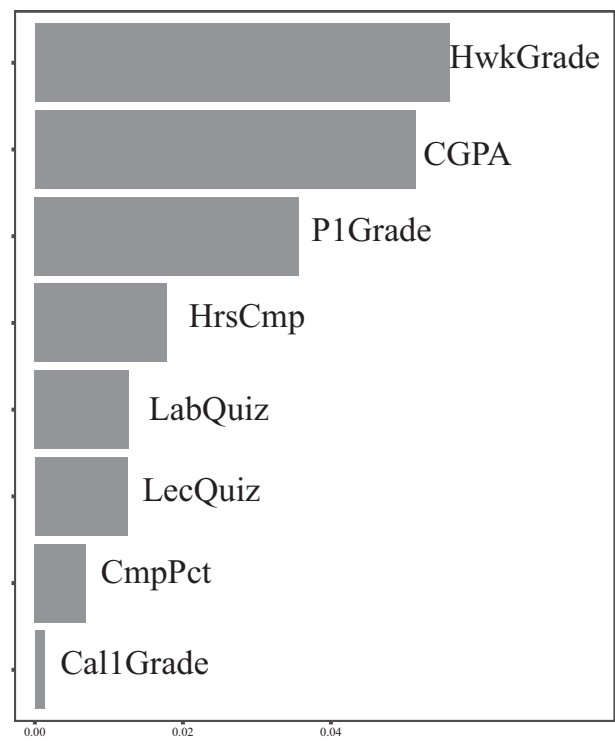

Mean Decrease in Accuracy

Week 4

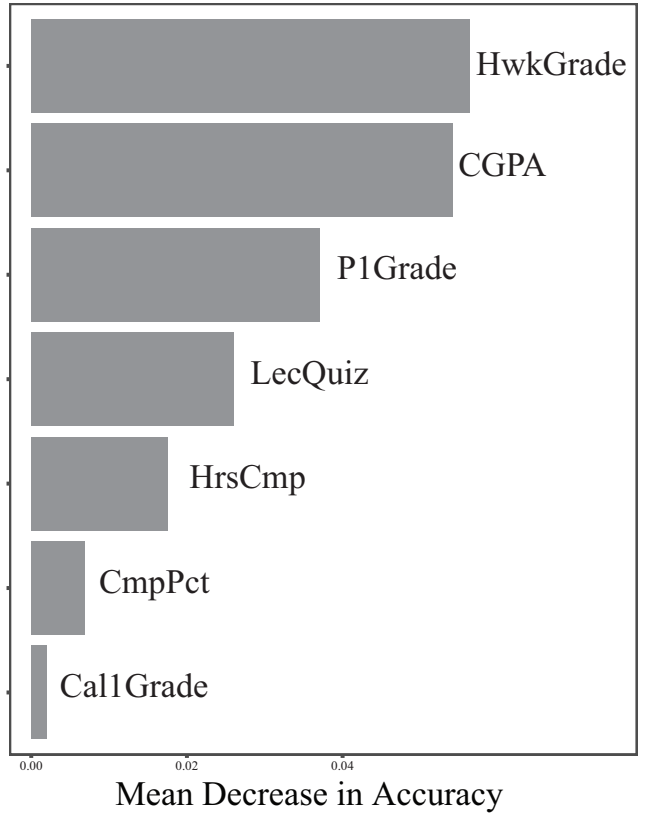

Week 5

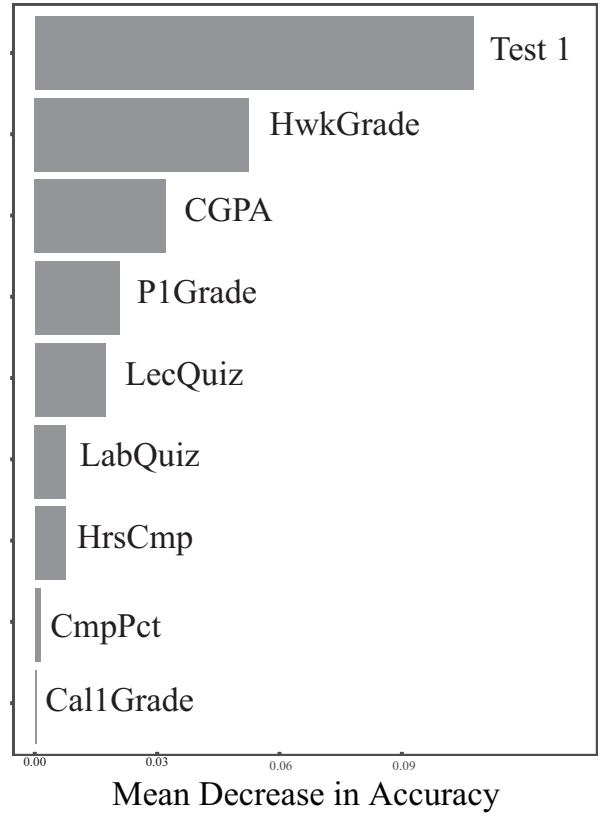

Figure 5.7: Physics 2 variable importance for week 1 to 5 . High values represent move important variables to the goodness of fit of the random forest models. 
Table 5.10: Physics 2: Logistic models in-class and institutional variables only.

\begin{tabular}{c|c|c|c|c|c}
\hline Variable & $\begin{array}{c}\text { Odds } \\
\text { Ratio }\end{array}$ & $95 \%$ CI & $\begin{array}{c}\text { Norm } \\
\text { Odds } \\
\text { Ratio }\end{array}$ & Z-score & $p$-value \\
\hline \multicolumn{5}{c}{ Week 1 } \\
\hline Intercept & 0.00 & {$[0.00,0.03]$} & 9.29 & -4.87 & $<0.001$ \\
HwkGrade & 1.40 & {$[1.21,1.62]$} & 1.72 & 4.45 & $<0.001$ \\
CGPA & 8.29 & {$[4.15,16.58]$} & 2.89 & 5.98 & $<0.001$ \\
P1Grade & 3.15 & {$[1.78,5.55]$} & 3.15 & 3.96 & $<0.001$ \\
P1Atmp & 0.18 & {$[0.06,0.50]$} & 0.18 & -3.26 & 0.001 \\
\hline \multicolumn{5}{c}{ Week 2 } \\
\hline Intercept & 0.00 & {$[0.00,0.02]$} & 8.94 & -4.90 & $<0.001$ \\
HwkGrade & 1.86 & {$[1.52,2.28]$} & 2.34 & 6.05 & $<0.001$ \\
CGPA & 6.51 & {$[3.17,13.36]$} & 3.13 & 3.86 & $<0.001$ \\
P1Grade & 3.13 & {$[1.75,5.60]$} & 3.13 & 3.86 & $<0.001$ \\
P1Atmp & 0.19 & {$[0.07,0.56]$} & 0.19 & -3.04 & 0.002 \\
\hline \multicolumn{5}{c}{ Week 3 } \\
\hline Intercept & 0.00 & {$[0.00,0.01]$} & 10.82 & -5.29 & $<0.001$ \\
HwkGrade & 2.36 & {$[1.84,3.02]$} & 2.99 & 6.78 & $<0.001$ \\
LabQuiz & 1.34 & {$[1.10,1.65]$} & 1.50 & 2.86 & 0.004 \\
CGPA & 4.64 & {$[2.19,9.83]$} & 2.16 & 4.01 & $<0.001$ \\
P1Grade & 3.03 & {$[1.64,5.59]$} & 3.03 & 3.54 & $<0.001$ \\
P1Atmp & 0.18 & {$[0.06,0.54]$} & 0.18 & -3.05 & 0.002 \\
\hline \multicolumn{6}{c}{ Week 4 } \\
\hline Intercept & 0.00 & {$[0.00,0.00]$} & 12.22 & -5.80 & $<0.001$ \\
LabQuiz & 1.54 & {$[1.25,1.89]$} & 1.81 & 4.02 & $<0.001$ \\
HwkGrade & 2.32 & {$[1.81,2.97]$} & 2.93 & 6.61 & $<0.001$ \\
CGPA & 4.66 & {$[2.18,9.94]$} & 2.17 & 3.98 & $<0.001$ \\
P1Grade & 3.03 & {$[1.62,5.65]$} & 3.03 & 3.48 & $<0.001$ \\
P1Atmp & 0.17 & {$[0.06,0.50]$} & 0.17 & -2.17 & 0.002 \\
\hline Intercept & 0.00 & {$[0.00,0.00]$} & 14.39 & -7.39 & $<0.001$ \\
HwkGrade & 3.30 & {$[2.36,4.59]$} & 4.46 & 7.03 & $<0.001$ \\
P1Grade & 4.86 & {$[2.50,9.44]$} & 4.86 & 4.67 & $<0.001$ \\
P1Atmp & 0.17 & {$[0.04,0.67]$} & 0.17 & -2.52 & 0.012 \\
Test 1 & 1.06 & {$[1.05,1.08]$} & 8.18 & 8.53 & $<0.001$ \\
\hline
\end{tabular}

1 was included in week 5, it was nearly twice as important as either CGPA or HwkGrade.

Unlike with Physics 1, the confusion matricies in Fig. 5.8 show the both the logistic models and the random forest models are biased toward false positive results. 


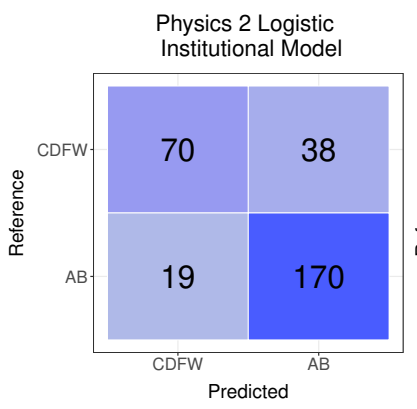

(a)

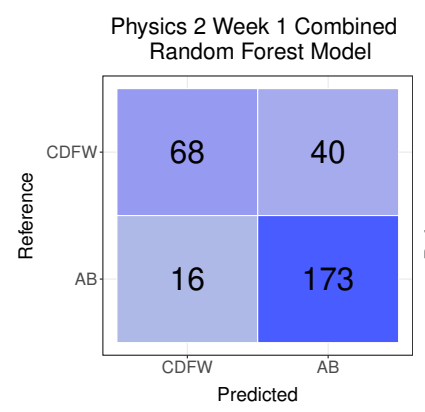

(d)

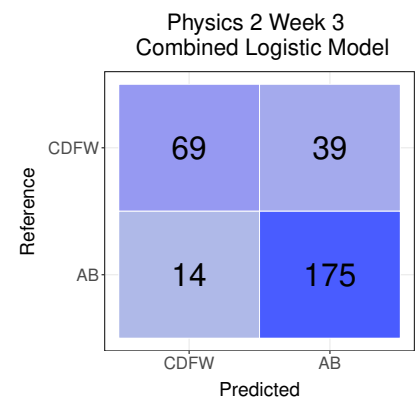

(g)

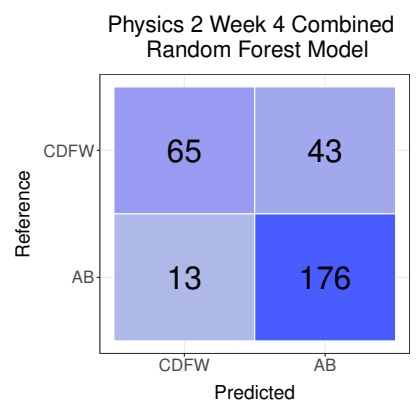

(j)

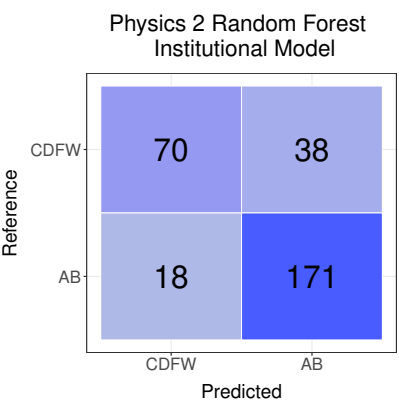

(b)

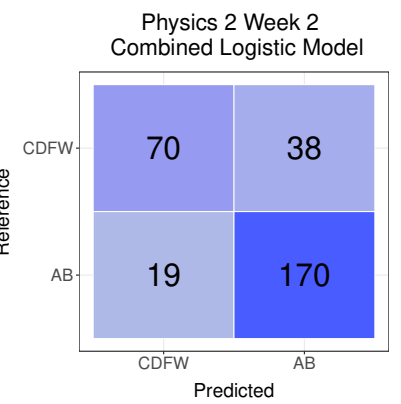

(e)

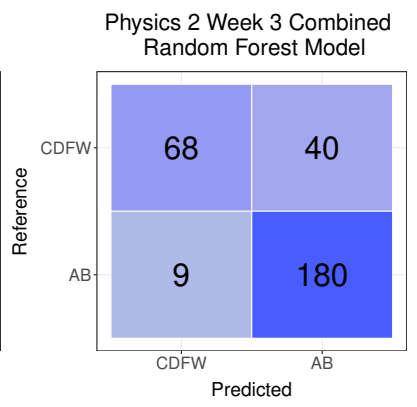

(h)

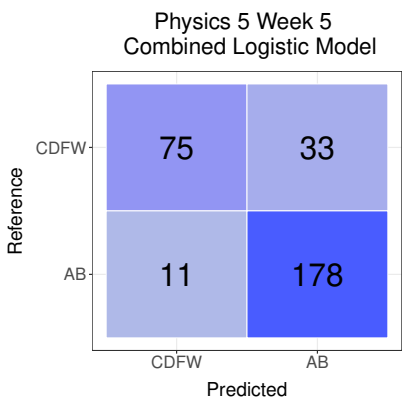

(k)

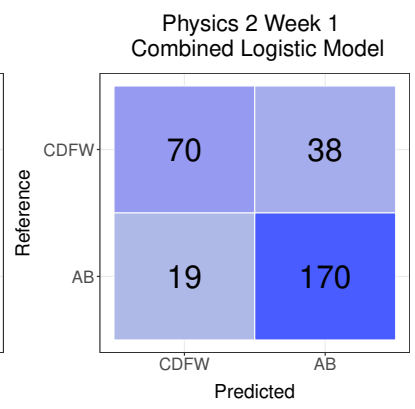

(c)

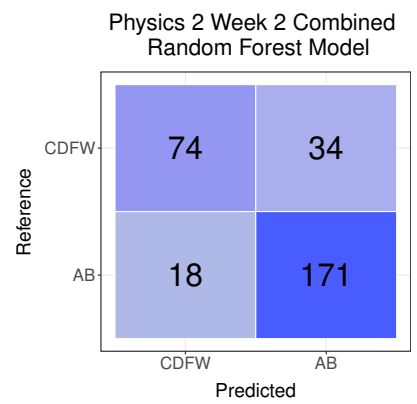

(f)

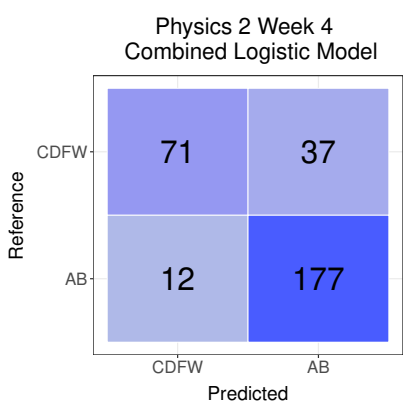

(i)

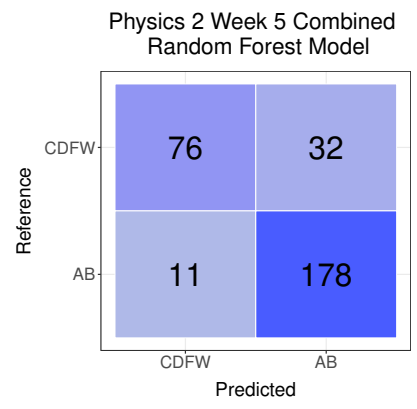

(1)

Figure 5.8: Physics 2 Optimal Model Confusion Matrices 


\subsubsection{LIME Results}

Once a classification model is constructed, the variable importance and the odds ratios can help instructors and researchers understand the factors that are important for predicting students' success. The classification model can then be used to identify students at risk of receiving a low grade. The LIME algorithm allows further understanding of the classification model, showing the factors that were important to making the prediction for individual students.

Figure 5.9 shows the LIME analysis of the progression of the optimal logistic model for a single Physics 1 student in weeks 1, 3, and 5. The student ultimately earned a "B" in the class. In the first week of the class, the model predicted that the student would receive an "A" or "B" with $61 \%$ probability. Poor performance on the first in-class homework assignment, a relatively weak ACTM score, and a weak CGPA were the largest contributors to lowering the probability, shown in Fig 5.9(a). By week 3, the probability of earning an "A" or "B" was $62 \%$. The student's low homework grade was the strongest factor lowering this probability. A strong record of previous course completion, strong lecture quiz scores, and a fair (for the class) score on the FMCE pretest supported the prediction of earning an A/B. By week 5, the probability increased dramatically due to a very strong performance on the first examination. Despite a number of variables indicating that this student would not do well in the course prior to week 5 , the model accurately predicted that the student would receive a " $\mathrm{B}$ " or better in the first week of class. 
(a) Week 1: Probability of Earning A or B of $61 \%$.

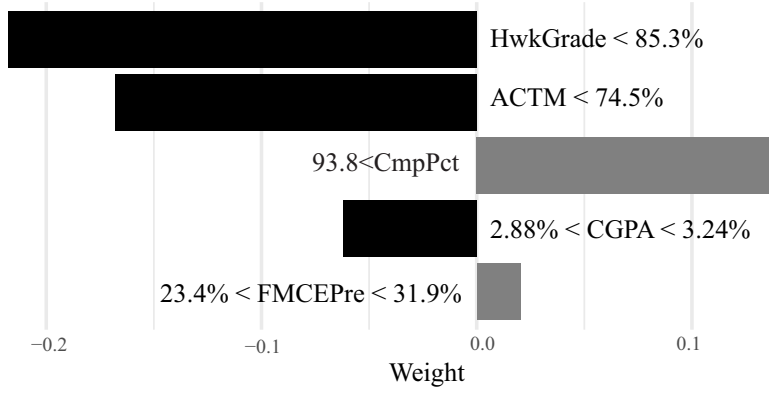

(c) Week 5: Probability of Earning A or B of $91 \%$.

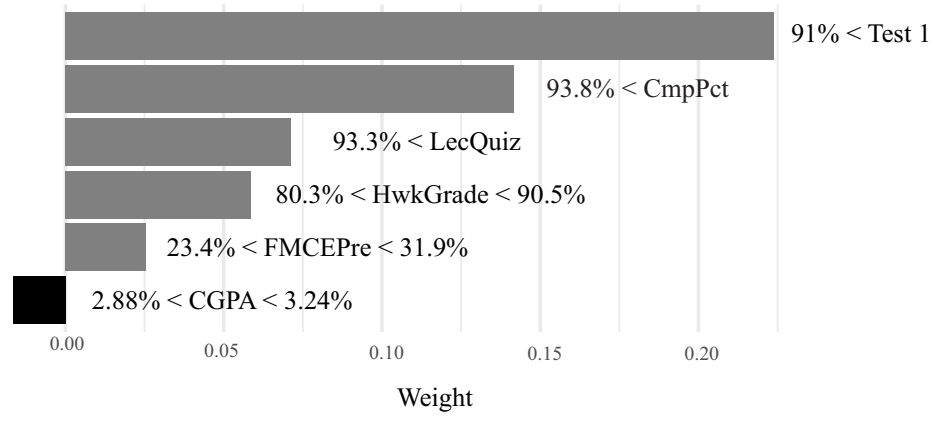

(b) Week 3: Probability of Earning A or B of $62 \%$.
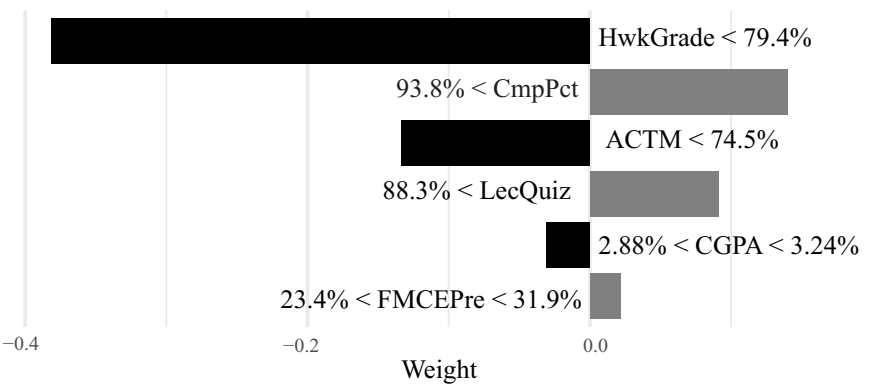

Figure 5.9: Physics 1 LIME results for one student for weeks 1 , week 3 , and week 5 . The student received a "B" in the class.

\subsection{Discussion}

This study investigated two research questions; they will be discussed in the order proposed.

RQ1: How well can introductory physics course grades be predicted early in the semester? Highly accurate models of student success were constructed in both Physics 1 and Physics 2 using institutional variables, in-class variables, and a combination of in-class and institutional variables. In both classes, the models with only institutional variables performed well with strong fit statistics. These models outperformed similar models reported by Huang and Fang [146] that had a maximum classification prediction accuracy of $67 \%$ compared to the $70 \%$ to $80 \%$ for our logistic models. The optimal logistic institutional-only models outperformed, as measured by $\kappa$, models using only in-class variables early in the class. In 
Physics 1, three weeks of in-class data were required before in-class-only models matched the performance of the institutional-only models. In Physics 2, in-class-only models did not match the performance of institutional-only models until week 5 when the score on the first in-semester examination became available. In Physics 1, models combining institutional and in-class variables had superior performance, measured by $\kappa$, in week 2 to in-class-only models in week 4; thus allowing the accurate identification of at-risk students much earlier in the semester. In Physics 2, the week 1 combined institutional/in-class model had superior properties to the week 4 in-class-only model, allowing very early identification of at-risk students. This early identification may allow interventions to be directed toward students in time to positively affect their first test grade.

The in-class models presented in this study performed similarly to those developed by Macfadyen and Dawson [148], but were around $10 \%$ to $15 \%$ less accurate than the inclass models developed by Marbouti et al. [147]. There are many potential reasons for the lower accuracy of our models: the current study used grades less than a "B" as a classification criteria while Marbouti et al. classified grades less than a "C." The course studied by Marbouti et al. was an introductory engineering course which had students with a fairly homogeneous preparation and may have weighted participation more strongly than mastery of content. Physics 1 and 2, as well as the introductory biology course studied by Macfadyen and Dawson [148], are courses taken by a variety of majors at different points in their academic careers.

RQ2: What variables are most important for the accurate prediction of physics course grades early in the semester? In both courses, the institutional models were constructed using only a small subset of available parameters: CGPA, CmpPct, ACTM, and MathEntry 
in Physics 1 and CGPA, P1Grade, and P1Atmp in Physics 2. As such, only a limited amount of additional information would need to be provided to an instructor to greatly increase the accuracy of the identification of at-risk students. Additional institutional variables are important predictors of student success; however, they were unnecessary for the identification of at-risk students in the introductory physics courses in this study.

The differences in variable selection between the two courses was most likely the result of the availability of Physics 1 course information for the Physics 2 models. Physics 1 performance serves as a strong indicator of student performance in a Physics 2. The high importance of CGPA is consistent with other research into grade prediction [146, 152]. As the direct prerequisite for Physics 2, Physics 1 grade was expected to be the strongest predictor; however, its variable importance was less than CGPA showing that overall academic performance at the college level was more important than prior physics performance. The grade in the primary prerequisite course for the Physics 1, Calculus 1, was not as important as was expected from prior research $[146,152]$. The grade in Calculus 1 was not important to the Physics 1 models and was only significant in the Physics 2 models. In Physics 1, the student's math entry point was an important variable in both the institutional only and the combined models. All students in Physics 1 had completed Calculus 1; therefore, completing a college mathematics class did not completely remove the effects of weak high school mathematics preparation. This was consistent with previous work showing prior mathematics performance was predictive of current course performance $[146,150]$.

For the in-class models for both courses, HwkGrade was the most important variable until Test 1 grade became available. In the courses studied, the student's homework grade 
was the variable most directly related to mastery of the topics tested by the in-semester examinations. The importance of this variable increased as the class progressed. This may have been a result of the increasing weekly difficulty of assignments early in a physics class providing a better measure of student capability. It could also result from HwkGrade measuring whether the student self-regulates to improve homework scores early in the class. HwkGrade performs much better in Physics 2 than in Physics 1. In Physics 1 the homework is distributed using a web-based system and is susceptible to large scale cheating, with many of the answers to questions available online. In contrast the Physics 2 course uses instructor written homework which students must hand-solve. These assignments are graded by TAs and therefore the work is graded in addition to the final answer. The increased utility of HwkGrade in Physics 2 provides strong evidence for the usefulness of instructor written homework over using those provided by an external service.

The conceptual pretest was of lower importance than homework grade in the Physics 1 models and was not significant in Physics 2 models. This may have resulted from the low pretest scores in both classes making the pretest scores less predictive of success. Henderson et al. demonstrated that CSEM pretest scores were less strongly related to conceptual preparation for women than for men; they attributed this difference to the lower scores of women shifting their score distribution nearer to the pure guessing distribution [91]. A similar effect may lessen the predictive power of the pretest in the classes studied.

Gender, race/ethnicity, and first generation college-goer status were not important variables to predicting students' physics course success. Prior work has suggested that race/ethnicity and first generation status are strongly mediated by academic preparation variables such as ACT/SAT scores $[163,164]$. While there is substantial literature suggesting 
gender is an important variable for the prediction of physics post-test scores [90], there is an equally significant literature suggesting that, in general, women earn higher course grades than men [165]. Neither effect was important to the prediction of physics grades when general college-level control variables, such as CGPA, were available.

\subsubsection{Additional Observations}

Beyond the possibility of constructing accurate classification models, the logistic regression results, the variable importance results, and the LIME results provide additional insight into student outcomes. Variable importance measures show where an instructor should look to identify at-risk students and show that different measures are important at different times in a physics class. This also allows the identification of grades that are collected that do not predict student course outcomes; it may be efficacious to revisit these assignments to determine why grades on the assignment are not related to overall success in the course. Logistic regression odds ratios quantify the size of the effects of different variables. The LIME algorithm, Fig. 5.9, provides a detailed, by-student, characterization of how the models made their decision when predicting student outcomes and provides insights into where interventions could have the greatest impact. It is important to understand that the LIME results are particular to each case and should not be generalized to all students.

The conceptual pretest scores had very low variable importance in any model that included institutional variables, particularly CGPA. While this may be the result of the low pretest scores as discussed above, it may also result from the pretest providing primarily a measure of general academic ability rather than specifically physics prior preparation. This would explain why pretest scores were unimportant in models containing superior measures 
of general academic ability such as CGPA.

\subsubsection{Recommendations}

The methods explored in this paper allow any instructor to develop risk models for introductory physics classes. These models could be used to target interventions to at-risk students, possibly strongly improving STEM retention. The models are more accurate earlier in the semester if a few institutional variables are available, particularly CGPA. Departments and institutions should develop practices to make these variables easily available to instructors.

The LIME models provide a detailed student-level picture of probable class outcomes and a time-resolved evolution of the outcome along with the factors influencing the prediction. In the future, further studies may investigate whether it is productive to make these models available to the students.

\subsubsection{Limitations}

Even with both in-class and institutional data, the models were not completely accurate. Analysis of the confusion matrices show a bias toward false positives in many of the models. The decision threshold should be determined on a case-by-case basis depending on instructor comfort with misclassification. These models may not be entirely generalizable as they depend on the local educational environment at the institution studied. Because of this, the techniques for constructing the models should be adopted instead of the individual models when extending to different environments. 


\subsection{Conclusions}

Machine learning techniques produced accurate predictions of student course outcomes. Models using institutional data were more accurate before the class began than models using only in-class variables were in the second week of class in Physics 1, the fourth week of class in Physics 2. The accuracy of the models varied between the two courses studied; however, many of the same variables were common to the optimal models for the two courses. By the first examination in both courses, the institutional variables no longer improved the models. The importance of CGPA as a measure of preparedness was reinforced in this study with none of the additional institutional variables near the level importance of this single measure. Using the LIME algorithm, it was possible to show which factors were most important for each student in predicting his or her most likely course outcome. The combination of these models and the LIME algorithm produced a highly accurate and detailed classification of student risk that can be used to target interventions at the student level in introductory physics. 


\section{Part III}

\section{The Pulsar Search Collaboratory}




\section{Chapter 6}

\section{Introduction to Pulsar Search Collaboratory}




\subsection{Introduction}

The Pulsar Search Collaboratory (PSC) is a distributed authentic science project designed to inspire high school students to consider STEM careers by providing them with the opportunity to conduct actual scientific work analyzing radio astronomy data to discover pulsars. Students involved in the PSC analyze data collected from the Robert C. Byrd Green Bank Telescope (GBT) in Green Bank, West Virginia and then use the same tools designed and used by actual astronomers to evaluate pulsar candidates. The PSC has been running continuously since its pilot program in 2008 and starting in 2016 transitioned to an onlinefocused model. Over 2500 students and teachers have participated in the PSC and have discovered 7 pulsars and one rotating radio transient since 2008 [166].

\subsubsection{The Original PSC}

The original idea for the PSC was developed in 2008 while the GBT was undergoing a track replacement. During this time, the telescope was parked, with the sky allowed to "drift" overhead. Approximately 300 hours of Drift Scan Survey data were collected resulting in approximately 16,500 pseudo-pointings. These data represented an almost completely unobserved part of the sky at this frequency and had the potential for numerous discoveries; however, each pointing would need to be manually inspected in order to determine if a pulsar was present. Instead of having trained astronomers, astronomy graduate students, or upper division undergraduates evaluate the data, it was decided that this data was to be used to engage high school students in an authentic science activity [167].

Though a novel outreach application, the PSC is not the first of its kind to attempt to 
engage the public in scientific research. Persons interested in getting involved in the search for extraterrestrial intelligence can lend computational time from their home computers through the SETI@home program [168]. For people looking for greater interaction with the research, the Galaxy Zoo program allows members to evaluate optical images of distant galaxies and help to classify the different types of galaxies. The Galaxy Zoo model has been expanded to dozens of other projects including evaluating climate change data, looking for exoplanets, and transcribing ancient documents, all through the zooniverse portal. [169]

While projects like zooniverse have focused entirely on online involvement of individuals, the PSC pursued a separate course. In its initial release, the program focused on robust inperson interactions between researchers, students, and teachers. Educators in West Virginia and the surrounding states were approached to start PSC clubs at their schools where interested students would work together in collaborative units. These individual clubs would then receive online mentorship from astronomers and astronomy graduate students. Representatives from these clubs would be able to attend a PSC summer camp with their teacher prior to starting the program at the GBT to learn how to evaluate pulsar data and take that knowledge and excitement back to their clubs for the year. Students who were very active in the PSC would be eligible to attend a capstone event at WVU where they would have the opportunity to present their work in a poster session and meet other PSC participants from other schools. The formalized collaborative environment unique to the PSC was developed to foster a sense of community for the students and educators. This community would allow participants to rely on one and other and draw support from the broader group with the goal of keeping these students in the STEM pipeline. 


\subsubsection{The New PSC}

Starting in 2016 the PSC transitioned to a new model shifting from in-person interactions to a distributed online model. Like the Original PSC, students would still work as part of clubs or teams at their school while supervised by a teacher and would continue as before to analyze pulsar data with the professional tools. However, in order to facilitate nationwide involvement, a series of "hub institutions" were recruited to serve the roll played by WVU in the original program. These hubs were responsible for recruiting teachers to start PSC clubs at their schools and would provide the astronomy expertise through undergraduate and graduate student mentors. The hubs would then serve as the locus of New PSC communities and would host regional capstone events around the nation. While distributed in this hub model, all of the students and teachers participating in the PSC would work through a central online portal.

Through the online portal, students would be able to interact with each other, their mentors, hub astronomers, and the staff at Green Bank. Students and teachers would receive their training through live online events and, as people progressed through the program, be able to participate in live learning sessions, office hours, and group pulsar scoring with participants around the nation. To quickly transition to this model, the existing web infrastructure was adapted to this new role with the belief that modification and improvements could be added as needed throughout the duration of the New PSC.

The education research component of the PSC seeks to determine how the PSC experience affects the science identity and STEM career intentions of its participants. These questions were investigated by comparing pre-survey and post-survey results and by examining the 
participant's interaction with the PSC online portal.

In order for students to become fully certified to participate in the PSC, they must complete several steps. First, they must join a "team" set up by their teacher and register with the program. Once registered there are multiple training videos and documents for the students to review to learn how to properly identify pulsars from the data. The program provides a 6 week online course with weekly lectures for students. Once students have fully reviewed these materials on their own or as part of their school's PSC club, they are required to complete two sub-tests of their pulsar grading skills to become fully certified to score pulsar plots as members of the PSC. This process requires them to pass both tests with perfect scores, but does not limit the number of attempts. Once they have passed both tests, they can begin to examine and score original pulsar data in an attempt to discriminate pulsars from noise. Students who score a sufficient number of plots are eligible to participate in a capstone event or to attend PSC summer camp.

Teachers use the PSC in many ways. Some form optional afterschool clubs, some use the data and scoring activity as part of their curricula, and some use the data to support student participation in other science activities.

\section{Measures}

The PSC survey examines participants' STEM intentions along a number of dimensions: Science/Engineering Identity, Self-Efficacy, Science Career Interest, and Sense of Belonging. Each of these domains were probed using well validated, pre-existing instruments. The domain of Science/Engineering Identity was probed using elements of the Persistence Research in Science and Engineering (PRiSE) survey developed by Hazari et al. [170] and work by 
Perez, Cromley and Kaplan [171] and Aschbacher, Li, Roth [172]. Items related to SelfEfficacy were derived from the work of Glynn et al. [173] as well as Trujillo and Tanner [174]. Belonging was examined using the work of Trujillo and Tanner [174] and the study of Belonging in Youth Development Programs by Anderson-Butcher and Conroy [175]. Finally Science Career Interest used items from STEM-CIS by Kier et al. [176] and the STEM Career Interest Questionnaire by Tyler-Wood, Knezek, and Christensen [177].

An Exploratory Factor Analysis (EFA) was conducted which recovered these facets within the survey data. Further, EFA within the individual sub-scales showed the Science/Engineering Identity, Self-Efficacy, and Science Career Interest sub-scales as single factors while the Belonging sub-scale separated into three sub-factors: Recognition (by teachers, mentors, etc.), Belonging Amongst Peers, and Negative Feelings. Confirmatory factor analysis demonstrated that the belonging sub-scales were robust producing a Comparative Fit Index (CFI) of 0.96. In the following sections, the New PSC is compared to the Original PSC. We will investigate the level of student engagement with the new, online-focused, system and illustrate what was successful and what future programs attempting such a transition can expect. Additionally, the student perception of the New PSC was investigated. Finally, an analysis of how the PSC influences affective variables linked to success in college STEM programs such as belonging, self-efficacy, science identity, and science career intentions were evaluated. 


\section{Chapter 7}

\section{Programmatic Evaluation of the New PSC}




\subsection{Student Engagement}

One goal of the PSC project was to determine if an online implementation could provide an experience similar to that of the previous face-to-face experience. To evaluate this from the programmatic side, we looked at several metrics of student engagement with the New PSC versus the Original PSC. These will be compared holistically, treating the Original PSC as data collected from 2009 through 2012 (2008 was removed due to incomplete data collection) and the New PSC as data collected from 2015 through 2018. These will also be disaggregated into yearly data sets starting October 5 and ending on May 15 (i.e. the 2010 distribution starts October 52010 and ends on May 15 2011). During the interim years of 2013-2015, there was no formal PSC program. Although students and teachers did continue to score plots during this time, for the sake of comparison, this interim period will be excluded from most of the analysis.

In terms of pure participation totals, the Original PSC far outperformed the New PSC with an average number of 591 participants per year between 2009 and 2013 compared to an average of 165 in the New PSC between 2015 and 2017. Unlike the Original PSC, the new version experienced much more stable participation with the largest year to year fluctuation being an increase of 58 students between 2015 and 2016 or an approximate increase of $38 \%$. Conversely, the Original PSC experienced steady growth until 2011 with the largest increase in participation between 2010 and 2011 of 341 or approximately $69 \%$, stabilizing at around 830 participants. The lack of growth in the New PSC likely indicates that the participation near the current 165 participants is what can be expected going forward assuming no programmatic changes. 


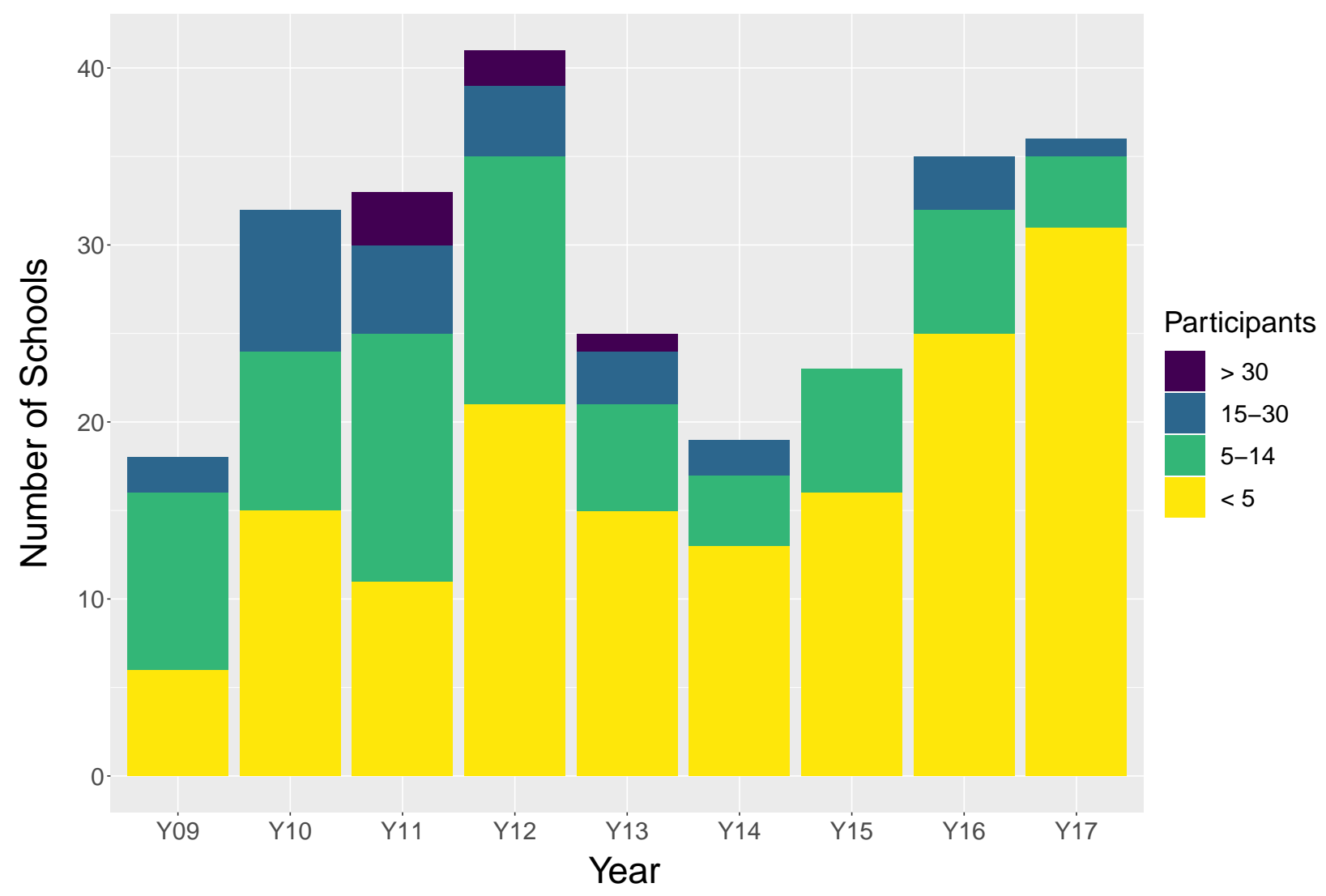

Figure 7.1: Participating Schools Based on Active Members: The number of schools participating in the program each year of the PSC disaggregated by the number of active members in the program in each of these years.

\subsection{School Participation}

Overall numbers do not tell the complete story of the PSC. In order to better understand the program and where it is succeeding and where it needs improvement, it is necessary to look at the granular by-school data.

Figure 7.1 shows that despite the lower overall participation of students in the New PSC, the overall participation of schools has recovered to nearly that of the Original PSC with approximately as many schools participating in 2017 as any year of the Original PSC. However, the New PSC is dominated by clubs with fewer than 5 active participants whereas the Original PSC had a greater distribution of clubs with more active students with around 


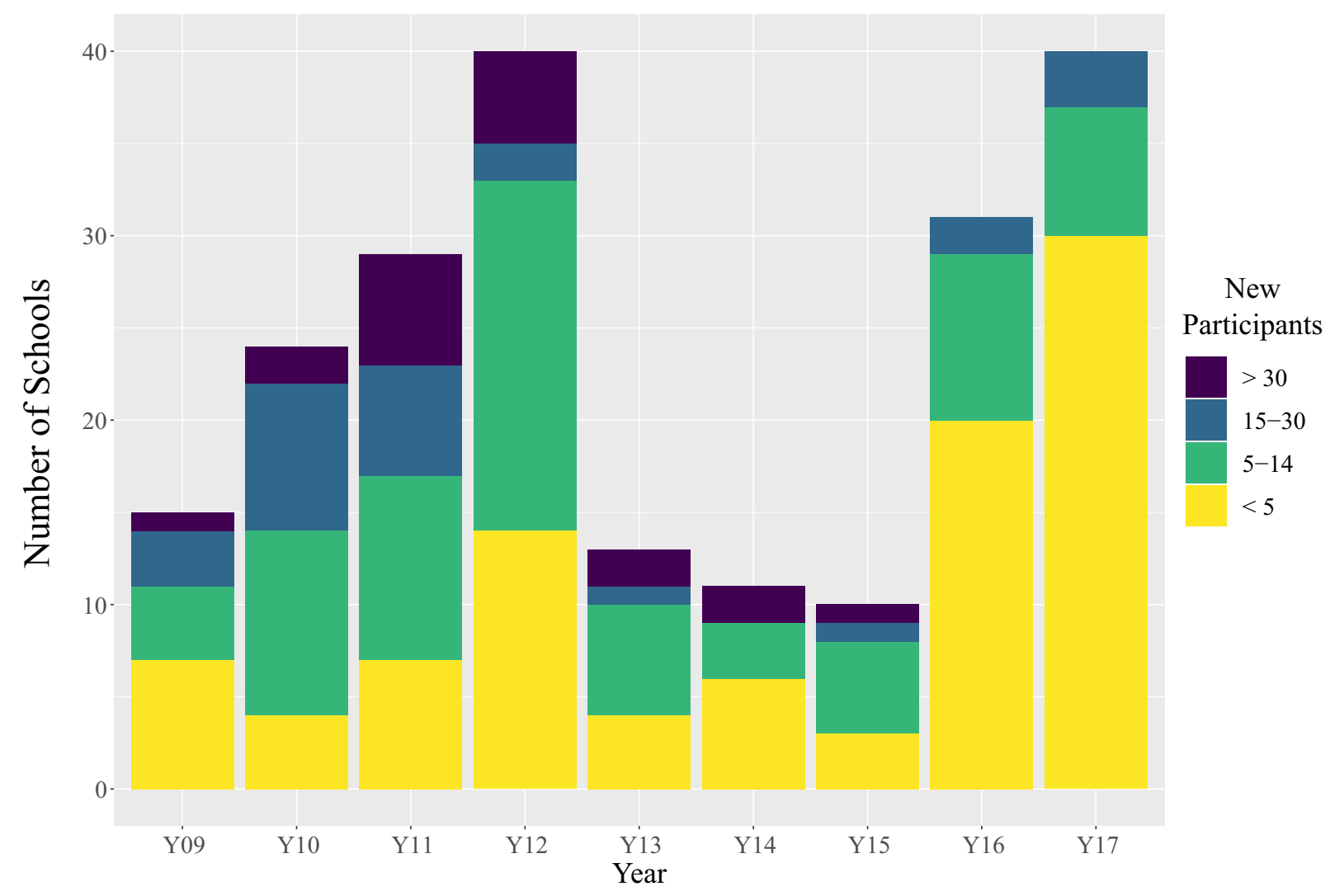

Figure 7.2: Participating Schools Based on New Members: The number of schools participating in the program each year of the PSC disaggregated by the number of new members joining the program in each of these years.

half of all clubs registering greater than 5 active members.

In terms of recruitment as shown in Fig. 7.2, there is parity in terms of the overall number of schools adding new members. As with the active participation rates, the most common club in the New PSC adds fewer than 5 new members annually as compared to the Original PSC where it was far more likely that a club would add between 5 and 14 new members.

When only new members who were active (defined as having scored at least one plot, but not necessarily passing any of the sub-tests) were examined in Fig. 7.3, there exists similar parity between the Original and New PSC when just new members were considered. 


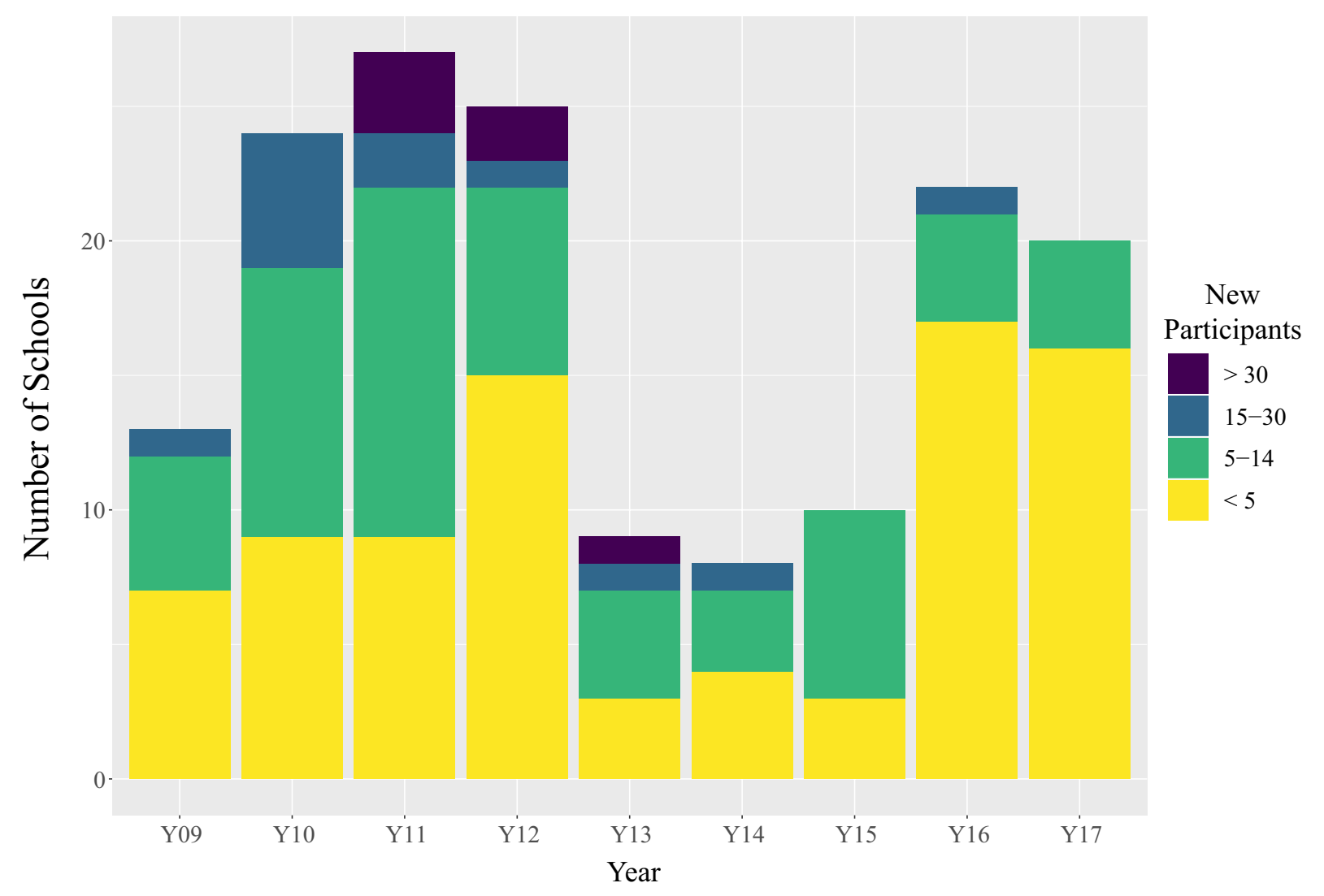

Figure 7.3: Participating Schools Based on Active New Members: The number of schools participating in the program each year of the PSC disaggregated by the number of active new members joining the program in each of these years.

At their peaks, year 12 (Y12) for the Original PSC and Y17 for the New PSC, the Original PSC has 25 participating schools and the New PSC has 20. When schools with greater than 5 new members are removed Y17 actually had more participating schools than Y12.

This indicates that one possible reason for the success of the Original PSC in terms of participation was a small number of large clubs along with a significant number of clubs which sustained above 5 active members per year. Clubs which are routinely able to recruit significant numbers of new members are likely the most well organized, well led, and galvanized by the program. When these large programs are ignored, the New PSC appears to be functioning similarly if not better than the Original PSC with a much higher 
number of small clubs in terms of new members added and a similar number in terms of active new members. Despite the transition to a nationwide program scheme, the PSC is still only seeing similar school participation to what was seen in the original regional program, though more geographically distributed.

\subsection{Capstone Qualification}

One of the major components of the program for PSC students was the capstone event at the end of each year. In order to qualify, students were required to complete 50 pointings or 1500 plots scored. In 2017, the requirement was dropped to 20 pointings to encourage greater participation in capstone; however for the sake of comparison, 50 pointings will be used as the official threshold for all of the following analyses.

As can be seen in Fig 7.4, the proportion of total participants qualifying for capstone decreased dramatically from the Original PSC (weighted mean qualification rate of 36.1\%) to the New PSC (weighted mean qualification rate of $17.8 \%$ ) along with overall rate of participation. It is worth noting that even in the Original PSC only around one third of participants reached the capstone level. This may indicate that current low levels of reaching capstone may be in part be caused by the same issues related to the role of achieving capstone in the Original program. Additionally, the New PSC capstone rate increases annually from $12.4 \%$ in Y15 to $25.7 \%$ in Y17. While participation in the program is an important programmatic aspect of the PSC, it is also important to evaluate the degree to which students are participating in the central aspect of the program, scoring pulsar plots. There exist several outliers within the pulsar scoring data of students who scored tens of 


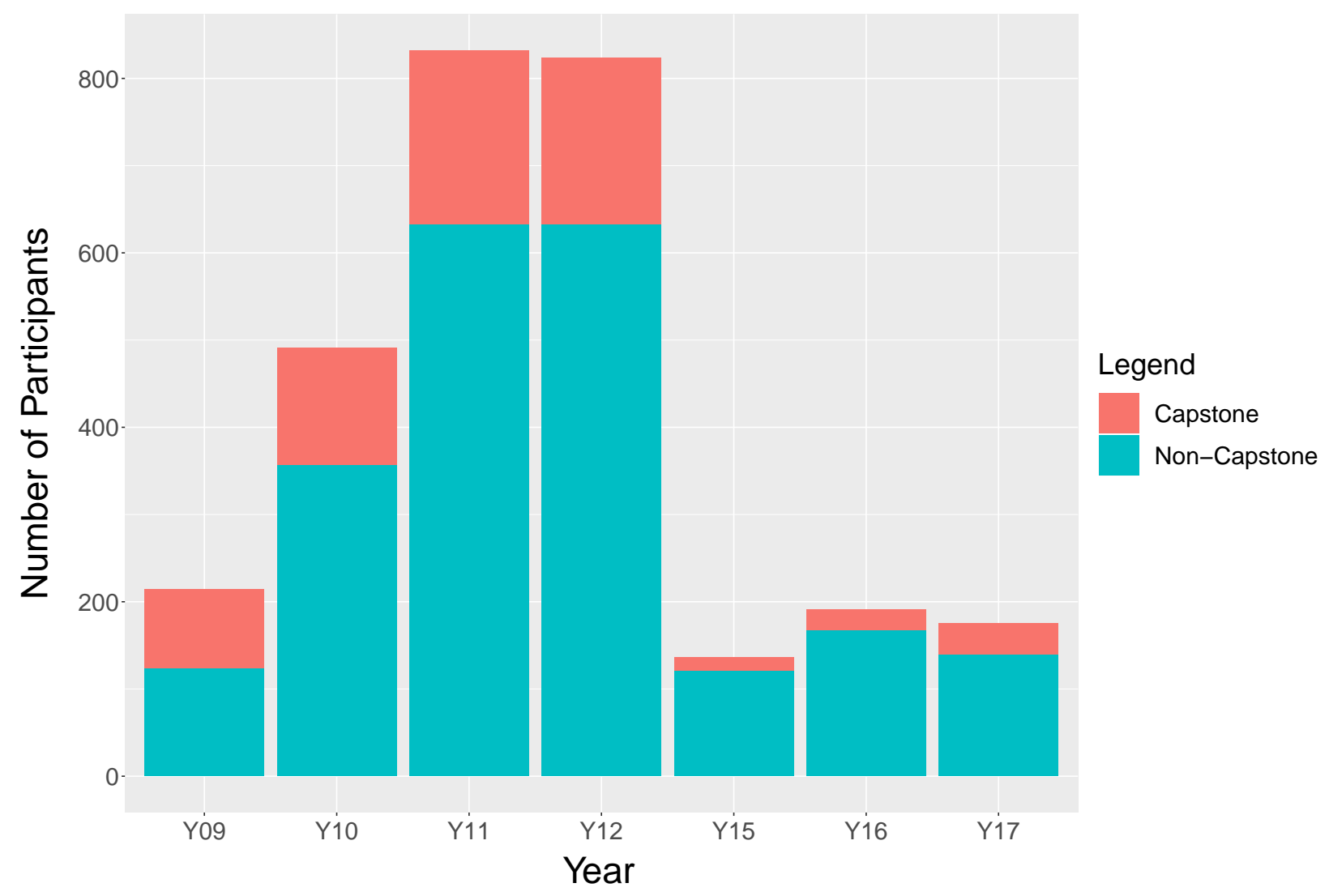

Figure 7.4: Total Participating Members By Year and Capstone Qualification 


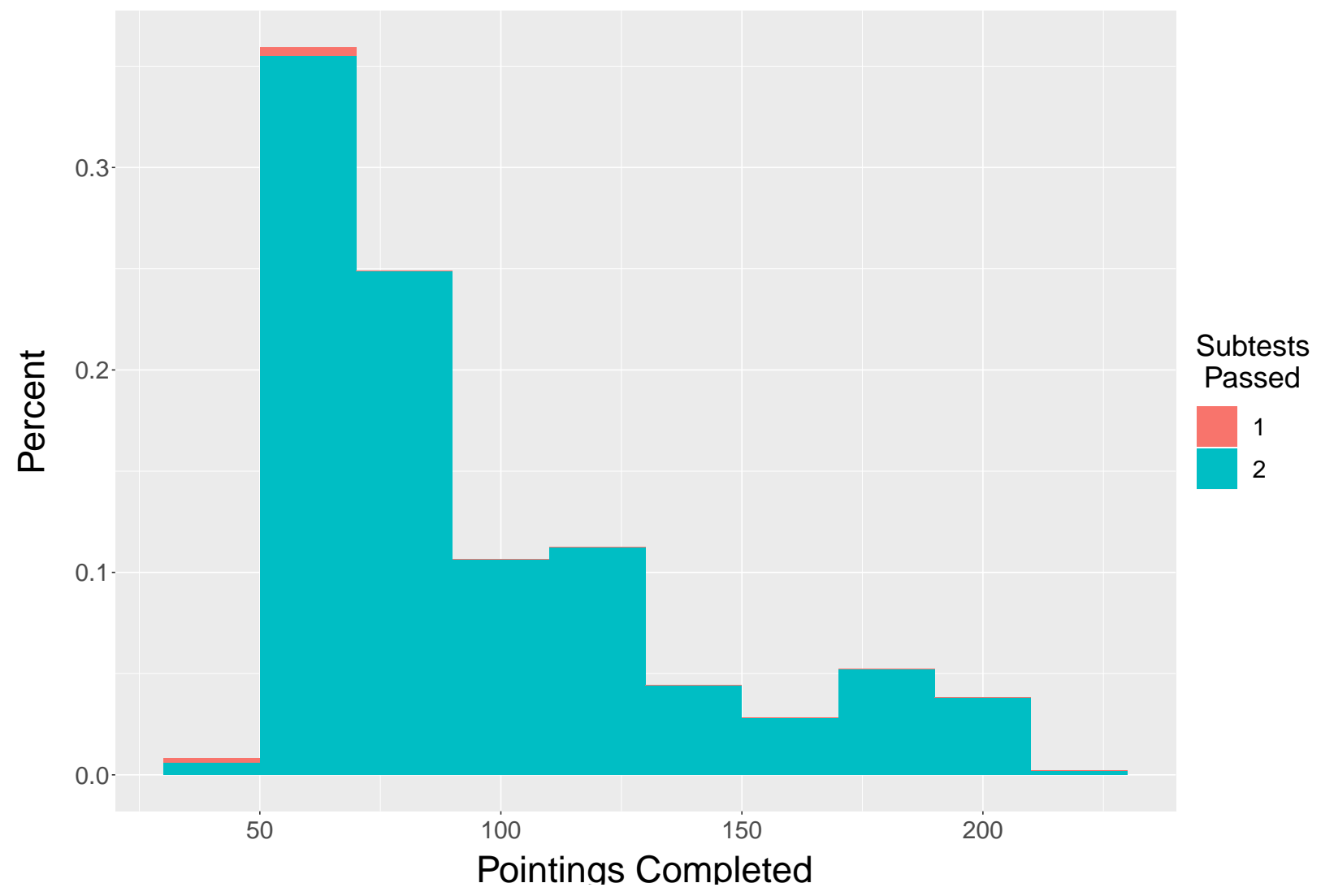

Figure 7.5: Pulsar Candidate Pointings Completed by Students Who Qualified for Capstone in the Original PSC - Lower 95\% of Plot Scorers

thousands of plots. These individuals were filtered out of the general analysis of the program; the data were restricted to the lower $95 \%$ of plot scorers, leaving 2721 participants in the data set.

Figures 7.5 and 7.6 show the distribution of pointings completed by students who qualified for capstone in both the Original and New PSC. In both cases, the largest group of students were situated at the capstone qualification point, indicating that capstone qualication was a potential motivating factor for these students. However, in the Original PSC the positive tail of the distribution is larger, showing that more students proportionally continued with the program after qualifying for capstone, which may indicate that the program was more engaging for students in the Original PSC. Additionally, the Original PSC contains 


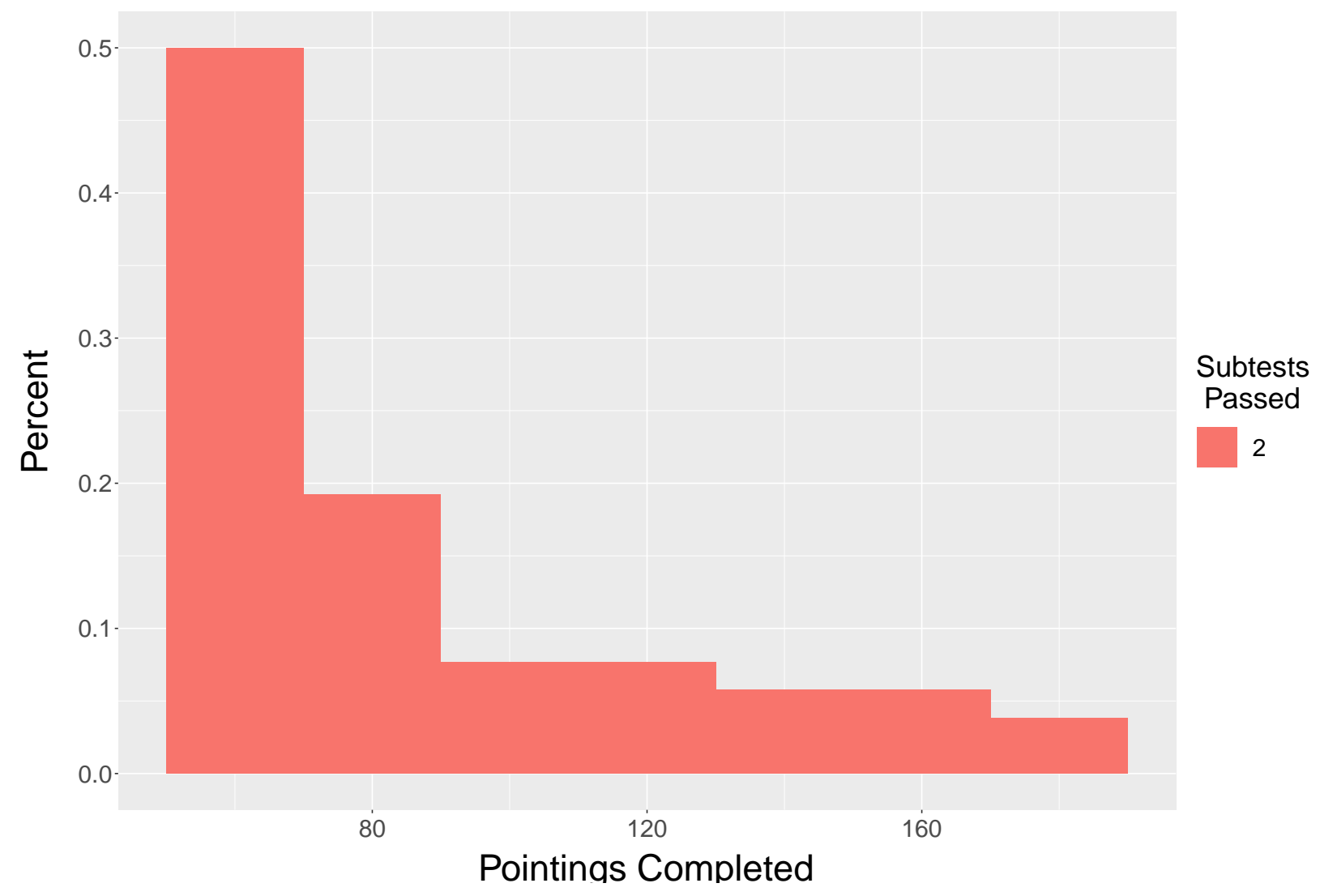

Figure 7.6: Pulsar Candidate Pointings Completed by Students Who Qualified for Capstone in the New PSC - Lower 95\% of Plot Scorers

a small number of students who scored enough plots to qualify for capstone, yet did not complete all of the requisite subtests as can be seen in Fig. 7.5. This was not seen in the New PSC, indicating that for some period in the Original PSC the online testing software contained errors.

When the students who did not qualify for capstone are evaluated in Fig. 7.7 and Fig. 7.8, the distributions are strongly peaked around zero pointings completed in both versions of the PSC. This indicates that for students who do not qualify for capstone, most will abandon the program early in their experience. This provides further evidence that the capstone threshold serves as a strong motivator and milestone for students engaged with the program. Furthermore, unlike the capstone-qualified students, all of the participants who 


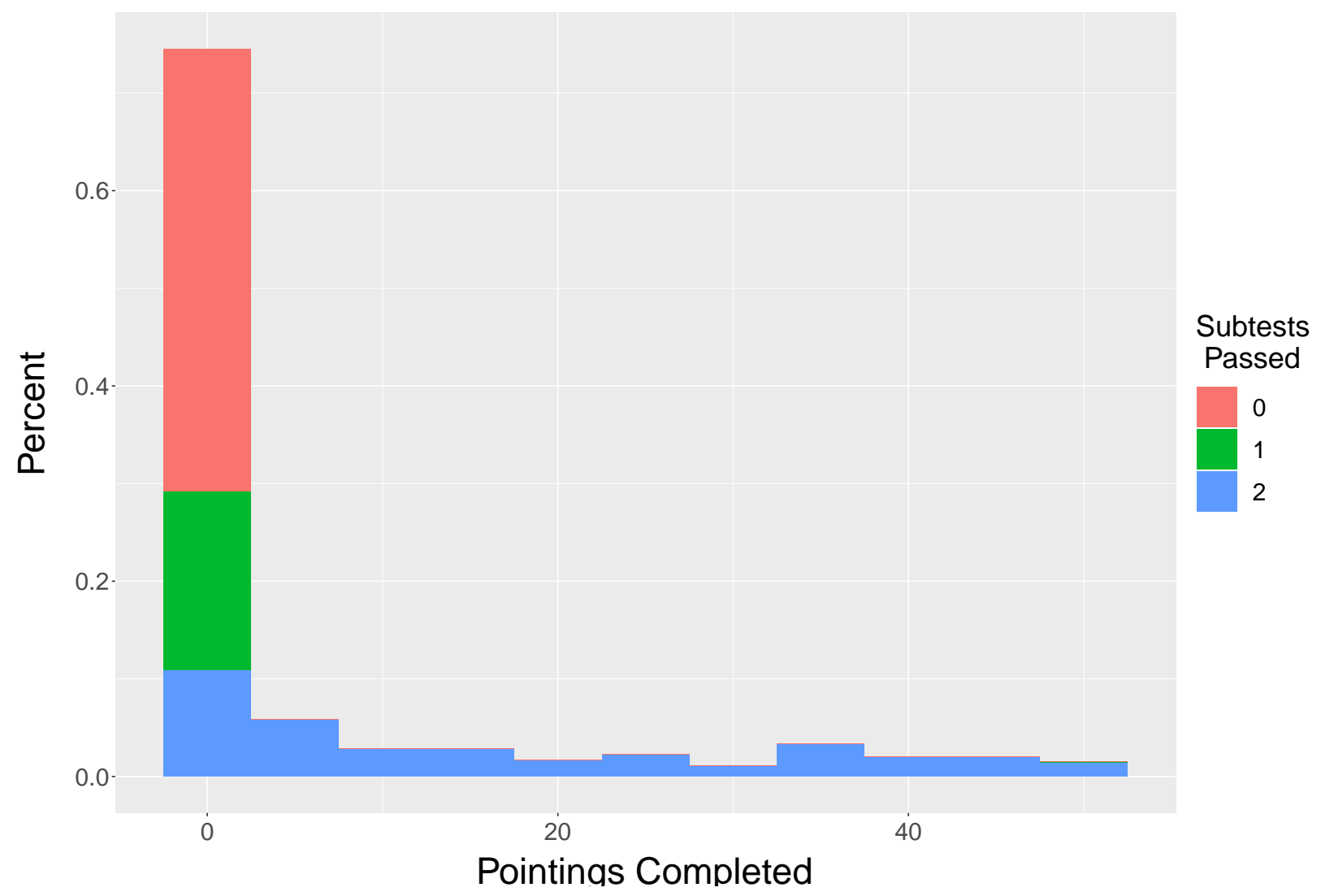

Figure 7.7: Pulsar Candidate Pointings Completed by Students Did Not Qualify for Capstone in the Original PSC - Lower 95\% of Plot Scorers

do not pass subtest 2 are in the lowest bin for pointings completed.

Figure 7.9 shows the density plots by year of the pointings scored by capstone qualifying full PSC participants, defined as having passed both subtests. In each of the years of the Original PSC, the results are strikingly similar, with most students localized around the capstone threshold with a thick tail of students continuing beyond that point. Conversely the New PSC is very diverse in its three year run. Y15 is anomolous in that there is no clear peak, but instead a relatively uniform distribution between 100 and 300 pointings. This may be a result of being the first year of the new program, but we lack the data necessary to compare to the first year of the Original PSC in 2008. It may also be that as the first year of this version, the teachers and students participating did not have a clear picture of the 


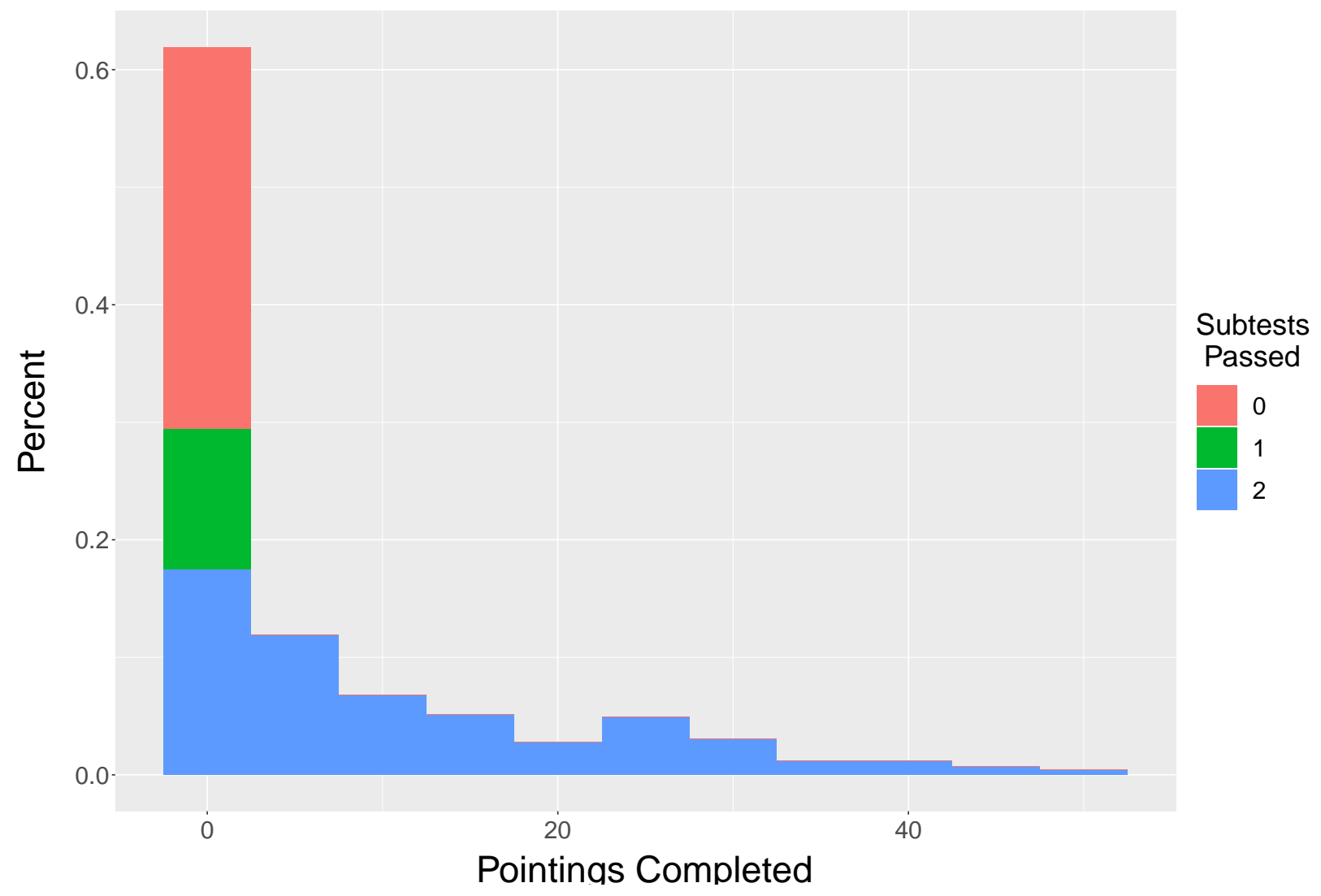

Figure 7.8: Pulsar Candidate Pointings Completed by Students Did Not Qualify for Capstone in the New PSC - Lower 95\% of Plot Scorers

capstone event and it served as less of a motivator than in previous or subsequent years. This may be the case as by Y16 the distribution is very similar to that of the Original PSC and by Y17 the distribution is almost entirely skewed toward the capstone qualifications.

For those students who did not reach the capstone threshold as shown in Fig. 7.10, most years of the PSC are very similar. In Y10 and Y15 there were a greater proportion of students completing fewer pointings than in other years. In the final two years of the Original PSC, Y11 and Y12, the distributions are bimodal in character with a second peak slightly less than the capstone threshold. This indicates that there existed a cohort in the Original PSC that made a significant effort to reach capstone, but did not succeed, and that this cohort does not seem to exist as strongly in the New PSC. 


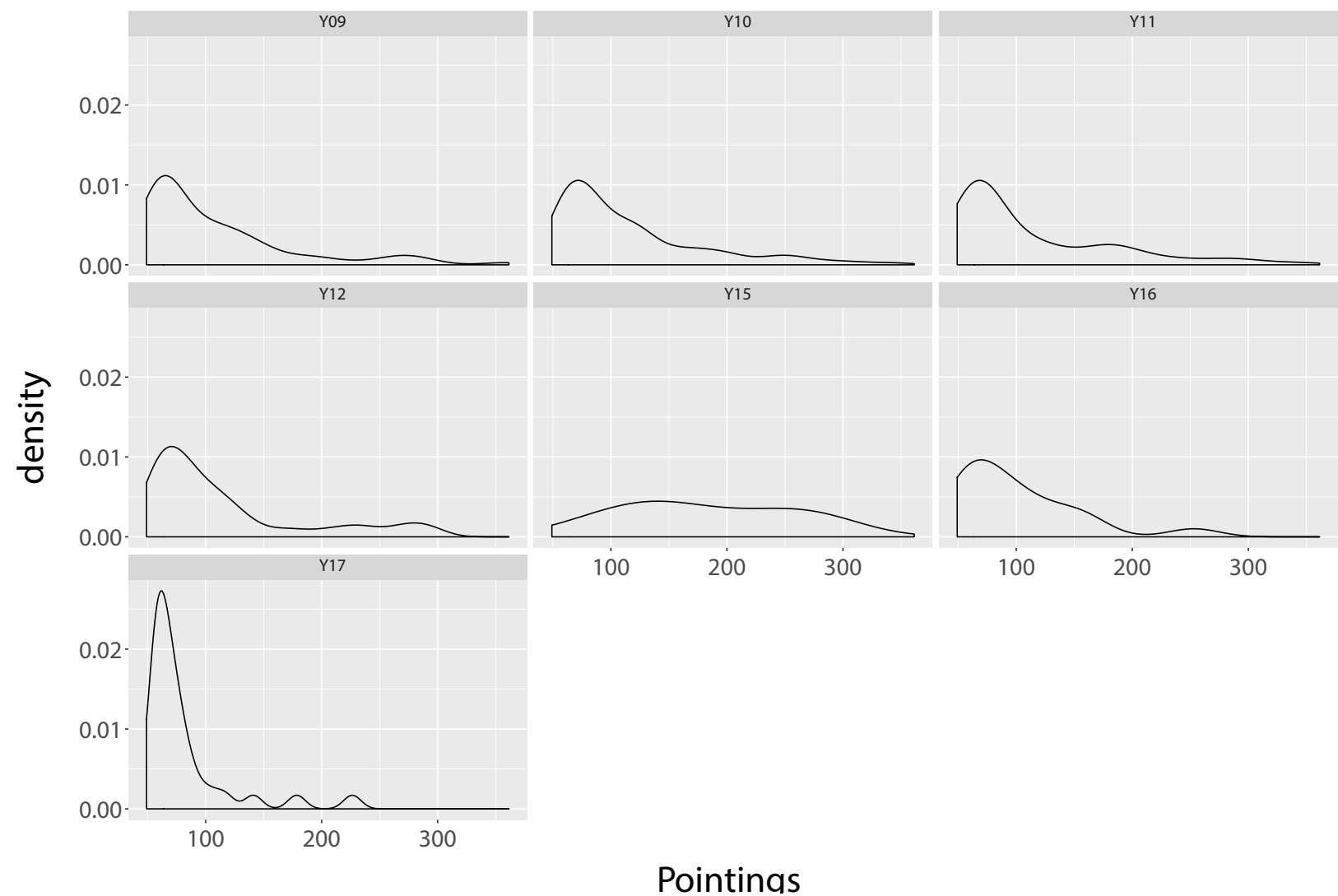

Figure 7.9: Pulsar Candidate Pointings Completed by Students Who Qualified for the PSC and for Capstone by Year - Lower 95\% of Plot Scorers. The y-axis for each is the proportion of students participating in that year and the $\mathrm{x}$-axis represents the number of pointings scored

The New PSC has far fewer students than the Original PSC which can effect the interpretation of the density plots of the capstone participants. It does not appear as though the lowering of the requirement for capstone to 20 pointings in Y17 had any effect on the number of pointings done by the students; however, this could also have resulted from incomplete communication of this change to the participants.

\subsection{The Qualification Tests}

The New PSC outperforms the Original PSC in the proportion of students who pass both subtests to qualify to fully participate in the program. Figure 7.11 shows student 


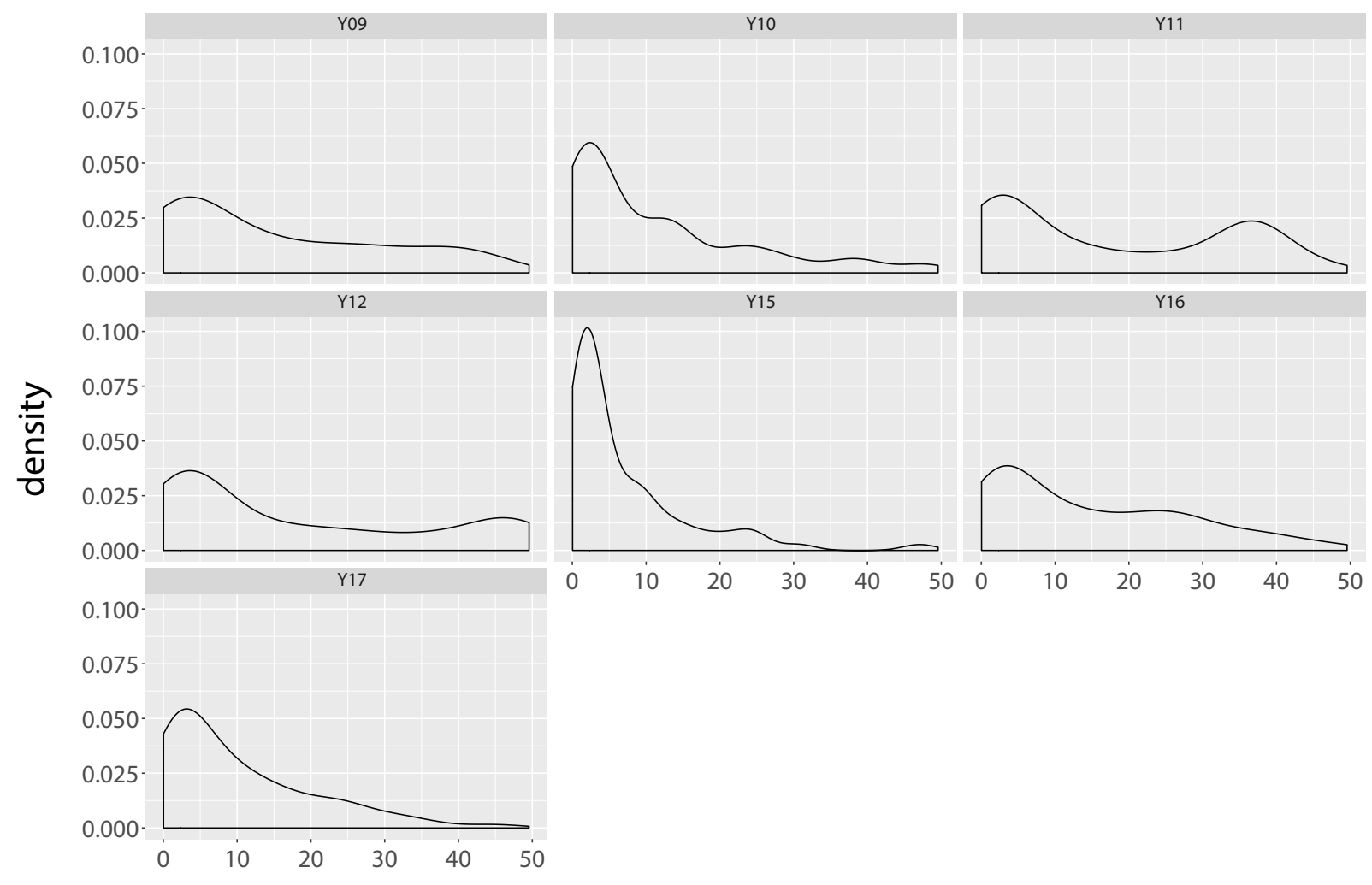

\section{Pointings}

Figure 7.10: Pulsar Candidate Pointings Completed by Students Who Did Not Qualify for the PSC and for Capstone by Year - Lower 95\% of Plot Scorers

subtest passage by year. In years Y10-Y12 of the Original PSC, only around one half of students completed subtest 2 and a substantial proportion only completed subtest 1 . In both Y11 and Y12, around 100 students were sufficiently engaged with the program to pass the first subtest, yet did not finish the final subtest. Both versions of the PSC show a large number of students not matriculating through the subtest structure toward full participation.

Based on subtest passage, one third to one half of all students who signed up for the PSC did not persist through the subtests as seen in Table 7.1. This led to further investigation through interviews with teachers and students that showed that the current certification structure requiring the students to score $100 \%$ on each of the certification tests served to dissuade students from persistence in the program. Steps have been taken to modify the 


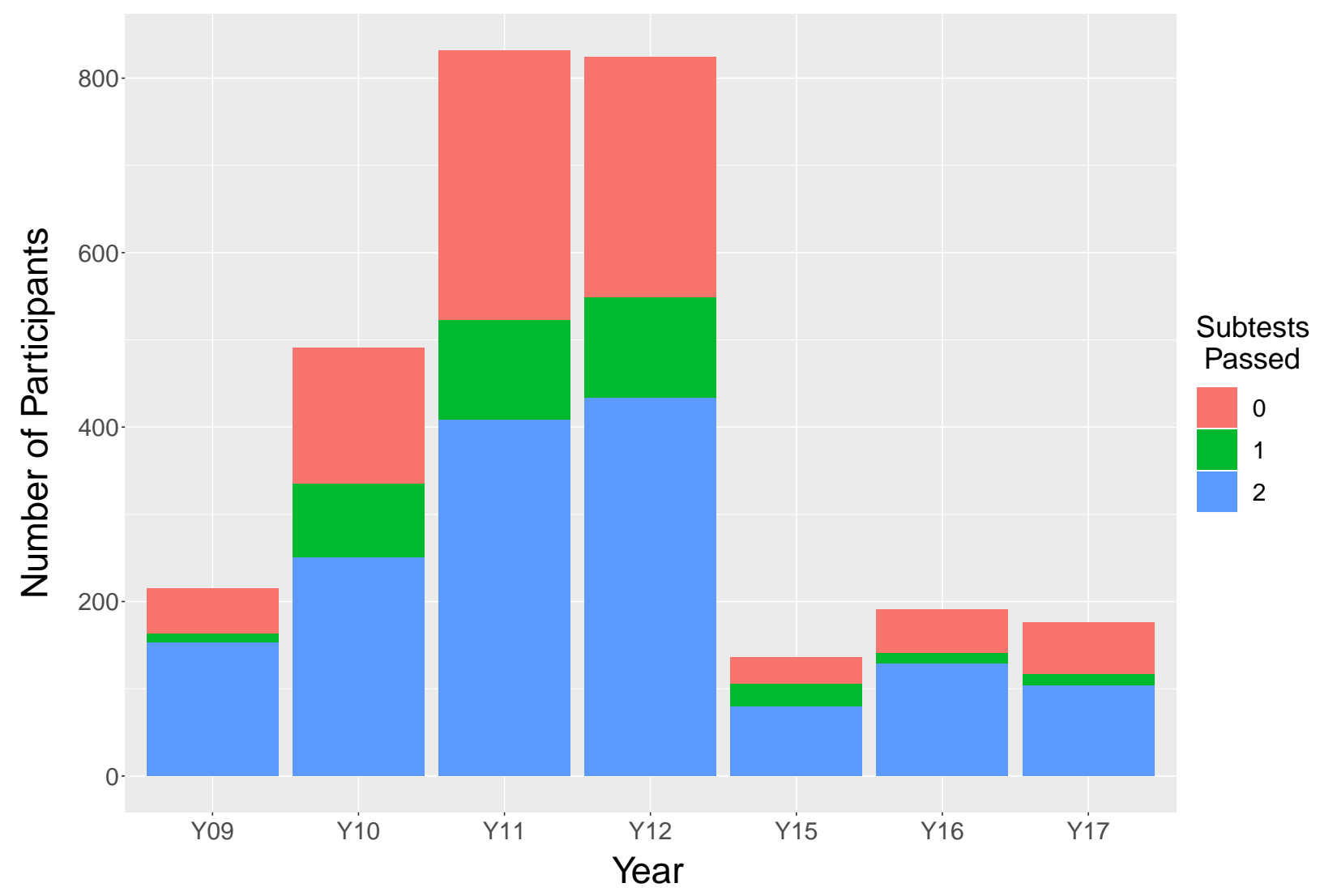

Figure 7.11: Total Participating Members By Year and Number of Sub-Tests Passed: In order to qualify to fully participate in the PSC members must complete both subtest 1 and subtest 2. Only student with classification 2 should be considered full participants in the PSC.

testing process to improve student persistence by switching to a multiple-test process for "Pulsar Certification." Each of these sub-tests will gradually increase in difficulty, building up student confidence and understanding while serving as a gateway to participation instead of a barrier. Additionally, the training videos have been edited into shorter and easier to digest pieces and more online interaction have been added to compensate for the loss of in-person training from the Original PSC years.

One can examine the negative effect of the current testing structure by plotting the number of times the students attempted the test. Students are divided into four groups: those who passed no subtest, those who passed subtest 1 but did not attempt subtest 2, 


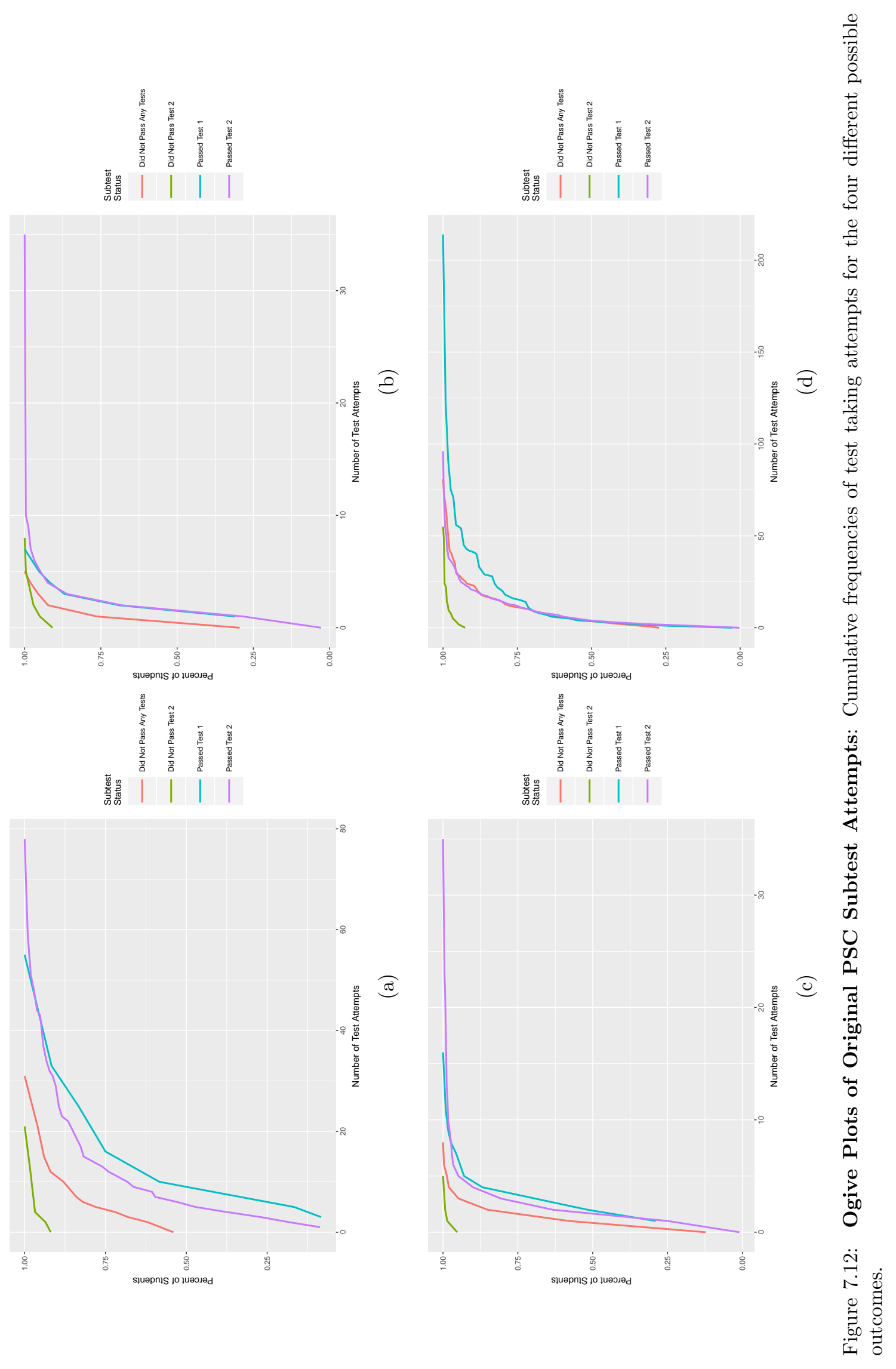



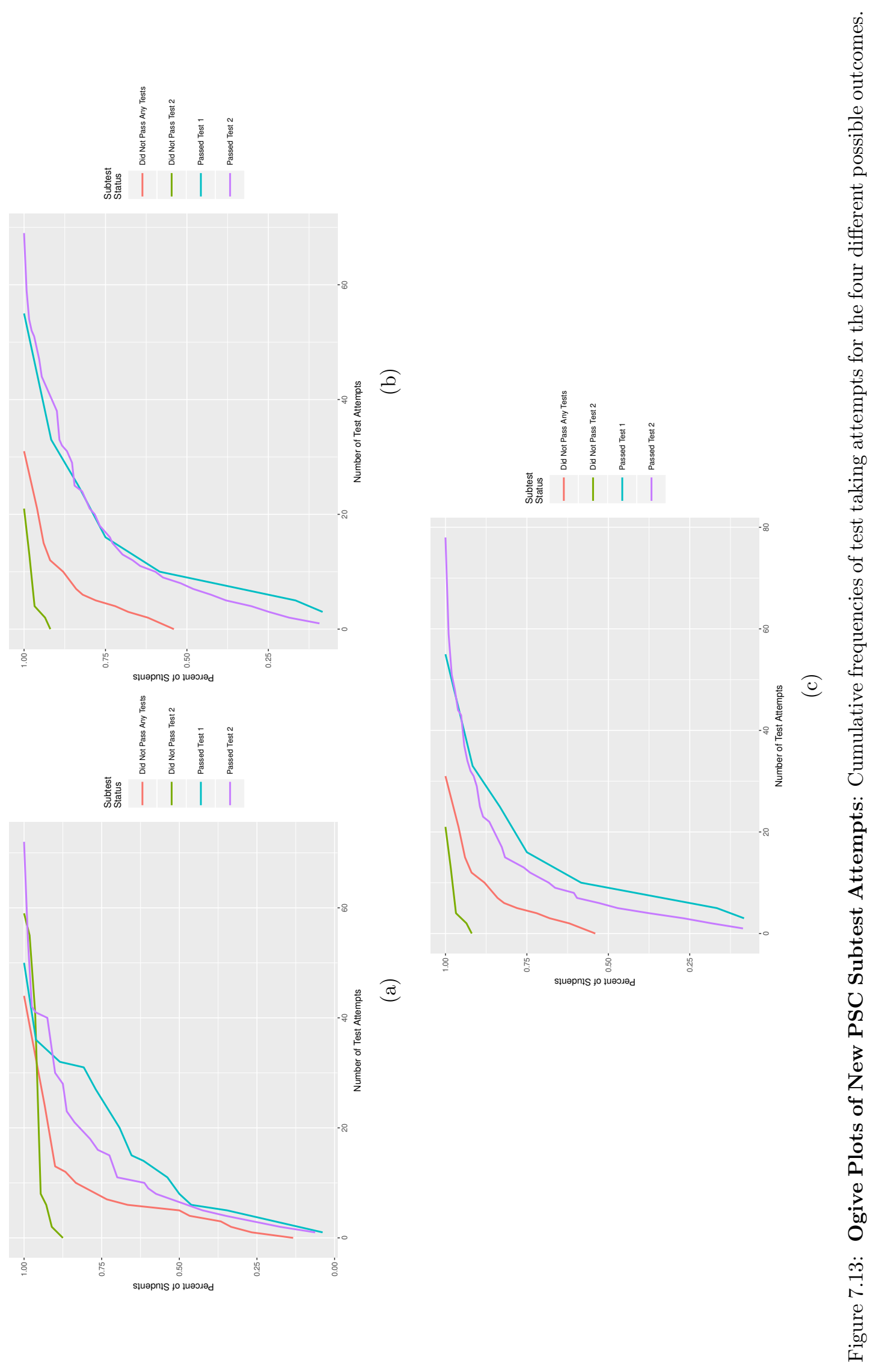


\begin{tabular}{|c|c|c|c|}
\hline Time Frame & N & Percent Pass Subtest 1 & Percent Pass Subtest 2 \\
\hline $2009-2010$ & 215 & 76 & 71 \\
\hline $2010-2011$ & 491 & 68 & 51 \\
\hline $2011-2012$ & 832 & 63 & 49 \\
\hline $2012-2013$ & 824 & 67 & 53 \\
\hline $2015-2016$ & 136 & 78 & 59 \\
\hline $2016-2017$ & 191 & 74 & 68 \\
\hline $2017-2018$ & 176 & 66 & 59 \\
\hline
\end{tabular}

Table 7.1: Percentage of total students who pass either subtest 1 or subtest 2 .

those who did not pass subtest 2, and those who passed subtest 2. Students who passed subtest 2 were qualified for the primary activity of the PSC, searching for pulsars. Figure 7.12 give examples of the number of attempts required to become certified in the original implementation of the PSC. Cumulative distribution of number of tests taken before passage shows the number of attempts for $50 \%$ of students to pass the tests varied from as low as 2-3 to 10 or more. Figure 7.13 provides the same information for the New PSC. In the New PSC, the number of required attempts for students to pass the sub-tests is much higher than in the peak years holding steady around 10 attempts, but with a much more extended distribution with a non-negligible number of students taking the tests more than 20 times. This seems to indicate that, while the switch to the online format did not damage persistence, the online training may not function as efficiently as the face-to-face training. 


\title{
Chapter 8
}

Evaluation of the New PSC's Effect on Student STEM

\author{
Intentions
}


The second part of the research into the effectiveness of the PSC relates to students' perceived experience with the program. To ascertain the effect of the PSC on student science self-efficacy, science identity, and STEM intentions, students were presented with a two surveys. These surveys used established psychometric scales to measure the aforementioned affective qualities as well as programmatic experiences with the program. These same surveys, less the programmatic questions, were also administered to high school science students unaffiliated with the PSC to serve as a control group. This control group was comprised on high school students at the same schools as many of the PSC participants, but a complete demographic distribution of these students was not collected. The following section will summarize the feelings of PSC participants about the program and show the how the participants self-efficacy, science identity, and STEM intentions change over the course of the PSC and how they compare to that of an average high school student.

The PSC post-survey contains 27 items to gauge the students' experience with the PSC. Through these we can begin to understand how students who successfully complete the program view their experience. Figures 8.1 through 8.3 each represent a single block of questions asked on the PSC post-survey containing several items related to the student's experience on a 5-point Likert scale.

Responses in Fig. 8.1 show a negative skew (a long tail in the negative direction) in items related to student enjoyment of the PSC with very few students answering "disagree" or "strongly disagree." On average, items in this block unrelated to parents and/or teachers encouraging participation in the PSC, $55.4 \%$ of participants answer "agree" or "strongly agree" with only $8.4 \%$ answering "disagree" or "strongly disagree." The items related to parental or instructor encouragement to participate in the PSC are both positively skewed 


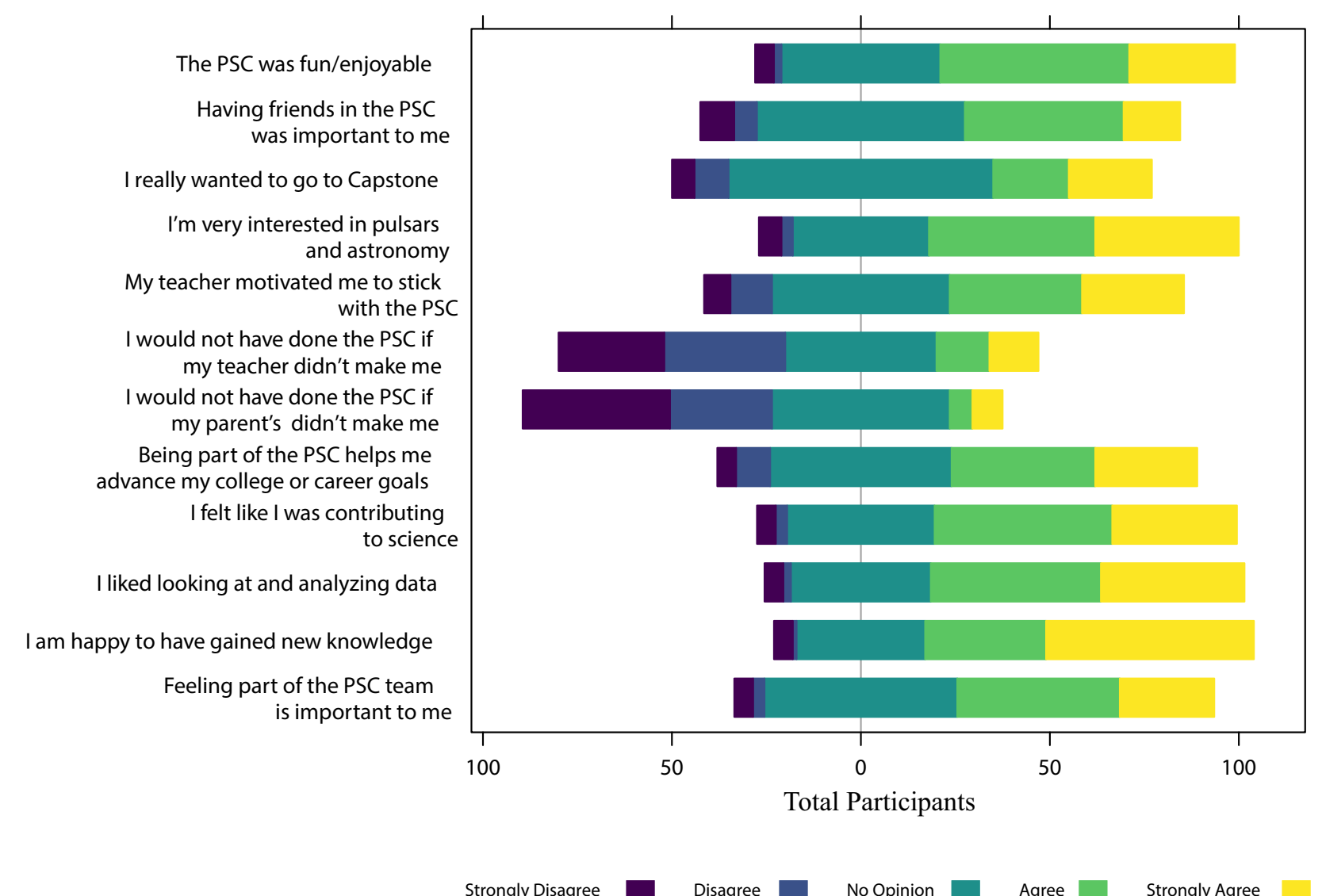

Figure 8.1: PSC Post Survey Programmatic Responses Part 1

with similar, but reversed, behavior to the other items in this block; $47.2 \%$ of students indicated that they would have participated in the PSC without being made to by a teacher and $51.9 \%$ without being made to by a parent. Instead it appears that students who persisted to the post-survey were by and large self motivated participants.

The second question block summarized in Fig. 8.2 relates to participant interaction with college non-faculty mentors. Overwhelmingly participants indicate "No Opinion," with an average of $56.4 \%$ of participants choosing this answer. For items related directly to interactions, this result is not surprising. Interviews conducted throughout the New PSC have indicated that many participants do not have regular interaction with either mentors or astronomers and in many cases the participants have only a single interaction. This has 


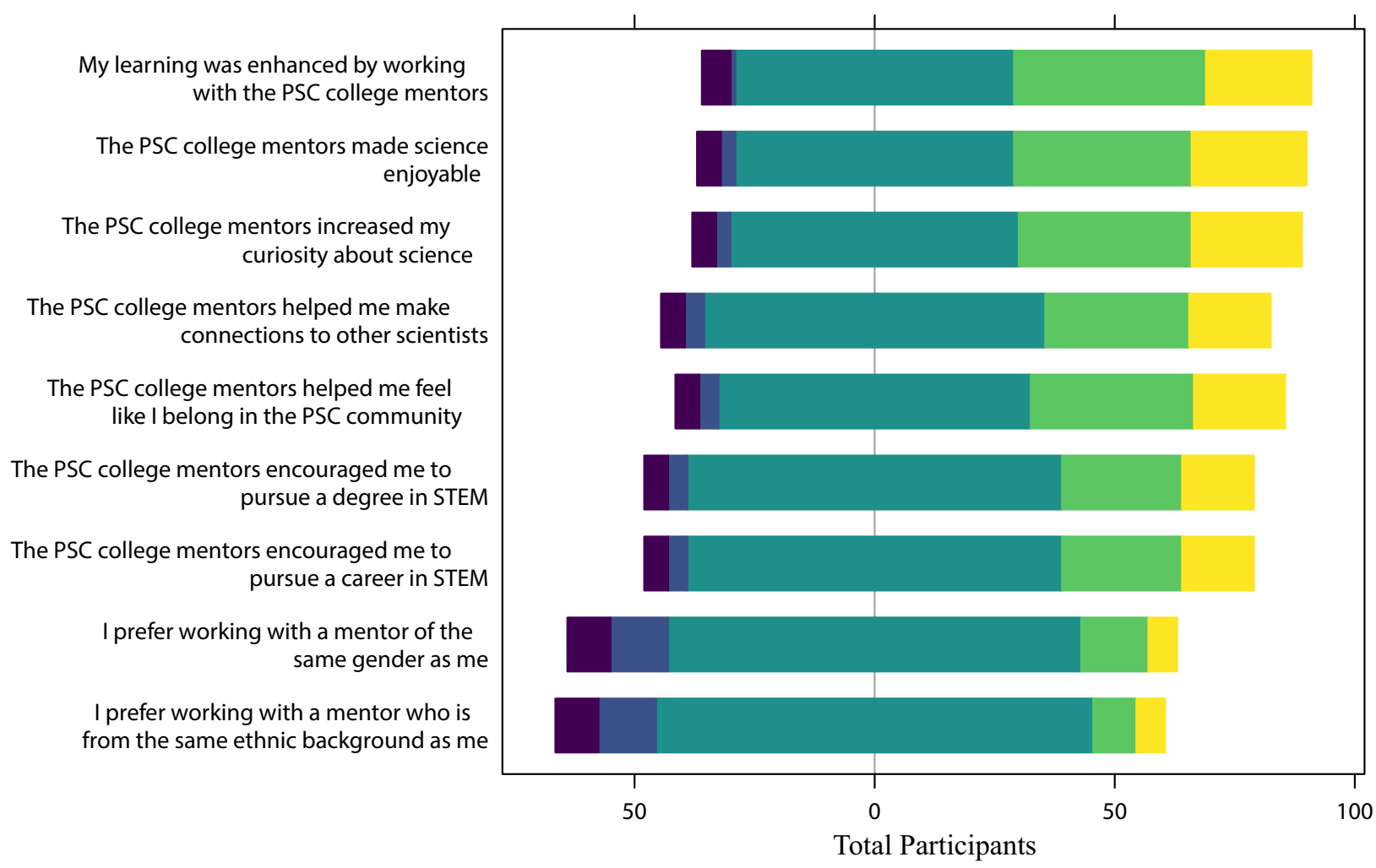

Strongly Disagree $\square \quad$ Disagree $\square \quad$ No Opinion $\square \quad$ Agree $\square$ Strongly Agree

Figure 8.2: PSC Post Survey Programmatic Responses Part 2

been flagged as an area for improvement in the future.

If the individuals answering "No Opinion" are ignored, then it does appear that the interactions that do occur are more positive than negative with $89.8 \%$ of respondents agreeing that working with the college mentors enhanced their learning and $81.6 \%$ indicating that working with the college mentors encouraged them to pursue both a STEM degree and STEM career. At most, only $7 \%$ of the total participants rated their experience with the college mentors as negative and, in each case, at least $31 \%$ of total respondents rated their experience as positive. Therefore, if the frequency of interactions between mentors and participants can be improved, those interactions would likely be positive and improve student enjoyment in the PSC and excitement about pursuing STEM in the future. 


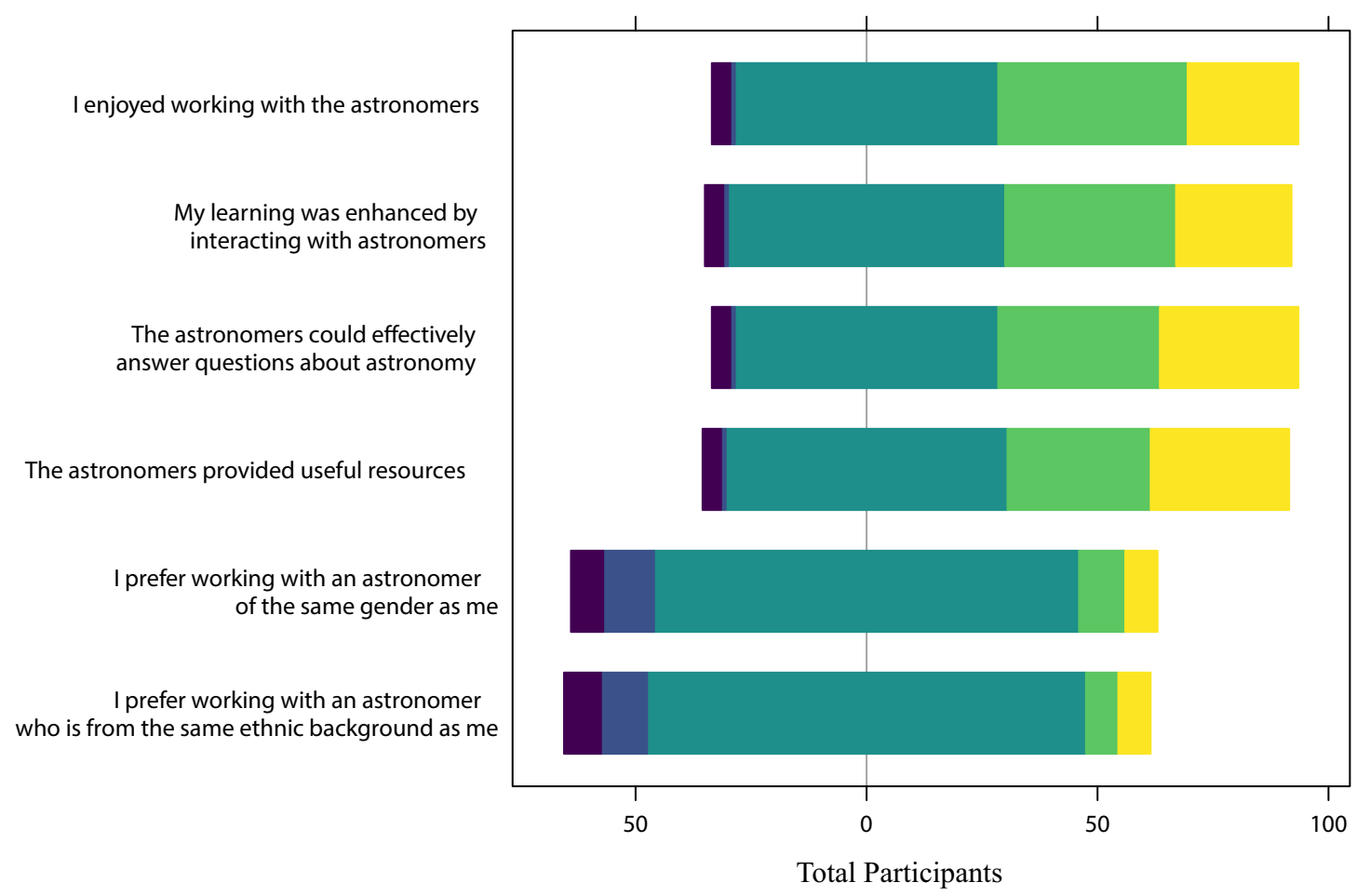

Strongly Disagree

Disagree

No Opinion

Agree

Strongly Agree

Figure 8.3: PSC Post Survey Programmatic Responses Part 3

The final question block in Fig. 8.3 repeats many of the questions of the last block, but with regard to the interactions with astronomers this time. Unlike with the mentors, a lower proportion of students respond with "No Opinion." This is most likely due to the online training and discussions sessions where students can easily interact with astronomers assisting with the PSC at regular times throughout the year. Those who do report opinions on these interactions indicate them to be overwhelmingly positive. Most students report "No Opinion" regarding gender or ethnic preference for the astronomers or mentors with whom they work. 


\subsection{Student Analysis}

For students participating in the PSC, pre-surveys were distributed through the PSC online portal before completing any training and post-surveys at the end of the academic year. Surveys from a control sample of students, students in the same science classes at the same schools as PSC participants but not in the PSC, were also collected. For those students participating in the control sample, surveys were collected in their science class and sent to the PSC research team for analysis on a similar time scale to that of the PSC participants. All items were scored on a 5-point scale.

There were 263 complete records for the pre-survey for PSC participants and 149 in the control sample. For the post-survey, there were 127 PSC participants and 149 control survey participants. There were 126 matched pairs on the pre- and post-surveys for the PSC participants and 149 matched pairs in the control sample.

\subsubsection{Pre-Survey Results}

Table 8.1 presents a comparison of subscale means between PSC participants and the control sample for the pre-survey. T-tests showed that there were significant differences between the groups in the domains of Self-Efficacy, Science Identity, and STEM Intentions. This result was expected; students choosing to participate in a STEM-focused activity should be more likely to already have a greater affinity for STEM above that of other high school students. Cohen's $d$ was used to characterize effect size. Cohen's convention indicates 0.2 as a small effect, 0.5 as a medium effect, and 0.8 as a large effect. The differences in Self-Efficacy, Science Identity, and STEM Intentions all represent very large effects. 


\begin{tabular}{|c|c|c|c|c|}
\hline & \multicolumn{2}{|c|}{ Mean \pm SD } & \multicolumn{2}{|l|}{} \\
\hline Domain & $\begin{array}{c}\text { PSC } \\
\text { Participants } \\
(\mathrm{N}=263)\end{array}$ & $\begin{array}{c}\text { Control } \\
\text { Participants } \\
(\mathrm{N}=149)\end{array}$ & $t$ & Cohen's $d$ \\
\hline Self-Efficacy & $76.4 \pm 17$ & $56.7 \pm 21$ & $9.99^{c}$ & 1.08 \\
\hline Science Identity & $67.4 \pm 12$ & $53.2 \pm 14$ & $10.23^{c}$ & 1.10 \\
\hline STEM Intentions & $77.5 \pm 20$ & $53.8 \pm 24$ & $10.08^{c}$ & 1.09 \\
\hline
\end{tabular}

Table 8.1: Pre-Survey Results: "a" $p<0.05$, "b" $p<0.01$, "c" $p<0.001$

\begin{tabular}{|c|c|c|c|c|}
\hline & \multicolumn{2}{|c|}{ Mean \pm SD } & \multicolumn{2}{|l|}{} \\
\hline Domain & $\begin{array}{c}\text { PSC } \\
\text { Participants } \\
(\mathrm{N}=127)\end{array}$ & $\begin{array}{c}\text { Control } \\
\text { Participants } \\
(\mathrm{N}=149)\end{array}$ & $t$ & Cohen's $d$ \\
\hline Self-Efficacy & $74.1 \pm 22$ & $57.7 \pm 22$ & $6.21^{c}$ & 0.75 \\
\hline Science Identity & $72.8 \pm 22$ & $53.9 \pm 20$ & $7.43^{c}$ & 0.90 \\
\hline STEM Intentions & $71.5 \pm 25$ & $52.3 \pm 27$ & $6.21^{c}$ & 0.75 \\
\hline
\end{tabular}

Table 8.2: Post-Survey Results: "a" $p<0.05$, "b" $p<0.01$, "c" $p<0.001$

\subsubsection{Post-Survey Results}

Table 8.2 presents a comparison of post-survey results for PSC students and students in the control sample. The differences in the mean levels of Self-Efficacy, Science Identity, and STEM Intentions remain large on the post survey, though smaller than on the pre-survey.

\subsubsection{Matched Samples}

Table 8.3 presents matched pre-survey and post-survey results for PSC students and Table 8.4 similar data for the control group. The results show a general decline in both SelfEfficacy and STEM intentions for PSC students with an increase in Science Identity. The control group, by contrast, does not show a significant change across any of the domains.

We hypothesize that the decrease in Self-Efficacy and STEM Intentions was caused by multiple factors such as an inflated sense of both at the start of the PSC due to excitement over participation in the program. As time passes and the PSC becomes a normal part 


\begin{tabular}{|c|c|c|c|c|}
\hline & \multicolumn{3}{|c|}{ Mean \pm SD } & \\
\hline Domain & $\begin{array}{c}\text { Pre-Survey } \\
(\mathrm{N}=126)\end{array}$ & $\begin{array}{c}\text { Post Survey } \\
(\mathrm{N}=126)\end{array}$ & $t$ & Cohen's $d$ \\
\hline Self-Efficacy & $78.1 \pm 17$ & $74.0 \pm 22$ & $3.19^{b}$ & 0.21 \\
\hline Science Identity & $67.9 \pm 14$ & $72.7 \pm 22$ & $-3.23^{b}$ & 0.26 \\
\hline STEM Intentions & $77.9 \pm 23$ & $71.3 \pm 25$ & $4.33^{c}$ & 0.28 \\
\hline
\end{tabular}

Table 8.3: Matched PSC Participants Pre/Post Survey Results: "a" $p<0.05$, "b" $p<0.01$, "c" $p<0.001$

\begin{tabular}{|c|c|c|c|c|}
\hline & \multicolumn{2}{|c|}{ Mean \pm SD } & \multicolumn{2}{c|}{} \\
\hline Domain & $\begin{array}{c}\text { Pre-Survey } \\
(\mathrm{N}=149)\end{array}$ & $\begin{array}{c}\text { Post Survey } \\
(\mathrm{N}=149)\end{array}$ & $t$ & Cohen's $d$ \\
\hline Self-Efficacy & $56.7 \pm 21$ & $57.7 \pm 22$ & .44 & 0.05 \\
\hline Science Identity & $53.2 \pm 14$ & $53.9 \pm 20$ & .35 & 0.04 \\
\hline STEM Intentions & $53.8 \pm 24$ & $52.3 \pm 27$ & .53 & 0.06 \\
\hline
\end{tabular}

Table 8.4: Matched Control Participants Pre/Post Survey Results: "a" $p<0.05$, "b" $p<0.01$, "c" $p<0.001$

of the student's life and the difficulties associated with participation in a "real" science project becomes clear, a student gains a more realistic understanding of the challenges of the scientific endeavor. Even with these decreases, PSC students still have significantly higher Self-Efficacy and STEM Intentions than their control sample peers. All changes represent small effects.

\subsection{Conclusions}

Throughout this study we identified a number of correctable issues in the programmatics of the New PSC. The first and most directly associated with the large attrition seen in both the Original and New PSC was that related to the qualification test structure. Based on the analysis shown here and follow-up interviews with PSC participants, the qualification test structure has been completely redesigned into a longer series of progressively more difficult "certification tests" that each must be passed at the $80 \%$ level and provide instant feedback 
to participants when they score a plot incorrectly. The other issues discussed here related to the diminished club size and capstone qualification illustrate the difficulty inherent in a distributed online-focused educational program. Despite a generally well performing initial program, more work and time was required to address the transition including identifying weaknesses in the initial PSC before moving forward with the New PSC. To move the program forward successfully, the online experience and hub model all must be redesigned to work more efficiently and overcome the challenges associated with diminished face-to-face interaction.

We found that participants were overwhelmingly those already interested in STEM. This was not surprising as the PSC is a much more involved scientific experience than other similar programs and therefore presents a higher barrier to entry. Though originally envisioned as a way to bring new people into STEM, the data suggest that the PSC serves much better as a retention tool, providing these students with a valuable STEM opportunity that most would not have until college. Though participant Self-Efficacy and STEM Intentions do diminish significantly throughout the program, they remain much higher than those of their peers who did not participate in the PSC, while Science Identity increased. If the PSC is improved programmatically and additional research-oriented activities were added, further analysis may yield improved results.

Implementation of the PSC as on online focused experience since 2016 has provided some key insights into the difficulties associated with the deployment of online learning content. Despite a nationwide reach via the internet, we saw only about the same total number of schools participating. With the Original PSC, WVU Astronomy was very active in its recruitment and support efforts. The New PSC did not have the same level of engagement 
from the hub institutions and the recruitment of new PSC clubs was likely hindered by this. This illustrates that such a transition requires highly engaged partners to be successful. There should also be clearly defined responsibilities at each level of the PSC with hub instructors, college mentors, teachers, and participants having a well defined picture of what their responsibilities are. The new PSC also had substantially fewer students participating per school, reducing the social aspect of the program and preventing the program from reaching students unsure about STEM. Improvements to the hub model should help to remedy this by replicating the high interaction of the Original PSC with the school clubs.

The difficulties associated with the development and maintenance of the online portal proved more challenging than initially expected. With the level of use required by the participants and the ability to get rapid feedback from users, it is necessary to have enough support to make changes quickly to the website. One feature repeatedly requested but as yet unimplemented is chatrooms for teachers, mentors, and hub instructors to share their experiences and provide support. Though not discussed here, there also existed a number of programming bugs in the sub-tests in the New PSC, that were discovered as students reported them, due to a lack of adequate resources for development and testing. 


\title{
Chapter 9
}

\author{
Future Works
}


Although this work provided some ways forward in addressing the leaky STEM pipeline, much more work must be done to increase the number of students who successfully complete STEM degrees. Future research plans revolve around the investigation of further applications of machine learning to retention and the evaluation and development of new assessment tools that can be used to provide more granular pictures of student learning to instructors. Some potential future and ongoing projects for the research group are outlined below:

- Use MIRT to evaluate the differences between the FCI and FMCE to determine if these instruments measure the same facets of student mechanics knowledge. Though both of these instruments have been used to measure the single domain of "Newtonian force concept" the construction of these instruments is vastly different. We seek to answer using MIRT if these two instruments should be used interchangeably.

- Construct machine learning models of overall STEM retention and build global early warning models of student departure. By applying the process applied to grade prediction, we want to generate an early warning system for academic advisors and students. This will not only serve to provide insights into where students may be in danger, but also free up time normally used to manually evaluate student performance to focus on working with students to develop plans to remediate issues and aid in their successful completion of degree programs.

- Use MIRT to construct and validate a new series of conceptual instruments that provide a multi-faceted overview of conceptual learning to instructors. As shown in the FCI and CSEM analysis, current instruments do not provide a fine grained picture of student knowledge. Using MIRT and modern test constructions techniques, we want to develop 
tests that can provide information to instructors and researchers about what concepts or aspects of physical concepts students understand. These more precise measurements could open up new avenues of research evaluating how to best improve learning of various aspects of physics beyond a "Newtonian force concept." This project would include multiple geographically distributed institutions with varying populations to provide both high data collection rates and a diverse validation sample.

- Evaluate the effectiveness of the changes implemented to the programmatic structure of the PSC. This will include the evaluation of the effect of the new qualification tests on retention of PSC students. 


\title{
Chapter 10
}

\author{
Additional Work
}


The following is a brief overview of submitted or published research that was not included in this thesis in order to improve the coherence of the thesis.

\title{
THE RELATION OF RACE/ETHNICITY AND GENDER TO PHYSICS CONCEPTUAL INVENTORY PERFORMANCE
}

Submitted for Publication in Physical Review Physics Education

\author{
Cabot Zabriskie ${ }^{1}$, Geraldine Cochran ${ }^{2}$, Seth DeVore ${ }^{1}$, Rachel Henderson ${ }^{3}$, John Stewart ${ }^{1}$, Paul \\ Miller $^{1}$, Gay Stewart ${ }^{1}$, Lynnette Michaluk ${ }^{4}$
}

Differences in performance of men and women on physics conceptual evaluations have been extensively studied. Very little research has examined underrepresented groups other than women in physics classes. This study reports racial/ethnic and gender differences in performance on the Force Concept Inventory $(N=2446)$, the Force and Motion Conceptual Evaluation $(N=3203)$, and the Conceptual Survey of Electricity and Magnetism $\left(N_{1}=\right.$ 2610, $N_{2}=2281$ ) in 4 samples drawn from two universities. The undergraduate populations at both universities were predominately White (77\% and 79\%). All samples show the often reported gender difference with men scoring higher on the post-test than women. In all samples, significant differences in post-test performance were measured between different racial/ethnic groups with effect sizes ranging up to a large effect $(d=0.93)$. The differences in performance by gender were largely independent of the differences by race/ethnicity. Controlling for academic performance using course or test grades and academic preparation measured by ACT or SAT mathematics scores greatly reduced and in some cases eliminated racial and ethnic differences, but had very little effect on gender differences. No race/ethnicity- 
by-gender interaction was detected in any sample. This result suggests that the differences in conceptual physics performance by race/ethnicity are a result of the high school academic preparation of the student and that failure to mitigate these differences in preparation differentially negatively impacts underrepresented students.

${ }^{1}$ Department of Physics and Astronomy, West Virginia University, Morgantown WV, 26506

${ }^{2}$ Department of Physics and Astronomy, Rutgers, The State University of New Jersey, Piscataway, NJ, 08854

3 Department of Physics and Astronomy, Michigan State University, East Lansing, MI 48824

${ }^{4}$ West Virginia University Center for Excellence in STEM Education, Morgantown WV, 26506

\section{THE IMPORTANCE OF BELONGING AND SELF-EFFICACY IN ENGINEERING IDENTITY}

Published: AERA open (2018) Link: https://par.nsf.gov/servlets/purl/10058182

Cabot Zabriskie $^{1}$, Rachel Henderson ${ }^{2}$, John Stewart ${ }^{1}$

Retention of students in Science, Technology, Engineering, and Mathematics (STEM) disciplines is a significant concern in higher education. Identity has been identified as an important correlate of academic success that may be important in a robust model of STEM retention. The engineering identity of "early career" university engineering students and its relation to GPA, self-efficacy, and a sense of belonging was examined. Self-efficacy and belonging were demonstrated to be domain dependent. A sense of belonging was much more strongly related to identity than either GPA or self-efficacy. A strong sense 
of belonging, specifically in the domain of the department of their major, was critical to a strong engineering identity.

${ }^{1}$ Department of Physics and Astronomy, West Virginia University, Morgantown WV, 26506

${ }^{2}$ Department of Physics and Astronomy, Michigan State University, East Lansing, MI 48824

\title{
RURAL AND FIRST GENERATION PERFORMANCE DIFFERENCES ON THE FORCE AND MOTION CONCEPTUAL EVALUATION
}

\author{
Published: Physics Education Research Conference 2018 Link: \\ https: //www. compadre. org/per/items/detail. cfm? ID $=14795$ \\ Rachel Henderson ${ }^{2}$, Cabot Zabriskie $^{1}$, John Stewart ${ }^{1}$
}

Differences in student performance on physics conceptual inventories have been studied with respect to gender and race/ethnicity. The current study expands this literature by exploring differences between first generation college students and rural/non-rural students on the Force and Motion Conceptual Evaluation (FMCE) using a large sample ( $\mathrm{N}=3325)$. Hierarchical linear regression was used to explore the effects of gender, race/ethnicity, firstgeneration status, and rural status. Significant differences in FMCE post-test scores were found by gender (14\%), race/ethnicity (7\%), first generation status (4\%), and rural status (5\%). Prior preparation, measured by ACT/SAT math scores, explained much of the performances by race/ethnicity and first generation status, but did not explain the differences in post-test scores by gender or rural status. No significant interactions between the different demographic features were measured 
${ }^{1}$ Department of Physics and Astronomy, West Virginia University, Morgantown WV, 26506

${ }^{2}$ Department of Physics and Astronomy, Michigan State University, East Lansing, MI 48824 


\section{Bibliography}

[1] National Science Board. Revisiting the STEM workforce: A companion to science and engineering indicators 2014. National Science Foundation VA, 2015.

[2] President's Council of Advisors on Science and Technology. Report to the President. Engage to Excel: Producing One Million Additional College Graduates with Degrees in Science, Technology, Engineering, and Mathematics. Executive Office of the President, Washington, DC, 2012.

[3] J. L. Docktor and J. P. Mestre. Synthesis of discipline-based education research in physics. Phys. Rev. Phys. Educ. Res., 10(2):020119, 2014.

[4] H. Metcalf. Stuck in the pipeline: A critical review of STEM workforce literature. InterActions: UCLA J. Educ. Inform. Stud., 6(2), 2010.

[5] S.A. Allen-Ramdial and A.G. Campbell. Reimagining the pipeline: Advancing STEM diversity, persistence, and success. BioScience, 64(7):612-618, 2014.

[6] N.A. Fouad and M.C. Santana. SCCT and underrepresented populations in STEM fields: Moving the needle. J. Career Assessment, 25(1):24-39, 2017.

[7] J. Nussbaum and J.D. Novak. An assessment of children's concepts of the earth utilizing structured interviews. Sci. Educ., 60(4):535-550, 1976.

[8] L. Leboutet-Barrell. Concepts of mechanics among young people. Phys. Educ., 11(7):462, 1976.

[9] A.B. Champagne, L.E. Klopfer, and J.H. Anderson. Factors influencing the learning of classical mechanics. Am. J. Phys., 48(12):1074-1079, 1980.

[10] J. Clement. Students' preconceptions in introductory mechanics. Am. J. Phys., $50(1): 66-71,1982$.

[11] L.C. McDermott. Research on conceptual understanding in mechanics. Phys. Today, $37: 24-32,1984$.

[12] I.A. Halloun and D. Hestenes. The initial knowledge state of college physics students. Am. J. Phys., 53(11):1043-1055, 1985.

[13] D. Hestenes, M. Wells, and G. Swackhamer. Force Concept Inventory. Phys. Teach., 30:141-158, 1992. 
[14] R.R. Hake. Interactive-engagement versus traditional methods: A six-thousandstudent survey of mechanics test data for introductory physics courses. Am. J. Phys., 66:64-74, 1998.

[15] R.K. Thornton and D.R. Sokoloff. Assessing student learning of Newton's laws: The Force and Motion Conceptual Evaluation and the evaluation of active learning laboratory and lecture curricula. Am. J. Phys., 66(4):338-352, 1998.

[16] D.P. Maloney, T.L. O'Kuma, C. Hieggelke, and A. Van Huevelen. Surveying students' conceptual knowledge of electricity and magnetism. Am. J. Phys., 69(S1):S12-S23, 2001.

[17] L. Ding, R. Chabay, B. Sherwood, and R. Beichner. Evaluating an electricity and magnetism assessment tool: Brief Electricity and Magnetism Assessment. Phys. Rev. Phys. Educ. Res., 2:010105, Mar 2006.

[18] N. Lasry, S. Rosenfield, H. Dedic, A. Dahan, and O. Reshef. The puzzling reliability of the Force Concept Inventory. Am. J. Phys., 79(9):909-912, 2011.

[19] A. Traxler, R. Henderson, J. Stewart, G. Stewart, A. Papak, and R. Lindell. Gender fairness within the Force Concept Inventory. Phys. Rev. Phys. Educ. Res., 14:010103, Jan 2018.

[20] J. M. Bland and D. G. Altman. Statistics notes: Cronbach's alpha. Bmj, 314(7080):572, 1997.

[21] E. Mazur. Peer Instruction: A User's Manual. Prentice Hall, Upper Saddle River, NJ, 1997.

[22] Physport. https://www.physport.org. Accessed 8/8/2017.

[23] C. Hieggelke and T. O'Kuma. The impact of physics education research on the teaching of scientists and engineers at two-year colleges. In 1997 Physics Education Research Conference Proceedings, volume 399, pages 267-288, New York, 1997. AIP, AIP Publishing.

[24] S.J. Pollock. Comparing student learning with multiple research-based conceptual surveys: CSEM and BEMA. AIP Conf. Proc., 1064:171-174, 2008.

[25] R.J. Dufresne, W.J. Gerace, W.J. Leonard, J.P. Mestre, and L. Wenk. Classtalk: A classroom communication system for active learning. J. Comput. High. Educ., 7(2):347, 1996.

[26] N.W. Reay, L. Bao, P. Li, R. Warnakulasooriya, and G. Baugh. Toward the effective use of voting machines in physics lectures. Am. J. Phys., 73(6):554-558, 2005.

[27] L. Ding, N.W. Reay, A. Lee, and L. Bao. Are we asking the right questions? validating clicker question sequences by student interviews. Am. J. Phys., 77(7):643-650, 2009. 
[28] C.H. Crouch, J. Watkins, A.P. Fagen, and E. Mazur. Peer instruction: Engaging students one-on-one, all at once. Research-based reform of university physics, 1(1):4095, 2007.

[29] L.C. McDermott and P.S. Shaffer. the physics education group at the university of washington. Tutorials in Introductory Physics, 1, 2002.

[30] L.C. McDermott and P.S. Shaffer. Research as a guide for curriculum development: An example from introductory electricity. part i: Investigation of student understanding. Am. J Phys., 60(11):994-1003, 1992.

[31] P.S. Shaffer and L.C. McDermott. Research as a guide for curriculum development: An example from introductory electricity. part ii: Design of instructional strategies. Am. J Phys., 60(11):1003-1013, 1992.

[32] N.D. Finkelstein and S.J. Pollock. Replicating and understanding successful innovations: Implementing Tutorials in Introductory Physics. Phys. Rev. Phys. Educ. Res., 1(1):010101, 2005.

[33] A. Van Heuvelen. Learning to think like a physicist: A review of research-based instructional strategies. Am. J. Phys., 59(891), 1991.

[34] E. Etkina, S. Murthy, and X. Zou. Using introductory labs to engage students in experimental design. Am. J. Phys., 74(11):979-986, 2006.

[35] C. Wieman and N.G. Holmes. Measuring the impact of an instructional laboratory on the learning of introductory physics. Am. J. Phys., 83(11):972-978, 2015.

[36] N.G. Holmes, J. Olsen, J.L. Thomas, and C.E. Wieman. Value added or misattributed? a multi-institution study on the educational benefit of labs for reinforcing physics content. Phys. Rev. Phys. Educ. Res., 13(1):010129, 2017.

[37] R.J. Beichner, J.M. Saul, D.S. Abbott, J.J. Morse, D. Deardorff, R.J. Allain, S.W. Bonham, M.H. Dancy, and J.S. Risley. The student-centered activities for large enrollment undergraduate programs (scale-up) project. Research-based reform of university physics, 1(1):2-39, 2007.

[38] S. Freeman, S.L. Eddy, M. McDonough, M.K. Smith, N. Okoroafor, H. Jordt, and M.Pat. Wenderoth. Active learning increases student performance in science, engineering, and mathematics. P. Nat. Acad. Sci. USA, 111(23):8410-8415, 2014.

[39] C. Henderson, M. Dancy, and M. Niewiadomska-Bugaj. Use of research-based instructional strategies in introductory physics: Where do faculty leave the innovationdecision process? Phys. Rev. Phys. Educ. Res., 8(2):020104, 2012.

[40] F. J. Gravetter and L. B. Wallnau. Statistics for the behavioral sciences. 2002.

[41] J. Welkowitz, H. B. Cohen, and B. R. Lea. Introductory statistics for the behavioral sciences. John Wiley \& Sons, 2011. 
[42] R. Nuzzo. How scientists fool themselves-and how they can stop. Nature News, 526(7572):182, 2015.

[43] J. Cohen. Statistical Power Analysis for the Behavioral Sciences. Academic Press, New York, NY, 1977.

[44] L. Pace. The "New" Statistics: Resampling and Bootstrapping, pages 257-268. Apress, Berkeley, CA, 2012.

[45] J. Stewart, C. Zabriskie, S. DeVore, and G. Stewart. Multidimensional item response theory and the force concept inventory. Phys. Rev. Phys. Educ. Res., 14:010137, Jun 2018.

[46] D. Huffman and P. Heller. What does the Force Concept Inventory actually measure? Phys. Teach., 33:138, 1995.

[47] T.F. Scott, D. Schumayer, and A.R. Gray. Exploratory factor analysis of a Force Concept Inventory data set. Phys. Rev. Phys. Educ. Res., 8(2):020105, 2012.

[48] M.R. Semak, R.D. Dietz, R.H. Pearson, and C.W. Willis. Examining evolving performance on the Force Concept Inventory using factor analysis. Phys. Rev. Phys. Educ. Res., 13:010103, Jan 2017.

[49] E. Brewe, J. Bruun, and I.G. Bearden. Using module analysis for multiple choice responses: A new method applied to Force Concept Inventory data. Phys. Rev. Phys. Educ. Res., 12(2):020131, 2016.

[50] R.P. Springuel, M.C. Wittmann, and J.R. Thompson. Applying clustering to statistical analysis of student reasoning about two-dimensional kinematics. Phys. Ed. Res., Am. J. Phys., 3(2):020107, 2007.

[51] J. Stewart, M. Miller, C. Audo, and G. Stewart. Using cluster analysis to identify patterns in students' responses to contextually different conceptual problems. Phys. Rev. Phys. Educ. Res., 8:020112, Oct 2012.

[52] J. Wang and L. Bao. Analyzing Force Concept Inventory with Item Response Theory. Am. J. Phys., 78(10):1064-1070, 2010.

[53] M. Planinic, L. Ivanjek, and A. Susac. Rasch model based analysis of the Force Concept Inventory. Phys. Rev. Phys. Educ. Res., 6:010103, Mar 2010.

[54] S. Osborn Popp, D. Meltzer, and M.C. Megowan-Romanowicz. Is the Force Concept Inventory biased? Investigating differential item functioning on a test of conceptual learning in physics. In 2011 American Educational Research Association Conference, Washington, DC, 2011. American Education Research Association.

[55] G.A. Morris, L. Branum-Martin, N. Harshman, S.D. Baker, E. Mazur, S. Dutta, T. Mzoughi, and V. McCauley. Testing the test: Item response curves and test quality. Am. J. Phys., 74(5):449-453, 2006. 
[56] G.A. Morris, N. Harshman, L. Branum-Martin, E. Mazur, T. Mzoughi, and S.D. Baker. An item response curves analysis of the Force Concept Inventory. Am. J. Phys., 80(9):825-831, 2012.

[57] L. Bao and E.F. Redish. Model analysis: Representing and assessing the dynamics of student learning. Phys. Rev. Phys. Educ. Res., 2:010103, Feb 2006.

[58] C. Henderson. Common concerns about the Force Concept Inventory. Phys. Teach., 40(9):542-547, 2002.

[59] N. Jorion, B.D. Gane, K. James, L. Schroeder, L.V. DiBello, and J.W. Pellegrino. An analytic framework for evaluating the validity of concept inventory claims. J. Eng. Educ., 104(4):454-496, 2015.

[60] D. Hestenes and I. Halloun. Interpreting the Force Concept Inventory: A response to March 1995 critique by Huffman and Heller. Phys. Teach., 33(8):502-502, 1995.

[61] P. Heller and D. Huffman. Interpreting the Force Concept Inventory: A reply to Hestenes and Halloun. Phys. Teach., 33(8):503-503, 1995.

[62] S. Ramlo. Validity and reliability of the Force and Motion Conceptual Evaluation. Am. J. Phys., 76(9):882-886, 2008.

[63] J. Han, L. Bao, L. Chen, T. Cai, Y. Pi, S. Zhou, Y. Tu, and K. Koenig. Dividing the Force Concept Inventory into two equivalent half-length tests. Phys. Rev. Phys. Educ. Res., 11:010112, May 2015.

[64] T.F. Scott and D. Schumayer. Students' proficiency scores within multitrait item response theory. Phys. Rev. Phys. Educ. Res., 11:020134, Nov 2015.

[65] Y. Lee, D.J. Palazzo, R. Warnakulasooriya, and D.E. Pritchard. Measuring student learning with item response theory. Phys. Ed. Res., Am. J. Phys., 4(1):010102, 2008.

[66] G.S. Gliner. College students' organization of mathematics word problems in relation to success in problem solving. School Sci. Math., 89:392-404, 1989.

[67] G.S. Gliner. College students' organization of mathematics word problems in terms of mathematical structure vs. surface structure. School Sci. Math., 91:105-110, 1991.

[68] B.S. Eylon and F. Reif. Effects of knowledge organization on task performance. Cognition Instruct., 1(1):5-44, 1984.

[69] M.T.H. Chi, P.J. Feltovich, and R. Glaser. Categorization and representation of physics problems by experts and novices. Cognitive Sci., 5(2):121-152, 1981.

[70] A.H. Schoenfeld and D.J. Herrmann. Problem perception and knowledge structure in expert and novice mathematical problem solvers. J. Exp. Psychol. Learn., 8(5):484, 1982. 
[71] F. Reif and J.I. Heller. Knowledge structure and problem solving in physics. Educ. Psychol., 17(2):102-127, 1982.

[72] I.D. Beatty and W.J. Gerace. Probing physics students' conceptual knowledge structures through term association. Am. J. Phys., 70(7):750-758, 2002.

[73] G. A. Miller. The magical number seven, plus or minus two: Some limits on our capacity for processing information. Psychol. Rev., 63(2):81, 1956.

[74] G.J. Posner, K.A. Strike, P.W. Hewson, and W.A. Gertzog. Accommodation of a scientific conception: Toward a theory of conceptual change. Sci. Educ., 66(2):211227, 1982.

[75] E. Etkina, J. Mestre, and A. O'Donnell. The cognitive revolution in educational psychology. pages 119-164. Information Age Publishing, Greenwich, CT, 2005.

[76] National Research Council. How People Learn: Brain, Mind, Experience, and School: Expanded Edition. The National Academies Press, Washington, DC, 2000.

[77] M.T.H. Chi and J.D. Slotta. The ontological coherence of intuitive physics. Cognition Instruct., 10(2-3):249-260, 1993.

[78] M.T.H. Chi, J.D. Slotta, and N. De Leeuw. From things to processes: A theory of conceptual change for learning science concepts. Learn. Instruct., 4(1):27-43, 1994.

[79] J.D. Slotta, M.T.H Chi, and E. Joram. Assessing students' misclassifications of physics concepts: An ontological basis for conceptual change. Cognition Instruct., 13(3):373400, 1995.

[80] A.A. DiSessa. Toward an epistemology of physics. Cognition Instruct., 10(2-3):105225, 1993.

[81] A.A. Disessa and B.L. Sherin. What changes in conceptual change? Int. J. Sci. Educ., 20(10):1155-1191, 1998.

[82] D. Hammer. Misconceptions or p-prims: How may alternative perspectives of cognitive structure influence instructional perceptions and intentions. J. Learn. Sci., 5(2):97$127,1996$.

[83] A.A. diSessa, N.M. Gillespie, and J.B. Esterly. Coherence versus fragmentation in the development of the concept of force. Cognitive Sci., 28(6):843-900, 2004.

[84] R.J. Dufresne, W.J. Leonard, and W.J. Gerace. Making sense of students' answers to multiple-choice questions. Phys. Teach., 40(3):174-180, 2002.

[85] R.N. Steinberg and M.S. Sabella. Performance on multiple-choice diagnostics and complementary exam problems. Phys. Teach., 35(3):150-155, 1997.

[86] A. Newell and H.A. Simon. Human Problem Solving. Prentice-Hall, Englewood Cliffs, NJ, 1972. 
[87] S. Ohlsson. The problems with problem solving: Reflections on the rise, current status, and possible future of a cognitive research paradigm. J. Prob. Solving, 5(1):7, 2012.

[88] J. Larkin, J. McDermott, D.P. Simon, and H.A. Simon. Expert and novice performance in solving physics problems. Science, 208(4450):1335-1342, 1980.

[89] J.H. Larkin, J. McDermott, D.P. Simon, and H.A. Simon. Models of competence in solving physics problems. Cognitive Sci., 4(4):317-345, 1980.

[90] A. Madsen, S.B. McKagan, and E. Sayre. Gender gap on concept inventories in physics: What is consistent, what is inconsistent, and what factors influence the gap? Phys. Rev. Phys. Educ. Res., 9:020121, Nov 2013.

[91] R. Henderson, G. Stewart, J. Stewart, L. Michaluk, and A. Traxler. Exploring the gender gap in the Conceptual Survey of Electricity and Magnetism. Phys. Rev. Phys. Educ. Res., 13:020114, Sep 2017.

[92] A.L. Traxler, X.C. Cid, J. Blue, and R. Barthelemy. Enriching gender in physics education research: A binary past and a complex future. Phys. Rev. Phys. Educ. Res., 12:020114, Aug 2016.

[93] US News \& World Report: Education. US News and World Report, Washington, DC. https://premium.usnews.com/best-colleges. Accessed 4/30/2017.

[94] W.J. van der Linden. Unidimensional Logistic Response Models. In Handbook of Item Response Theory, volume 1, pages 13-30. CRC Press, Taylor \& Francis Group, New York, NY, 2016.

[95] L. Hu and P.M. Bentler. Cutoff criteria for fit indexes in covariance structure analysis: Conventional criteria versus new alternatives. Struct. Equ. Modeling, 6(1):1-55, 1999.

[96] K.P. Burnham and D.R. Anderson. Model Selection and Multimodel Inference: A Practical Information-theoretic Approach. Springer-Verlag, New York, NY, 2003.

[97] R. McElreath. Statistical Rethinking: A Baysian Course with Examples in $R$ and Stan. CRC Press, Taylor \& Francis Group, Boca Raton, FL, 2016.

[98] A.E. Raftery. Bayesian model selection in social research. Sociol. Methodol., 25:111163, 1995.

[99] R.B. Kline. Principles and practices of structural equation modeling, fourth edition. Guilford Publications, New York, NY, 2016.

[100] K. Schermelleh-Engel, H. Moosbrugger, and H. Müller. Evaluating the fit of structural equation models: Tests of significance and descriptive goodness-of-fit measures. Methods of Psychological Research Online, 8(2):23-74, 2003.

[101] R Core Team. R: A Language and Environment for Statistical Computing. R Foundation for Statistical Computing, Vienna, Austria, 2013. 
[102] R.P. Chalmers. mirt: A multidimensional item response theory package for the R environment. J. Stat. Soft., 48(6):1-29, 2012.

[103] S. Epskamp, A.O.J. Cramer, J.L. Waldorp, V.D. Schmittmann, and D. Borsboom. qgraph: Network visualizations of relationships in psychometric data. J. Stat. Soft., 48(4):1-18, 2012.

[104] A. Canty and B.D. Ripley. boot: Bootstrap R (S-Plus) Functions, 2017. R package version 1.3-20.

[105] A.C. Davison and D.V. Hinkley. Bootstrap Methods and Their Applications. Cambridge University Press, Cambridge, UK, 1997.

[106] L.J. Cronbach and P.E. Meehl. Construct validity in psychological tests. Psychol. Bull., 52(4):281, 1955.

[107] L.A. Clark and D. Watson. Constructing validity: Basic issues in objective scale development. Psychol. Assessment, 7(3):309, 1995.

[108] B.T West, K.B. Welch, and A.T. Gatecki. Linear Mixed Models: A Practical Guide to Using Statistical Software. CRC Press, Francis \& Taylor Group, Boca Raton, FL, 2nd edition, 2015.

[109] See Supplemental Material at [URL will be inserted by publisher] for traditional factor analysis, 3 and 5 factor MIRT factor models, and the constrained MIRT model with the factor loading on all items.

[110] L. Crocker and J. Algina. Introduction to Classical and Modern Test Theory. Holt, Rinehart and Winston, Mason, OH, 1986.

[111] M. Planinic. Assessment of difficulties of some conceptual areas from electricity and magnetism using the Conceptual Survey of Electricity and Magnetism. Am. J. Phys., 74(12):1143-1148, 2006.

[112] D.E. Meltzer. Analysis of shifts in students' reasoning regarding electric field and potential concepts. In L. McCullough, L. Hsu, and P. Heron, editors, 2006 Physics Education Research Conference Proceedings, volume 883, pages 177-180, New York, 2007. AIP, AIP Publishing.

[113] J. Leppävirta. The effect of naïve ideas on students' reasoning about electricity and magnetism. Res. Sci. Educ., 42(4):753-767, 2012.

[114] P.B. Kohl and H.V. Kuo. Introductory physics gender gaps: Pre-and post-studio transition. AIP Conf. Proc., 1179:173-176, 2009.

[115] J.M. Wilson. The CUPLE physics studio. Phys. Teach., 32(9):518-523, 1994.

[116] K. Kreutzer and A. Boudreaux. Preliminary investigation of instructor effects on gender gap in introductory physics. Phys. Rev. Phys. Educ. Res., 8(1):010120, 2012. 
[117] The Carnegie Classification of Institutions of Higher Education. Center for Postsecondary Research, Indiana University School of Education, Bloomington, IN. http://carnegieclassifications.iu.edu/. Accessed 9/21/2017.

[118] R. Henderson, P. Miller, J. Stewart, A. Traxler, and R. Lindell. Item-level gender fairness in the Force and Motion Conceptual Evaluation and the Conceptual Survey of Electricity and Magnetism. arXiv preprint arXiv:1803.02399, 2018.

[119] V. Otero, S. Pollock, and N. Finkelstein. A physics department's role in preparing physics teachers: The Colorado Learning Assistant model. Am. J. Phys., 78(11):1218$1224,2010$.

[120] L.C. McDermott and P.S. Shaffer. Tutorials in Introductory Physics. Prentice Hall, Upper Saddle River, NJ, 1998.

[121] X. Chen. Stem attrition: College students' paths into and out of stem fields. statistical analysis report. nces 2014-001. National Center for Education Statistics, 2013.

[122] K. Rask. Attrition in stem fields at a liberal arts college: The importance of grades and pre-collegiate preferences. Econ Educ Rev, 29(6):892-900, 2010.

[123] E. J. Shaw and S. Barbuti. Patterns of persistence in intended college major with a focus on stem majors. NACADA Journal, 30(2):19-34, 2010.

[124] A. V. Maltese and R. H. Tai. Pipeline persistence: Examining the association of educational experiences with earned degrees in stem among us students. Sci Educ, 95(5):877-907, 2011.

[125] G. Zhang, T. J. Anderson, M. W. Ohland, and B. R. Thorndyke. Identifying factors influencing engineering student graduation: A longitudinal and cross-institutional study. J Eng Educ, 93(4):313-320, 2004.

[126] B. F. French, J. C. Immekus, and W. C. Oakes. An examination of indicators of engineering students' success and persistence. J Eng Educ, 94(4):419-425, 2005.

[127] R. M. Marra, K. A. Rodgers, D. Shen, and B. Bogue. Leaving engineering: A multiyear single institution study. J Eng Educ, 101(1):6-27, 2012.

[128] C. W. Hall, P. J. Kauffmann, K. L. Wuensch, W. E. Swart, K. A. DeUrquidi, H. O. Griffin, and S. C. Duncan. Aptitude and personality traits in retention of engineering students. J Eng Educ, 104(2):167-188, 2015.

[129] P. Baepler and C. J. Murdoch. Academic analytics and data mining in higher education. International Journal for the Scholarship of Teaching and Learning, 4(2):17, 2010.

[130] R. S. J. D. Baker and K. Yacef. The state of educational data mining in 2009: A review and future visions. J Educ Data Mining, 1(1):3-17, 2009. 
[131] Z. Papamitsiou and A. A. Economides. Learning analytics and educational data mining in practice: A systematic literature review of empirical evidence. J Educ Tech and Society, 17(4), 2014.

[132] A. Dutt, M. A. Ismail, and T. Herawan. A systematic review on educational data mining. IEEE Access, 5:15991-16005, 2017.

[133] C. Romero and S. Ventura. Educational data mining: a review of the state of the art. IEEE Transactions on Systems, Man, and Cybernetics, Part C (Applications and Reviews), 40(6):601-618, 2010.

[134] J.M. Aiken, R. Henderson, and M.D. Caballero. Modeling student pathways in a physics bachelor's degree program. arXiv preprint arXiv:1810.11272, 2018.

[135] E. Brewe, V. Sawtelle, L.H. Kramer, G.E. O'Brien, I. Rodriguez, and P. Pamelá. Toward equity through participation in Modeling Instruction in introductory university physics. Phys. Rev. ST Phys. Educ. Res., 6(1):010106, 2010.

[136] C.H. Crouch and E. Mazur. Peer instruction: Ten years of experience and results. Am. J Phys, 69(9):970-977, 2001.

[137] K. Cummings, J. Marx, R. Thornton, and D. Kuhl. Evaluating innovation in studio physics. Am. J Phys, 67(S1):S38-S44, 1999.

[138] B. Thacker, H. Dulli, D. Pattillo, and K. West. Lessons from a large-scale assessment: Results from conceptual inventories. Phys. Rev. Phys. Educ. Res., 10(2):020104, 2014.

[139] C. Romero and S. Ventura. Educational data mining: A survey from 1995 to 2005. Expert Syst. Appl., 33(1):135-146, 2007.

[140] A. Peña-Ayala. Educational data mining: A survey and a data mining-based analysis of recent works. Expert Syst. Appl., 41(4):1432-1462, 2014.

[141] U. bin Mat, N. Buniyamin, P.M. Arsad, and R. Kassim. An overview of using academic analytics to predict and improve students' achievement: A proposed proactive intelligent intervention. In Engineering Education (ICEED), 2013 IEEE 5th Conference on, pages 126-130. IEEE, 2013.

[142] C. Romero, S. Ventura, P.G. Espejo, and C. Hervás. Data mining algorithms to classify students. In R.S. Joazeiro de Baker, T. Barnes, and J.E. Beck, editors, Proceeding of the 1st International Conference on Educational Data Mining, Montreal, Quebec, Canada, 2008.

[143] A.M. Shahiri, W. Husain, and N.A. Rashid. A review on predicting student's performance using data mining techniques. Procedia Comput. Sci., 72:414-422, 2015.

[144] G. James, D. Witten, T. Hastie, and R. Tibshirani. An Introduction to Statistical Learning with Applications in R, volume 112. Springer-Verlag, New York, NY, 2017. 
[145] A.C. Müller and S. Guido. Introduction to Machine Learning with Python: A Guide for Data Scientists. O'Reilly Media, Boston, MA, 2016.

[146] S. Huang and N. Fang. Predicting student academic performance in an engineering dynamics course: A comparison of four types of predictive mathematical models. Comput. Educ., 61:133-145, 2013.

[147] F. Marbouti, H.A. Diefes-Dux, and K. Madhavan. Models for early prediction of at-risk students in a course using standards-based grading. Comput. Educ., 103:1-15, 2016.

[148] L.P. Macfadyen and S. Dawson. Mining LMS data to develop an "early warning system" for educators: A proof of concept. Comput. Educ., 54(2):588-599, 2010.

[149] Y. Hu, C. Lo, and S. Shih. Developing early warning systems to predict students' online learning performance. Comput. Hum. Behav., 36:469-478, 2014.

[150] C.L. Ballard and M.F. Johnson. Basic math skills and performance in an introductory economics class. J. Econ. Educ., 35(1):3-23, 2004.

[151] M. Abdous, H. Wu, and C. Yen. Using data mining for predicting relationships between online question theme and final grade. J. Educ. Tech. Soc., 15(3):77, 2012.

[152] A. Wojciechowski and L.B. Palmer. Individual student characteristics: Can any be predictors of success in online classes. Online J. Distance Learn. Adm., 8(2):13, 2005.

[153] R.K. Thornton and D.R. Sokoloff. Assessing student learning of Newton's laws: The Force and Motion Conceptual Evaluation and the evaluation of active learning laboratory and lecture curricula. Am. J. Phys., 66(4):338-352, 1998.

[154] D. Conway and J. White. Machine Learning for Hackers. O'Reilly Media, Boston, MA, 2012.

[155] T. Fawcett. An introduction to ROC analysis. Pattern Recogn. Lett., 27(8):861-874, 2006.

[156] D.G. Altman. Practical Statistics for Medical Research. CRC Press, Boca Raton, FL, 1990 .

[157] D.W. Hosmer Jr, S. Lemeshow, and R.X. Sturdivant. Applied Logistic Regression, volume 398. John Wiley \& Sons, New York, NY, 2013.

[158] W.N. Venables and B.D. Ripley. Modern Applied Statistics with S. Springer-Verlag, New York, NY, fourth edition, 2002.

[159] L. Breiman, J.H. Friedman, R.A. Olshen, and C.J. Stone. Classification and Regression Trees. Wadsworth \& Brooks/Cole, Monterey, CA, 1984.

[160] T. Hastie, R. Tibshirani, and J. Friedman. The Elements of Statistical Learning: Data Mining, Inference, and Prediction. Springer-Verlag, New York, NY, 2009. 
[161] A. Liaw and M. Wiener. Classification and regression by randomForest. $R$ News, $2(3): 18-22,2002$.

[162] M.T. Ribeiro, S. Singh, and C. Guestrin. Why should I trust you?: Explaining the predictions of any classifier. In Proceedings of the 22nd ACM SIGKDD International Conference on Knowledge Discovery and Data Mining, pages 1135-1144. ACM, 2016.

[163] R Henderson and J Stewart. Racial and ethnic bias in the Force Concept Inventory. 2017 Phys. Educ. Res. Conf. Proceedings, 2017 in press.

[164] R. Henderson, C. Zabriskie, and J. Stewart. Rural and first generation performance differences on the Force and Motion Conceptual Evaluation. Physics Education Research Conference Proceedings 2018 (accepted)., 2018.

[165] D. Voyer and S.D. Voyer. Gender differences in scholastic achievement: A metaanalysis. Psychol. Bull., 140(4):1174, 2014.

[166] K. Williamson, M. McLaughlin, S. A. Heatherly, J. Stewart, D. Lorimer, H. Blumer, C. Zabriskie, and R. Lynch. The pulsar search collaboratory: Expanding nationwide. arXiv preprint arXiv:180\%.06059, 2018.

[167] R. Rosen, J. Swiggum, M. A. McLaughlin, D. R. Lorimer, M. Yun, S. A. Heatherly, J. Boyles, R. Lynch, V. I. Kondratiev, S. Scoles, et al. The pulsar search collaboratory: discovery and timing of five new pulsars. The Astrophysical Journal, 768(1):85, 2013.

[168] R. Rosen, S. A. Heatherly, M. A. McLaughlin, R. Lynch, V. I. Kondratiev, J. R. Boyles, M. T. Wilson, D. R. Lorimer, and S. Ransom. The pulsar search collaboratory. arXiv preprint arXiv:1005.1060, 2010.

[169] Zooniverse. Available at https://www.zooniverse.org/.

[170] Z. Hazari, G. Sonnert, P.M. Sadler, and M. Shanahan. Connecting high school physics experiences, outcome expectations, physics identity, and physics career choice: A gender study. J Res. Sci. Teach., 47(8):978-1003, 2010.

[171] T. Perez, J.G. Cromley, and A. Kaplan. The role of identity development, values, and costs in college stem retention. J. Educ. Psychol., 106(1):315, 2014.

[172] P. R. Aschbacher, E. Li, and E. J. Roth. Is science me? High school students' identities, participation and aspirations in science, engineering, and medicine. J. Res. Sci. Teach., 47(5):564-582, 2010.

[173] S.M. Glynn, P. Brickman, N. Armstrong, and G. Taasoobshirazi. Science motivation questionnaire ii: Validation with science majors and nonscience majors. J. Res. Sci. Teach., 48(10):1159-1176, 2011.

[174] G. Trujillo and K.D. Tanner. Considering the role of affect in learning: Monitoring students' self-efficacy, sense of belonging, and science identity. CBE-Life Sci. Educ., 13(1):6-15, 2014. 
[175] D. Anderson-Butcher and D.E. Conroy. Factorial and criterion validity of scores of a measure of belonging in youth development programs. Educ. Psychol. Meas., 62(5):857-876, 2002.

[176] M.W. Kier, M.R. Blanchard, J.W. Osborne, and J.L. Albert. The development of the stem career interest survey (stem-cis). R. Sci. Educ., 44(3):461-481, 2014.

[177] T. Tyler-Wood, G. Knezek, and R. Christensen. Instruments for assessing interest in stem content and careers. J. Tech. Teach. Educ., 18(2):345-368, 2010. 